Desenvolvimento de esquema upwind para equações de conservação e implementação de modelagens URANS com aplicação em escoamentos incompressíveis

Miguel Antonio Caro Candezano 


\title{
Desenvolvimento de esquema upwind para equações de conservação e implementação de modelagens URANS com aplicação em escoamentos incompressíveis
}

\author{
Miguel Antonio Caro Candezano
}

Orientador: Prof. Dr. Valdemir Garcia Ferreira

Tese apresentada ao Instituto de Ciências Matemáticas e de Computação - ICMC-USP, como parte dos requisitos para obtenção do título de Doutor em

Ciências - Ciências de Computação e Matemática Computacional. VERSÃO REVISADA

USP - São Carlos

Dezembro de 2012 
Ficha catalográfica elaborada pela Biblioteca Prof. Achille Bassi e Seção Técnica de Informática, ICMC/USP, com os dados fornecidos pelo(a) autor(a)

Caro Candezano, Miguel Antonio

Desenvolvimento de esquema upwind para equações de conservação e implementação de modelagens URANS com aplicação em escoamentos incompressíveis / Miguel Antonio Caro Candezano; orientador Valdemir Garcia Ferreira. -- São Carlos, 2012.

$171 \mathrm{p}$.

Tese (Doutorado - Programa de Pós-Graduação em Ciências de Computação e Matemática Computacional) -Instituto de Ciências Matemáticas e de Computação, Universidade de São Paulo, 2012.

1. termos convectivos. 2. leis de conservação. 3. equações de Navier-Stokes. 4. escoamentos com superfícies livres móveis. 5. modelagem k-e, RNG k-e. I. Garcia Ferreira, Valdemir, orient. II. Título. 
Dedico esta tese a minha esposa María e a meus filhos Mateo e Alejandro, pelos quase quatro anos em que tive de me fazer ausente de suas vidas nos momentos que mais me precisavam. A vocês, minhas desculpas e a minha eterna gratidão. 



\section{Agradecimentos}

Ao meu amigo e orientador Prof. Dr. Valdemir Garcia Ferreira pela disponibilidade, apoio, confiança, seus ensinamentos e orientações, e principalmente pela paciência.

Aos meus pais, Luz María e Miguel Antonio pelo grande amor, paciência, compreensão e grande apoio ao longo de todos os estudos. Também, aos meus irmãos J. Carlos, Sabas, Rebeca, Gladys, Luz Mary, Patricia, Julio e Luisa.

Ao meus amigos Alysson N. Silva, Alexandre De Lacassa e Rafael A. Figueiredo pelos conselhos, pela ajuda e pela companhia nos momentos difíceis.

Ao meu amigo Everton Alvares Cherman, por toda ajuda nos momentos mais difíceis da minha estadia no Brasil.

Aos meus amigos Lais, Patrícia, Josuel, Giseli, Larisa, Douglas, Juliana, Italo e Gabriela por toda ajuda e compreensão.

Aos meus amigos Alfredo, Henry, John, Ingrid, Edwin, Jeinny, Rodolfo e Miguel por sua amizade e companhia.

Aos funcionários do ICMC-USP por toda dedicação, em especial ao Leonardo Martinussi por toda sua paciência e ajuda.

A todos os professores do LMACC-ICMC/USP, em especial ao Prof. Dr. Gustavo Carlos Buscaglia pelos ensinamentos e reflexões.

A CAPES com o programa PEC-PG, pelo suporte financeiro concedido para realização do meu projeto de pesquisa.

Finalmente, agradeço a todos que direta ou indiretamente contribuíram para a realização deste trabalho.

A Deus!. 


\section{Resumo}

Nesta tese é apresentado um esquema novo de alta resolução upwind (denominado TDPUS-C3) para reconstrução de fluxos numéricos para leis de conservação não lineares e problemas relacionados em DFC. O esquema é baseado nos critérios de estabilidade CBC e TVD e desenvolvido utilizando condições de diferenciabilidade $C^{3}$. Além disso, é realizada a implementação da associação do esquema TDPUS-C3 com a modelagem de turbulência $R N G \kappa-\varepsilon$. O propósito é obter soluções numéricas de sistemas hiperbólicos de leis de conservação para dinâmica dos gases e equações de Navier-Stokes para escoamento incompressível de fluidos newtonianos e não newtonianos (viscoelásticos). Fazendo o uso do esquema TDPUS-C3, a precisão global dos métodos numéricos é verificada acessando o erro em problemas teste (benchmark) 1D e 2D. Um estudo comparativo entre os resultados do esquema TDPUS-C3 e os de esquemas upwind convencionais para leis de conservação hiperbólicas complexas é também realizado. A associação das modelagens numéricas (upwinding mais $R N G \kappa-\varepsilon$ ) é, então, examinada na simulação de escoamentos turbulentos de fluidos newtonianos envolvendo superfícies livres móveis, usando a metodologia URANS. No geral, em termos do comportamento global, concordância satisfatória é observada.

Palavras-chave:: termos convectivos, leis de conservação, captura de choque, equações de Navier-Stokes, escoamentos com superfícies livres móveis, modelagem $\kappa-\varepsilon$ e $R N G \kappa-\varepsilon$. 


\section{Abstract}

In this thesis, a new high-resolution upwind scheme (named TDPUS-C3) for reconstruction of numerical fluxes for nonlinear conservation laws and related CFD problems is presented. The scheme is based on CBC and TVD stability criteria and developed by employing differentiability conditions $\left(C^{3}\right)$. In addition, the implementation of an association of the TDPUS-C3 scheme with the $R N G \kappa-\varepsilon$ turbulence modelling is also performed. The purpose is to obtain numerical solutions of systems of hyperbolic conservation laws for gas dynamics and Navier-Stokes equations for incompressible flow of Newtonian and non-Newtonian (viscoelastic) fluids. By using the TDPUS-C3 scheme, the global accuracy of the numerical methods is verified by assessing the error on $1 \mathrm{D}$ and $2 \mathrm{D}$ benchmark test cases. A comparative study between the TDPUS-C3 scheme and conventional upwind schemes to solve standard and complex hyperbolic conservation laws is also accomplished. The association of the numerical modelling (upwinding plus $R N G \kappa-\varepsilon$ ) is then examined in the simulation of turbulent Newtonian fluid flows involving moving free surfaces, by using URANS methodology. Overall, satisfactory agreement is found in terms of the overall behaviour.

Key words: convective terms, conservation laws, shock capturing, Navier-Stokes equations, moving free surface flows, $\kappa-\varepsilon$ turbulence modelling, $R N G \kappa-\varepsilon$. 


\section{Sumário}

1 Introdução $\quad 1$

1.1 Tema da pesquisa . . . . . . . . . . . . . . . . . . . . . . . . 1

1.2 Desenvolvimento de modelagens numéricas . . . . . . . . . . . . . . 1

1.3 Motivação, objetivo e contribuição do presente estudo . . . . . . . . . . . . . 3

1.4 Estrutura da tese e equipamentos utilizados . . . . . . . . . . . . . . . . 4

2 Esquemas convectivos upwind $\quad \mathbf{7}$

2.1 Motivação e aproximação upwind . . . . . . . . . . . . . . . . . . . 7

2.1 .1 Critérios de limitação . . . . . . . . . . . . . . . . . . . . . . . . . . . . . . .

2.1 .2 Limitadores de fluxo . . . . . . . . . . . . . . . . . . . . 15

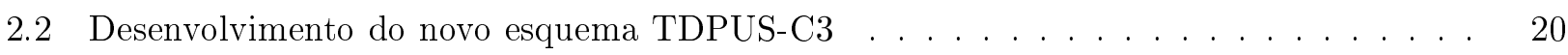

3 Formulação de sistemas hiperbólicos e metodologia de solução 25

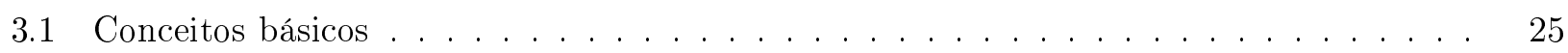

3.2 Métodos tipo Godunov e algoritmos de propagação de ondas . . . . . . . . . . . . . 29

3.3 Discretização das leis de conservação . . . . . . . . . . . . . . . . . . . . . . 32

3.3 .1 Advecção de escalares . . . . . . . . . . . . . . . . . . . . . . . . . . . . . . . . . . . . . . . . . . . . . . . .

3.3 .2 Equação de Burgers . . . . . . . . . . . . . . . . . . . . . . . 33

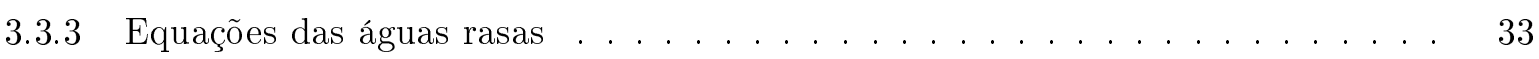

3.4 A marcha no tempo . . . . . . . . . . . . . . . . . . . . . . 33

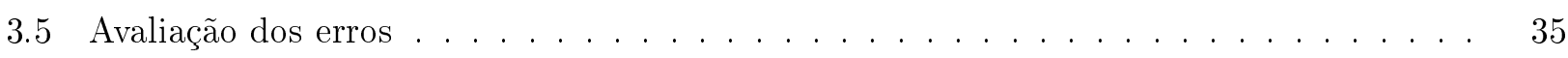

4 Formulação de escoamentos incompressíveis, metodologia de solução e equações $\begin{array}{ll}\text { discretas } & \mathbf{3 7}\end{array}$

4.1 Equações básicas . . . . . . . . . . . . . . . . . . . . . . . 37

4.1.1 Formulação matemática para fluidos newtonianos . . . . . . . . . . . . . 39

4.1.2 Modelagem matemática para fluidos newtonianos em regime turbulento . . . . 39

4.1 .3 Formulação matemática para fluidos não newtonianos . . . . . . . . . . . . . . 43

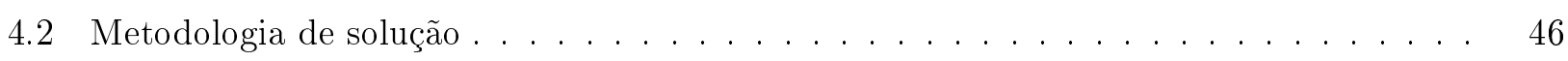

4.2 .1 Método de projeção e algoritmo computacional . . . . . . . . . . . . . . 47 


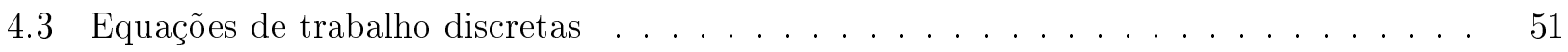

4.4 Condições adicionais adotadas e suas discretizações . . . . . . . . . . . . . . . 58

4.4 .1 Condições iniciais . . . . . . . . . . . . . . . . . . . . . . 58

4.4 .2 Condições de contorno . . . . . . . . . . . . . . . . . . . . 59

5 Resultados numéricos para sistemas hiperbólicos 1D e 2D $\quad 67$

5.1 Escolha do parâmetro livre $\beta$ para o esquema TDPUS-C3 . . . . . . . . . . . 67

5.2 Equações 1D e 2D de leis conservação . . . . . . . . . . . . . . . . . . . 69

5.2 .1 Advecção de escalares . . . . . . . . . . . . . . . . . . . . 69

5.2 .2 Equação não linear de Burgers . . . . . . . . . . . . . . . . . . . . 72

5.2 .3 Equação não linear de Buckley-Leverett . . . . . . . . . . . . . . . . . . . 77

5.3 Sistemas 1D e 2D de leis de conservação . . . . . . . . . . . . . . . . . . . 79

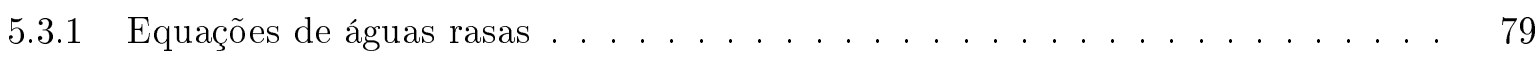

5.3 .2 Equações de Euler . . . . . . . . . . . . . . . . . . 85

5.3 .3 Equações da magnetohidrodinâmica . . . . . . . . . . . . . . . 102

6 Simulação de escoamentos incompressíveis $\quad 105$

6.1 Resultados para fluidos newtonianos . . . . . . . . . . . . . . . . 105

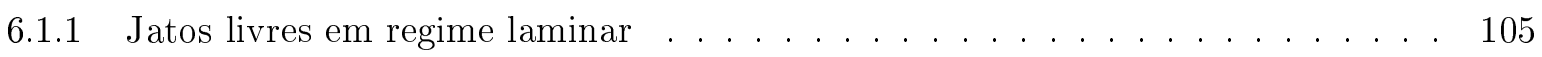

6.1.2 Colapso de um bloco de fluido em regime laminar . . . . . . . . . . . . . . 111

6.1.3 Jatos livres 3D a baixos valores do número de Reynolds . . . . . . . . . . . . 114

6.1.4 Jatos livres 2D e 3D no regime turbulento . . . . . . . . . . . . . . . 124

6.1.5 Colapso de fluido e interação da superfície livre com um obstáculo . . . . . . . 134

6.2 Resultados para fluidos não newtonianos 3D . . . . . . . . . . . . . 137

7 Considerações finais e trabalhos futuros $\quad 141$

$\begin{array}{ll}\text { A Produção científica associada } & 143\end{array}$

B Código para solução da equação de Burgers 2D utilizando a linguagem C $++\quad 151$

$\begin{array}{lr}\text { Referências bibliográficas } & 160\end{array}$ 


\section{Lista de Figuras}

2.1 Representação esquemática do campo de velocidades de um escoamento de fluido. . . . 7

2.2 Solução analítica do problema de advecção com velocidade de convecção $u>0$. . . . . 8

2.3 Molécula computacional da discretização dos termos convectivos no ponto $\mathcal{P}$. . . . . . 9

2.4 Região de monotonicidade para esquemas de segunda ordem no diagrama de variáveis normalizadas. . . . . . . . . . . . . . . . . . . . . . . . . . . 10

2.5 Diagrama de variáveis normalizadas: Região CBC. . . . . . . . . . . . . . . . . . . . 11

2.6 Diagrama de variáveis normalizadas: Região ECBC. . . . . . . . . . . . . . . . . . . 12

2.7 DVN: Região TVD. . . . . . . . . . . . . . . . . . . . . . . . . . 13

2.8 Comparação entra as soluções numéricas obtidas com o esquema Lax-Wendroff e analítica do problema de advecção. $N=2000$ e $C F L=0.3$. . . . . . . . . . . . . . . . . . . 16

2.9 Plano $\Psi\left(r_{f}\right) \perp r_{f}$ mostrando a região de extremos $\left(r_{f} \leq 0\right)$, a região de monotonicidade (vizinhança de $r_{f}=1$ ) e as regiões de alta curvatura $\left(r_{f}>>1\right.$ e $r_{f}<<1$ ). . . . . . 17

2.10 Região TVD de Sweby. . . . . . . . . . . . . . . . . . . . . . . 18

2.11 Região TVD de segunda ordem nas variáveis $(\Psi, r)$. . . . . . . . . . . . . . . . . . . . 19

2.12 Região TVD para o esquema TDPUS-C3 em variáveis normalizadas. . . . . . . . . . 22

2.13 Região TVD para o esquema TDPUS-C3 em termos de limitadores de fluxo. . . . . . 23

3.1 Região de integração para o cálculo da velocidade do choque. . . . . . . . . . . . . . 27

3.2 Onda de choque. . . . . . . . . . . . . . . . . . . . . . . . . . 28

3.3 Onda de rarefação. . . . . . . . . . . . . . . . . . . . . . . . . . . . . . . . 28

3.4 Solução típica para um problema de Riemann das equações de Euler. . . . . . . . . . . 28

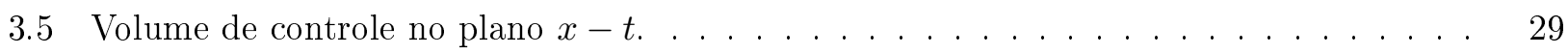

3.6 Evolução espacial das soluções numéricas da equação de Burgers 1D calculadas com TDPUS-C3. Euler explícito na parte temporal (não TVD). . . . . . . . . . . . . . . . 34

3.7 Soluções numéricas da equação de Burgers 1D calculadas com TDPUS-C3. SSP-RK3 na parte temporal (TVD). . . . . . . . . . . . . . . . . . . . . . . .

4.1 Célula computacional da malha deslocada. A variável $\phi$ representa $p_{e}, \kappa, \varepsilon, \nu_{t}, \tau_{p_{x x}}$,

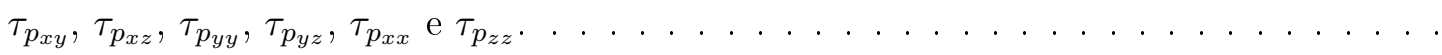




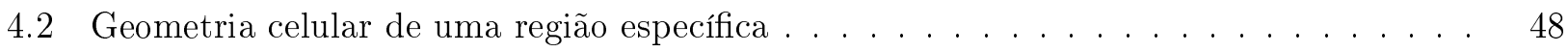

4.3 Ilustração de uma superfície planar inclinada formando angulo de $45^{0}$ com o vetor normal

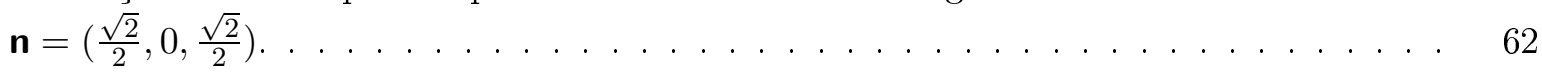

4.4 Perfil típico da camada limite turbulenta. . . . . . . . . . . . . . 63

4.5 Ilustração das células adjacentes ao contorno rígido. . . . . . . . . . . . . . . . 64

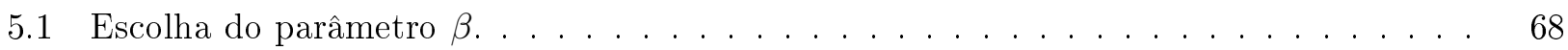

5.2 Teste 1: soluções numéricas do problema de advecção 1D com TDPUS-C3, ADBQUICKEST, ARORA-ROE, CUBISTA e TOPUS. $t=1, N=200$ e $C F L=0.5$. . .

5.3 Teste 1: soluções numéricas do problema de advecção 1D com TDPUS-C3, ADBQUICKEST, ARORA-ROE, CUBISTA e TOPUS. $t=20, N=200$ e $C F L=0.5$. . 70

5.4 Teste 3: análise TVD para o esquema TDPUS-C3. . . . . . . . . . . . . 73

5.5 Teste 4: soluções numéricas da equação invíscida de Burgers obtidas com o TDPUS-C3 antes e depois da formação do choque. . . . . . . . . . . . . . . . .

5.6 Teste 4: soluções numéricas da equação inviscida de Burgers depois do choque calculadas com TDPUS-C3, ADBQUICKEST, ARORA-ROE, CUBISTA e TOPUS. . . .

5.7 Teste 5: soluções numéricas ao longo do plano $x=y$ da equação de Burgers 2D com TDPUS-C3, ADBQUICKEST, ARORA-ROE, CUBISTA e TOPUS. $t=0.5 / \pi$, $160 \times 160$ células computacionais e $C F L=0.4 \ldots \ldots \ldots \ldots \ldots$

5.8 Teste 5: soluções numéricas ao longo do plano $x=y$ da equação de Burgers $2 \mathrm{D}$ com TDPUS-C3, EPUS, SDPUS-C1 e TOPUS. $t=0.5 / \pi, 160 \times 160$ células computacionais

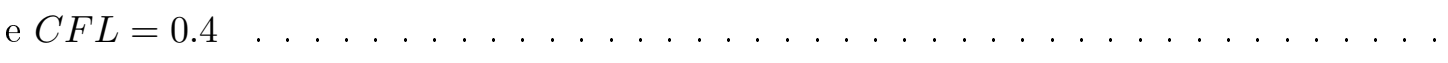

5.9 Teste 6: soluções numéricas para a equação não linear de Buckley-Leverett. $t=0.4$, $C F L=0.4$ e $N=200$.

5.10 Teste 6: soluções numéricas da equação de Buckley-Leverett. Região do choque $x \approx$ -0.077 e da descontinuidade de contato $x \approx 0.64$.

5.11 Ilustração esquemática de um escoamento com superfície livre sobre a influência da

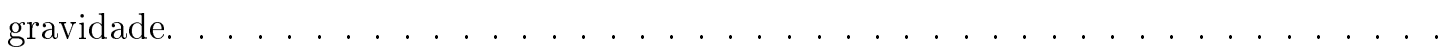

5.12 Teste 7: soluções numéricas com TDPUS-C3 e a solução exata para o nível da água $h$ no problema de ruptura da barragem. $N=400$ e $C F L=0.91$.

5.13 Teste 7: comparação entre as soluções numéricas para o nível da água $h$ com TDPUS-C3, ADBQUICKEST, ARORA-ROE, CUBISTA, TOPUS e a solução exata. $t=10, N=$

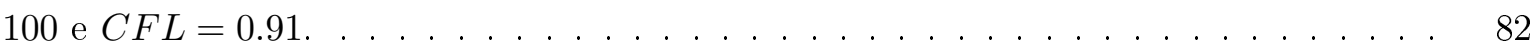

5.14 Teste 8: nível da água $h+B(x)$ e a vazão $h u_{1}$ (b). $t=200, N=200$ e $C F L=0.9 . \quad$. 82

5.15 Teste 9: ruptura de uma barragem circular. Soluções numéricas para o nível da água $h$ ao longo do eixo $x(y=0)$ obtidos com TDPUS-C3, FORCE, GODUNOV-HLL. . .

5.16 Teste 9: ruptura de uma barragem circular. Evolução espacial das soluções numéricas obtidas com TDPUS-C3 para o nível da água $h$ ao longo do eixo $x(y=0) \ldots \ldots$.

5.17 Teste 10: comparação entre as soluções numéricas da densidade $\rho$ para o problema de Shu-Osher com TDPUS-C3, ADBQUICKEST, ARORA-ROE, CUBISTA e TOPUS. 
5.18 Teste 10: evolução espacial das soluções numéricas da densidade $\rho$ para o problema de Shu-Osher com TDPUS-C3, ADBQUICKEST, ARORA-ROE, CUBISTA e TOPUS.

5.19 Teste 10: soluções numéricas da densidade $\rho$ problema de Shu-Osher nas regiões NF, FF, EW e TN.

5.20 Teste 11: comparação entre as soluções numéricas da densidade $\rho$ para o problema de Woodward-Colella obtidas com TDPUS-C3, ADBQUICKEST, ARORA-ROE, CUBISTA e TOPUS. . . . . . . . . . . . . . . . . . . .

5.21 Teste 11: comparação entre as soluções numéricas da densidade $\rho$ para o problema de Woodward-Colella com TDPUS-C3, EPUS, SDPUS-C1 e TOPUS.

5.22 Teste 11: problema de Woodward-Colella. Evolução temporal da densidade $\rho$ e velocidade $u_{1}$ das soluções numéricas obtidas com TDPUS-C3 e SUPERBEE.

5.23 Teste 11: problema de Woodward-Colella. Evolução temporal da densidade $\rho$ e velocidade $u_{1}$ obtidas com TDPUS-C3 e SUPERBEE. . . . . . . . . . . . . . . . .

5.24 Teste 13: soluções numéricas da densidade $\rho$ para o problema DMR com ADBQUICKEST na malha uniforme $\delta x=1 / 400$ e WENO5-LW4

5.25 Teste 13: ampliação da região principal das soluções numéricas para a densidade $\rho$ para o problema DMR com TDPUS-C3, ADBQUICKEST e ARORA-ROE. $t=0.2$.

5.26 Teste 13: ampliação da região principal das soluções numéricas para a densidade $\rho$ do problema DMR no tempo $t=0.2$ calculadas com CUBISTA e TOPUS. 30 linhas de contorno de $\rho=1.5$ até $\rho=22.97$, com malhas uniformes $\delta x=1 / 100,1 / 200,1 / 400$, $t=0.2$ e $C F L=0.6 \ldots \ldots \ldots \ldots \ldots \ldots \ldots \ldots$

5.27 Teste 13: ampliação da região principal das soluções numéricas para a densidade $\rho$ para o problema DMR calculada com SUPERBEE e $\delta x=1 / 200$ e WENO5 e $\delta x=1 / 960$. $t=0.2$

5.28 Teste 14: condições iniciais do problema das instabilidades de Rayleigh-Taylor. . . . .

5.29 Teste 14: soluções numéricas para a densidade $\rho$ para o problema das instabilidades de Rayleigh-Taylor com TDPUS-C3, ADBQUICKEST, ARORA-ROE, CUBISTA, TOPUS e SUPERBEE e WENO5 com $\delta x=1 / 960 \ldots \ldots \ldots \ldots \ldots \ldots \ldots$

5.30 Teste 14: Instabilidades de Rayleigh-Taylor. Perfil da energia cinética em $y=4 x$ no tempo $t=1.95$ com TDPUS-C3, ADBQUICKEST, ARORA-ROE, CUBISTA, TOPUS e SUPERBEE.

5.31 Teste 14: soluções numéricas para a densidade $\rho$ para o problema das instabilidades e Rayleigh-Taylor com TDPUS-C3, EPUS, SDPUS-C1 e TOPUS com $\delta x=1 / 960$.

5.32 Teste 15: soluções numéricas da densidade $\rho$ para o problema da MHD 1D com TDPUS-C3.

5.33 Teste 16: superfície da densidade $\rho$ para o problema de Orszag-Tang. . . . . . . . . 104

5.34 Teste 18: perfil da pressão $p$ ao longo da linha $y=0.625 \pi \ldots \ldots \ldots 4$

6.1 Jato de fluido incidente sobre uma superfície rígida . . . . . . . . . . . . . 106

6.2 Comparação das soluções numéricas em diferentes malhas para o problema do jato livre 2 D sobre uma superfície rígida no regime laminar. . . . . . . . . . . . . . . . 107 
6.3 Contorno da velocidade $u$ para o problema do jato livre 2D sobre uma superfície rígida. 108

6.4 Contorno da velocidade $v$ para o problema de jato livre 2D sobre uma superfície rígida no regime laminar. . . . . . . . . . . . . . . . . . . . . . . 109

6.5 Contorno da pressão $p$ para o problema do jato livre 2D sobre uma superfície rígida. . 109

6.6 Comparação entre as soluções numéricas da altura $h$ obtidas com TDPUS-C3 e analítica para o problema de simetria radial. . . . . . . . . . . . . . . . . . 110

6.7 Ressalto hidráulico circular em distintos ângulos simulado pelo esquema TDPUS-C3. 111

6.8 Ilustração esquemática do colapso de um bloco de fluido. . . . . . . . . . . . . . . . 111

6.9 Soluções numéricas para o espalhamento horizontal, $x_{\text {max }}$, obtidas com TDPUS-C3 para o problema do colapso de um bloco de fluido 3D no regime laminar. . . . . . . . . 112

6.10 Contorno da velocidade $u$ para o problema do colapso de um bloco de fluido 3D no regime laminar com TDPUS-C3. . . . . . . . . . . . . .

6.11 Contorno da velocidade $w$ para o problema do colapso de um bloco de fluido 3D no regime laminar com TDPUS-C3.

6.12 Contorno da pressão $p$ para o problema do colapso de um bloco de fluido 3D no regime laminar com TDPUS-C3. . . . . . . . . . . . . . . . . . . . . . 114

6.13 Estruturas dos jatos oscilantes viscosos segundo Cruickshank e Munson (1981). . . . . 115

6.14 Geometria para o problema do jato circular e planar. . . . . . . . . . . . . . . 115

6.15 Evolução temporal do jato circular oscilante. Teste 1 . . . . . . . . . . . . . 118

6.16 Evolução temporal do jato circular oscilante. Teste 2 . . . . . . . . . . . . . . . 119

6.17 Contorno da velocidade $u, v$ e da pressão $p$ do jato circular oscilante. Teste 2. $\quad$. . 120

6.18 Evolução temporal do jato planar oscilante com TDPUS-C3. $t=0.2 s, 0.4 s$ e $0.6 s$. . 121

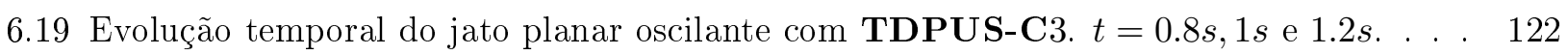

6.20 Contornos das velocidades $u, w$ e a pressão $p$ do jato planar oscilante. . . . . . . . . 123

6.21 Comparação entre as soluções numéricas e analítica para o problema do jato livre 2D sobre uma superfície rígida no modelo $\kappa-\varepsilon$ padrão associado ao TDPUS-C3. . . . .

6.22 Comparação entre as soluções numéricas e analítica para o problema do jato livre 2D sobre uma superfície rígida impermeável no modelo $R N G \kappa-\varepsilon$ associado ao TDPUS-C3.126

6.23 Comparação entre as soluções numéricas obtidas com TDPUS-C3 nos modelos $\kappa-\varepsilon$ padrão e $R N G \kappa-\varepsilon$ associados ao TDPUS-C3, e analítica do problema do jato livre 2D sobre uma superfície rígida impermeável. Malha I. . . . . . . . . . . . . .

6.24 Contorno da velocidade $u$ para o problema do jato livre 2D sobre uma superfície rígida impermeável no modelo $\kappa-\varepsilon$ padrão associado ao TDPUS-C3. Malha III. . . . . . . .

6.25 Contorno da velocidade $v$ para o problema do jato livre 2D sobre uma superfície rígida impermeável no modelo $\kappa-\varepsilon$ padrão associado ao TDPUS-C3. Malha III. . . . . . . .

6.26 Contorno da pressão $p$ para o problema do jato livre 2D sobre uma superfície rígida impermeável no modelo $\kappa-\varepsilon$ padrão associado ao TDPUS-C3. Malha III. . . . . . .

6.27 Contorno da velocidade $u$ para o problema do jato livre 2D sobre uma superfície rígida impermeável no modelo $R N G \kappa-\varepsilon$ padrão associado ao TDPUS-C3. Malha III. . .

6.28 Contorno da velocidade $v$ para o problema do jato livre 2D sobre uma superfície rígida impermeável no modelo $R N G \kappa-\varepsilon$ padrão. Malha III. 
6.29 Contorno da pressão $p$ para o problema do jato livre 2D sobre uma superfície rígida impermeável no modelo $R N G \kappa-\varepsilon$ padrão associado ao TDPUS-C3. Malha III. . .

6.30 Soluções numéricas obtidas com TDPUS-C3 (extraídas no plano $y z$ na posição $x=$ 0.056) para o problema do jato livre $3 \mathrm{D}$ com os modelos de turbulência $\kappa-\varepsilon$ padrão e $R N G \kappa-\varepsilon$ associados com TDPUS-C3, comparadas com as soluções viscosa e não viscosa de Watson (1964).

6.31 Comparação entre as soluções numéricas (extraídas no plano yz na posição $x=0.056$ ) obtidas com TDPUS-C3 e as analíticas, não viscosa e viscosa de Watson, para o problema do jato livre 3D com as modelagens de turbulência $\kappa-\varepsilon$ padrão e $R N G \kappa-\varepsilon$ associados com TDPUS-C3. . . . . . . . . . . . . . .

6.32 Evolução temporal do problema do jato livre 3D sobre uma superfície rígida impermeável usando a associação $R N G \kappa-\varepsilon$-TDPUS-C3. Malha II. . . . . . . . . . . . . . .

6.33 Contorno da velocidade $u$ para o problema do jato livre 3D sobre uma superfície rígida impermeável usando as associações $\kappa-\varepsilon$ padrão-TDPUS-C3 e $R N G \kappa-\varepsilon$-TDPUS-C3. Malha II.

6.34 Contorno da velocidade $w$ para o problema do jato livre 3D sobre uma superfície rígida impermeável usando as associações $\kappa-\varepsilon$ padrão-TDPUS-C3 e $R N G \kappa-\varepsilon$-TDPUS-C3. Malha II.

6.35 Contorno da pressão $p$ para o problema do jato livre 3D sobre uma superfície rígida impermeável usando as associações $\kappa-\varepsilon$ padrão -TDPUS-C3 e $R N G \kappa-\varepsilon$-TDPUS-C3. Malha II.

6.36 Ilustração do problema de colapso de fluido e interação da superfície livre com um obstáculo.

6.37 Evolução temporal das superfícies livres usando a combinação $R N G \kappa-\varepsilon$-TDPUS-C3 do problema de colapso de fluido e interação da superfície livre com um obstáculo. Vista lateral.

6.38 Evolução temporal das superfícies livres usando a combinação $R N G \kappa-\varepsilon$-TDPUS-C3 do problema de colapso de fluido e interação da superfície livre com um obstáculo. Vista de cima . . . . . . . . . . . . . . . . . . . . . . .

6.39 Geometria para o modelo SXPP. 136

6.40 Modelo SXPP. Resultados obtidos com TDPUS-C3. $R e=0.96, W e=0.1, \varrho=0.5$, $\vartheta=0.9, \gamma=0.1, Q=1$.

6.41 Modelo SXPP. Resultados obtidos com TDPUS-C3. $R e=0.96, W e=0.1, \varrho=0.01$, $\vartheta=0.3, \gamma=0.8$.

6.42 Modelo SXPP. Resultados obtidos com TDPUS-C3. $R e=0.64, W e=0.1, \varrho=0.5$, $\vartheta=0.9, \gamma=0.1, Q=1$.

6.43 Modelo SXPP. Resultados obtidos com TDPUS-C3. $R e=0.32, W e=0.1, \varrho=0.5$, $\vartheta=0.9, \gamma=0.1$.

6.44 Modelo SXPP (Extraído de Figueiredo (2011)). Resultados obtidos com CUBISTA. $R e=0.32, W e=0.1, \varrho=0.5, \vartheta=0.9, \gamma=0.1$ e $Q=1$. 


\section{Lista de Tabelas}

2.1 Alguns esquemas em variáveis não normalizadas e suas correspondentes expressões em variáveis normalizadas. . . . . . . . . . . . . . . . . . . . . 10

2.2 Diferentes valores do parâmetro $\beta$ para o esquema TDPUS-C3 . . . . . . . . . . . 22

4.1 Parâmetros do modelo $\kappa-\varepsilon$ padrão de turbulência. . . . . . . . . . . . . . . . . . 41

4.2 Parâmetros do modelo $R N G \kappa-\varepsilon$ de turbulência. . . . . . . . . . . . . . . . . 41

5.1 Escolha do parâmetro $\beta$. Teste de convergência para TDPUS-C3 $\operatorname{com} \beta=64,266$, 526 e 567.25 da equação de Burgers $1 \mathrm{D}$ no tempo $t=0.33$ e $C F L=0.5$. . . . . . . .

5.2 Teste 1: teste de convergência do experimento de Zalesak com TDPUS-C3, ADBQUICKEST, ARORA-ROE, CUBISTA e TOPUS no tempo $t=1$. SSP-RK3 na parte

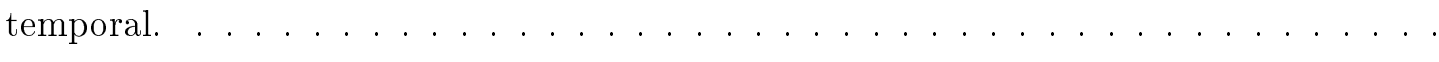

5.3 Teste 1: teste de convergência do experimento de Zalesak com TDPUS-C3, ADBQUICKEST, ARORA-ROE, CUBISTA e TOPUS no tempo $t=20$. SSP-RK3 na

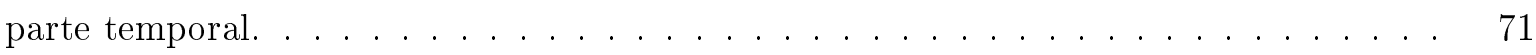

5.4 Tempo de computação por iteração para o Teste $1 . \ldots \ldots$. . . . . . . . . . 71

5.5 Teste 2: teste de convergência obtido com TDPUS-C3. $t=1$ e $C F L=0.5 \ldots$. . . 72

5.6 Teste 5: teste de convergência para a equação de Burgers 2D com TDPUS-C3, ADBQUICKEST, ARORA-ROE, CUBISTA e TOPUS na norma $L_{1} \ldots \ldots \ldots$. . . . . . 74

5.7 Teste 5: teste de convergência para a equação de Burgers 2D com TDPUS-C3, ADBQUICKEST, ARORA-ROE, CUBISTA e TOPUS na norma $L_{\infty} . \ldots \ldots$. . . . . . 75

5.8 Teste 5: teste de convergência para a equação de Burgers 2D com TDPUS-C3, EPUS, SDPUS-C1 e TOPUS na norma $L_{1} . t \approx 0.5 / \pi$ e $C F L=0.4 \ldots \ldots \ldots \ldots \ldots$

5.9 Teste 5: teste de convergência para a equação de Burgers 2D com TDPUS-C3, EPUS, SDPUS-C1 e TOPUS na norma $L_{\infty} . t \approx 0.5 / \pi$ e $C F L=0.4 \ldots \ldots \ldots \ldots$

5.10 Teste 12: teste de convergência da densidade $\rho$ para as equações não lineares de Euler 2D com TDPUS-C3, ADBQUICKEST, ARORA-ROE, CUBISTA e TOPUS na norma $L_{1} . t=2$ e $C F L=0.475$. 
5.11 Teste 12: teste de convergência da densidade $\rho$ para as equações não lineares de Euler 2D com TDPUS-C3, ADBQUICKEST, ARORA-ROE, CUBISTA e TOPUS na norma $L_{\infty} . t=2$ e $C F L=0.475 \ldots \ldots \ldots \ldots \ldots \ldots$

5.12 Teste 18: teste de convergência da densidade $\rho$ para o problema de Orszag-Tang com TDPUS-C3, ADBQUICKEST, ARORA-ROE, CUBISTA e TOPUS na norma $L_{1} \ldots 104$

6.1 Dados da simulação do jato livre 2D no regime laminar. . . . . . . . . . . . . . 107

6.2 Dados da simulação do jato livre 2D no regime laminar em simetria radial. . . . . . . . 108

6.3 Estimativas teóricas e numéricas nas malhas I, II e III para o raio do salto hidráulico. 110

6.4 Dados da simulação do colapso de um bloco de fluido. . . . . . . . . . . . . . . . 112

6.5 Dados da simulação do jato circular oscilante. Teste 1. . . . . . . . . . . . . . 116

6.6 Dados da simulação do jato circular oscilante. Teste $2 \ldots \ldots$. . . . . . . . . . . . 116

6.7 Dados da simulação do jato circular oscilante. Teste $3 . \quad \ldots \ldots \ldots$. . . . . . . . . 117

6.8 Dados da simulação do jato livre 2D no regime turbulento. . . . . . . . . . . . . . . . 124

6.9 Dados da simulação do jato livre 3D no regime turbulento. . . . . . . . . . . . . . 130

6.10 Dados da simulação do colapso de fluido e interação da superfície livre com um obstáculo.134

6.11 Parâmetros dos experimentos para o modelo SXPP . . . . . . . . . . . . . 138 


\section{Lista de Abreviaturas}

ADBQUICKEST - Adaptive Bounded QUICKEST

BEM - Boundary Element Method

CBC - Convective Boundedness Criterion

CDS - Central Difference Scheme

CFL - Courant-Friedrich-Levy

CFD - Computational Fluid Dynamics

CHJ - Circular Hydraulic Jump

CLAWPACK - Conservation Laws Package

CUBISTA - Convergent and Universally Bounded Interpolation Scheme for Treatment Interpolation

DC - Diferencias Centrais

DF - Dinâmica de Fluidos

DFC - Dinâmica de Fluidos Computacional

DNS - Direct Numerical Simulation

DVN - Diagrama de Variáveis Normalizadas

EDO - Equação Diferencial Ordinária

EDPs - Equações com Derivadas Parciais

ENO - Essentially Non-Oscillatory

EPUS - Eight Degree Upwind Polynomial Scheme

ECBC - Extended Convective Boundedness Criterion

FORCE - First-Order Centred

FOU - First Order Upwind

Fr - Número de Froude

GENSMAC - Generalized-Simplified-Marker-and-Cell

HLL - Harten-Lax-van Leer

HLLE - Harten-Lax-van Leer-Einfeldt

ICMC - Instituto de Ciências Matemáticas e de Computação

LMACC - Laboratório de Matemática Aplicada e Computação Científica

LW - Lax-Wendroff

LES - Large-Eddy Simulation

LF - Limitadores de Fluxo

MAC - Marker and Cell

MC - Monotonized Central

MFIX - Multiphase Flow with Interphase eXchanges

MHD - Magnetohidrodinâmica 
MUSCL - Monotone Upstream-Centered Schemes for Conservation Laws

PPM - Piecewise Parabolic Method

QUICK - Quadratic Upstream Interpolation for Convective Kinematics

QUICKEST - Quadratic Upstream Interpolation for Convective Kinematics with Estimated Streaming Terms

Re - Número de Reynolds

RNG - Renormalization Group

SHARP - A Simple High-Accuracy Resolution Program

SMAC - Simplified Marker-And-Cell

SDPUS-C1 - Six-Degree Polynomial Upwind Schemes C1

SMART - Sharp and Monotonic Algorithm for Realistic Transport

SOU - Second Order Upwind

SPH - Smoothed Particle Hydrodynamics

SSP-RK3 - Strong Stability Preserving Runge-Kutta Third Order

SXPP - Single eXtended Pom Pom

TDPUS-C3 - Tenth Degree Polynomial Upwind Scheme C3

TOPUS - Third-Order Polynomial Upwind Scheme

TV - Total Variation

TVD - Total Variation Diminishing

URANS - Unsteady Reynolds-Averaged Navier-Stokes

USP - Universidade de São Paulo

VN - Variaveis Normalizadas

VONOS - Variable-Order Non-Oscilatory Scheme

WACEB - Weighted-Average Coefficient Ensuring Boundedness

WB - Warming-Beam

We - Número de Weisemberg

WENO - Weighted Essentially Non-Oscillatory 


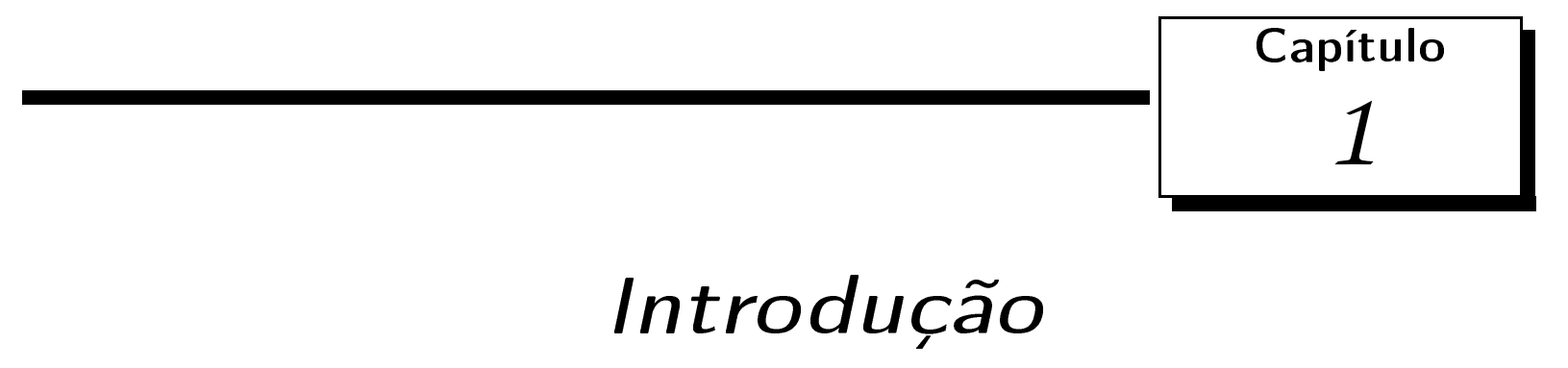

\subsection{Tema da pesquisa}

A maioria dos problemas físicos modelados por EDPs em DFC, que vão desde leis de conservação hiperbólicas com termos-fonte até equações de Navier-Stokes a altos valores do número de Reynolds, é dominada por convecção; e, portanto, propensa a formação de soluções descontínuas e estruturas vorticais. A dominância dos termos convectivos (em geral não lineares) dificultam sobremaneira a derivação de soluções numéricas para tais sistemas. Em função disso, considera-se crucial propor técnicas computacionais especializadas para resolver esses problemas complexos. De interesse particular no presente trabalho estão as aproximações dos termos convectivos por esquemas upwind de alta resolução e a implementação de modelagens $\kappa-\varepsilon$ de turbulência. As propriedades desejadas dos métodos de aproximação aqui investigados são: (i) boa precisão e robustez; (ii) limitação da solução numérica sem oscilações não físicas e com pouca introdução de viscosidade numérica; e (iii) simplicidade na implementação e baixo custo computacional.

\subsection{Desenvolvimento de modelagens numéricas}

Desde os anos 50, uma grande quantidade de modelagens numéricas para os termos convectivos tem sido concebida com o propósito de simular computacionalmente problemas complexos em DFC. A maior dificuldade que tem motivado a pesquisa na área é o problema de representar bem fortes choques, descontinuidades de contato e estruturas vorticais que aparecem nas soluções numéricas. As primeiras tentativas bem sucedidas para estes problemas foram propostas por VonNeumann e Richtmyer (1950), Courant et al. (1952) e Spalding (1972), utilizando métodos de primeira ordem de precisão local. Entretanto, certas desvantagens intrínsecas a esses métodos clássicos, tais como suavização da solução em regiões de altos gradientes, têm direcionado a pesquisa na área para a derivação de soluções simples, mais precisas e/ou incondicionalmente estáveis; apareceu até um teorema devido a Godunov (1959) afirmando que esquemas lineares que não geram oscilações numéricas só podem ser de primeira ordem. A cura para isto tem sido o uso de esquemas convencionais, tais como 
diferenças centrais (DC), upwind de segunda ordem (SOU) (Warming e Beam, 1976), QUICK (Leonard, 1979) e QUICKEST (Leonard, 1988a), entre outros. No entanto, sob condições severas de convecção, tais como o movimento turbulento, esses esquemas inevitavelmente produzem soluções oscilatórias (não monotônicas) em regiões onde as variáveis convectadas experimentam gradientes elevados.

Com o objetivo de superar os defeitos associados aos esquemas convencionais citados anteriormente, um número substancial de esquemas monotônicos tem surgido na literatura especializada. Como exemplo pode-se citar a classe MUSCL de van Leer (1979) e os esquemas MINMOD de Roe (1986), SMART de Gaskell e Lau (1988), SHARP de Leonard (1988b), WACEB de Song et al. (2000), CUBISTA de Alves et al. (2003), ARORA-ROE de Arora e Roe (1997) e ADBQUICKEST de Ferreira et al. (2009). A principal vantagem desses esquemas é que eles capturaram bem soluções suaves daquelas contaminadas com oscilações não físicas e, ao mesmo tempo, melhoraram a convergência. Deve-se observar também que esses esquemas (alguns deles ao menos), embora funcionando bem em alguns problemas, não produzem soluções limitadas nas situações com choques, descontinuidades de contato (ver, por exemplo, Kuan e Lin (2000) e Lin e Lin (1997)) e/ou em simulações de escoamentos viscoelásticos com modelos constitutivos hiperbólicos (Xue et al., 2002). Em Lin e Chieng (1991), os autores observaram que os esquemas SMART e SHARP, embora preservem alta precisão, produzem altos níveis de oscilações no caso do problema do tubo de choque de Sod (1978). Em Alves et al. (2000), com o uso de esquemas upwind, os autores simularam uma série de escoamentos viscoelásticos e observaram que os cálculos não convergiram com o refinamento de malha. Em Baxevanou e Fidaros (2008) há uma associação de esquemas upwind TVD com a modelagem $\kappa-\varepsilon$ padrão cujos resultados apontaram deficiências do esquema upwind devido a Roe-Sweby, mas com o emprego do limitador de fluxo MINMOD, essa associação apresentou melhorias quando comparado aos esquemas de primeira ordem, DC e QUICK; e estes últimos levando à divergência dos cálculos em problemas onde a convecção é dominante.

Recentemente tem aparecido na literatura alguns trabalhos associando esquemas upwind (usualmente os esquemas DC, FOU, SOU e QUICK) com a modelagem $R N G \kappa-\varepsilon$ de (Yakhot e Orszag, 1986). Em Fudihara et al. (2007), usando os esquemas FOU e SOU, foi feito um estudo numérico da aerodinâmica de um forno com um queimador de bloco; em Wang e Wang (2011) é apresentada uma comparação entre soluções numéricas, com o uso do esquema DC e as modelagens $\kappa-\varepsilon$ padrão, $R N G \kappa-\varepsilon$ e Realizable $\kappa-\varepsilon$ (Shih et al., 1995), para o problema do jato livre 2D sobre uma superfície rigida; e em Ghadimi et al. (2012), os autores utilizaram o código comercial Fluent@ incrementado com o esquema DC para simular a transferência de calor no fluxo de ar em janelas. Observa-se, portanto, uma tendência atual na literatura em se usar os esquemas convectivos FOU, DC e QUICK em problemas envolvendo turbulência. Assim, a necessidade de um esquema de convecção tipo upwind que proporciona boa precisão, limitação da solução numérica (sem oscilações não físicas e com pouca introdução de viscosidade numérica) e simplicidade na implementação para aproximar termos convectivos de leis de conservação e problemas relacionados em DFC continua a estimular a pesquisa científica em várias áreas da engenharia. Dentro deste cenário, e no contexto dos critérios de limitação TVD e $\mathrm{CBC}$, três novos esquemas upwind (e correspondentes limitadores de fluxo) apareceram na literatura: o TOPUS de Ferreira et al. (2012), o SDPUS-C1 de Lima et al. (2012) e, mais recentemente, o esquema EPUS de Corrêa et al. (2012). O esquema TOPUS foi proposto utilizando parte de um polinômio de grau quatro e mostrou ser uma boa ferramenta para uma variedade de problemas da dinâmica dos 
fluidos; no entanto o TOPUS não se reduz a uma função suave nos pontos 0 e 1 dentro do diagrama de variáveis normalizadas de Leonard (1988a); isso, segundo Lin e Chieng (1991), pode gerar problemas de convergência em problemas complexos, como é o caso das instabilidades de Rayleigh-Taylor e escoamentos incompressíveis envolvendo superfícies livres móveis, e muitas outras aplicações. Os esquemas SDPUS- $C 1$ e EPUS, por outro lado, são de classe $C^{1}$ e $C^{2}$ nesses pontos, respectivamente.

A derivação de um novo esquema convectivo TVD/CBC universal, para reconstruções (upwind) de fluxos numéricos, que possua uma molécula computacional simples (envolvendo no máximo três pontos de malha por fluxo numérico), que satisfaz propriedades de diferenciabilidade superior a dois e que seja uma alternativa à classe de esquemas upwind de captura de choques é uma tema de relevância em DFC e contemplado nesta tese. Vale reconhecer que há também uma classe importante de esquemas sofisticados, os bem conhecidos ENO/WENO e suas variações (ver, por exemplo, Qiu e Shu (2011) e Wan et al. (2012)), para simulações de escoamentos compressíveis; essa classe tem proporcionado boa precisão local com pouca viscosidade numérica, mas é difícil de implementar e relativamente pobre em malhas grosseiras.

\subsection{Motivação, objetivo e contribuição do presente estudo}

O interesse crescente de soluções aproximadas para equações de conservação da DFC deriva da demanda por modelagens simples, robustas, baratas e capazes de prever campos de escoamentos de fluidos contendo fortes choques, descontinuidades de contato e/ou estruturas vorticais, como acontece nas equações de Euler da dinâmica dos gases e nas equações de Navier-Stokes sob forte influência dos termos não lineares. Nos últimos anos, os pesquisadores do LMACC do ICMC-USP têm-se dedicado ao desenvolvimento de métodos numéricos para simular escoamentos incompressíveis newtonianos e não newtonianos com superfícies livres móveis. Em particular, várias técnicas inovadoras têm sido desenvolvidas por esses pesquisadores, dentre elas destacam-se:

- desenvolvimento e implementação de métodos numéricos para escoamentos de fluidos newtonianos em uma ampla faixa do número de Reynolds (Ferreira et al., 2012);

- implementação de modelagens de turbulência $\kappa-\varepsilon$ padrão (Ferreira et al., 2013);

- análise, implementação e validação de modelos reológicos a uma variada gama de números de Weisemberg (Oishi et al., 2012, Tomé et al., 2012); e

- simulação computacional de escoamentos complexos, tais como bifásicos fluido-fluido com superfícies livres móveis (Santos et al., 2012, Lima et al., 2012) e gás-sólido num leito fluidizado (Corrêa et al., 2012).

Com o auxílio de esquemas upwind de alta resolução de classe de diferenciabilidade 1 e 2, esses avanços têm sido parcialmente incorporados aos códigos CLAWPACK de LeVeque (2012), Freeflow de Castelo et al. (2000) e MFiX (2011), permitindo a simulação de sistemas hiperbólicos de leis de conservação, de escoamentos incompressíveis de fluidos newtonianos em uma ampla faixa do número de Reynolds e de escoamentos reológicos 3D de interesse tecnológico na indústria de polímeros (ver McKee et al. (2008), Tomé et al. (2008), Ferreira et al. (2009), Carvalho et al. (2010), Queiroz e Ferreira (2010), Oishi et al. (2011), Lima et al. (2012) e Ferreira et al. (2012)). Entretanto, esses códigos são 
carentes de um esquema upwind de classe $C^{3}$ universal e uma modelagem $R N G \kappa-\varepsilon$ da turbulência. A associação desses duas modelagens (upwinding e $R N G \kappa-\varepsilon$ ) constitui, portanto, a motivação principal para o presente trabalho de tese.

O objetivo deste trabalho é o desenvolvimento e teste de um novo esquema convectivo upwind polinomial de grau dez de classe $C^{3}$, denominado TDPUS-C3. Outro objetivo é a associação do esquema TDPUS-C3 com modelagens $\kappa-\varepsilon$ (padrão e $R N G \kappa-\varepsilon$ ) para a simulação computacional de uma variedade de problemas complexos em DFC, usando a metodologia URANS. E a ênfase da tese é fornecer à literatura técnicas numéricas alternativas as quais podem ser utilizadas tanto em escoamentos compressíveis quanto em incompressíveis.

A contribuição do presente trabalho é como segue:

(i) introdução de um novo esquema convectivo upwind universal de classe $C^{3}$ (o TDPUS-C3) para a reconstrução de fluxos numéricos; com a expectativa de resolver problemas complexos em regiões suaves e altos gradientes com pontos extremos e descontinuidades;

(ii) adaptação do código CLAWPACK com o esquema TDPUS-C3 para resolver sistemas hiperbólicos de leis de conservação;

(iii) inclusão no ambiente de simulação Freeflow do novo esquema upwind para resolver escoamentos newtonianos e não newtonianos;

(iv) incorporação (análise e implementação) no sistema Freeflow da modelagem $R N G \kappa-\varepsilon$ e sua associação com o esquema TDPUS-C3 para resolver escoamentos incompressíveis turbulentos;

(v) disponibilização de uma variedade de dados de simulações numéricas $(\approx 24)$ para equações de conservação da DFC;

(vi) apresentação à literatura dos principais resultados numéricos oriundos deste trabalho de tese, cujos detalhes estão descritos no Apêndice A.

\subsection{Estrutura da tese e equipamentos utilizados}

O restante da tese está estruturada em sete capítulos descritos a seguir. No capítulo 2 apresenta-se uma breve descrição da teoria dos esquemas convectivos em variáveis normalizadas e correspondentes limitadores de fluxo. Nesse capítulo inclui-se ainda o desenvolvimento do novo esquema convectivo upwind TDPUS-C3. O capítulo 3 é reservado para a formulação de sistemas hiperbólicos e a metodologia de solução, incluindo o método de Godunov, o algoritmo de propagação das ondas e resolvedores de Riemann. No capítulo 4 estão descritos os conceitos básicos da formulação matemática de escoamentos incompressíveis newtonianos nos regimes laminar e turbulento e fluidos não newtonianos. Neste capítulo contempla-se também a discretização dos termos convectivos e das condições de contorno adotadas. O capítulo 5 contém resultados numéricos para sistemas hiperbólicos de leis de conservação 1D e 2D. Comparações com esquemas bem estabelecidos na literatura e com soluções analíticas são também realizadas. O capítulo 6 é destinado aos resultados de simulações numéricas de problemas complexos, tais como escoamentos incompressíveis newtonianos (nos regimes laminar e turbulento) e viscoelásticos. As considerações finais e trabalhos futuros são discutidos no capítulo 7. 
As simulações numéricas apresentadas ao longo desta tese foram rodadas nos seguintes computadores do LMACC do ICMC-USP:

- um computador Core Quad Q9650 3Ghz, 4Gb de memoria RAM, 500 Gb de disco rígido e sistema operacional Ubuntu 10.04, linux 2.6.32;

- uma estação de trabalho 8 x i7 Core i7 CPU-950 3.07Ghz, 16 Gb de memoria RAM, 1.6 Tb de disco rígido e sistema operacional Ubuntu 10.04, linux 2.6.35; e

- um cluster constituído de: 16 nós octos Xeon, cada máquina com dois processadores Quad Core Intel(R) Xeon(R) E5345 de 2.33GHz, 16 Gb de memoria RAM, 500 Gb de disco rígido. 


\section{Capítulo}

\section{Esquemas convectivos upwind}

Neste capítulo apresenta-se a base teórica para o desenvolvimento de esquemas convectivos tipo upwind que caracterizam-se por respeitar o sentido de propagação das informações do escoamento e o desenvolvimento do novo esquema convectivo TDPUS-C3.

\subsection{Motivação e aproximação upwind}

Em muitas aplicações, tais como poluição do ar, oceanografia e outras ciências físicas, é necessário calcular o transporte (ou conveç̧ão) de propriedades físicas (ou concentrações de constituintes) no escoamento de um fluido. Por exemplo, considere um fluido em movimento cuja velocidade do campo, em cada ponto do domínio, é conhecida, como ilustrado na Fig. 2.1.

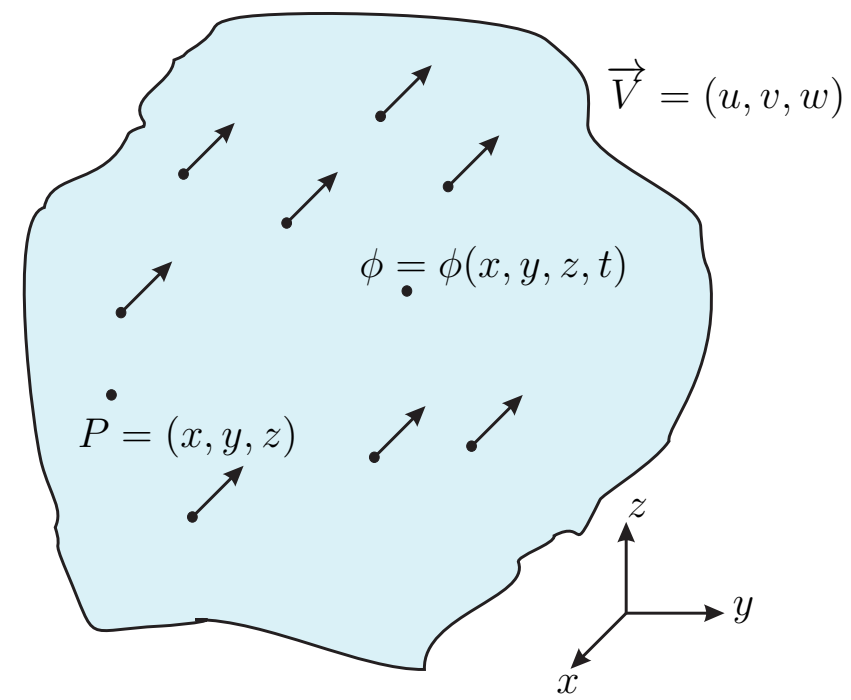

Figura 2.1: Representação esquemática do campo de velocidades de um escoamento de fluido.

Dado $P=(x, y, z)$, denota-se este campo de vetores por $\vec{V}=(u, v, w)^{T}$, em que $u=u(x, y, z, t)$, $v=v(x, y, z, t), w=w(x, y, z, t)$, sendo $u, v, w: \mathbb{R}^{4} \rightarrow \mathbb{R}$. Seja $\phi=\phi(x, y, z, t)$ uma variável 
convectada no escoamento; pode-se pensar, por exemplo, em uma gota de tinta espalhando-se no fluido ou no transporte de um poluente no escoamento. A equação que modela o transporte da variável $\phi$ em um campo de velocidade é, no caso 3D, dada por

$$
\frac{\partial \phi}{\partial t}+\frac{\partial(u \phi)}{\partial x}+\frac{\partial(v \phi)}{\partial y}+\frac{\partial(w \phi)}{\partial z}=0
$$

onde as componentes $u, v, w$, definidas previamente, são as velocidades de convecção da variável $\phi$ nas direções $x, y, z$, respectivamente.

Sem perda de generalidade, considera-se nesse estudo o caso 1D da equação (2.1) dada por

$$
\frac{\partial \phi}{\partial t}+\frac{\partial(u \phi)}{\partial x}=0, \quad 0 \leq t \leq T, \quad a \leq x \leq b, \quad T>0
$$

O problema definido por (2.2) com condição inicial $\phi(x, 0)=\phi_{0}(x)$ e condições de contorno $\phi(a, t)=\phi_{a}$ e $\phi(b, t)=\phi_{b}$, sendo $u$ a velocidade de convecção- campo este que geralmente não pode ser calculado analiticamente, requer aproximações numéricas para advecção de $\phi$. No caso em que $u=1$, a seguinte expressão é a solução analítica (ver Fig. 2.2)

$$
\phi(x, t)=\phi_{0}(x-t)
$$

Desta forma, uma informação transportada via advecção é transladada para direita quando a velocidade de convecção $u$ é positiva, e para esquerda quando negativa. A Fig. 2.2 ilustra o comportamento da solução analítica para $u>0$.

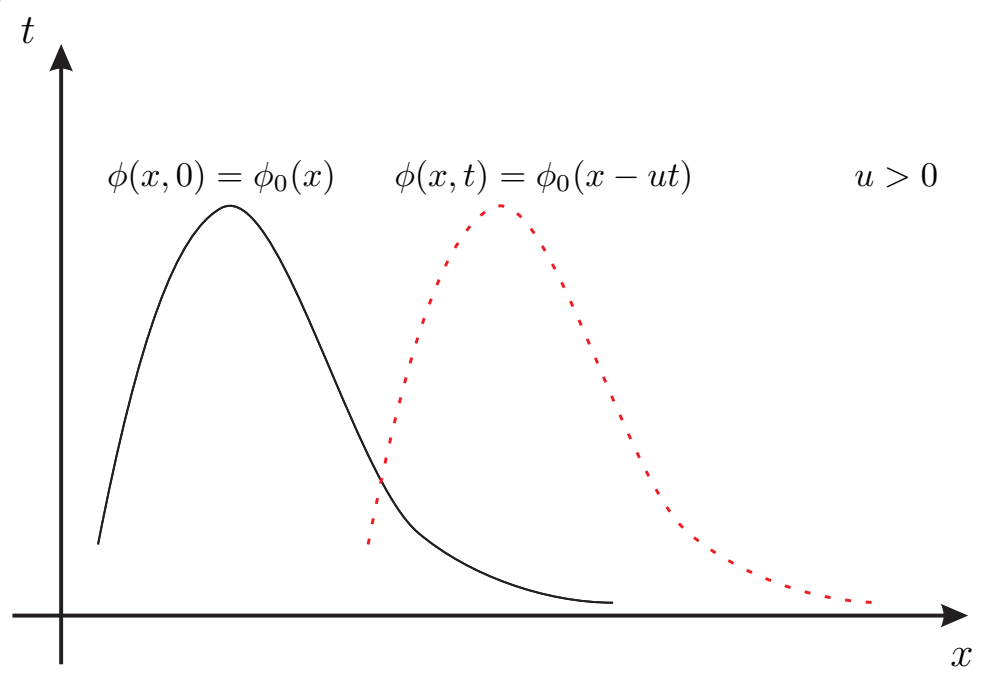

Figura 2.2: Solução analítica do problema de advecção com velocidade de convecção $u>0$.

O termo convectivo (2.2) avaliado num ponto $\mathcal{P}$ de uma malha estruturada, como indicado na Fig. 2.3 , é aproximado por

$$
\left.\frac{\partial(u \phi)}{\partial x}\right|_{\mathcal{P}} \approx \frac{\left.(u \phi)\right|_{f}-\left.(u \phi)\right|_{g}}{\delta x}=\frac{u_{f} \phi_{f}-u_{g} \phi_{g}}{\delta x}
$$

onde $\delta x$ é o espaçamento na direção $x . u_{f}$ e $u_{g}$ são as velocidades de convecção nas faces $f=i+1 / 2$ e $g=i-1 / 2$, respectivamente, as quais são definidas como as médias simples dos valores vizinhos, isto 
é $u_{f}=\left(u_{D}+u_{U}\right) / 2$ e $u_{g}=\left(u_{U}+u_{R}\right) / 2$.

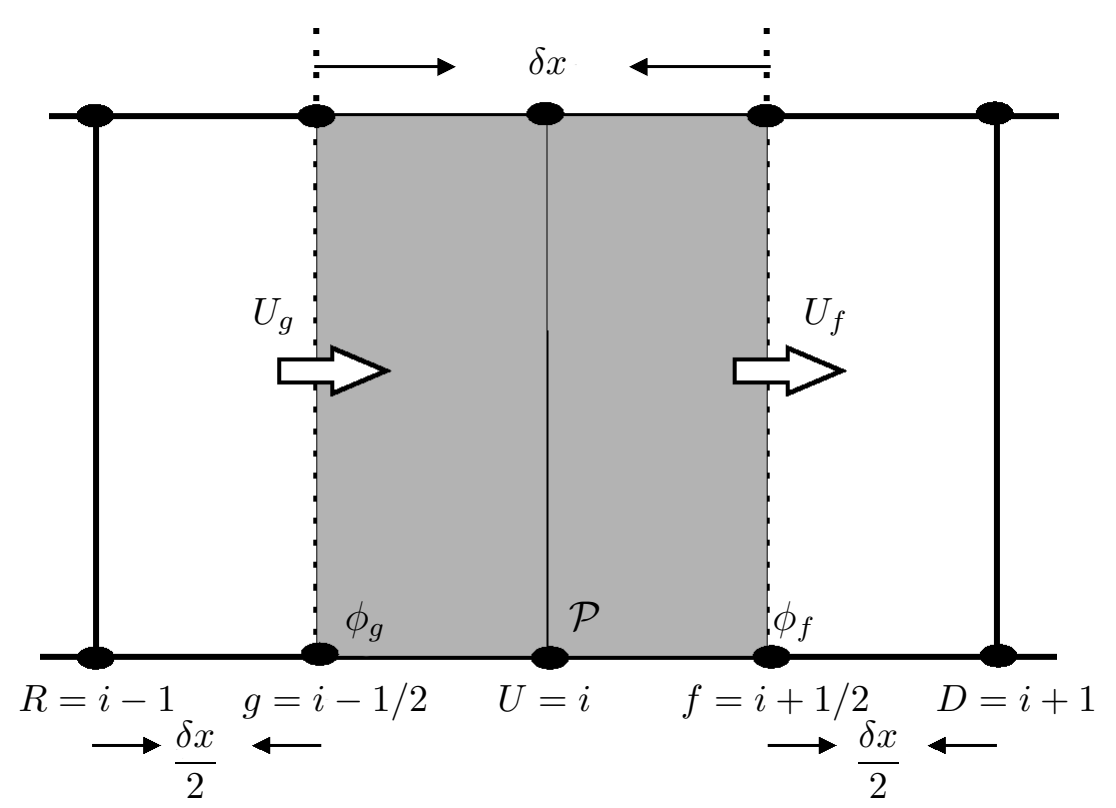

Figura 2.3: Molécula computacional da discretização dos termos convectivos no ponto $\mathcal{P}$.

Aproximações para a variável convectada $\phi$, em (2.4), nas faces $f$ e $g, \phi_{f}$ e $\phi_{g}$ (ou fluxos numéricos nestas faces), podem ser obtidas por interpolação upwind em função dos valores dessa variável nos seguintes pontos: o à jusante $D$, o à montante $U$ e o mais à montante $R$, como ilustrado na Fig. 2.3; e essas posições para interpolação são automaticamente definidas de acordo com os sinais das velocidades de convecção $U_{f}$ e $U_{g}$. Em síntese, uma aproximação (ou esquema) upwind para $\phi_{f}$ (um esquema para $\phi_{g}$ segue procedimentos similares) é da forma

$$
\phi_{f}=\phi_{f}\left(\phi_{D}, \phi_{U}, \phi_{R}\right)
$$

em que $\phi_{D}, \phi_{U}$ e $\phi_{R}$ são os valores de $\phi$ nos pontos $D, U$ e $R$, respectivamente. Para simplificar a relação funcional (2.5), Leonard (1988a) introduziu o conceito de variáveis normalizadas (VN) definido por

$$
\hat{\phi}_{(\cdot)}=\frac{\phi_{(\cdot)}-\phi_{R}}{\phi_{D}-\phi_{R}}
$$

com $\hat{\phi}_{D}=1$ e $\hat{\phi}_{R}=0$. A relação funcional (2.5) comumente denominada característica em variáveis normalizadas, passa então a ser representada como

$$
\hat{\phi}_{f}=\hat{\phi}_{f}\left(\hat{\phi}_{U}\right)
$$

Na Fig. 2.4 estão ilustrados, no diagrama de variáveis normalizadas (DVN), os esquemas clássicos FOU, SOU, CDS e QUICK. Nesta figura, a região hachurada correspondente à região de monotonici- 
dade para esquemas de segunda ordem de precisão local.

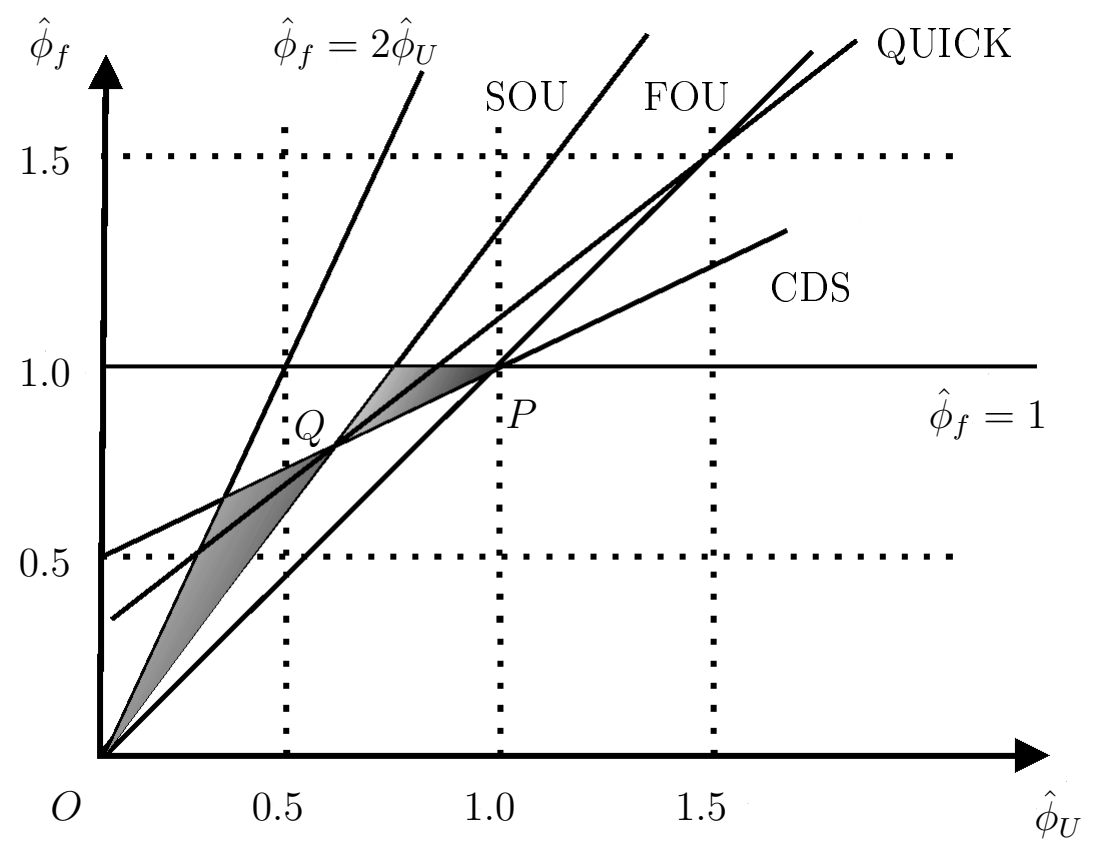

Figura 2.4: Região de monotonicidade para esquemas de segunda ordem no diagrama de variáveis normalizadas.

Tabela 2.1: Alguns esquemas em variáveis não normalizadas e suas correspondentes expressões em variáveis normalizadas.

\begin{tabular}{ccc}
\hline \hline Esquema & Variáveis não normalizadas & Variáveis normalizadas \\
\hline FOU & $\phi_{f}=\phi_{U}$ & $\hat{\phi}_{f}=\hat{\phi}_{U}$ \\
CDS & $\phi_{f}=\frac{1}{2}\left(\phi_{D}+\phi_{U}\right)$ & $\hat{\phi}_{f}=\frac{3}{4}+\frac{1}{2}\left(\hat{\phi}_{U}-\frac{1}{2}\right)$ \\
QUICK & $\phi_{f}=\frac{1}{2}\left(\phi_{D}+\phi_{U}\right)-\frac{1}{8}\left(\phi_{D}-2 \phi_{U}+\phi_{R}\right)$ & $\hat{\phi}_{f}=\frac{3}{4}+\frac{3}{4}\left(\hat{\phi}_{U}-\frac{1}{2}\right)$ \\
SOU & $\phi_{f}=\frac{3}{2} \phi_{U}-\frac{1}{2} \phi_{D}$ & $\hat{\phi}_{f}=\frac{3}{2} \hat{\phi}_{U}$ \\
\hline \hline
\end{tabular}

Usando o desenvolvimento em série de Taylor, pode-se mostrar (ver Leonard (1988a)) que para qualquer esquema (linear ou não linear) em variáveis normalizadas valem as seguintes propriedades:

- um esquema em variáveis normalizadas que passa pelos pontos $\mathrm{O}(0,0)$ e $\mathrm{P}(1,1)$ é limitado (condição necessária);

- um esquema em variáveis normalizadas que passa pelo ponto $Q(1 / 2,3 / 4)$ alcança segunda ordem de precisão (condição necessária e suficiente);

- um esquema em variáveis normalizadas que passa pelo ponto $\mathrm{Q}(1 / 2,3 / 4)$ e tem inclinação $3 / 4$ nesse ponto, alcança terceira ordem de precisão (condição necessária e suficiente).

A ideia de se construir um esquema limitado que atinja (ao menos) segunda ordem e produza soluções suaves limitadas é que sua característica em variáveis normalizadas satisfaça as condições a 
seguir:

$$
\begin{array}{r}
O(0,0) ; \\
P(1,1) ; \\
Q(1 / 2,3 / 4) ;
\end{array}
$$

e para terceira ordem a característica deve passar por esses pontos com derivada $3 / 4 \mathrm{em} Q$.

\subsubsection{Critérios de limitação}

Soluções numéricas limitadas e sem oscilações não físicas são de suma importância para o transporte de propriedades físicas (Tao et al., 2005). Com o objetivo de obter soluções limitadas faz-se necessário que a variação da variável convectada $\phi$ nas faces $f$ e $g$ (ver Fig. 2.3) esteja localmente limitada entre os valores vizinhos. Por exemplo, para a face $f$ deve-se ter

$$
\phi_{U} \leq \phi_{f} \leq \phi_{D}
$$

Para alcançar tal objetivo, Gaskell e Lau (1988) propuseram o critério convection boundedness criterion (CBC) para limitação da característica em variáveis normalizadas, isto é

$$
\begin{cases}\hat{\phi}_{f} \in\left[\hat{\phi}_{U}, 1\right], & \text { se } \quad \hat{\phi}_{U} \in[0,1] ; \\ \hat{\phi}_{f}=1, & \text { se } \quad \hat{\phi}_{U}=1 ; \\ \hat{\phi}_{f}=0, & \text { se } \quad \hat{\phi}_{U}=0 ; \\ \hat{\phi}_{f}=\hat{\phi}_{U}, & \text { se } \quad \hat{\phi}_{U} \notin[0,1] .\end{cases}
$$

A Fig. 2.5 ilustra no diagrama em variáveis normalizadas a região de limitação CBC.

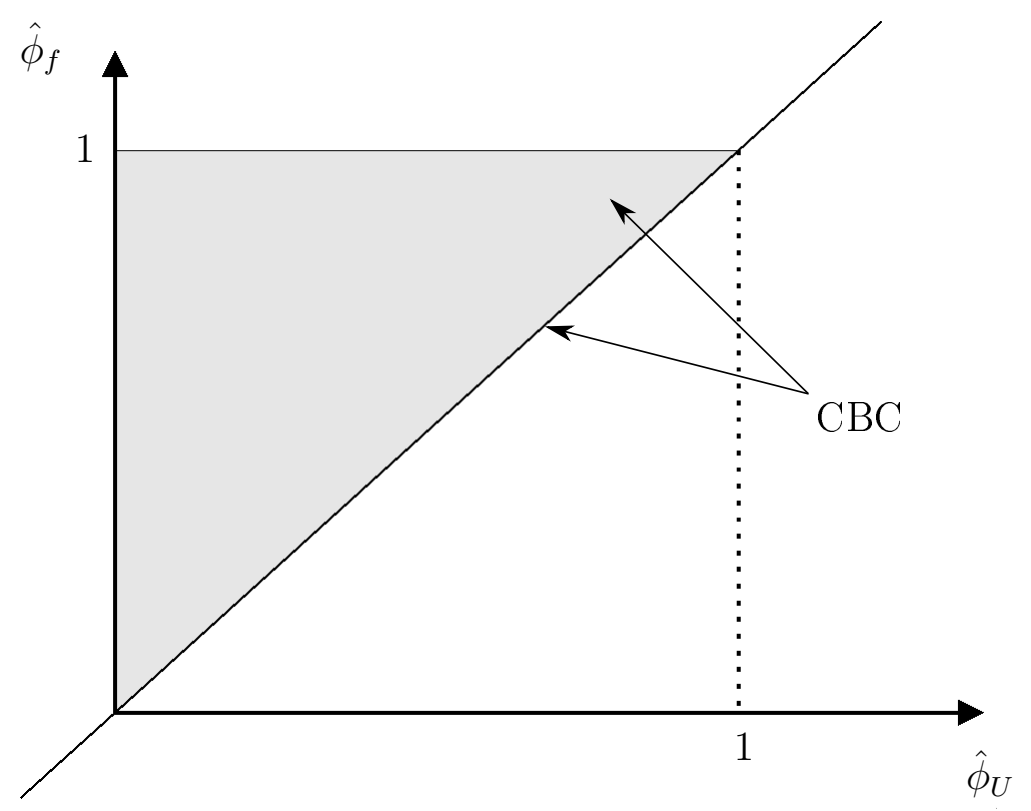

Figura 2.5: Diagrama de variáveis normalizadas: Região CBC. 
Portanto, obtém-se soluções limitadas se o esquema dado por (2.7) está inteiramente contido na região CBC. Vale observar que desde há muito o critério CBC tem sido aceito como uma condição necessária e suficiente para derivar esquemas limitados (Gaskell e Lau, 1988, Darvish, 1993, Choi et al., 1995, Waterson e Deconinck, 2007, Kemm, 2010, Ferreira et al., 2012, Lima et al., 2012). Todavia, nos trabalhos de Tao (2000), Yu et al. (2001) e Wei et al. (2006) os autores provaram que a condição de limitação CBC é apenas suficiente; e, então, eles propuseram novas restrições para limitação chamada extended $\mathrm{CBC}$ (ECBC) e ilustrada na Fig. 2.6. Os resultados numéricos apresentados por esses autores mostraram ser satisfatórios, tanto em limitação como acurácia.

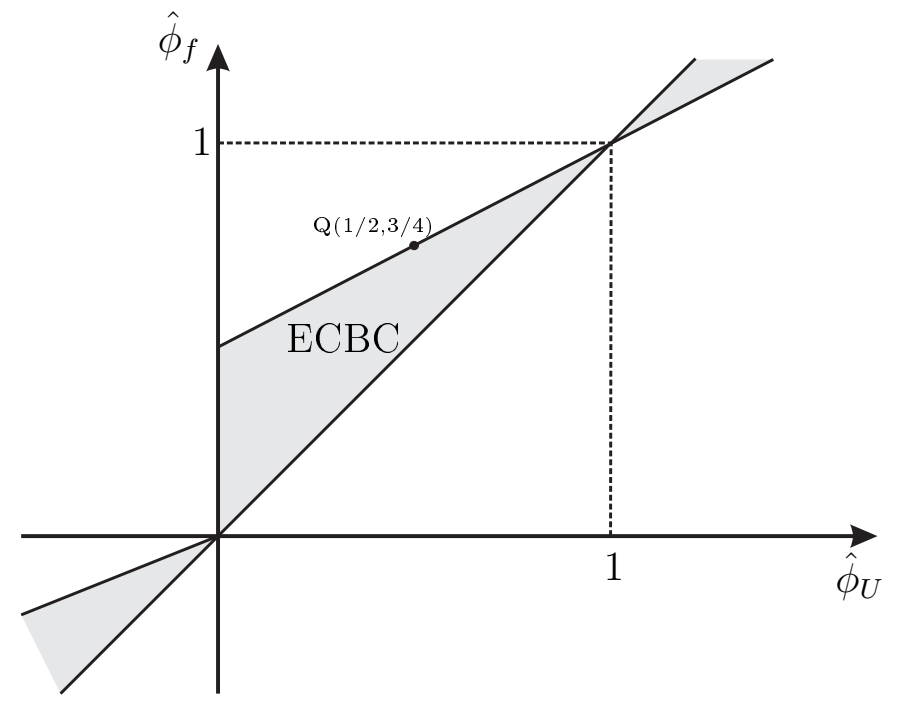

Figura 2.6: Diagrama de variáveis normalizadas: Região ECBC.

Outro critério de limitação importante, e que garante monotonicidade da solução, é o total variation diminishing (TVD) de Harten et al. (1976) (ver também Sweby e Baines (1981), Harten (1983)); inicialmente define-se a variação total da solução numérica $\phi$ no tempo $n+1$ por

$$
T V\left(\phi^{n+1}\right)=\sum_{k}\left|\phi_{k+1}^{n+1}-\phi_{k}^{n+1}\right|, \quad k \in \mathbb{N}
$$

Diz-se então que um esquema de convecção é TVD se ele produz solução numérica que satisfaz

$$
T V\left(\phi^{n+1}\right) \leq T V\left(\phi^{n}\right)
$$

Os métodos construídos satisfazendo o conceito de limitação TVD caracterizam-se por serem conservativos, limitados e impedem a formação de oscilações não físicas (espúrias) nas soluções numéricas (Harten, 1983). No contexto de variáveis normalizadas, as condições para um esquema ser TVD são (ver Harten (1983))

$$
\left\{\begin{array}{lll}
\hat{\phi}_{f} \in\left[\hat{\phi}_{U}, 2 \hat{\phi}_{U}\right] & \text { e } \quad \hat{\phi}_{f} \leq 1, & \hat{\phi}_{U} \in[0,1], \\
\hat{\phi}_{f}=\hat{\phi}_{U}, & \hat{\phi}_{U} \notin[0,1] .
\end{array}\right.
$$

A Fig. 2.7 apresenta a região TVD no diagrama de variáveis normalizadas.

A seguir, apresentam-se exemplos de esquemas convectivos upwind convencionais em variáveis nor- 


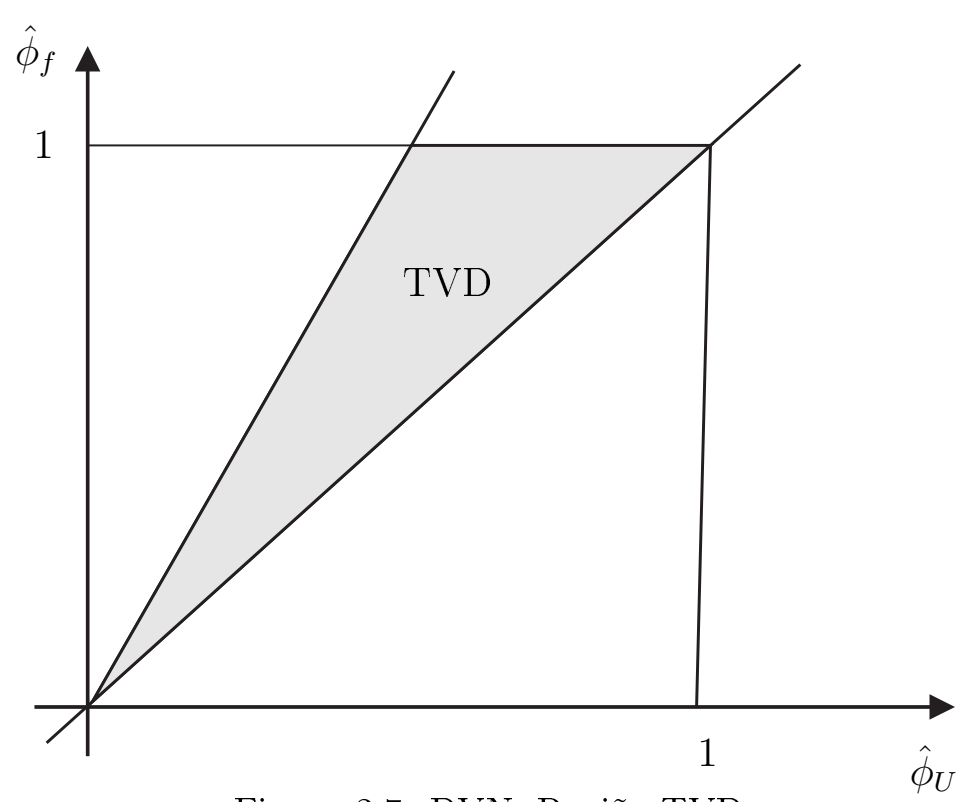

Figura 2.7: DVN: Região TVD.

malizadas, alguns deles utilizados neste trabalho para comparação.

- ADBQUICKEST de Ferreira et al. (2009): Este esquema é uma versão limitada do esquema QUICKEST de Leonard (1979) e contempla o número de $C F L, \theta=a \delta_{t} / \delta_{x}$ (sendo $a$ uma velocidade de convecção), em sua formulação. O ADBQUICKEST tem sido usado na literatura para a simulação de sistemas hiperbólicos complexos de leis de conservação (ver, por exemplo, Candezano et al. (2010a,b), Le (2011), Ferreira et al. (2012)) e problemas de escoamento incompressíveis de fluidos newtonianos/não newtonianos nos regimes laminar e turbulento (Ferreira et al., 2009, Kurokawa, 2009). O ADBQUICKEST tem sido um esquema padrão no código Freeflow e sua formulação é como segue:

$$
\hat{\phi}_{f}= \begin{cases}(2-\theta) \hat{\phi}_{U}, & 0<\hat{\phi}_{U}<a_{1}, \\ \hat{\phi}_{U}+\frac{1}{2}(1-|\theta|)\left(1-\hat{\phi}_{U}\right)-\frac{1}{6}\left(1-\theta^{2}\right)\left(1-2 \hat{\phi}_{U}\right), & a_{1}<f u<b_{1}, \\ 1-|\theta|+|\theta| \hat{\phi}_{U}, & b_{1}<\hat{\phi}_{U}<1, \\ \hat{\phi}_{U}, & \text { caso contrário, }\end{cases}
$$

onde $a_{1}=\frac{2-3|\theta|+\theta^{2}}{7-6|\theta|-3 \theta+2 \theta^{2}} \quad$ e $\quad b_{1}=\frac{-4+6|\theta|-3 \theta+\theta^{2}}{-5+6 \theta-3|\theta|+2 \theta^{2}}$.

- ARORA-ROE de Arora e Roe (1997): Este é um esquema derivado dos trabalhos de Roe e Baines (1982) e Roe (1985) e faz parte da família dos esquemas dependentes do número de CFL. 
A sua formulação é como segue:

$$
\hat{\phi}_{f}= \begin{cases}\hat{\phi}_{U} \frac{|\theta|+(1-\theta)}{|\theta|}, & 0<\hat{\phi}_{U}<a_{2}, \\ \hat{\phi}_{U}+\frac{1}{6}\left[2-\hat{\phi}_{U}+|\theta|\left(2 \hat{\phi}_{U}-1\right)\right](1-\theta), & a_{2}<\hat{\phi}_{U}<b_{2}, \\ \hat{\phi}_{U}+\frac{1-\theta}{1-|\theta|}\left(1-\hat{\phi}_{U}\right), & b_{2}<\hat{\phi}_{U}<1, \\ \hat{\phi}_{U}, & \text { caso contrário, }\end{cases}
$$

onde $a_{2}=\frac{2|\theta|-|\theta|^{2}}{6+|\theta|-2|\theta|^{2}} \quad$ e $\quad b_{2}=\frac{6-(2-|\theta|)(1-|\theta|)}{6+(1-|\theta|)(2|\theta|-1)}$.

- CUBISTA de Alves et al. (2003): Este é um esquema desenvolvido para melhorar as propriedades de convergência em escoamentos transientes. A sua expressão é como segue:

$$
\hat{\phi}_{f}= \begin{cases}\frac{7}{4} \hat{\phi}_{U}, & 0<\hat{\phi}_{U}<3 / 8 \\ \frac{3}{4} \hat{\phi}_{U}+\frac{3}{8}, & 3 / 8 \leq \hat{\phi}_{U} \leq 3 / 4 \\ \frac{1}{4} \hat{\phi}_{U}+\frac{3}{4}, & 3 / 4<\hat{\phi}_{U}<1 \\ \hat{\phi}_{U}, & \hat{\phi}_{U} \notin(0,1)\end{cases}
$$

- TOPUS de Ferreira et al. (2012): Este esquema é o primeiro da família dos esquemas upwind polinomiais desenvolvido no LMACC do ICMC-USP. TOPUS depende de um parâmetro livre $\alpha$, atingindo seu melhor desempenho em $\alpha=2$. A sua formulação é como segue:

$$
\hat{\phi}_{f}= \begin{cases}\alpha \hat{\phi}_{U}^{4}+(-2 \alpha+1) \hat{\phi}_{U}^{3}+\left(\frac{5 \alpha-10}{4}\right) \hat{\phi}_{U}^{2}+\left(\frac{-\alpha+10}{4}\right) \hat{\phi}_{U}, & \hat{\phi}_{U} \in[0,1] ; \\ \hat{\phi}_{U}, & \hat{\phi}_{U} \notin[0,1] .\end{cases}
$$

- SUPERBEE de Roe (1986): Este é um esquema de segunda ordem de precisão local, popularmente usado em problemas compressíveis, e tem sido um esquema padrão no código MFiX (2011) para a simulação computacional de escoamentos de duas fases gás-sólido. A sua formulação é como segue:

$$
\hat{\phi}_{f}= \begin{cases}2 \hat{\phi}_{U}, & 0 \leq \hat{\phi}_{U}<\frac{1}{3} \\ \frac{1}{2}\left(1+\hat{\phi}_{U}\right), & \frac{1}{3} \leq \hat{\phi}_{U}<\frac{1}{2} \\ \frac{3}{2} \hat{\phi}_{U}, & \frac{1}{2} \leq \hat{\phi}_{U}<\frac{2}{3} \\ 1, & \frac{2}{3} \leq \hat{\phi}_{U} \leq 1 \\ \hat{\phi}_{U}, & \hat{\phi}_{U} \notin[0,1]\end{cases}
$$

- MC de van Leer (1977): Este é um esquema de segunda ordem de precisão local e tem sido o 
esquema padrão no código CLAWPACK. Em resumo, a formulação do MC é como segue:

$$
\hat{\phi}_{f}= \begin{cases}2 \hat{\phi}_{U}, & 0 \leq \hat{\phi}_{U}<\frac{1}{4} \\ \hat{\phi}_{U}+\frac{1}{4}, & \frac{1}{4} \leq \hat{\phi}_{U}<\frac{3}{4} \\ 1, & \frac{3}{4} \leq \hat{\phi}_{U} \leq 1 \\ \hat{\phi}_{U}, & \hat{\phi}_{U} \notin[0,1]\end{cases}
$$

\subsubsection{Limitadores de fluxo}

A ideia básica dos limitadores de fluxo (LF) é limitar esquemas não monotônicos. Tradicionalmente, os limitadores de fluxo são embutidos em esquemas numéricos de alta resolução, tais como o esquema MUSCL de van Leer (1979), para evitar o aparecimento de oscilações não físicas na solução numérica. O conceito de limitador de fluxo foi introduzido por van Leer $(1973,1974)$ e Boris e Book $(1973,1976)$ numa série de trabalhos sobre os esquemas upwind de segunda ordem que não produzem oscilações numéricas (ver detalhes em Hirsch (2007)).

Com o intuito de precisar melhor o conceito de limitador de fluxo, considera-se o transporte unidimensional de um escalar $u$ com velocidade $a>0$ modelado pela equação linear $u_{t}+a u_{x}=0$. Soluções numéricas para essa equação podem ser obtidas via o esquema (não TVD) de Lax e Wendroff (1960)-LW (ou uma discretização de segunda ordem, ou ainda o esquema upwind de segunda ordem de Warming e Beam (1976)-WB). Em particular, o método de LW para essa equação linear é dado por

$$
u_{i}^{n+1}=u_{i}^{n}-\theta\left(u_{i+1}^{n}-u_{i-1}^{n}\right)+\frac{\theta^{2}}{2}\left(u_{i+1}^{n}-2 u_{i}^{n}+u_{i-1}^{n}\right),
$$

onde a discretização foi feita tomando-se como base o ponto de malha $\left(i \delta_{x}, n \delta_{t}\right)$ e usando-se diferença para frente no tempo (método de Euler) e central no espaço. A Fig. 2.8 mostra a solução numérica via o método (2.22) e solução analítica para o transporte de uma onda quadrada via a equação advecção (2.2) com $u=1$. Vê-se claramente por essa figura que o esquema de LW gera soluções numéricas não limitadas.

Este esquema pode ser separado em uma parte de primeira ordem (FOU) mais uma parte que corresponde a termos não monotônicos de correção, isto é

$$
u_{i}^{n+1}=\underbrace{u_{i}^{n}-\theta\left(u_{i}^{n}-u_{i-1}^{n}\right)}_{\text {FOU }}+\underbrace{\frac{\theta}{2}(1-\theta)\left(u_{i}^{n}-u_{i-1}^{n}\right)-\frac{\theta}{2}(1-\theta)\left(u_{i+1}^{n}-u_{i}^{n}\right)}_{\text {termos não monótonos }} .
$$

Para tornar o esquema (2.23) limitado (TVD), multiplica-se a sua segunda parte por uma função $\Psi=\Psi(r)$ (que é o limitador de fluxo), onde $r$ é um sensor de suavidade que detecta gradientes elevados (pontos extremos, descontinuidades ou choques) e definido como a razão dos gradientes consecutivos numa face da célula computacional. No caso da Fig. 2.3, esse detector torna-se

$$
r_{i}=\left(\frac{\partial u}{\partial x}\right)_{g} /\left(\frac{\partial u}{\partial x}\right)_{f} \approx \frac{u_{i}-u_{i-1}}{u_{i+1}-u_{i}}=\frac{u_{U}-u_{R}}{u_{D}-u_{U}}
$$




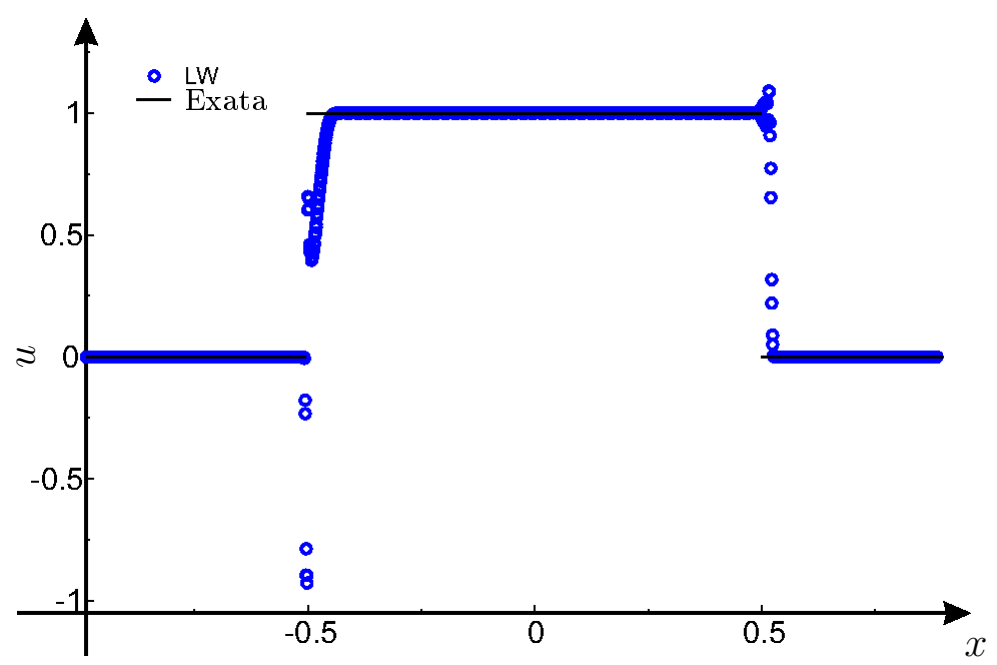

Figura 2.8: Comparação entra as soluções numéricas obtidas com o esquema Lax-Wendroff e analítica do problema de advecção. $N=2000$ e $C F L=0.3$.

Multiplicando-se o primeiro termo não monótono na Eq. (2.23) por $\Psi\left(r_{i-1}\right)$ e o segundo por $\Psi\left(r_{i}\right)$, a Eq. (2.23) pode ser rearranjada para obter

$$
u_{i}^{n+1}=u_{i}^{n}-\theta\left(u_{i}^{n}-u_{i-1}^{n}\right)\left\{1+\frac{1}{2}(1-\theta)\left[\frac{\Psi\left(r_{i}\right)}{r_{i}}-\Psi\left(r_{i-1}\right)\right]\right\} .
$$

Segundo Harten (1983), o esquema (2.25) é limitado e monotônico se o termo $\{\ldots\}$ for positivo, acarretando a desigualdade

$$
\Psi\left(r_{i-1}\right)-\frac{\Psi\left(r_{i}\right)}{r_{i}} \leq \frac{2}{1-\theta} .
$$

Os detalhes do desenvolvimento do limitador de fluxo pode ser encontrado, por exemplo, em Hirsch (2007).

O método de LW dado em (2.25) é uma motivação para o desenvolvimento de esquemas upwind de alta ordem limitados. De maneira geral, um esquema convectivo tipo upwind pode ser desenvolvido, tomando-se como base a interpolação para $\phi_{f}$ na Fig. (2.3), por meio da aproximação

$$
\phi_{f}=\phi_{U}+B\left[\left.\left(\frac{\partial \phi}{\partial x}\right)\right|_{f},\left.\left(\frac{\partial \phi}{\partial x}\right)\right|_{g}, \delta x\right],
$$

onde a função $B$ representa um termo de correção anti-difusivo. Neste trabalho, toma-se $B$ como

$$
B=\left.\frac{1}{2} \delta x\left(\frac{\partial \phi}{\partial x}\right)\right|_{f}
$$

O esquema convectivo upwind (2.27) pode ser então aproximado por

$$
\phi_{f} \approx \phi_{U}+\left.\frac{1}{2} \delta x\left(\frac{\partial \phi}{\partial x}\right)\right|_{f} \approx \phi_{U}+\frac{1}{2}\left(\phi_{D}-\phi_{U}\right)
$$

o qual é propenso à formação de oscilações não físicas (similar ao esquema de LW (2.22)). Para evitar 
essas oscilações, introduz-se um limitador de fluxo $\Psi$ na formulação (2.29) para obter

$$
\phi_{f} \approx \phi_{U}+\frac{1}{2} \Psi\left(r_{f}\right)\left(\phi_{D}-\phi_{U}\right)
$$

o qual em variáveis normalizadas é expresso como

$$
\hat{\phi}_{f}=\hat{\phi}_{U}+\frac{1}{2} \Psi\left(r_{f}\right)\left(1-\hat{\phi}_{U}\right)
$$

com o sensor $r_{f}$ dado por

$$
r_{f}=\frac{\hat{\phi}_{U}}{1-\hat{\phi}_{U}}
$$

Como comentado anteriormente, o parâmetro $r_{f}$ funciona como um detector de suavidade da solução, e com base em seus valores várias regiões de interesse podem ser identificadas no plano $r_{f} \perp \Psi\left(r_{f}\right)$. A reta $r_{f}=0$, como mostrado na Figura 2.9, separa regiões de oscilações ou extremos $\left(r_{f}<0\right)$ de regiões de soluções monotônicas $\left(r_{f}>0\right)$. Dentro dessas últimas, $r_{f} \approx 1$ corresponde a regiões de soluções suaves $\left(r_{f}=1\right.$ - variação linear). Regiões de altas curvaturas podem ser identificadas para $r_{f} \leq 0$ e $r_{f}<<1$ (curvatura negativa) ou $r_{f}>>1$ (curvatura positiva).

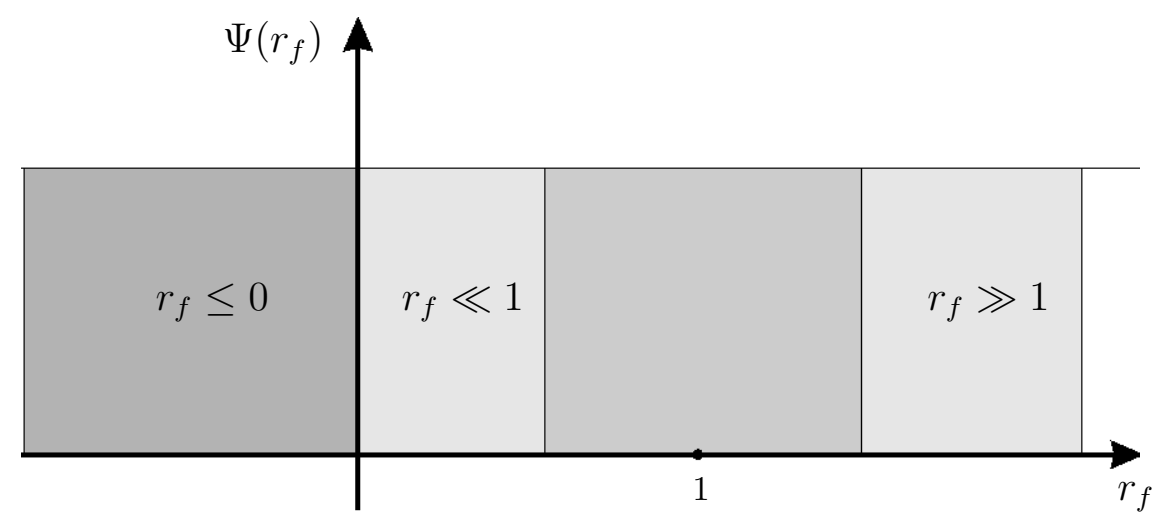

Figura 2.9: Plano $\Psi\left(r_{f}\right) \perp r_{f}$ mostrando a região de extremos $\left(r_{f} \leq 0\right)$, a região de monotonicidade (vizinhança de $r_{f}=1$ ) e as regiões de alta curvatura $\left(r_{f}>>1\right.$ e $r_{f}<<1$ ).

Pode-se mostrar (veja, por exemplo, Sweby (1984)) que se o limitador de fluxo $\Psi\left(r_{f}\right)$ satisfaz as propriedades

- $\Psi\left(r_{f}\right)=0, r \leq 0$;

- $0 \leq \Psi\left(r_{f}\right) \leq 2 r_{f}$;

- $\Psi\left(r_{f}\right) \leq 2$,

então o esquema numérico (2.31) é livre de oscilações e, neste caso, diz-se que o limitador de fluxo é TVD. Alguns exemplos de limitadores de fluxo (incluindo FOU, LW e WB) no diagrama $r_{f} \perp \Psi\left(r_{f}\right)$ são apresentados na Fig. 2.10. Em particular, esquemas monotônicos de segunda ordem devem satisfazer à seguinte desigualdade

$$
0 \leq \Psi\left(r_{f}\right) \leq \min \left(2 r_{f}, 2\right)
$$




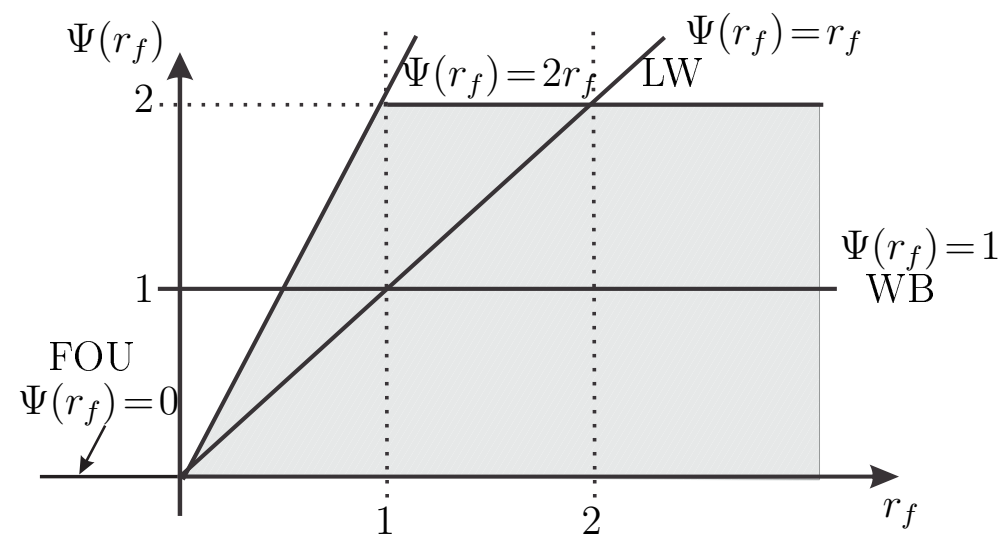

Figura 2.10: Região TVD de Sweby.

A seguir está mostrado que qualquer limitador de fluxo $\Psi$ que satisfaça $\Psi(1)=1$ (condição necessária (Waterson e Deconinck, 2007) ) e fazendo com que (2.30) seja de segunda ordem de precisão em malhas uniformes, deve satisfazer a seguinte condição de equivalência:

$$
\left.\frac{d \Psi}{d r_{f}}\right|_{r_{f}=1}=\frac{1}{4} \Leftrightarrow \text { o esquema (2.30) tem terceira ordem de precisão local. }
$$

De fato, pois:

$(\Leftarrow)$ Levando os desenvolvimentos, em série de taylor em torno do ponto $(i+1 / 2, j)$, de $\phi_{(i-1, j)}=$ $\phi_{([i+1 / 2]-3 / 2, j)}, \phi_{(i, j)}=\phi_{([i+1 / 2]-1 / 2, j)}$ e $\phi_{(i+1, j)}=\phi_{([i+1 / 2]+1 / 2, j)}$, mais $\psi\left(r_{f}\right) \approx \psi(1)+\left.\left(r_{f}-1\right) \frac{d \psi}{d r_{f}}\right|_{r_{f}=1}$, na expressão $(2.30)$, resulta $\left.\frac{d \psi}{d r_{f}}\right|_{r_{f}=1}=\frac{1}{4}$.

$(\Rightarrow)$ A derivada da expressão $(2.31)$ em relação $\hat{\phi}_{(i, j)}$ dá

$$
\begin{gathered}
\frac{d \hat{\phi}_{(i+1 / 2, j)}}{d \hat{\phi}_{(i, j)}}=1+\frac{1}{2} \frac{d}{d \hat{\phi}_{(i, j)}}\left[\Psi\left(r_{f(i+1 / 2, j)}\right)\left(1-\hat{\phi}_{(i, j)}\right)\right] \\
=1+\frac{1}{2}\left[\frac{d \Psi}{d r_{f(i+1 / 2, j)}} \cdot \frac{d r_{f(i+1 / 2, j)}}{d \hat{\phi}_{(i, j)}} \cdot\left(1-\hat{\phi}_{(i, j)}\right)-\Psi\left(r_{f(i+1 / 2, j)}\right)\right] \\
=1+\frac{1}{2}\left[\frac{d \Psi}{d r_{f(i+1 / 2, j)}} \cdot \frac{1}{\left(1-\hat{\phi}_{(i, j)}\right)^{2}} \cdot\left(1-\hat{\phi}_{(i, j)}\right)-\Psi\left(r_{f(i+1 / 2, j)}\right)\right] .
\end{gathered}
$$

Avaliando a expressão $(2.35)$ em $\hat{\phi}_{(i, j)}=\frac{1}{2}$ (o que equivale a $r_{f(i+1 / 2, j)}=1$ ) obtém-se

$$
\frac{d \hat{\phi}_{(i+1 / 2, j)}}{d \hat{\phi}_{(i, j)}}=\frac{3}{4} .
$$

Essa é a condição de Leonard (1988a) para esquemas upwind alcançarem terceira ordem de precisão.

Utilizando-se da ideia de monotonicidade, Sweby (1984) definiu o princípio de monotonicidade para limitadores de fluxo (condição suficiente), em que

$$
\Psi^{\prime}\left(r_{f}\right)=2 \quad \text { se } \quad r_{f} \rightarrow 0 .
$$


A Fig. 2.11 descreve a região de monotonicidade de Sweby para esquemas de segunda ordem de precisão local.

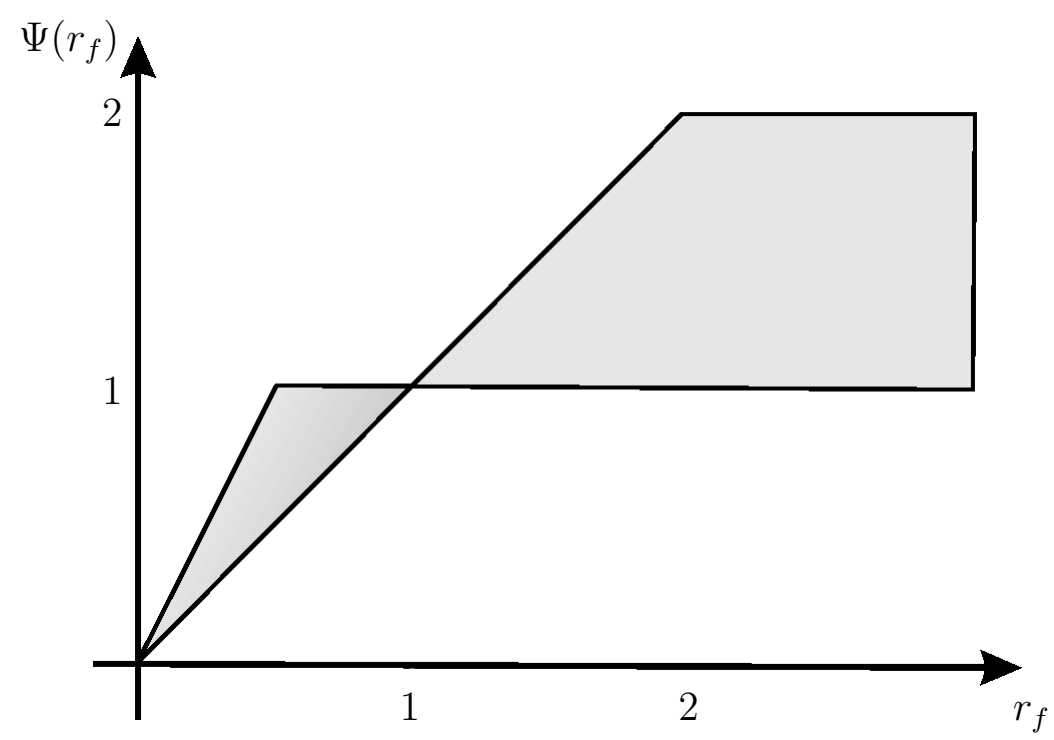

Figura 2.11: Região TVD de segunda ordem nas variáveis $(\Psi, r)$.

É interessante observar que a região TVD apresentada na Fig. 2.11 está inteiramente contida na região (mais geral) de positividade de Spekreijse (1986) (com $\mathrm{M} \geq 2$ e $\alpha \leq 0$ ). Na sequência, apresentam-se alguns limitadores de fluxo de esquemas upwind consagrados na literatura.

- ADBQUICKEST, Ferreira et al. (2009):

$$
\Psi(r)=\max \left\{0, \min \left[2 r(1-\theta), \frac{2+\theta^{2}-3|\theta|+\left(1-\theta^{2}\right) r}{3}, 2(1-\theta)\right]\right\}
$$

- ARORA-ROE, Arora e Roe (1997):

$$
\Psi(r)=\max \left\{0, \min \left[\frac{2}{|\theta|} r, 1+\frac{1+|\theta|}{3}(r-1), \frac{2}{1-|\theta|}\right]\right\} ;
$$

- CUBISTA, Alves et al. (2003):

$$
\Psi(r)=\max \left[0, \min \left\{\frac{3}{2} r, \frac{3}{4} r+\frac{1}{4}, \frac{3}{2}\right\}\right]
$$

- TOPUS, Ferreira et al. (2012):

$$
\Psi(r)=\frac{0.5(|r|+r)\left[(-0.5 \alpha+1) r^{2}+(\alpha+4) r+(-0.5 \alpha+3)\right]}{(1+r)^{4}}, \quad \alpha=2 ;
$$

- SUPERBEE, Roe (1986):

$$
\Psi(r)=\max [0, \min (2 r, 1), \min (r, 2)]
$$


- MC, van Leer (1977):

$$
\Psi(r)=\max [0, \min (2 r, 0.5(1+r), 2)]
$$

- MINMOD, Roe (1986):

$$
\Psi(r)=\max [0, \min (1, r)]
$$

\subsection{Desenvolvimento do novo esquema TDPUS-C3}

Nesta secção será apresentado um novo esquema convectivo do tipo upwind de alta ordem para o cálculo dos fluxos numéricos nas interfaces $f$ e $g$ das células computacionais (ver Fig.2.3). Mais uma vez, aqui, considera-se somente a face $f$.

A nova metodologia desenvolvida neste trabalho trata-se de um esquema upwind polinomial de grau dez no intervalo [0,1] e de classe C3, doravante chamado Tenth Degree Polynomial Upwind Scheme (TDPUS-C3), e fora deste coincide intervalo, por simplicidade, é implementado o esquema upwind de primeira ordem- FOU $\left(\hat{\phi}_{f}=\hat{\phi}_{U}\right)$. Este polinômio em variáveis normalizadas é expresso como

$$
\hat{\phi}_{f}\left(\hat{\phi}_{U}\right)=\sum_{i=0}^{10} \alpha_{i} \hat{\phi}_{U}^{i},
$$

onde os coeficientes são determinados impondo-se as condições de Leonard (2.8), (2.9) e (2.10) mais as condições (para suavidade)

$$
\begin{aligned}
\hat{\phi}_{f}^{\prime}(1 / 2) & =3 / 4 ; \\
\hat{\phi}_{f}^{\prime}(0) & =1 ; \\
\hat{\phi}_{f}^{\prime}(1) & =1 ; \\
\hat{\phi}_{f}^{\prime \prime}(0) & =0 ; \\
\hat{\phi}_{f}^{\prime \prime}(1) & =0 ; \\
\hat{\phi}_{f}^{\prime \prime \prime}(0) & =0 ; \\
\hat{\phi}_{f}^{\prime \prime \prime}(1) & =0 .
\end{aligned}
$$

A condição (2.45) é imposta para que o esquema atinja terceira ordem de precisão local (ver Leonard (1988a)). As condições (2.46) e (2.47) são impostas para evitar problemas de convergência em malhas grosseiras (ver Lin e Chieng (1991)). As condições (2.48) e (2.49) servem para se obter suavidade (ver Zijlema (1996) e Corrêa et al. (2010)). As últimas duas condições tem como propósito impor que a curvatura varia lentamente (poucas mudanças bruscas).

As condições (2.8)-(2.10) e (2.45)-(2.51), mais a imposição de que $\alpha_{4}=\beta \in \mathbb{R}$ seja um parâmetro livre, constituem um sistema de equações lineares nos coeficientes $\alpha_{i}, i=0, \ldots, 10$, da forma 


$$
\left[\begin{array}{cccccccccc}
1 & 0 & 0 & 0 & 0 & 0 & 0 & 0 & 0 & 0 \\
0 & 1 & 0 & 0 & 0 & 0 & 0 & 0 & 0 & 0 \\
0 & 0 & 1 & 0 & 0 & 0 & 0 & 0 & 0 & 0 \\
0 & 0 & 0 & 1 & 0 & 0 & 0 & 0 & 0 & 0 \\
1 & 1 & 1 & 1 & 1 & 1 & 1 & 1 & 1 & 1 \\
1024 & 512 & 256 & 128 & 32 & 16 & 8 & 4 & 2 & 1 \\
0 & \frac{256}{5} & \frac{256}{5} & \frac{192}{5} & 16 & \frac{48}{5} & \frac{28}{5} & \frac{16}{5} & \frac{9}{5} & 1 \\
0 & 1 & 2 & 3 & 5 & 6 & 7 & 8 & 9 & 10 \\
0 & 0 & 2 & 6 & 20 & 30 & 42 & 56 & 72 & 90 \\
0 & 0 & 0 & 6 & 60 & 120 & 210 & 336 & 504 & 720
\end{array}\right]\left[\begin{array}{c}
\alpha_{0} \\
\alpha_{1} \\
\alpha_{2} \\
\alpha_{3} \\
\alpha_{5} \\
\alpha_{6} \\
\alpha_{7} \\
\alpha_{8} \\
\alpha_{9} \\
\alpha_{10}
\end{array}\right]=\left[\begin{array}{c}
0 \\
1 \\
0 \\
0 \\
1-\beta \\
768-64 \beta \\
\frac{192}{5}-\frac{128}{5} \beta \\
1-4 \beta \\
-12 \beta \\
-24 \beta
\end{array}\right]
$$

cuja solução obtida pelo software wxMaxima (2011) é

$$
\boldsymbol{\alpha}=[0,1,0,0,320-8 \beta, 26 \beta-1664,3456-44,41 \beta-3584,1856-20 \beta, 4 \beta-384]^{T} .
$$

Substituindo os coeficientes (2.53) em (2.44) obtém-se a expressão para o esquema TDPUS-C3

$$
\hat{\phi}_{f}= \begin{cases}(4 \beta-384) \hat{\phi}_{U}^{10}+(1856-20 \beta) \hat{\phi}_{U}^{9}+(41 \beta-3584) \hat{\phi}_{U}^{8}+(3456 & \\ -44 \beta) \hat{\phi}_{U}^{7}+(26 \beta-1664) \hat{\phi}_{U}^{6}+(320-8 \beta) \hat{\phi}_{U}^{5}+\beta \hat{\phi}_{U}^{4}+\hat{\phi}_{U}, & \hat{\phi}_{U} \in[0,1] ; \\ \hat{\phi}_{U}, & \hat{\phi}_{U} \notin[0,1],\end{cases}
$$

que em variáveis não normalizadas (para implementação computacional) torna-se

$$
\phi_{f}= \begin{cases}\phi_{R}+\left(\phi_{D}-\phi_{R}\right) *\left(\hat{\phi}_{U}+\hat{\phi}_{U}^{4}\left(\alpha_{4}+\hat{\phi}_{U}\left(\alpha_{5}+\hat{\phi}_{U}\left(\alpha_{6}+\right.\right.\right.\right. & \\ \left.\left.\left.\hat{\phi}_{U}\left(\alpha_{7}+\hat{\phi}_{U}\left(\alpha_{8}+\hat{\phi}_{U}\left(\alpha_{9}+\hat{\phi}_{U} \alpha_{10}\right)\right)\right)\right)\right)\right), & \hat{\phi}_{U} \in[0,1] ; \\ \phi_{U}, & \hat{\phi}_{U} \notin[0,1],\end{cases}
$$

onde

$$
\begin{gathered}
\alpha_{10}=4 \beta-384, \alpha_{9}=1856-20 \beta, \alpha_{8}=41 \beta-3584, \alpha_{7}=3456-44 \beta, \\
\alpha_{6}=26 \beta-1664, \alpha_{5}=320-8 \beta, \text { e } \alpha_{4}=\beta .
\end{gathered}
$$

O esquema TDPUS-C3 assim proposto torna-se TVD dependendo do valor do parâmetro $\beta$. A Tab. 2.2 apresenta alguns valores selecionados do intervalo $[-200,200]$ com o tamanho do passo 0.25 (isto é, $\beta=64,226,566,559$ e 567.25), os quais têm fornecido bons resultados em advecção de escalares. A Fig. 2.12 mostra que o esquema TDPUS-C3, para esses valores do parâmetro $\beta$, satisfaz a propriedade de limitação TVD. 
Tabela 2.2: Diferentes valores do parâmetro $\beta$ para o esquema TDPUS-C3

\begin{tabular}{ccccc}
\hline \hline \multicolumn{5}{c}{$\beta$} \\
\hline 64 & 85 & 110 & 123 & 140 \\
168 & 185 & 202 & 231 & 243 \\
266 & 283 & 300 & 326 & 347 \\
382 & 400 & 425 & 440 & 460 \\
478 & 502 & 526 & 559 & 567.25
\end{tabular}

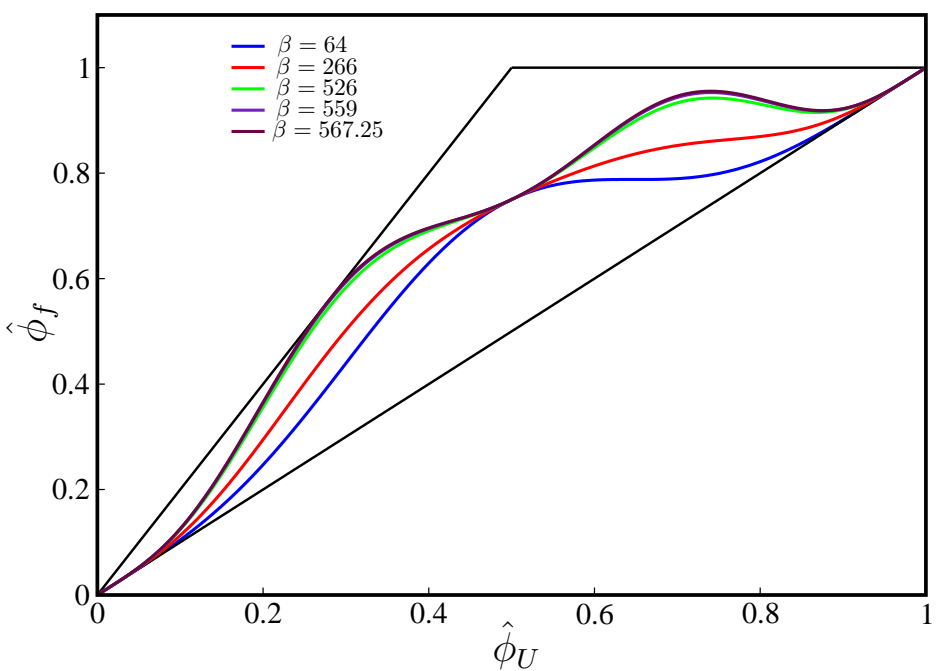

Figura 2.12: Região TVD para o esquema TDPUS-C3 em variáveis normalizadas.

O limitador de fluxo correspondente para o esquema TDPUS-C3 é obtido fazendo uso das Eqs. (2.31), (2.32) e (2.54), cujo resultado é

$$
\Psi(r)= \begin{cases}\frac{(2 \beta-128) r^{6}+(640-4 \beta) r^{5}+2 \beta r^{4}}{(1+r)^{9}}, & r \geq 0 ; \\ 0, & r<0 .\end{cases}
$$

Vê-se por essa expressão que o limitador de fluxo do esquema TDPUS-C3 possui as propriedades $\Psi(1)=1$ e $\Psi^{\prime}(1)=\frac{1}{4}$, alcançando portanto terceira ordem de precisão local. A Fig. 2.13 apresenta o esquema TDPUS-C3 com os parâmetros livres destacados na Tab. 2.2, na região TVD de Sweby.

Na notação mais usual (ver, por exemplo, Waterson e Deconinck (2007)), o correspondente limitador de fluxo de TDPUS-C3 pode ser escrito como

$$
\Psi(r)=\max \left\{0, \frac{0.5(r+|r|)\left[(2 \beta-128) r^{5}+(640-4 \beta) r^{4}+2 \beta r^{3}\right]}{(1+|r|)^{9}}\right\} .
$$




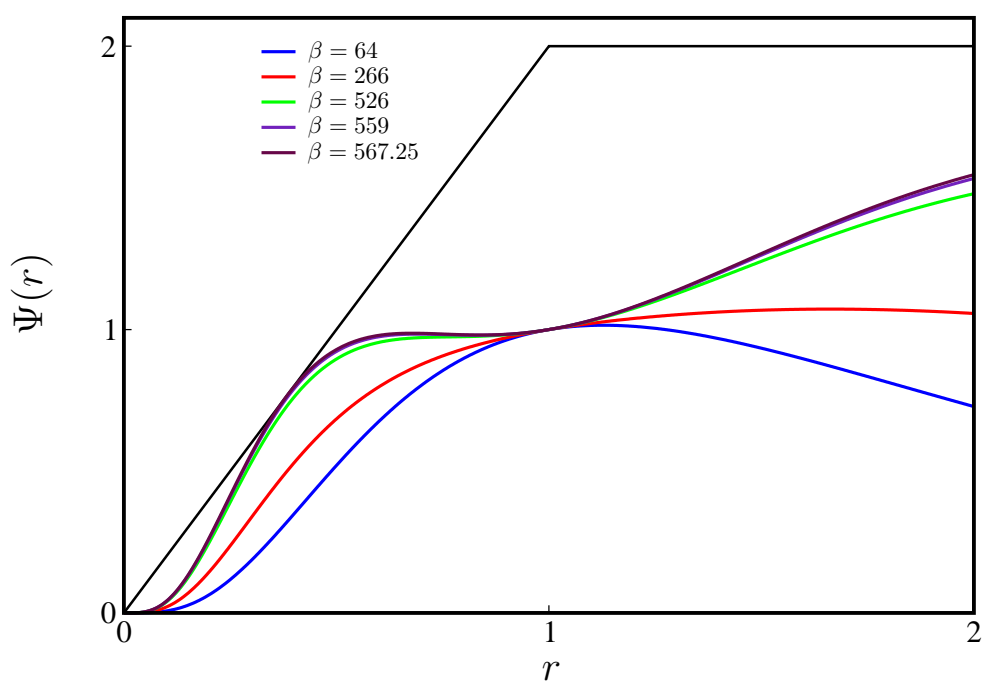

Figura 2.13: Região TVD para o esquema TDPUS-C3 em termos de limitadores de fluxo. 


\section{Capítulo}

\section{Formulação de sistemas hiperbólicos e metodologia de solução}

Nesse capítulo apresentam-se, inicialmente, conceitos básicos de equações de sistemas hiperbólicos de leis de conservação. Algumas definições básicas úteis, tais como solução fraca, problema de Riemann, ondas de choque e de rarefação e descontinuidade de contato, são também apresentadas na sequência. A seguir, são descritos o método de Godunov, o algoritmo de propagação de ondas, uma breve descrição dos resolvedores de Riemann e a discretização das leis de conservação. Por fim, a metodologia de marcha no tempo adotada neste trabalho é discutida.

\subsection{Conceitos básicos}

O problema de valor inicial de trabalho para equações de conservação hiperbólicas é dado por

$$
\begin{gathered}
u_{t}+f(u)_{x}+g(u)_{y}=S(u), \quad 0 \leq t \leq T, \quad a \leq x \leq b, \quad c \leq y \leq d, \\
u(x, y, 0)=u_{0}(x, y),
\end{gathered}
$$

onde $u=\left(u_{1}, u_{2}, \ldots, u_{m}\right)^{T}$ é o vetor das variáveis de estado; $f(u)$ e $g(u)$ são vetores fluxo (com $m$ componentes) nas direções $x$ e $y$, respectivamente; e $S$ é o vetor de termos fonte (ou de origem). Com o objetivo de investigar o desempenho do novo esquema convectivo TDPUS-C3 (que será apresentado no capítulo 5), as seguintes equações hiperbólicas foram consideradas neste trabalho, a saber: advecção de escalares; equação de Burgers 1D e 2D; equação de Buckley-Leverett 1D; equações de Euler 1D e 2D; águas rasas 1D e 2D; e equações da magnetohidrodinâmica 1D e 2D. A partir de agora, a menos que se diga o contrário, o sistema (3.1) será considerado em 1D.

O sistema (3.1) é hiperbólico no sentido de que a matriz jacobiana $A(u)=\partial f(u) / \partial u=f^{\prime}(u)$, é de ordem $m$, tem $m$ auto valores reais da forma $\lambda^{1}(u) \leq \lambda^{2}(u) \leq \cdots \leq \lambda^{m}(u)$ e um conjunto completo 
de $m$ auto vetores linearmente independentes. A forma integral da equação de trabalho (3.1) é

$$
\frac{d}{d t} \int_{b}^{a} u(x, t) d x=f(u(b, t))-f(u(a, t))
$$

cujas características são funções $x^{\prime}(t)$ tais que

$$
\left\{\begin{array}{l}
\frac{d x(t)}{d t}=f^{\prime}(u(x(t), t)), \\
x(0)=x_{0} .
\end{array}\right.
$$

Ao calcular a derivada total de $u$ em relação a $t$ obtém-se

$$
\frac{d u(x(t), t)}{d t}=u_{x} x^{\prime}(t)+u_{t}=u_{x} f^{\prime}(u(x(t), t))+u_{t}=f(u)_{x}+u_{t}=0 .
$$

De (3.2) conclui-se que

$$
\left.u(x(t), t)\right|_{t=0}=u(x(0), 0)=u_{0}\left(x_{0}\right) .
$$

Assume-se que a solução de (3.1)-(3.2) é contínua; mas nem sempre isso acontece. Por exemplo, para a equação de Burgers (onde a função fluxo é $f(u)=0.5 u^{2}$ ) com $u_{0}(x)=\operatorname{sen} x$, as características se interceptam nos pontos $\pi / 2$ e $3 \pi / 2$ e são propagadas com diferentes velocidades. Nesse caso, a solução "global" de (3.4) não está definida. Poder-se-ia obter soluções contínuas, para pequenos valores de $t$ e com condição inicial suave, tais que as características não se interceptam. No entanto, para valores relativamente grandes de $t$, as características podem se cruzar (como acontece na equação de Burgers) e não mais poder-se-ia obter solução clássica de (3.1). Para superar essa falta de existência, deve-se introduzir um conceito mais amplo; isto é o de solução com descontinuidades. Surge assim, neste contexto, o conceito de solução fraca (mais detalhes podem ser encontrados em LeVeque (1992)). Em síntese, uma solução fraca para (3.1) é obtida multiplicando-se a formulação integral (3.3) por uma função suave com suporte compacto $\phi(x, t) \in C_{0}^{1}\left(\mathbb{R} \times \mathbb{R}^{+}\right)$e integrando-se na região $(-\infty, \infty) \times[0, \infty)$; o resultado é uma função limitada $u(x, t)$ que satisfaz (ver Nóvais e de C. Cunha (2003))

$$
\int_{0}^{\infty} \int_{-\infty}^{\infty}\left[u \phi_{t}+f(u) \phi_{x}\right] d x d t+\int_{-\infty}^{\infty} u(x, 0) \phi(x, 0) d x=0
$$

para todas as funções $\phi(x, t) \in C_{0}^{1}(-\infty, \infty) \times[0, \infty)$.

A velocidade (positiva) de propagação de uma descontinuidade, usualmente conhecida como choque (ou onda de choque), é calculada, por exemplo, integrando a lei de conservação (3.1) no retângulo infinitesimal $\left[x_{1}, x_{1}+\delta x\right] \times\left[t_{1}, t_{1}+\delta t\right]$, cujo resultado é

$$
\int_{t_{1}}^{t_{2}} \int_{x_{1}}^{x_{2}}\left(u_{t}+f(u)_{x}\right) d x d t=u_{-} \delta x-u_{+} \delta x+f\left(u_{+}\right) \delta t-f\left(u_{-}\right) \delta t=0,
$$

onde $u_{+}$e $u_{-}$são os valores da velocidade $u(x, t)$ à esquerda e à direita do choque, respectivamente (ver Fig. 3.1). De (3.8) calcula se a velocidade $s$ de choque como

$$
s=\frac{\delta x}{\delta t}=\frac{f\left(u_{+}\right)-f\left(u_{-}\right)}{u_{+}-u_{-}}=\frac{[f]}{[u]} .
$$


A expressão (3.9) é conhecida como a condição de salto de Rankine-Hugoniot (ver, por exemplo, LeVeque (1992)), em que [·] representa o salto de alguma quantidade através da descontinuidade.

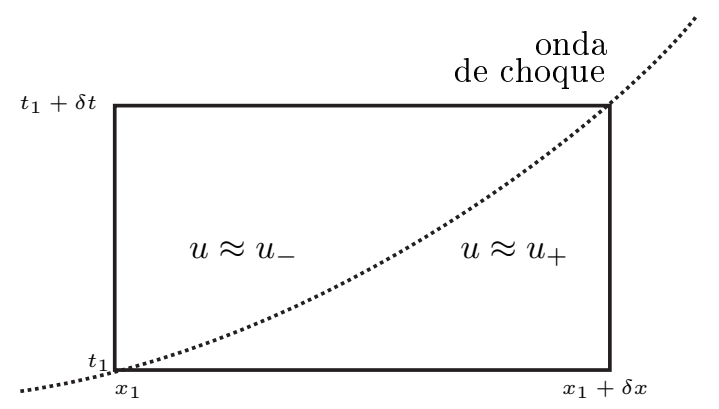

Figura 3.1: Região de integração para o cálculo da velocidade do choque.

O problema de Riemann para a lei de conservação hiperbólica (3.1) é definido como a própria EDP suplementada com a condição inicial

$$
u(x, 0)= \begin{cases}u_{-}, & x \leq x_{0} \\ u_{+}, & x>x_{0}\end{cases}
$$

$\operatorname{com} a<x_{0}<b$.

Em geral, leis de conservação hiperbólicas suportam três tipos de ondas não lineares: choques, rarefações e descontinuidades de contato. A formalização desses conceitos depende da relação entre $u_{-}$e $u_{+}$no problema de Riemann (3.1)-(3.10). No caso em que $f^{\prime}\left(u_{-}\right)>f^{\prime}\left(u_{+}\right)$, a solução (fraca) do problema de Riemann é única e dada por

$$
u(x, t)= \begin{cases}u_{-}, & x<s t \\ u_{+}, & x>s t\end{cases}
$$

A Fig. 3.2 ilustra a onda de choque (à esquerda) e as características (à direita); essas últimas dirigindo-se à solução $x=$ st com o avanço temporal. No caso de $f^{\prime}\left(u_{-}\right)<f^{\prime}\left(u_{+}\right)$, existem infinitas soluções fracas do problema de Riemann viajando com velocidade $s$. Uma solução (estável) é a onda de rarefação definida por

$$
u(x, t)= \begin{cases}u_{-}, & x<f^{\prime}\left(u_{-}\right) \\ w(x / t), & f^{\prime}\left(u_{-}\right) t \leq x \leq f^{\prime}\left(u_{+}\right) t \\ u_{+}, & x>f^{\prime}\left(u_{+}\right) t\end{cases}
$$

onde $w=w(\xi=x / t)$ satisfaz a condição $f^{\prime}(w(\xi))=\xi, \xi=x / t$ (ver Nóvais e de C. Cunha (2003)). A Fig. 3.3 ilustra à direita a onda de rarefação.

Existem também sistemas hiperbólicos que, além de ondas de choque e rarefação, apresentam curvas ou superfícies que separaram regiões com diferentes densidades e/ou temperaturas. Essas estruturas, denominadas descontinuidades de contato (ver, por exemplo, Toro (1999), Déspres e Lagoutiere (2001) e LeVeque (2004)), são caracterizadas por apresentarem pressões de equilíbrio, e portanto não há 
transporte de propriedades através delas. A Fig. 3.4 ilustra, no caso das equações de Euler da dinâmica dos gases, a descontinuidade de contato e também as ondas de rarefação e de choque.
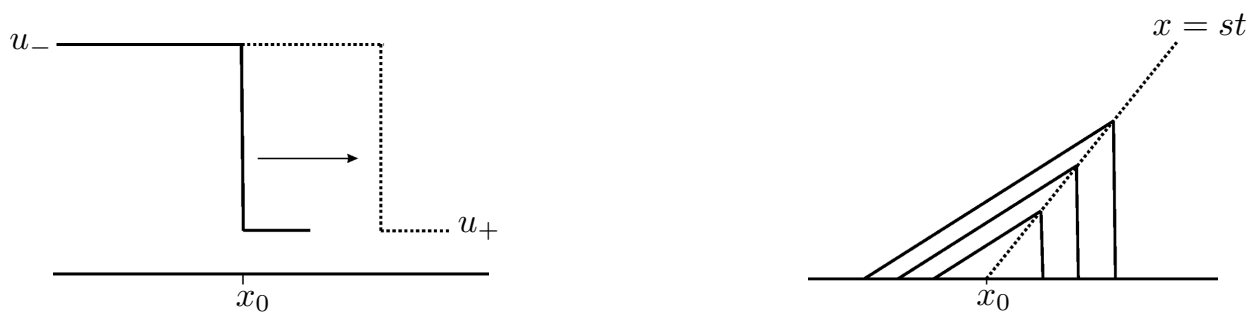

Figura 3.2: Onda de choque.
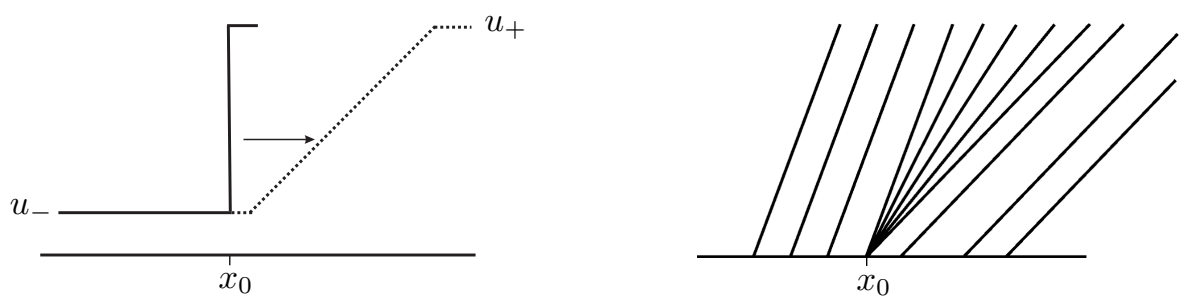

Figura 3.3: Onda de rarefação.

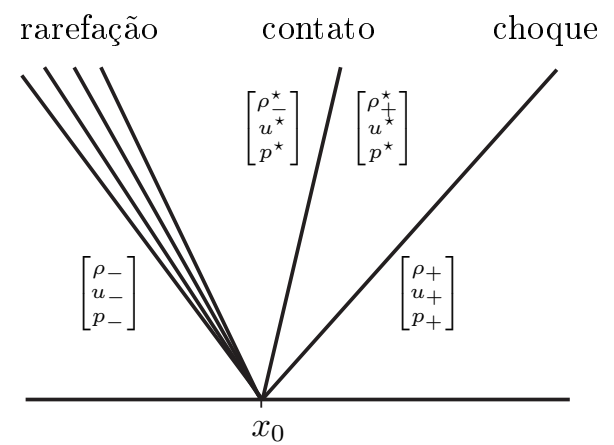

Figura 3.4: Solução típica para um problema de Riemann das equações de Euler.

Quando não há unicidade de solução para a equação de conservação hiperbólica (3.1), pode-se adicionar outras condições para gerar uma solução fraca fisicamente aceitável; essas condições são chamadas condições de entropia. Existem na literatura duas técnicas principais de condições de entropia: a de Lax e a de Oleinik (Shu, 2006). A condição de entropia de Lax impõe que uma descontinuidade propagando-se com velocidade $s$ satisfaz

$$
f^{\prime}\left(u_{-}\right)>s>f^{\prime}\left(u_{+}\right)
$$


A condição de entropia de Oleinik impõe que qualquer função $u(x, t)$ tal que $u_{-} \leq u \leq u_{+}$satisfaz

$$
\frac{f(u)-f\left(u_{-}\right)}{u-u_{-}} \geq s \geq \frac{f(u)-f\left(u_{+}\right)}{u-u_{+}} .
$$

\subsection{Métodos tipo Godunov e algoritmos de propagação de ondas}

Para resolver numericamente sistemas hiperbólicos de leis de conservação considera-se neste trabalho métodos tipo Godunov no contexto de volumes finitos. Por simplicidade apresentam-se estes métodos no caso unidimensional.

Ao integrar a Eq. (3.1) no volume de controle $\left[x_{i-1 / 2}, x_{i+1 / 2}\right] \times\left[t^{n}, t^{n+1}\right]$ mostrado na Fig. 3.5 obtém-se

$$
\begin{array}{r}
\frac{1}{\delta x} \int_{x_{i-1 / 2}}^{x_{i+1 / 2}} u\left(x, t^{n+1}\right) d x=\frac{1}{\delta x} \int_{x_{i-1 / 2}}^{x_{i+1 / 2}} u\left(x, t^{n}\right) d x \\
-\frac{\delta t}{\delta x}\left\{\frac { 1 } { \delta t } \int _ { t ^ { n } } ^ { t ^ { n + 1 } } f \left(u\left(x_{i+1 / 2}, t\right) d t-\frac{1}{\delta t} \int_{t^{n}}^{t^{n+1}} f\left(u\left(x_{i-1 / 2}, t\right) d t\right\},\right.\right.
\end{array}
$$

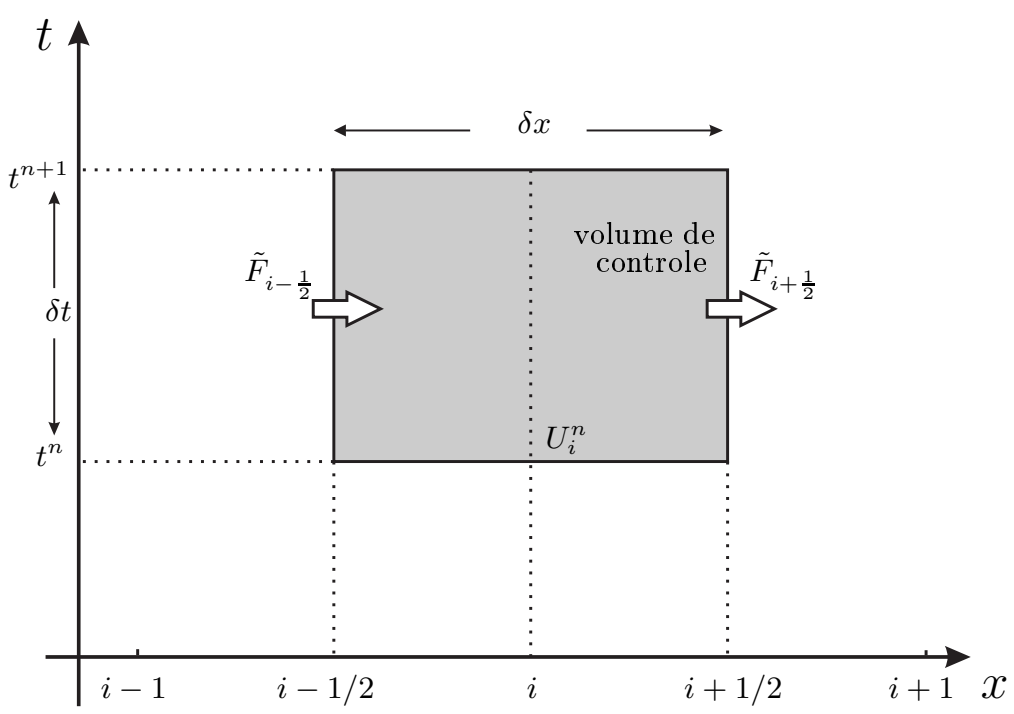

Figura 3.5: Volume de controle no plano $x-t$.

No método de volumes finitos a solução numérica da lei de conservação (3.1) no ponto de malha $(i \delta x, n \delta t)$ é denotada por $U_{i}^{n}$ e aproximada pela média da solução exata $u(x, t)$ no intervalo $\left[x_{i-1 / 2}, x_{i+1 / 2}\right]$, $\operatorname{com} x_{i \pm \frac{1}{2}}=\left(i \pm \frac{1}{2}\right) \delta x$, isto é

$$
U(i \delta x, n \delta t)=U_{i}^{n} \approx \frac{1}{\delta x} \int_{x_{i-1 / 2}}^{x_{i+1 / 2}} u\left(x, t^{n}\right) d x .
$$

As duas últimas quantidades do lado direito da Eq. (3.15) são os fluxos numéricos (ou convectivos) nas interfaces $i+1 / 2$ e $i-1 / 2$ do volume de controle; no caso da primeira quantidade, o fluxo numérico 
é definido por

$$
\tilde{F}\left(U_{i}^{n}, U_{i+1}^{n}\right) \approx \frac{1}{\delta t} \int_{t^{n}}^{t^{n+1}} f\left(u\left(x_{i+\frac{1}{2}}, t\right)\right) d t \equiv \tilde{F}_{i+\frac{1}{2}}
$$

As relações em (3.15), (3.16) e (3.17) podem ser utilizadas para definir um método numérico de aproximação explícito e de três pontos, que na forma conservativa para a lei de conservação (3.1) é dado por

$$
U_{i}^{n+1}=U_{i}^{n}+\frac{\delta t}{\delta x}\left(\tilde{F}_{i-\frac{1}{2}}-\tilde{F}_{i+\frac{1}{2}}\right)
$$

onde $\tilde{F}$ satisfaz a condição de consistência

$$
\tilde{F}\left(U_{i}, U_{i+1}, U_{i-1}, \ldots\right)=F(U) .
$$

O método numérico específico a ser derivado da expressão (3.18) depende de como se escolhe os fluxos numéricos $\tilde{F}_{i \pm \frac{1}{2}}$. Em particular, como será visto na seção 3.3 para as equações de adveç̧ão, Burgers e Buckley-Leverett, a expressão (3.18) pode ser vista como um método numérico em diferenças finitas para a equação (3.1), onde nesse caso a média da solução exata $u(x, t), U_{i}^{n}$, coincide com a própria solução exata $u(x, t)$, isto é $u_{i}^{n}$, e os fluxos numéricos $\tilde{F}$, nas faces $i \pm \frac{1}{2}$, são substituídos pelos próprios valores da função fluxo $f$ nestas faces.

Métodos de tipo Godunov têm como propósito resolver problemas de Riemann nas interfaces, por exemplo em $i+1 / 2$ e $i-1 / 2$, dos volumes de controle e calcular os fluxos numéricos $\tilde{F}_{i-\frac{1}{2}}, \tilde{F}_{i+\frac{1}{2}}$ associados às interfaces. Para tanto, usa-se uma solução aproximada conhecida como resolvedor de Riemann, cujos detalhes podem ser encontrados, por exemplo, em Toro (1999).

Na literatura especializada há uma variedade de resolvedores aproximados de Riemann (ver, por exemplo, Toro (1999)), dentre os quais destacam-se os resolvedores HLL e HLLE. De forma geral, a estrutura dos resolvedores aproximados de Riemann usados neste trabalho consiste de $M_{w}$ ondas $\mathcal{W}^{p}$ propagando-se com velocidades absolutas $s^{p}, p=1,2, \ldots, M_{w}$. Determinado os valores de $u_{-}$e $u_{+}$, os vetores $\mathcal{W}^{p}$ representam o salto em $u$ através de cada onda e a soma desses vetores onda recupera o salto total

$$
[u]=\sum_{p=1}^{M_{w}} \mathcal{W}^{p} .
$$

O método numérico para a lei de conservação conservação (3.18) é obtido impondo-se a condição de conservação (ver LeVeque (2004))

$$
[f]=\sum_{p=1}^{M_{w}} s^{p} \mathcal{W}^{p}
$$

e definindo-se o fluxo numérico por

$$
\tilde{F}_{i+\frac{1}{2}}=f\left(U_{i}\right)+\sum_{p=1}^{M_{w}}\left(s_{i+\frac{1}{2}}^{p}\right)^{-} \mathcal{W}_{i+\frac{1}{2}}^{p}=f\left(U_{i+1}\right)-\sum_{p=1}^{M_{w}}\left(s_{i+\frac{1}{2}}^{p}\right)^{+} \mathcal{W}_{i+\frac{1}{2}}^{p},
$$


onde $s^{+}=\max (s, 0), s^{-}=\min (s, 0)$.

Em resumo, o método numérico tipo upwind (explícito e de primeira ordem) é

$$
U_{i}^{n+1}=U_{i}^{n}-\frac{\delta t}{\delta x}\left(\mathcal{A}^{-} \Delta U_{i+\frac{1}{2}}+\mathcal{A}^{+} \Delta U_{i-\frac{1}{2}}\right)
$$

A notação $\mathcal{A}^{+} \Delta U_{i-\frac{1}{2}}$ refere-se a uma entidade que mede o efeito resultante (ou flutuação) das ondas à direita da interface $i-1 / 2$, ao passo que $\mathcal{A}^{-} \Delta U_{i-\frac{1}{2}}$ mede o efeito resultante das ondas à esquerda da mesma interface. Análise semelhante é feita para as entidades $\mathcal{A}^{ \pm} \Delta U_{i+\frac{1}{2}}$. Em particular, as flutuações em (3.23) são dadas por

$$
\mathcal{A}^{-} \Delta U_{i+\frac{1}{2}}=\sum_{p=1}^{M_{w}}\left(s_{i+\frac{1}{2}}^{p}\right)^{-} \mathcal{W}_{i+\frac{1}{2}}^{p}, \quad \mathcal{A}^{+} \Delta U_{i-\frac{1}{2}}=\sum_{p=1}^{M_{w}}\left(s_{i-\frac{1}{2}}^{p}\right)^{+} \mathcal{W}_{i-\frac{1}{2}}^{p} .
$$

O resolvedor HLL, desenvolvido por Harten et al. (1983), consiste em determinar soluções de Riemann fazendo o uso de duas ondas $\left(M_{w}=2\right)$, com velocidades $a_{-}$e $a_{+}$escolhidas (dentre $s^{1}$ ou $s^{2}$ ) para determinar velocidades máximas e mínimas das características do sistema. As ondas para o método HLL são

$$
\mathcal{W}^{1}=u_{m}-u_{-} ; \quad \mathcal{W}^{2}=u_{+}-u_{m}
$$

onde $u_{m}$ é um estado intermediário da solução $u$, calculado a partir de (3.21), dado por

$$
u_{m}=\frac{a_{+} u_{+}-a_{-} u_{-}-\left[f\left(u_{+}\right)-f\left(u_{-}\right)\right]}{a_{+}-a_{-}} .
$$

O resolvedor HLLE foi introduzido por Einfeldt (1988) no contexto da dinâmica dos gases e é uma modificação do resolvedor HLL, em que as velocidades $a_{-}$e $a_{+}$são definidas por

$$
a_{-}=\min _{p}\left(\min \left(\lambda^{p}\left(u_{-}\right), \hat{\lambda}^{p}\left(u_{-}, u_{+}\right)\right)\right), \quad a_{+}=\max _{p}\left(\max \left(\lambda^{p}\left(u_{+}\right), \hat{\lambda}^{p}\left(u_{-}, u_{+}\right)\right)\right),
$$

onde $\lambda^{p}(u)$ é o $p$-ésimo autovalor da matriz jacobiana $A(u)$ do sistema, e $\hat{\lambda}^{p}\left(u_{-}, u_{+}\right)$é o $p$-ésimo autovalor da linearização no método de Roe (ver Roe (1981)).

Os algoritmos de propagação de ondas de LeVeque (1997) propõem modificar o método de primeira ordem de Godunov (3.23) introduzindo um termo de correção para atingir ordem maior. Estes algoritmos para o caso 1D (por simplicidade) são da forma

$$
U_{i}^{n+1}=U_{i}^{n}-\frac{\delta t}{\delta x}\left(\mathcal{A}^{-} \Delta U_{i+\frac{1}{2}}+\mathcal{A}^{+} \Delta U_{i-\frac{1}{2}}\right)-\frac{\delta t}{\delta x}\left(\widetilde{F}_{i+\frac{1}{2}}-\widetilde{F}_{i-\frac{1}{2}}\right)
$$

onde $\widetilde{F}_{i+\frac{1}{2}}$ e $\widetilde{F}_{i-\frac{1}{2}}$ são as correções de segunda ordem. Por exemplo, a correção $\widetilde{F}_{i+\frac{1}{2}}$ é dada por

$$
\widetilde{F}_{i+\frac{1}{2}}=\frac{1}{2}\left|\sum_{p=1}^{M_{w}}\left(s_{i+\frac{1}{2}}^{p}\right)\right|\left(1-\frac{\delta t}{\delta x}\left|s_{i+\frac{1}{2}}^{p}\right|\right) \widetilde{\mathcal{W}}_{i+\frac{1}{2}}^{p}
$$

onde $\widetilde{\mathcal{W}}_{i+\frac{1}{2}}^{p}$ é definido ao se multiplicar a onda $\mathcal{W}_{i+\frac{1}{2}}^{p}$ por um limitador de fluxo $\Psi$, tal como o limitador 
TDPUS-C3 proposto no capítulo 2, isto é

$$
\widetilde{\mathcal{W}}_{i+\frac{1}{2}}^{p}=\Psi\left(r_{i+\frac{1}{2}}\right) \mathcal{W}_{i+\frac{1}{2}}^{p}
$$

O indicador de suavidade $r_{i+\frac{1}{2}}$ é obtido ao se comparar as ondas $\mathcal{W}_{i+\frac{1}{2}}^{p}$ e $\widetilde{\mathcal{W}}_{I+\frac{1}{2}}^{p}$ da mesma família $p$ na direção upwind I (os detalhes podem ser encontrados em LeVeque (1997))

$$
I= \begin{cases}i-1, & s_{i+\frac{1}{2}}^{p}>0 \\ i+1, & s_{i+\frac{1}{2}}^{p}<0 .\end{cases}
$$

Neste trabalho, a seguinte expressão para a variável $r_{i+\frac{1}{2}}$ é utilizada

$$
r_{i+\frac{1}{2}}=\frac{\widetilde{\mathcal{W}}_{I+\frac{1}{2}}^{p} \cdot \widetilde{\mathcal{W}}_{i+\frac{1}{2}}^{p}}{\widetilde{\mathcal{W}}_{i+\frac{1}{2}}^{p} \cdot \widetilde{\mathcal{W}}_{i+\frac{1}{2}}^{p}}
$$

Outras abordagens para a variável $r_{i+\frac{1}{2}}$ podem ser encontradas em Liu e Lax (1996) e Lax e Liu (1998).

O código CLAWPACK usado para resolver numericamente as leis de conservação hiperbólica e sistemas não conservativos hiperbólicos é baseado nos algoritmos de propagação das ondas. Quando os valores dos fluxos $\widetilde{F}_{i+\frac{1}{2}}$ e $\widetilde{F}_{i+\frac{1}{2}}$ são iguais a zero em (3.28), obtêm-se o esquema de primeira ordem de Godunov.

No caso multidimensional, a metodologia a usar é similar ao caso $1 \mathrm{D}$, ver para mais detalhes LeVeque (1997).

\subsection{Discretização das leis de conservação}

Nesta seção, usando os métodos de diferenças e volumes finitos, são apresentadas as discretizações dos sistemas hiperbólicos de leis de conservação estudados neste trabalho. No caso das equações de advecção, Burgers e Buckley-Leverett, as discretizações foram feitas no contexto de diferenças finitas usando esquemas upwind na normalização de Leonard. As discretizações feitas nas equações de águas rasas, Euler e magnetohidrodinâmica foram no contexto de volumes finitos usando os correspondentes limitadores de fluxo dos esquemas upwind.

\subsubsection{Advecção de escalares}

Em advecção escalares, a função fluxo na Eq. (3.1) é $f(u)=a u$ considerando $a>0$ constante. O fluxo numérico na face $i+\frac{1}{2}$ (na face $i-\frac{1}{2}$ o procedimento de cálculo é semelhante) é aproximado por

$$
f(u)_{i+\frac{1}{2}}=\left.(a u)\right|_{i+\frac{1}{2}}=a_{i+\frac{1}{2}} \cdot u_{i+\frac{1}{2}}=a \cdot u_{i+\frac{1}{2}},
$$

onde $u_{i+\frac{1}{2}}$ é interpolado (usando o sinal da velocidade de advecção $a$ ) em função dos três pontos $D=i+1, U=i$ e $R=i-1$ da malha computacional. Por exemplo, usando o esquema TDPUS-C3 
uma aproximação para $u_{i+\frac{1}{2}}$ é obtida como

$$
u_{i+\frac{1}{2}}=\left\{\begin{array}{cc}
u_{R}+\left(u_{D}-u_{R}\right) *\left[\hat{u}_{U}+\hat{u}_{U}^{4}\left(\alpha_{4}+\hat{u}_{U}\left(\alpha_{5}+\hat{u}_{U}\left(\alpha_{6}+\hat{u}_{U}\left(\alpha_{7}+\right.\right.\right.\right.\right. & \\
\left.\left.\left.\left.\hat{u}_{U}\left(\alpha_{8}+\hat{u}_{U}\left(\alpha_{9}+\hat{u}_{U} \alpha_{10}\right)\right)\right)\right)\right)\right], & \hat{u}_{U} \in[0,1] ; \\
u_{U}, & \hat{u}_{U} \notin[0,1],
\end{array}\right.
$$

em que $\hat{u}_{U}$ é a variável normalizada de Leonard no ponto (à montante) $U$ e definida usando (2.6) e as constantes $\alpha_{i=4, \ldots, 10}$ definidas em (2.56).

\subsubsection{Equação de Burgers}

No caso da equação de Burgers 1D (o caso 2D segue procedimento análogo), a função fluxo é dada por $f(u)=0.5 u^{2}$ e a velocidade de conveç̧ão $\bar{u}_{i+\frac{1}{2}}$ é definida pela fórmula de Roe (Ahmed, 2004)

$$
\bar{u}_{i+\frac{1}{2}}= \begin{cases}\frac{\delta t}{\delta x} \frac{f_{i+1}-f_{i}}{u_{i+1}-u_{i}}, & u_{i+1} \neq u_{i} ; \\ \frac{\delta t}{\delta x} f^{\prime}\left(u_{i}\right), & u_{i+1}=u_{i} .\end{cases}
$$

Por exemplo, o fluxo numérico $f_{i+\frac{1}{2}}$ (o caso $f_{i-\frac{1}{2}}$ segue procedimento semelhante) usando o esquema TDPUS-C3 é aproximado como

$$
f_{i+\frac{1}{2}}= \begin{cases}f\left(u_{R}\right)+\left(f\left(u_{D}\right)-f\left(u_{R}\right)\right) *\left[\hat{f}\left(u_{U}\right)+\hat{f}\left(u_{U}\right)^{4}\left(\alpha_{4}+\hat{f}\left(u_{U}\right)\left(\alpha_{5}+\right.\right.\right. & \\ \left.\left.\hat{f}\left(u_{U}\right)\left(\alpha_{6}+\hat{f}\left(u_{U}\right)\left(\alpha_{7}+\hat{f}\left(u_{U}\right)\left(\alpha_{8}+\hat{f}\left(u_{U}\right)\left(\alpha_{9}+\hat{f}\left(u_{U}\right) \alpha_{10}\right)\right)\right)\right)\right)\right], & \hat{f}\left(u_{U}\right) \in[0,1] ; \\ f\left(u_{U}\right), & \hat{f}\left(u_{U}\right) \notin[0,1],\end{cases}
$$

onde $\hat{f}\left(u_{U}\right)$ (a função fluxo normalizada) é dada por

$$
\hat{f}\left(u_{U}\right)=\frac{f\left(u_{U}\right)-f\left(u_{R}\right)}{f\left(u_{D}\right)-f\left(u_{R}\right)}
$$

A discretização da equação de Buckley-Leverett é similar à discretização feita para a equação de Burgers, cuja diferença está no cálculo da função fluxo $f(u)$ (não convexa).

\subsubsection{Equações das águas rasas}

Nos algoritmos de propagação de ondas dados por (3.28)-(3.32), o fluxo numérico $\tilde{F}_{i+\frac{1}{2}}$ é obtido via a Eq. (3.29) com o auxílio (por exemplo) do correspondente limitador de fluxo $\Psi$ para o esquema TDPUS-C3. Nos outros sistemas hiperbólicos (Euler e magnetohidrodinâmica), os fluxos numéricos são aproximados de maneira similar.

\subsection{A marcha no tempo}

Para a simulação computacional de leis de conservação 1D e 2D transientes, nesse trabalho, foram utilizados dois métodos explícitos para a marcha no tempo: (i) o método de Euler; e (ii) o método de Runge-Kutta de terceira ordem strong stability preserving Runge-Kutta 3 (SSP-RK3) (ver, por exemplo, Gottlieb e Chi-Wang-Shu (1998) e Gottlieb et al. (2001)). O método de Euler está implementado 
no código CLAWPACK (ver Eq. 3.28). O método SSP-RK3 está implementado no código próprio (ver apêndice B) e é dado por

$$
\begin{aligned}
u^{(1)} & =u^{n}+\Delta t L\left(u^{n}\right), \\
u^{(2)} & =\frac{3}{4} u^{n}+\frac{1}{4} u^{(1)}+\frac{1}{4} \Delta t L\left(u^{(1)}\right), \\
u^{(n+1)} & =\frac{1}{3} u^{n}+\frac{2}{3} u^{(2)}+\frac{2}{3} \Delta t L\left(u^{(2)}\right),
\end{aligned}
$$

onde $L(u)$ ó o operador espacial da Eq. (3.1).

Nem sempre o método de Euler é útil para resolver sistemas hiperbólicos; de fato pois, de um lado ao resolver a Eq. (3.1) com $f(u)=0.5 u^{2}$ (equação de Burgers $1 \mathrm{D}$ sem viscosidade) e condição inicial $\operatorname{com} u_{0}(x, 0)=1+\frac{1}{2} \sin (\pi x)$ usando a marcha no tempo por Euler (a $C F L=0.5$ e tempo final $t=0.33$ ) para diferentes resoluções espaciais $(N=100,200,300,400$ e 500), obtém-se o resultado mostrado na Fig. 3.6. Vê-se claramente por esta figura que o método de Euler forneceu resultados oscilatórios,

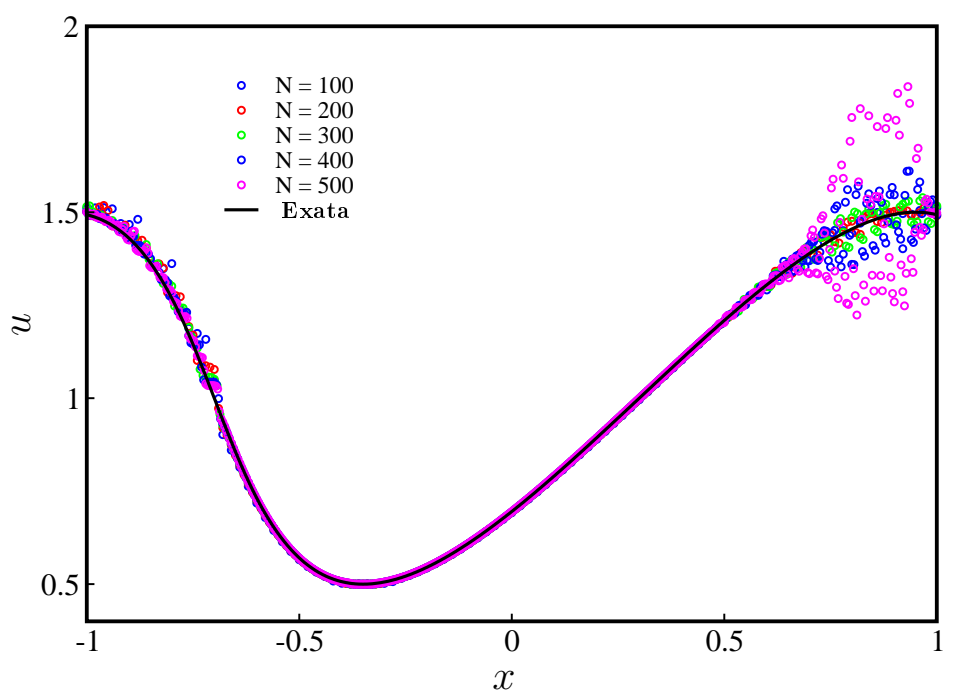

Figura 3.6: Evolução espacial das soluções numéricas da equação de Burgers 1D calculadas com o esquema TDPUS-C3 e $\beta=559$. Euler explícito na parte temporal (não TVD). $C F L=0.5, t=0.33$.

piorando cada vez mais em malhas refinadas. Por outro lado, ao se aplicar o método SSP-RK3 neste mesmo problema com uma resolução espacial de $N=500$ células computacionais obtém-se o resultado apresentado na Fig. 3.7, onde pode-se ver que a solução exata foi praticamente reproduzida com o uso do método SSP-RK3.

Portanto, fica claro, por este teste, que um método de Runge-Kutta de alta ordem é fortemente recomendado para a marcha no tempo em leis de conservação hiperbólicas.

Em virtude da marcha no tempo ser explícita, os métodos numéricos para as equações de advecção, Burgers e Buckley-Leverett têm a seguinte restrição de estabilidade: $C F L \leq 1$. Para as equações de águas rasas, Euler e magnetohidrodinâmica, a restrição de estabilidade utilizada é (ver, por exemplo, 


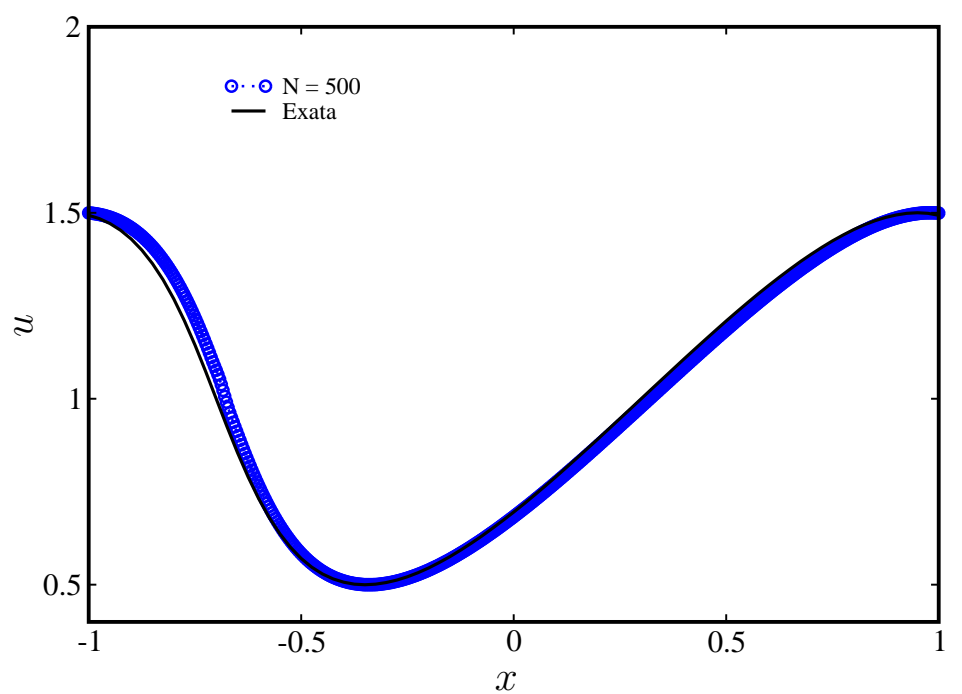

Figura 3.7: Soluções numéricas da equação de Burgers 1D calculadas com o esquema TDPUS-C3 e $\operatorname{com} \beta=559$. SSP-RK3 na parte temporal (TVD). $t=0.33, N=500$ e $C F L=0.5$.

Pelanti (2005))

$$
C F L=\frac{\delta t}{\delta x} \max _{1 \leq p \leq M_{w}}\left|s^{p}\right| \leq 1
$$

\subsection{Avaliação dos erros}

Para avaliação dos erros e análise das soluções numéricas calculadas ao implementar os diferentes esquemas convectivos nas leis de conservação 1D e 2D, foram utilizadas as definições que seguem. Seja $u\left(x, t^{n}\right)$ e $U\left(x, t^{n}\right)$ as soluções numérica e exata, respectivamente, em um ponto $(x, t)$. O erro absoluto $E$ é dado por

$$
E=|U-u|
$$

Com o propósito de quantificar os erros, definem-se duas normas

$$
\begin{aligned}
& L_{1} \quad \text { erro: } \quad\|E\|_{1}=\quad \sum_{i=1}^{N}\left|U\left(x, t^{n}\right)-u\left(x, t^{n}\right)\right| \delta x ; \\
& L_{\infty} \text { erro: } \quad\|E\|_{\infty}=\quad \max _{1 \leq j \leq N}\left|U\left(x, t^{n}\right)-u\left(x, t^{n}\right)\right| \text {. }
\end{aligned}
$$

O erro relativo $E$ é dado por

$$
E=\frac{|U-u|}{|U|}
$$

onde $U$, na ausência da solução exata, pode ser uma solução calculada em uma malha fina.

Como comentário geral sobre as normas escolhidas, a norma $L_{1}$ dá uma visão mais global dos erros, enquanto que a norma $L_{\infty}$ enfatiza os erros que aparecem nos picos ou nas descontinuidades (Greenough e Rider, 2004). 
Neste trabalho assumimos que o erro, em função de $\delta x$, se comporta assintoticamente como

$$
E(h) \approx C \delta x^{q}, \quad \text { quando } \quad \delta x \rightarrow 0,
$$

onde $C$ é a constante assintótica do erro, $q$ corresponde a ordem de convergência observada do método numérico e $h$ é o espaçamento da malha.

A ordem de convergência $q$ é estimada pela fórmula

$$
q \approx \frac{\log \left(\frac{E(h)}{E(h / 2)}\right)}{\log 2} .
$$

No caso em que não se conhece a solução exata a estimativa para ordem de convergência pode ser calculada com base em três soluções numéricas obtidas em malhas com espaçamentos $\delta x, \delta x / 2$ e $\delta x / 4$

$$
q \approx \frac{\log \left(\frac{\left\|u^{\delta x / 2}-u^{\delta x}\right\|}{\left\|u^{\delta x / 4}-u^{\delta x / 2}\right\|}\right)}{\log 2},
$$

em que $u^{\delta x}, u^{\delta x / 2}$ e $u^{\delta x / 4}$ são, respectivamente, a solução numérica na malha grossa, a solução numérica na malha intermediária e a solução numérica na malha fina. 


\section{Capítulo}

\section{Formulação de escoamentos incompressíveis, metodologia de solução e equações discretas}

Nesse capítulo é apresentada a formulação matemática (nos sistemas de coordenadas gerais e cartesianas) de escoamentos incompressíveis newtonianos (nos regimes laminar e turbulento) e não newtonianos laminares. A seguir, são descritos a metodologia MAC e o algoritmo de solução para escoamentos incompressíveis. Por fim, a discretização das equações governantes e a marcha no tempo são apresentadas.

\subsection{Equações básicas}

As equações básicas, na forma conservativa dimensional, que modelam escoamentos incompressíveis ( $\rho$ - constante) são definidas por (White, 2006)

$$
\begin{aligned}
\frac{\partial \mathbf{u}}{\partial t}+\nabla \cdot(\mathbf{u u}) & =\frac{1}{\rho} \nabla \cdot \boldsymbol{\sigma}+\mathbf{g}, \\
\nabla \cdot \mathbf{u} & =0,
\end{aligned}
$$

onde $\mathbf{u}(\mathbf{x}, t)=\left(u_{1}(\mathbf{x}, t), u_{2}(\mathbf{x}, t), u_{3}(\mathbf{x}, t)\right)^{T}$ é o vetor velocidade (em coordenadas cartesianas esse vetor é denotado por $\left.(u, v, w)^{T}\right)$ de uma partícula de fluido na posição $\mathbf{x}$ e no tempo $t ; p=p(\mathbf{x}, t)$ a pressão e $\mathbf{g}$ a aceleração gravitacional com intensidade $g$. O tensor de tensões $\boldsymbol{\sigma}$ no caso de fluidos newtonianos é definido por

$$
\sigma=-p \mathbb{I}+\boldsymbol{\tau}
$$


com II sendo o tensor identidade e $\boldsymbol{\tau}$ o tensor de cisalhamento dado por

$$
\boldsymbol{\tau}=\mu \underbrace{\left[\nabla \mathbf{u}+(\nabla \mathbf{u})^{T}\right]}_{\mathbf{D}}=\mu \mathbf{D},
$$

onde $\mu$ é o coeficiente de viscosidade molecular do fluido (constante) e $\mathbf{D}$ é o tensor de deformações. No caso de fluidos não newtoniano, o tensor de tensões $\boldsymbol{\sigma}$ é expresso como

$$
\boldsymbol{\sigma}=-p \mathbb{I}+2 \mu_{s} \mathbf{D}+\boldsymbol{\tau}_{p}
$$

com $\mu_{s}$ a viscosidade do solvente e $\boldsymbol{\tau}_{p}$ o tensor do polímero que é determinado por uma equação constitutiva a ser apresentada no seção 4.1.3. As equações (4.1) e (4.2) são as denominadas equações de momento e continuidade. As mudanças de variáveis para adimensionalização das Eqs. (4.1)-(4.5) são definidas por:

$$
\begin{aligned}
& \mathbf{x}^{\star}=\frac{\mathbf{x}}{L_{0}}, \quad t^{\star}=\frac{t_{0} U_{0}}{L_{0}}, \quad \mathbf{u}^{\star}=\frac{\mathbf{u}}{U_{0}}, \quad p^{\star}=\frac{p}{\rho_{0} U_{0}^{2}}, \\
& \mathbf{g}^{\star}=\frac{\mathbf{g}}{g}, \quad \boldsymbol{\tau}_{p}^{\star}=\frac{\boldsymbol{\tau}_{p}}{\rho U_{0}^{2}},
\end{aligned}
$$

onde $L_{0}$ e $U_{0}$ são as escalas de comprimento e velocidade, respectivamente, e $t_{0}$ escala de tempo. Os adimensionais (mais relevantes) que surgem no processo de adimensionalização são:

- Número de Reynolds, Re,

$$
R e=\frac{\rho U_{0} L_{0}}{\mu}=\frac{U_{0} L_{0}}{(\mu / \rho)}=\frac{U_{0} L_{0}}{\nu},
$$

$\nu$ sendo a viscosidade cinemática. Para fluidos não newtonianos $\mu=\mu_{s}+\mu_{p}$, em que $\mu_{p}$ é a viscosidade do polímero;

- Número de Froude, Fr,

$$
F r=\frac{U_{0}}{\sqrt{g L_{0}}}
$$

- Número de Weissemberg, We,

$$
W e=\frac{\lambda_{1} U_{0}}{L_{0}},
$$

sendo $\lambda_{1}$ uma constante temporal para fluidos viscoelásticos;

- Razão entre as viscosidades, $\vartheta$, (para fluidos não newtonianos)

$$
\vartheta=\frac{\mu_{s}}{\mu}, \quad \vartheta \in(0,1)
$$

- Razão entre constantes temporais de relaxação, (para fluidos não newtonianos) $\gamma$,

$$
\gamma=\frac{\lambda_{2}}{\lambda_{1}}
$$




\subsubsection{Formulação matemática para fluidos newtonianos}

As equações básicas de trabalho, em coordenadas cartesianas, para escoamentos incompressíveis de fluidos newtonianos são obtidas da equação do movimento (4.1) e conservação de massa (4.2), com o auxílio das equações (4.3) e (4.4) para o tensor de tensão e de cisalhamento, respectivamente. Na forma dimensional e em notação de Einstein (indicial) essas equações (de Navier-Stokes) no caso 3D são expressas como

$$
\begin{gathered}
\frac{\partial u_{i}}{\partial t}+\frac{\partial\left(u_{i} u_{j}\right)}{\partial x_{j}}=-\frac{1}{\rho} \frac{\partial p}{\partial x_{i}}+\nu \frac{\partial}{\partial x_{j}}\left(\frac{\partial u_{i}}{\partial x_{j}}\right)+g_{i}, \quad i=1,2,3, \\
\frac{\partial u_{i}}{\partial x_{i}}=0 .
\end{gathered}
$$

Na forma expandida adimensional, as equações de trabalho (4.12) e (4.13) tornam-se

$$
\begin{gathered}
\frac{\partial u}{\partial t}+\frac{\partial(u u)}{\partial x}+\frac{\partial(u v)}{\partial y}+\frac{\partial(u w)}{\partial z}=-\frac{\partial p}{\partial x}+\frac{1}{R e}\left(\frac{\partial^{2} u}{\partial x^{2}}+\frac{\partial^{2} u}{\partial y^{2}}+\frac{\partial^{2} u}{\partial z^{2}}\right)+\frac{1}{F r^{2}} g_{x} \\
\frac{\partial v}{\partial t}+\frac{\partial(v u)}{\partial x}+\frac{\partial(v v)}{\partial y}+\frac{\partial(v w)}{\partial z}=-\frac{\partial p}{\partial y}+\frac{1}{R e}\left(\frac{\partial^{2} v}{\partial x^{2}}+\frac{\partial^{2} v}{\partial y^{2}}+\frac{\partial^{2} v}{\partial z^{2}}\right)+\frac{1}{F r^{2}} g_{y} \\
\frac{\partial w}{\partial t}+\frac{\partial(w u)}{\partial x}+\frac{\partial(w v)}{\partial y}+\frac{\partial(w w)}{\partial z}=-\frac{\partial p}{\partial z}+\frac{1}{R e}\left(\frac{\partial^{2} w}{\partial x^{2}}+\frac{\partial^{2} w}{\partial y^{2}}+\frac{\partial^{2} w}{\partial z^{2}}\right)+\frac{1}{F r^{2}} g_{z} \\
\frac{\partial u}{\partial x}+\frac{\partial v}{\partial y}+\frac{\partial w}{\partial z}=0
\end{gathered}
$$

em que $g_{x}, g_{y}$ e $g_{z}$ são os componentes do campo gravitacional $\mathbf{g}$ nas direções $x, y$ e $z$, respectivamente.

$\mathrm{Na}$ forma adimensional, e em sistema de coordenadas gerais, as equações de Navier-Stokes são dadas por

$$
\begin{aligned}
\frac{\partial \mathbf{u}}{\partial t}+\nabla \cdot(\mathbf{u u}) & =-\nabla p+\frac{1}{R e} \nabla^{2} \mathbf{u}+\frac{1}{F r^{2}} \mathbf{g}, \\
\nabla \cdot \mathbf{u} & =0 .
\end{aligned}
$$

\subsubsection{Modelagem matemática para fluidos newtonianos em regime turbulento}

A modelagem matemática usada neste trabalho para a simulação computacional de escoamentos incompressíveis de fluidos newtonianos em regime turbulento (as denominadas equações Navier-Stokes com média de Reynolds-URANS) são obtidas fazendo-se o uso da decomposição (em componentes média e estocástica) de escalas de Reynolds dada por

$$
\Phi(\mathbf{x}, t)=\bar{\Phi}(\mathbf{x}, t)+\Phi^{\prime}(\mathbf{x}, t)
$$

onde $\Phi^{\prime}(\mathbf{x}, t)$ é a componente flutuante da decomposição e $\bar{\Phi}(\mathbf{x}, t)$ é a componente média definida por

$$
\bar{\Phi}(\mathbf{x}, t)=\lim _{T \rightarrow \infty} \frac{1}{T} \int_{t}^{t+T} \Phi(\mathbf{x}, \tau) d \tau, \quad T_{0} \ll T \ll T_{1},
$$


com $T$ suficientemente grande em relação a escala do tempo $T_{0}$ das flutuações turbulentas, porém pequeno em relação ao tempo $T_{1}$ dos outros efeitos com relação ao valor médio. A ideia geral em se usar essa aproximação é estudar o comportamento do escoamento turbulento em termos de soluções bem comportadas $\bar{\Phi}(\mathbf{x}, t)$. Em resumo, aplicando a decomposição de escalas de Reynolds (4.20) às variáveis instantâneas $u, v, w$ e $p$ das Eqs. dimensionais (4.12) e (4.13) obtém-se as equações de Navier-Stokes com média de Reynolds (ou simplesmente equações médias de Reynolds), as quais em coordenadas cartesianas são dadas por

$$
\begin{gathered}
\frac{\partial \bar{u}_{i}}{\partial t}+\frac{\partial\left(\bar{u}_{i} \bar{u}_{j}\right)}{\partial x_{j}}=-\frac{1}{\rho} \frac{\partial \bar{p}}{\partial x_{i}}+\frac{\partial}{\partial x_{j}}\left(\nu \frac{\partial \bar{u}_{i}}{\partial x_{j}}-\overline{u_{i}^{\prime} u_{j}^{\prime}}\right)+g_{i}, \quad i=1,2,3 \\
\frac{\partial \bar{u}_{i}}{\partial x_{i}}=0
\end{gathered}
$$

onde $\overline{u_{i}^{\prime} u_{j}^{\prime}}$ é o tensor (simétrico) de tensões turbulentas de Reynolds modelado neste trabalho usando a aproximação de Boussinesq

$$
-\overline{u_{i}^{\prime} u_{j}^{\prime}}=2 \nu_{t} \bar{D}_{i j}-\frac{2}{3} \bar{\kappa} \delta_{i j}=\nu_{t}(\underbrace{\frac{\partial \bar{u}_{i}}{\partial x_{j}}+\frac{\partial \bar{u}_{j}}{\partial x_{i}}}_{\bar{D}_{i j}})-\frac{2}{3} \bar{\kappa} \delta_{i j},
$$

$\operatorname{com} \bar{\kappa}=\frac{1}{2} \overline{u_{i}^{\prime} u_{j}^{\prime}}$ a energia cinética média e $\delta_{i j}$ o delta de Kronecker. $\bar{D}_{i j}$ é o tensor médio de deformações. A viscosidade turbulenta $\nu_{t}$ é modelada dimensionalmente por (veja Tennekes e Lumley (1972))

$$
\nu_{t} \sim l_{t} u_{t}=C_{\mu} \kappa T_{t}
$$

onde $C_{\mu}$ é uma constante empírica e as escalas características de comprimento, de velocidade e de tempo são dadas respectivamente por

$$
l_{t} \sim \frac{\bar{\kappa}^{3 / 2}}{\bar{\varepsilon}}, \quad u_{t} \sim \bar{\kappa}^{1 / 2} \quad \text { e } \quad T_{t} \sim \frac{l_{t}}{u_{t}}=\frac{\bar{\kappa}}{\bar{\varepsilon}} .
$$

Na Eq. (4.26), a grandeza $\bar{\varepsilon}$ é a taxa média de dissipação de energia cinética média e definida por

$$
\bar{\varepsilon}=\nu \overline{\frac{\partial u_{i}^{\prime}}{\partial x_{j}} \frac{\partial u_{i}^{\prime}}{\partial x_{j}}}
$$

Substituindo a Eq. (4.24) na Eq. (4.22), obtém-se um sistema não fechado de quatro equações a seis incógnitas $\bar{u}, \bar{v}, \bar{w}, \bar{p}, \bar{\kappa}$ e $\bar{\varepsilon}$. Para o fechamento, deve-se introduzir duas EDPs de transporte; uma para $\bar{k}$ e outra para $\bar{\varepsilon}$. Em resumo, as equações de trabalho na forma adimensional para a simulação computacional de escoamentos incompressíveis de fluidos newtonianos em regime turbulento são, em coordenadas cartesianas, dadas por

$$
\begin{gathered}
\frac{\partial \bar{u}_{i}}{\partial t}+\frac{\left(\bar{u}_{i} \bar{u}_{j}\right)}{\partial x_{j}}=-\frac{\partial p_{e}}{\partial x_{i}}+\frac{1}{R e} \frac{\partial}{\partial x_{j}}\left(\frac{\partial \bar{u}_{i}}{\partial x_{j}}\right)+\frac{1}{F r^{2}} g_{i}+\frac{1}{R e} \frac{\partial}{\partial x_{j}}\left(\nu_{t} \bar{D}_{i j}\right), i=1,2,3 ; \\
\frac{\partial \bar{u}_{i}}{\partial x_{i}}=0
\end{gathered}
$$




$$
\begin{gathered}
\frac{\partial \bar{\kappa}}{\partial t}+\frac{\partial\left(\overline{\kappa u}_{j}\right)}{\partial x_{j}}=\frac{1}{R e} \frac{\partial}{\partial x_{j}}\left(\left(1+\frac{\nu_{t}}{\sigma_{\kappa}}\right) \frac{\partial \bar{\kappa}}{\partial x_{j}}\right)+P-\bar{\varepsilon} \\
\frac{\partial \bar{\varepsilon}}{\partial t}+\frac{\partial\left(\overline{\varepsilon u}_{j}\right)}{\partial x_{j}}=\frac{1}{R e} \frac{\partial}{\partial x_{j}}\left(\left(1+\frac{\nu_{t}}{\sigma_{\varepsilon}}\right) \frac{\partial \bar{\varepsilon}}{\partial x_{j}}\right)+\left(C_{1 \varepsilon} P-C_{2 \varepsilon} \bar{\varepsilon}\right) / T_{t}-\zeta \mathcal{R}, \\
\nu_{t}=C_{\mu} \frac{\bar{\kappa}^{2}}{\bar{\varepsilon}} .
\end{gathered}
$$

Na Eq. (4.28) $p_{e}$ a pressão efetiva média definida como a soma da pressão média e a pressão turbulenta $\bar{p}_{t}$, isto é

$$
p_{e}=\bar{p}+\bar{p}_{t}
$$

O escalar P que aparece nas Eqs. (4.30) e (4.31) é a produção turbulenta e calculada como

$$
P=\frac{\nu_{t}}{2} \bar{D}_{i j} \bar{D}_{i j}
$$

O termo adicional $\mathcal{R}$ na Eq. (4.31) é definido por

$$
\mathcal{R}=\frac{C_{\mu} \eta^{3}\left(1-\eta / \eta_{0}\right)}{1+\varsigma \eta^{3}} \frac{\bar{\varepsilon}^{2}}{\bar{k}}
$$

onde $\eta=S \frac{\bar{\kappa}}{\bar{\varepsilon}}$; sendo $S$ a magnitude da taxa média de tensão e definida como $S=\sqrt{\frac{1}{2} \bar{D}_{i j} \bar{D}_{i j}}$. As variáveis $\nu_{t}, \bar{\kappa}$ e $\bar{\varepsilon}$ nas Eqs. (4.28), (4.30), (4.31) e (4.32) estão na forma adimensional as quais foram obtidas das transformações

$$
\nu_{t}^{\star}=\frac{\nu_{t}}{\nu}, \quad \bar{\kappa}^{\star}=\frac{L_{0} \bar{\kappa}}{\nu U_{0}}, \quad \bar{\varepsilon}^{\star}=\frac{L_{0}^{2} \bar{\varepsilon}}{\nu U_{0}^{2}} .
$$

Na Eq. (4.31), o parâmetro $\zeta$ serve para definir o modelo a duas equações, a saber: no caso em que $\zeta=0$ o modelo considerado é o $\kappa-\varepsilon$ padrão de Launder e Spalding (1974), cujas constantes do modelo estão definidas na Tab. 4.1.

Tabela 4.1: Parâmetros do modelo $\kappa-\varepsilon$ padrão de turbulência.

\begin{tabular}{ccccc}
\hline \hline$C_{1 \varepsilon}$ & $C_{2 \varepsilon}$ & $C_{\mu}$ & $\sigma_{\kappa}$ & $\sigma_{\varepsilon}$ \\
\hline 1.44 & 1.92 & 0.09 & 1.0 & 1.3 \\
\hline \hline
\end{tabular}

No caso em que $\zeta=1$ o modelo de turbulência adotado é o $R N G \kappa-\varepsilon$ de Yakhot e Orszag (1986) onde as constantes estão definidas na Tab. 4.2.

Tabela 4.2: Parâmetros do modelo $R N G \kappa-\varepsilon$ de turbulência.

\begin{tabular}{ccccccc}
\hline \hline$C_{1 \varepsilon}$ & $C_{2 \varepsilon}$ & $C_{\mu}$ & $\sigma_{\kappa}$ & $\sigma_{\varepsilon}$ & $\eta_{0}$ & $\varsigma$ \\
\hline 1.42 & 1.68 & 0.085 & 0.7179 & 0.7179 & 4.38 & 0.015 \\
\hline \hline
\end{tabular}


As equações de trabalho consideradas nesta tese são as formas expandidas das Eqs. (4.28)-(4.31), juntamente com as definições (4.33)-(4.35), cujas formas adimensionais (sem o uso da notação * na adimensionalização e omitindo a notação de médias (־)) são apresentadas como segue:

$$
\begin{aligned}
& \frac{\partial u}{\partial t}=C O N V(u)-\frac{\partial p_{e}}{\partial x}+\frac{1}{R e}\left(\frac{\partial^{2} u}{\partial x^{2}}+\frac{\partial^{2} u}{\partial y^{2}}+\frac{\partial^{2} u}{\partial z^{2}}\right)+\frac{1}{F r^{2}} g_{x} \\
& +\frac{1}{R e}\left[2 \frac{\partial}{\partial x}\left(\nu_{t} \frac{\partial u}{\partial x}\right)+\frac{\partial}{\partial y}\left(\nu_{t}\left(\frac{\partial u}{\partial y}+\frac{\partial v}{\partial x}\right)\right)+\frac{\partial}{\partial z}\left(\nu_{t}\left(\frac{\partial u}{\partial z}+\frac{\partial w}{\partial x}\right)\right)\right], \\
& \frac{\partial v}{\partial t}=C O N V(v)-\frac{\partial p_{e}}{\partial y}+\frac{1}{R e}\left(\frac{\partial^{2} v}{\partial x^{2}}+\frac{\partial^{2} v}{\partial y^{2}}+\frac{\partial^{2} v}{\partial z^{2}}\right)+\frac{1}{F r^{2}} g_{y} \\
& +\frac{1}{R e}\left[2 \frac{\partial}{\partial y}\left(\nu_{t} \frac{\partial v}{\partial y}\right)+\frac{\partial}{\partial x}\left(\nu_{t}\left(\frac{\partial u}{\partial y}+\frac{\partial v}{\partial x}\right)\right)+\frac{\partial}{\partial z}\left(\nu_{t}\left(\frac{\partial v}{\partial z}+\frac{\partial w}{\partial y}\right)\right)\right], \\
& \frac{\partial w}{\partial t}=C O N V(w)-\frac{\partial p_{e}}{\partial z}+\frac{1}{R e}\left(\frac{\partial^{2} w}{\partial x^{2}}+\frac{\partial^{2} w}{\partial y^{2}}+\frac{\partial^{2} w}{\partial z^{2}}\right)+\frac{1}{F r^{2}} g_{z} \\
& +\frac{1}{R e}\left[2 \frac{\partial}{\partial z}\left(\nu_{t} \frac{\partial w}{\partial z}\right)+\frac{\partial}{\partial x}\left(\nu_{t}\left(\frac{\partial u}{\partial z}+\frac{\partial w}{\partial x}\right)\right)+\frac{\partial}{\partial y}\left(\nu_{t}\left(\frac{\partial v}{\partial z}+\frac{\partial w}{\partial y}\right)\right)\right], \\
& \frac{\partial u}{\partial x}+\frac{\partial v}{\partial y}+\frac{\partial w}{\partial z}=0 \\
& \frac{\partial \kappa}{\partial t}=C O N V(\kappa)+\frac{1}{R e}\left[\frac{\partial}{\partial x}\left(\left(1+\nu_{t} / \sigma_{\kappa}\right) \frac{\partial \kappa}{\partial x}\right)+\frac{\partial}{\partial y}\left(\left(1+\nu_{t} / \sigma_{\kappa}\right) \frac{\partial \kappa}{\partial y}\right)\right. \\
& \left.+\frac{\partial}{\partial z}\left(\left(1+\nu_{t} / \sigma_{\kappa}\right) \frac{\partial \kappa}{\partial z}\right)\right]+P-\varepsilon \\
& \frac{\partial \varepsilon}{\partial t}=C O N V(\varepsilon)+\frac{1}{R e}\left[\frac{\partial}{\partial x}\left(\left(1+\nu_{t} / \sigma_{\varepsilon}\right) \frac{\partial \varepsilon}{\partial x}\right)+\frac{\partial}{\partial y}\left(\left(1+\nu_{t} / \sigma_{\varepsilon}\right) \frac{\partial \varepsilon}{\partial y}\right)\right. \\
& \left.+\frac{\partial}{\partial z}\left(\left(1+\nu_{t} / \sigma_{\varepsilon}\right) \frac{\partial \varepsilon}{\partial z}\right)\right]+\left(C_{1 \varepsilon} P-C_{2 \varepsilon} \varepsilon\right) / T_{t}-\zeta \mathcal{R}, \\
& \nu_{t}=C_{\mu} \kappa T_{t} \\
& p_{e}=p+\frac{2}{3} \frac{1}{R e} \kappa \\
& P=\nu_{t}\left[2\left(\frac{\partial u}{\partial x}\right)^{2}+2\left(\frac{\partial v}{\partial y}\right)^{2}+2\left(\frac{\partial w}{\partial z}\right)^{2}+\left(\frac{\partial u}{\partial y}+\frac{\partial v}{\partial x}\right)^{2}\right. \\
& \left.+\left(\frac{\partial u}{\partial z}+\frac{\partial w}{\partial x}\right)^{2}+\left(\frac{\partial v}{\partial z}+\frac{\partial w}{\partial y}\right)^{2}\right]
\end{aligned}
$$




$$
\mathcal{R}=P \sqrt{\frac{P}{\nu_{t}}} \frac{\left(1-\eta / \eta_{0}\right)}{1+\varsigma \eta^{3}} \eta \frac{\varepsilon}{\kappa},
$$

onde $\eta$ é definido por

$$
\begin{aligned}
\eta & =\left[\left(\frac{\partial u}{\partial x}\right)^{2}+\left(\frac{\partial v}{\partial y}\right)^{2}+\left(\frac{\partial w}{\partial z}\right)^{2}+\frac{1}{2}\left(\frac{\partial u}{\partial y}+\frac{\partial v}{\partial x}\right)^{2}\right. \\
& \left.+\frac{1}{2}\left(\frac{\partial u}{\partial z}+\frac{\partial w}{\partial x}\right)^{2}+\frac{1}{2}\left(\frac{\partial v}{\partial z}+\frac{\partial w}{\partial y}\right)^{2}\right]^{\frac{1}{2}} \cdot \frac{\kappa}{\varepsilon}
\end{aligned}
$$

e $C O N V(\phi)$, com $\phi=u, v, w, \kappa$ ou $\varepsilon$, denota os termos convectivos dados por

$$
\begin{aligned}
& C O N V(u)=-\left(\frac{\partial(u u)}{\partial x}+\frac{\partial(u v)}{\partial y}+\frac{\partial(u w)}{\partial z}\right), \\
& \operatorname{CONV}(v)=-\left(\frac{\partial(v u)}{\partial x}+\frac{\partial(v v)}{\partial y}+\frac{\partial(v w)}{\partial z}\right), \\
& \operatorname{CONV}(w)=-\left(\frac{\partial(w u)}{\partial x}+\frac{\partial(w v)}{\partial y}+\frac{\partial(w w)}{\partial z}\right), \\
& \operatorname{CONV}(\kappa)=-\left(\frac{\partial(\kappa u)}{\partial x}+\frac{\partial(\kappa v)}{\partial y}+\frac{\partial(\kappa w)}{\partial z}\right), \\
& \operatorname{CONV}(\varepsilon)=-\left(\frac{\partial(\varepsilon u)}{\partial x}+\frac{\partial(\varepsilon v)}{\partial y}+\frac{\partial(\varepsilon w)}{\partial z}\right) .
\end{aligned}
$$

As equações de trabalho na forma adimensional e em um sistema de coordenadas gerais são dadas por

$$
\begin{gathered}
\frac{\partial \mathbf{u}}{\partial t}+\nabla \cdot(\mathbf{u u})=-\nabla p_{e}+\frac{1}{R e} \nabla^{2} \mathbf{u}+\frac{1}{F r^{2}} \mathbf{g}+\frac{1}{R e} \nabla \cdot\left(\nu_{t} \mathbf{D}\right), \\
\nabla \cdot \mathbf{u}=0, \\
\frac{\partial \kappa}{\partial t}+\nabla \cdot(\kappa \mathbf{u})=\frac{1}{R e} \nabla \cdot\left(\left(1+\frac{\nu_{t}}{\sigma_{\kappa}}\right) \nabla \kappa\right)+P-\varepsilon, \\
\frac{\partial \varepsilon}{\partial t}+\nabla \cdot(\varepsilon \mathbf{u})=\frac{1}{R e} \nabla \cdot\left(\left(1+\frac{\nu_{t}}{\sigma_{\varepsilon}}\right) \nabla \varepsilon\right)+\left(C_{1 \varepsilon} P-C_{2 \varepsilon} \varepsilon\right) / T_{t}-\zeta \mathcal{R}, \\
P=\nu_{t}(\mathbf{D}: \nabla \mathbf{u}) .
\end{gathered}
$$

\subsubsection{Formulação matemática para fluidos não newtonianos}

As equações (4.1) e (4.2), mais o tensor de tensões $\boldsymbol{\sigma}$ dado pela Eq. (4.5), definem a modelagem matemática para estudar escoamentos de fluidos não newtonianos. Neste trabalho, o modelo viscoelástico escolhido foi o Single eXtended Pom Pom (SXPP) de Verbeeten et al. (2002). Nessa modelagem, o tensor do polímero $\boldsymbol{\tau}_{p}$ é determinado usando-se a equação (constitutiva) característica do modelo definida por

$$
f \boldsymbol{\tau}_{p}+\lambda_{1} \stackrel{\nabla}{\boldsymbol{\tau}_{p}}+G_{0}(f-1) \mathbb{I}+\frac{\varrho}{G_{0}}\left(\boldsymbol{\tau}_{p} \cdot \boldsymbol{\tau}_{p}\right)=2 \mu_{p} \mathbf{D},
$$


onde $f=f\left(\lambda, \boldsymbol{\tau}_{p}\right)$ é uma variável auxiliar calculada como

$$
f=2 \frac{1}{\gamma}\left(1-\frac{1}{\lambda}\right) e^{Q_{0}(\lambda-1)}+\frac{1}{\lambda^{2}}\left[1-\frac{\varrho}{3 G_{0}}\left|\operatorname{tr}\left(\boldsymbol{\tau}_{p} \cdot \boldsymbol{\tau}_{p}\right)\right|\right],
$$

sendo $\lambda=\lambda\left(\boldsymbol{\tau}_{p}\right)$, um parâmetro relacionado com a molécula polimérica (Figueiredo, 2011), dado por

$$
\lambda=\sqrt{1+\frac{1}{3 G_{0}}\left|\operatorname{tr}\left(\boldsymbol{\tau}_{p}\right)\right|}
$$

e $\stackrel{\nabla}{\boldsymbol{\tau}_{p}}$ a derivada contravariante de $\boldsymbol{\tau}_{p}$ dada por

$$
{\stackrel{\nabla}{\boldsymbol{\tau}_{p}}}=\frac{\partial \boldsymbol{\tau}_{p}}{\partial t}+\nabla \cdot\left(\mathbf{u} \boldsymbol{\tau}_{p}\right)-(\nabla \mathbf{u}) \cdot \boldsymbol{\tau}_{p}-\boldsymbol{\tau}_{p} \cdot \nabla(\mathbf{u})^{T}
$$

Nas Eqs. (4.58) e (4.59) $G_{0}$ é o módulo de relaxação linear; $Q_{0}$ é um parâmetro calculado a partir do número de ramificações $Q$ na extremidade da molécula polimérica, tal que $Q Q_{0}=2$ (Movagar et al., 2010, Figueiredo, 2011); e $\varrho$ é um parâmetro relacionado à anisotropia do material. A viscosidade do polímero $\mu_{p}$ é proporcional à $\lambda_{1}$ e usada neste trabalho como $\mu_{p}=G_{0} \lambda_{1}$.

As equações de trabalho em coordenadas cartesianas (na forma expandida adimensional) para a simulação computacional de escoamentos incompressíveis de fluidos não newtonianos são dadas por

$$
\begin{aligned}
& \frac{\partial u}{\partial t}- C O N V(u)= \\
&-\frac{\partial p}{\partial x}+\frac{\vartheta}{R e}\left(\frac{\partial^{2} u}{\partial x^{2}}+\frac{\partial^{2} u}{\partial y^{2}}+\frac{\partial^{2} u}{\partial z^{2}}\right)+\frac{\partial \tau_{p_{x x}}}{\partial x}+\frac{\partial \tau_{p_{x y}}}{\partial y}+\frac{\partial \tau_{p_{x z}}}{\partial z}+\frac{1}{F r^{2}} g_{x} \\
& \frac{\partial v}{\partial t}- C O N V(v)= \\
&-\frac{\partial p}{\partial y}+\frac{\vartheta}{R e}\left(\frac{\partial^{2} v}{\partial x^{2}}+\frac{\partial^{2} v}{\partial y^{2}}+\frac{\partial^{2} v}{\partial z^{2}}\right)+\frac{\partial \tau_{p_{y x}}}{\partial x}+\frac{\partial \tau_{p_{y y}}}{\partial y}+\frac{\partial \tau_{p_{y z}}}{\partial z}+\frac{1}{F r^{2}} g_{y} \\
& \frac{\partial w}{\partial t}- C O N V(w)= \\
&-\frac{\partial p}{\partial z}+\frac{\vartheta}{R e}\left(\frac{\partial^{2} w}{\partial x^{2}}+\frac{\partial^{2} w}{\partial y^{2}}+\frac{\partial^{2} w}{\partial z^{2}}\right)+\frac{\partial \tau_{p_{z x}}}{\partial x}+\frac{\partial \tau_{p_{z y}}}{\partial y}+\frac{\partial \tau_{p_{z z}}}{\partial z}+\frac{1}{F r^{2}} g_{z} \\
& \frac{\partial u}{\partial x}+\frac{\partial v}{\partial y}+\frac{\partial w}{\partial z}=0 \\
& \frac{\partial \tau_{p_{x x}}}{\partial t}= \\
& 2 \Upsilon \frac{\partial u}{\partial x}+2\left(\frac{\partial u}{\partial x} \tau_{p_{x x}}+\frac{\partial u}{\partial y} \tau_{p_{x y}}+\frac{\partial u}{\partial z} \tau_{p_{x z}}\right)+C O N V\left(\tau_{p_{x x}}\right) \\
& \frac{1}{W e}\left\{f \tau_{p_{x x}}+\Upsilon(f-1)+\frac{\varrho}{\Upsilon}\left[\left(\tau_{p_{x x}}\right)^{2}+\left(\tau_{p_{x y}}\right)^{2}+\left(\tau_{p_{x z}}\right)^{2}\right]\right\}
\end{aligned}
$$




$$
\begin{aligned}
& \frac{\partial \tau_{p_{y y}}}{\partial t}=2 \Upsilon \frac{\partial v}{\partial y}+2\left(\frac{\partial v}{\partial x} \tau_{p_{x y}}+\frac{\partial v}{\partial y} \tau_{p_{y y}}+\frac{\partial v}{\partial z} \tau_{p_{y z}}\right)+\operatorname{CONV}\left(\tau_{p_{y y}}\right) \\
& -\frac{1}{W e}\left\{f \tau_{p_{y y}}+\Upsilon(f-1)+\frac{\varrho}{\Upsilon}\left[\left(\tau_{p_{x y}}\right)^{2}+\left(\tau_{p_{y y}}\right)^{2}+\left(\tau_{p_{y z}}\right)^{2}\right]\right\}, \\
& \frac{\partial \tau_{p_{z z}}}{\partial t}=2 \Upsilon \frac{\partial w}{\partial z}+2\left(\frac{\partial w}{\partial x} \tau_{p_{x z}}+\frac{\partial w}{\partial y} \tau_{p_{y z}}+\frac{\partial w}{\partial z} \tau_{p_{z z}}\right)+\operatorname{CONV}\left(\tau_{p_{z z}}\right) \\
& -\frac{1}{W e}\left\{f \tau_{p_{z z}}+\Upsilon(f-1)+\frac{\varrho}{\Upsilon}\left[\left(\tau_{p_{x z}}\right)^{2}+\left(\tau_{p_{y z}}\right)^{2}+\left(\tau_{p_{z z}}\right)^{2}\right]\right\}, \\
& \frac{\partial \tau_{p_{x y}}}{\partial t}=\Upsilon\left(\frac{\partial u}{\partial y}+\frac{\partial v}{\partial x}\right)+\frac{\partial u}{\partial x} \tau_{p_{x y}}+\frac{\partial u}{\partial y} \tau_{p_{y y}}+\frac{\partial u}{\partial z} \tau_{p_{y z}}+\frac{\partial v}{\partial x} \tau_{p_{x x}}+\frac{\partial v}{\partial y} \tau_{p_{x y}}+\frac{\partial v}{\partial z} \tau_{p_{x z}} \\
& +C O N V\left(\tau_{p_{x y}}\right)-\frac{1}{W e}\left\{f \tau_{p_{x y}}+\frac{\varrho}{\Upsilon}\left[\tau_{p_{x x}} \tau_{p_{x y}}+\tau_{p_{x y}} \tau_{p_{y y}}+\tau_{p_{x z}} \tau_{p_{y z}}\right]\right\}, \\
& \frac{\partial \tau_{p_{x z}}}{\partial t}=\Upsilon\left(\frac{\partial u}{\partial z}+\frac{\partial w}{\partial x}\right)+\frac{\partial u}{\partial x} \tau_{p_{x z}}+\frac{\partial u}{\partial y} \tau_{p_{y z}}+\frac{\partial u}{\partial z} \tau_{p_{z z}}+\frac{\partial w}{\partial x} \tau_{p_{x x}}+\frac{\partial w}{\partial y} \tau_{p_{x y}}+\frac{\partial w}{\partial z} \tau_{p_{x z}} \\
& +\operatorname{CONV}\left(\tau_{p_{x z}}\right)-\frac{1}{W e}\left\{f \tau_{p_{x z}}+\frac{\varrho}{\Upsilon}\left[\tau_{p_{x x}} \tau_{p_{x z}}+\tau_{p_{x y}} \tau_{p_{y z}}+\tau_{p_{x z}} \tau_{p_{z z}}\right]\right\}, \\
& \frac{\partial \tau_{p_{y z}}}{\partial t}=\Upsilon\left(\frac{\partial v}{\partial z}+\frac{\partial w}{\partial y}\right)+\frac{\partial v}{\partial x} \tau_{p_{x z}}+\frac{\partial v}{\partial y} \tau_{p_{y z}}+\frac{\partial v}{\partial z} \tau_{p_{z z}}+\frac{\partial w}{\partial x} \tau_{p_{x y}}+\frac{\partial w}{\partial y} \tau_{p_{y y}}+\frac{\partial w}{\partial z} \tau_{p_{y z}} \\
& +\operatorname{CONV}\left(\tau_{p_{y z}}\right)-\frac{1}{W e}\left\{f \tau_{p_{y z}}+\frac{\varrho}{\Upsilon}\left[\tau_{p_{x y}} \tau_{p_{x z}}+\tau_{p_{y y}} \tau_{p_{y z}}+\tau_{p_{y z}} \tau_{p_{z z}}\right]\right\}
\end{aligned}
$$

em que $\Upsilon=\frac{1-\vartheta}{R e W e} \operatorname{eONV}(\phi)$, com $\phi=\tau_{p_{x x}}, \tau_{p_{y y}}, \tau_{p_{z z}}, \tau_{p_{x y}}, \tau_{p_{x z}}$ ou $\tau_{p_{y z}}$, denota os termos convectivos dados por

$$
\begin{aligned}
& \operatorname{CONV}\left(\tau_{p_{x x}}\right)=-\left(\frac{\partial\left(u \tau_{p_{x x}}\right)}{\partial x}+\frac{\partial\left(v \tau_{p_{x x}}\right)}{\partial y}+\frac{\partial\left(w \tau_{p_{x x}}\right)}{\partial z}\right), \\
& \operatorname{CONV}\left(\tau_{p_{y y}}\right)=-\left(\frac{\partial\left(u \tau_{p_{y y}}\right)}{\partial x}+\frac{\partial\left(v \tau_{p_{y y}}\right)}{\partial y}+\frac{\partial\left(w \tau_{p_{y y}}\right)}{\partial z}\right), \\
& \operatorname{CONV}\left(\tau_{p_{z z}}\right)=-\left(\frac{\partial\left(u \tau_{p_{z z}}\right)}{\partial x}+\frac{\partial\left(v \tau_{p_{z z}}\right)}{\partial y}+\frac{\partial\left(w \tau_{p_{z z}}\right)}{\partial z}\right), \\
& \operatorname{CONV}\left(\tau_{p_{x y}}\right)=-\left(\frac{\partial\left(u \tau_{p_{x y}}\right)}{\partial x}+\frac{\partial\left(v \tau_{p_{x y}}\right)}{\partial y}+\frac{\partial\left(w \tau_{p_{x y}}\right)}{\partial z}\right), \\
& \operatorname{CONV}\left(\tau_{p_{x z}}\right)=-\left(\frac{\partial\left(u \tau_{p_{x z}}\right)}{\partial x}+\frac{\partial\left(v \tau_{p_{x z}}\right)}{\partial y}+\frac{\partial\left(w \tau_{p_{x z}}\right)}{\partial z}\right), \\
& \operatorname{CONV}\left(\tau_{p_{y z}}\right)=-\left(\frac{\partial\left(u \tau_{p_{y z}}\right)}{\partial x}+\frac{\partial\left(v \tau_{p_{y z}}\right)}{\partial y}+\frac{\partial\left(w \tau_{p_{y z}}\right)}{\partial z}\right) .
\end{aligned}
$$


Em um sistema de coordenadas gerais as equações de trabalho (na forma adimensional) para a simulação computacional de escoamentos incompressíveis de fluidos não newtonianos são dadas por

$$
\begin{gathered}
\frac{\partial \mathbf{u}}{\partial t}+\nabla \cdot(\mathbf{u u})=\nabla \cdot \boldsymbol{\sigma}+\frac{1}{F r^{2}} \mathbf{g} \\
\nabla \cdot \mathbf{u}=0 \\
\boldsymbol{\sigma}=-p \mathbb{I}+\frac{2 \vartheta}{R e} \mathbf{D}+\boldsymbol{\tau}_{p}, \\
f \boldsymbol{\tau}_{p}+W e \frac{\nabla}{\boldsymbol{\tau}_{p}}+\Upsilon(f-1) \mathbb{I}+\varrho \frac{1}{\Upsilon} \boldsymbol{\tau}_{p} \cdot \boldsymbol{\tau}_{p}=2 \frac{1-\vartheta}{R e} \mathbf{D}, \\
f=\frac{2}{\gamma}\left(1-\frac{1}{\lambda}\right) e^{Q_{0}(\lambda-1)}+\frac{1}{\lambda^{2}}\left[1-\frac{\varrho}{3}\left(\frac{1}{\Upsilon}\right)^{2} \operatorname{tr}\left(\boldsymbol{\tau}_{p} \cdot \boldsymbol{\tau}_{p}\right)\right] \\
\lambda=\sqrt{1+\frac{1}{3 \Upsilon}\left|t r\left(\boldsymbol{\tau}_{p}\right)\right|} \\
\Upsilon=\frac{1-\vartheta}{R e W e}, \\
\boldsymbol{\tau}_{p}=\frac{\partial \boldsymbol{\tau}_{p}}{\partial t}+\nabla \cdot\left(\mathbf{u} \boldsymbol{\tau}_{p}\right)-(\nabla \mathbf{u}) \cdot \boldsymbol{\tau}_{p}-\boldsymbol{\tau}_{p} \cdot \nabla(\mathbf{u})^{T} .
\end{gathered}
$$

\subsection{Metodologia de solução}

A metodologia computacional básica para simular escoamentos incompressíveis com superfícies livres móveis é o método em variáveis primitivas Marker-And-Cell (MAC, Los Alamos); o método é uma variante do método de projeção de Chorin (1968) descrito por Harlow e Welch (1965) (McKee et al. (2008) também fornecem uma revisão ampla do método). O método é no contexto de diferenças finitas e baseado numa malha euleriana deslocada (ou diferenciada) em que a posição do fluido no domínio é determinada usando partículas marcadoras virtuais (sem massa, sem volume ou quaisquer outras propriedades). Essas partículas são utilizadas, fundamentalmente, para indicar a configuração do fluido no domínio de solução, mostrando regiões ocupadas por fluido e regiões vazias; a cada passo no tempo, as partículas marcadoras são deslocadas para novas posições usando velocidades locais do fluido obtidas via equações de Navier-Stokes. A metodologia escolhida nesse trabalho é a Simplified MAC (SMAC) de Amsden e Harlow (1970), cujo objetivo é superar as deficiências do método MAC. Em suma, o método SMAC calcula um campo provisório de velocidades e depois uma correção deste empregando uma função potencial (auxiliar) para satisfazer a condição de incompressibilidade.

Na malha deslocada as variáveis escalares, tais como pressão $p, \kappa, \varepsilon, \nu_{t}$ e os componentes do tensor $\boldsymbol{\tau}_{p}$, são avaliadas no centro de uma célula computacional, ao passo que os componentes $u, v$ e $w$ do vetor velocidade $\mathbf{u}$ são posicionados nas faces $i+\frac{1}{2}, j+\frac{1}{2}$ e $k+\frac{1}{2}$, respectivamente. Uma célula computacional 3D típica envolvida nos cálculos está mostrada na Fig. 4.1.

As partículas de fluido são avaliadas sobre uma malha euleriana uniforme, em que cada célula computacional, a cada passo no tempo, é classificada de acordo com sua posição relativa ao fluido, a contornos rígidos e interfaces; células com mais da metade de seu volume dentro do domínio computa- 


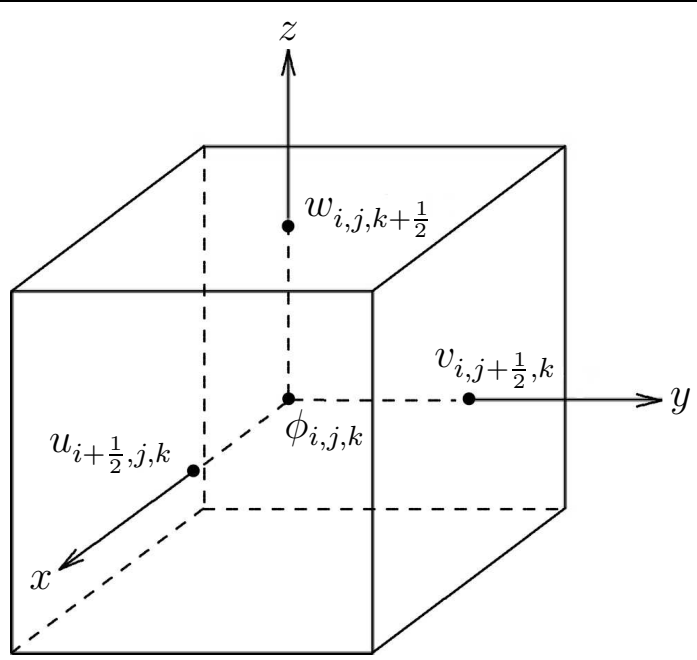

Figura 4.1: Célula computacional da malha deslocada. A variável $\phi$ representa $p_{e}, \kappa, \varepsilon, \nu_{t}, \tau_{p_{x x}}, \tau_{p_{x y}}$, $\tau_{p_{x z}}, \tau_{p_{y y}}, \tau_{p_{y z}}, \tau_{p_{x x}}$ e $\tau_{p_{z z}}$.

cional são classificadas como células Boundary (B); o mesmo critério é empregado para definir células Inflow (I). Qualquer célula computacional inteiramente contida no fluido é classificada como $\mathbf{F}$ ull (F). Aquelas completamente fora do fluido (mas dentro do domínio computacional) são classificadas como células $\mathbf{E}$ mpty $(\mathbf{E})$. Células contendo partículas marcadoras e tendo como vizinho ao menos uma célula E são classificadas como células $\mathbf{S}$ urface (S) (McKee et al., 2008). A Fig. 4.2 ilustra um exemplo no caso de uma configuração de células computacionais no interior de um domínio 2D, mostrando os vários tipos de células.

\subsubsection{Método de projeção e algoritmo computacional}

Para a simulação computacional de escoamentos viscosos, transientes, incompressíveis e newtonianos, envolvendo superfícies livre móveis e modelados pelas equações de trabalho (4.53)-(4.56), emprega-se neste trabalho o método de projeção de Chorin (1968).

Em resumo, o método é como segue: num primeiro estágio dos cálculos, um campo de velocidades

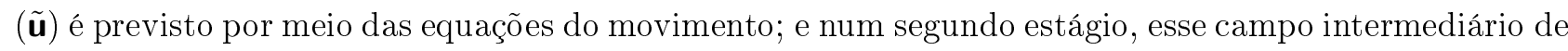
velocidades e o campo de pressão são corrigidos com o propósito de satisfazer a equação de conservação de massa. Neste segundo estágio se deriva uma equação de Poisson para um campo projetor (ou um potencial auxiliar) $\psi=\psi(\mathbf{x}, t)$, com condições de contorno tipo Neumann. O método de solução proposto a seguir ( formulação explícita) é para resolver problemas em regime laminar ou turbulentos, em que se introduz o parâmetro $\alpha$ na equação de movimento (4.53) para, simplesmente, "ligar" $(\alpha=1)$ ou "desligar" $(\alpha=0)$ a modelagem de turbulência $(\kappa-\varepsilon$ padrão ou $R N G \kappa-\varepsilon)$.

Considera-se $\tilde{p_{e}}$ uma pressão qualquer que satisfaz condições de contorno adequadas na superfície livre do fluido: nas células $\mathbf{S}$ impõem-se tensões normais nulas; e nas células $\mathbf{F}$ prescreve-se a pressão efetiva como $\tilde{p_{e}}=0$. A partir da Eq. (4.53), define-se a relação

$$
\tilde{\mathbf{u}}=\mathbf{u}^{n}+\delta t\left\{-\nabla \cdot(\mathbf{u u})^{n}-\nabla \tilde{p}_{e}^{n}+\operatorname{Re}^{-1} \nabla^{2} \mathbf{u}^{n}+\left(F r^{2}\right)^{-1} \boldsymbol{g}^{n}+\alpha R e^{-1} \nabla \cdot\left(\nu_{t} \mathbf{D}\right)^{n}\right\}
$$




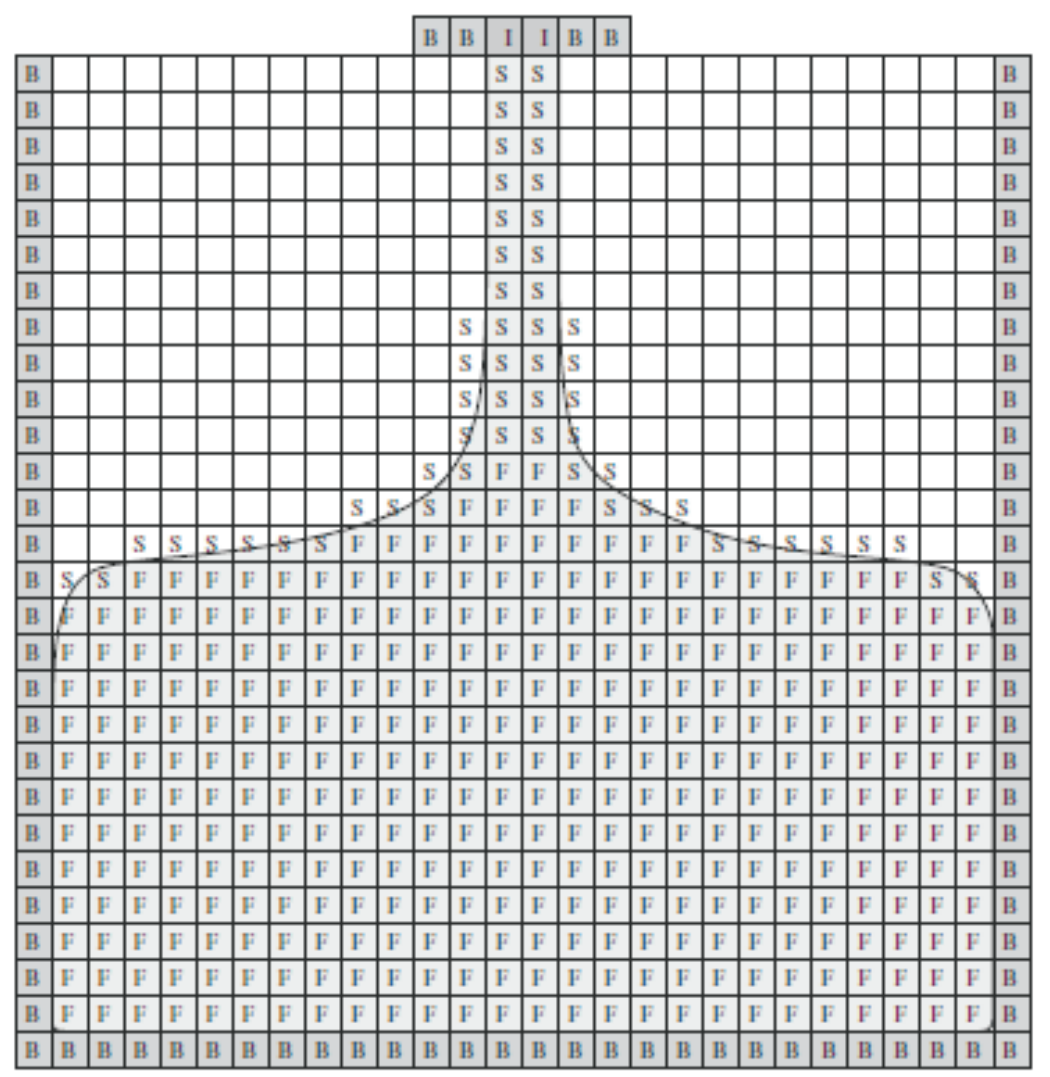

Figura 4.2: Estrutura celular. Tomado de Ferreira et al. (2007).

em que ${\tilde{p_{e}}}^{n}$ é uma pressão efetiva tentativa e aproximada por $p_{e}{ }^{n+1}$. O campo intermediário da velocidade $\tilde{\mathbf{u}}$ pode ser decomposto usando-se o teorema de Helmholtz-Hodge (Hodge (1952)) como

$$
\tilde{\mathbf{u}}=\mathbf{u}^{n+1}+\nabla \psi^{n+1} \text {. }
$$

Forçando $\mathbf{u}^{n+1}$ satisfazer $\nabla \cdot \mathbf{u}^{n+1}=0$ e aplicando o divergente em (4.87), obtém-se a equação de Poisson para o potencial $\psi$

$$
\nabla^{2} \psi^{n+1}=\nabla \cdot \tilde{\mathbf{u}}
$$

Substituindo (4.87) em (4.86), tem-se

$$
\frac{\mathbf{u}^{n+1}+\nabla \psi^{n+1}}{\delta t}=\frac{\mathbf{u}^{n}}{\delta t}-\nabla \cdot(\mathbf{u u})^{n}-\nabla \tilde{p}_{e}^{n}+R e^{-1} \nabla^{2} \mathbf{u}^{n}+\left(F r^{2}\right)^{-1} \boldsymbol{g}^{n}+\alpha R e^{-1} \nabla \cdot\left(\nu_{t} \mathbf{D}\right)^{n}
$$

A pressão efetiva é obtida comparando-se (4.89) e (4.86) e o resultado é

$$
p_{e}^{n+1}=p_{e}^{n}+\frac{\psi^{n+1}}{\delta t} .
$$


Os passos adotados no algoritmo computacional para a simulação dos efeitos da turbulência $(\alpha=1)$ usando o método de projeção é como segue. Admite-se que num dado tempo $t_{0}$ as variáveis dependentes são conhecidas em todo domínio de cálculo e as condições de fronteira estão especificadas. Um ciclo computacional requer atualizar as variáveis discretas no tempo $t_{0}+\delta t$ utilizando-se os seguintes passos:

(1) Usando uma discretização explícita e com a viscosidade turbulenta conhecida, calcula-se o campo de velocidades intermediário $\tilde{\mathbf{u}}$ a partir de $(4.86), \operatorname{com} \tilde{\mathbf{u}}\left(\mathbf{x}, t_{0}\right)=\mathbf{u}\left(\mathbf{x}, t_{0}\right)$;

(2) Resolve-se a equação de Poisson (4.88) com as condições de Dirichlet homogêneas na superfície livre e saída de fluido, e de Neumann em contornos rígidos e entrada de fluido;

(3) Corrige-se o campo de velocidade por meio de (4.87);

(4) Atualiza-se o campo de pressão usando a expressão (4.90);

(5) Calcula-se a energia cinética turbulenta $\kappa$ por (4.55);

(6) Calcula-se a dissipação da energia $\varepsilon$ por (4.56);

(7) Atualiza-se a viscosidade turbulenta $\nu_{t}$ via (4.43);

(8) Determinam-se as posições das partículas marcadoras resolvendo a EDO

$$
\frac{d \mathbf{x}}{d t}=\mathbf{u}^{n+1} \quad \text { por Euler explícito. }
$$

(9) Atualizam-se as condições de contorno para dar início ao próximo ciclo e retorna-se ao passo (1).

Para o caso da simulação de escoamentos de fluidos newtonianos incompressíveis no regime laminar, em que a constante $\alpha$ assume o valor nulo, usa-se o mesmo algoritmo descrito anteriormente para $\alpha=1$ e eliminam-se os passos (5, 6 e 7 .

A formulação implícita usando o método de Crank-Nicolson para resolver escoamentos incompressíveis não newtonianos e com superfícies livres mais o algoritmo computacional de solução são descritos como segue. Inicialmente, para simplificar, fazendo-se o uso da definição da derivada contravariante (4.85), reescreve-se a Eq. (4.81) na seguinte forma

$$
\begin{aligned}
& \frac{\partial \boldsymbol{\tau}_{p}}{\partial t}=2 \Upsilon \mathbf{D}-\left\{\nabla \cdot\left(\mathbf{u} \boldsymbol{\tau}_{p}\right)-(\nabla \mathbf{u}) \cdot \boldsymbol{\tau}_{p}-\boldsymbol{\tau}_{p} \cdot(\nabla \mathbf{u})^{T}\right\}- \\
& \frac{1}{W e}\left\{f \boldsymbol{\tau}_{p}+\Upsilon(f-1) \mathbb{I}+\varrho \frac{1}{\Upsilon} \boldsymbol{\tau}_{p} \cdot \boldsymbol{\tau}_{p}\right\}=F=F\left(\mathbf{u}, \boldsymbol{\tau}_{p}\right) .
\end{aligned}
$$

As Eqs. (4.78) e (4.79) são também rearranjadas como

$$
\begin{aligned}
& \frac{\mathbf{u}^{(n+1)}-\mathbf{u}^{(n)}}{\delta t}+\nabla \cdot(\mathbf{u u})^{(n)}+\nabla p^{(n+1)}=\frac{\vartheta}{2 R e}\left[\nabla^{2} \mathbf{u}^{(n)}+\nabla^{2} \mathbf{u}^{(n+1)}\right]+\nabla \cdot \boldsymbol{\tau}_{p}^{\left(n+\frac{1}{2}\right)}+\frac{1}{F r^{2}} \mathbf{g}^{(n)}, \\
& \nabla \mathbf{u}^{(n+1)}=0,
\end{aligned}
$$


onde

$$
\nabla \cdot \boldsymbol{\tau}_{p}^{\left(n+\frac{1}{2}\right)}=\frac{1}{2}\left(\nabla \cdot \tilde{\boldsymbol{\tau}}_{p}^{(n+1)}+\nabla \cdot \boldsymbol{\tau}_{p}^{(n)}\right)
$$

sendo $\tilde{\boldsymbol{\tau}}_{p}^{(n+1)}$ uma previsão para o tensor $\boldsymbol{\tau}_{p}$ em (4.92). Usando-se o método de Runge-Kutta explícito de segunda ordem essa previsão é obtida por meio de

$$
\frac{\tilde{\boldsymbol{\tau}}_{p}^{n+1}-\boldsymbol{\tau}_{p}^{n}}{\delta t}=F\left(\mathbf{u}, \boldsymbol{\tau}_{p}\right)
$$

e calcula-se $\boldsymbol{\tau}_{p}{ }^{n+1}$ por

$$
\frac{\boldsymbol{\tau}_{p}^{n+1}-\boldsymbol{\tau}_{p}^{n}}{\delta t}=\frac{1}{2}\left[F\left(\mathbf{u}^{(n)}, \boldsymbol{\tau}_{p}^{(n)}\right)+F\left(\mathbf{u}^{(n+1)}, \tilde{\boldsymbol{\tau}}_{p}^{(n+1)}\right)\right]
$$

O campo intermediário ũ é obtido por meio de

$$
\frac{\tilde{\mathbf{u}}^{(n+1)}-\tilde{\mathbf{u}}^{(n)}}{\delta t}+\nabla \cdot(\mathbf{u u})^{(n)}+\nabla \tilde{p}^{(n+1)}=\frac{\vartheta}{2 R e}\left[\nabla^{2} \tilde{\mathbf{u}}^{(n)}+\nabla^{2} \tilde{\mathbf{u}}^{(n+1)}\right]+\nabla \cdot \boldsymbol{\tau}_{p}^{\left(n+\frac{1}{2}\right)}+\frac{1}{F r^{2}} \mathbf{g}^{(n)},
$$

onde $\tilde{\mathbf{u}}^{(n)}=\mathbf{u}^{(n)}$ e $\tilde{p}^{(n+1)}=p^{(n)}$. A pressão $p$ é atualizada por

$$
p^{(n+1)}=\tilde{p}^{(n+1)}+\frac{\psi^{(n+1)}}{\delta t}-\frac{\vartheta}{2 R e} \nabla^{2} \psi^{(n+1)},
$$

$\operatorname{com} \psi^{(n+1)}$ obtido pela Eq. (4.88).

O procedimento de solução adotado no caso da simulação de escoamentos viscoelásticos é similar ao descrito anteriormente para escoamentos de fluidos newtonianos (médodo da projeção); com mudanças nos cálculos do campo intermediário de velocidade ũ e na correção da pressão $p$, e com a inclusão do cálculo do tensor do polímero $\boldsymbol{\tau}_{p}$. Os detalhes são descritos a seguir. Admite-se que num dado nível de tempo $n$ as variáveis dependentes (incluindo o tensor do polímero) são conhecidas e as condições de contorno estão especificadas. Um ciclo computacional requer atualizar as variáveis discretas no nível de tempo $n+1$ utilizando-se os seguintes passos:

(1) Calcula-se $\tilde{\boldsymbol{\tau}}_{p}^{(n+1)}$ por meio da Eq. (4.96);

(2) Calcula-se o campo de velocidades ũ por meio da Eq. (4.98);

(3) Resolva-se a equação de Poisson (4.88) com as condições apropriadas;

(4) Atualiza-se o campo de velocidade por (4.87);

(5) Atualiza-se o campo de pressão por meio de (4.99);

(6) Calcula-se $\boldsymbol{\tau}_{p}{ }^{(n+1)}$ via Eq. (4.97);

(7) Calcula-se a posição das partículas marcadoras resolvendo a EDO (4.91);

(8) Atualizam-se as condições de contorno para dar início ao próximo ciclo e retorna-se ao passo (1). 
Neste ponto é importante destacar que a implementação dos termos convectivos (não lineares) que aparecem nas equações (4.48)-(4.52) e (4.72)-(4.77) é feita usando-se o novo esquema interpolativo upwind TDPUS-C3.

\subsection{Equações de trabalho discretas}

Nessa seção, apresentam-se as discretizações das equações de trabalho para problemas de escoamentos de fluidos viscosos. Todas as derivadas temporais, com exceção daquelas na equação da quantidade de movimento (4.62)-(4.64) em que são aproximadas pela técnica de Crank-Nicolson, são aproximadas pelo método de Euler explícito. Para a marcha no tempo, é utilizada a restrição (de estabilidade) para o passo temporal

$$
\delta t=\chi \cdot \min \left(\delta t_{\text {viscoso }}, \delta_{C F L}\right),
$$

em que $\chi$ é uma constante no intervalo $(0,1]$, introduzida para assegurar que os cálculos permaneçam limitados, com $\delta t_{v i s c o s o}$ e $\delta_{C F L}$ definidos, respectivamente, pelas seguintes expressões:

$$
\delta t_{\text {viscoso }} \leq \varkappa \frac{\operatorname{Re}}{2}\left(\frac{1}{(\delta x)^{2}}+\frac{1}{(\delta y)^{2}}+\frac{1}{(\delta z)^{2}}\right)^{-1},
$$

onde $\varkappa=1$ se o problema em questão é newtoniano (em regime turbulento ou não), e $\varkappa=\vartheta$, dado em (4.10), se o problema é viscoelástico; e

$$
\delta_{C F L} \leq \min \left\{\delta t_{u}, \delta t_{v}, \delta t_{w}\right\}
$$

sendo

$$
\delta t_{u} \leq\left(\frac{\delta x}{\max \left\{|u|^{n}\right\}}\right), \quad \delta t_{v} \leq\left(\frac{\delta y}{\max \left\{|v|^{n}\right\}}\right), \quad \delta t_{w} \leq\left(\frac{\delta z}{\max \left\{|w|^{n}\right\}}\right) .
$$

As quantidades $\max \left\{|u|^{n}\right\}, \max \left\{|v|^{n}\right\}$ e $\max \left\{|w|^{n}\right\}$ correspondem aos valores máximos, em valor absoluto, das velocidades $u, v, w$ em cada ciclo computacional, respectivamente.

Com exceção das derivadas que compõem os termos convectivos (não lineares), os quais são aproximados neste trabalho pelo esquema TDPUS-C3 (ver detalhes adiante), todas as demais derivadas são discretizadas usando diferenças centrais de segunda ordem de precisão local. Em particular, os termos que advém da modelagem da turbulência nas Eqs. (4.37)-(4.39) e (4.41)-(4.42) e que contemplam a viscosidade turbulenta $\nu_{t}$ são aproximados como está feito nos exemplos que segue. O segundo termo na Eq. (4.38) avaliado no ponto $\mathcal{P}=\left(i, j+\frac{1}{2}, k\right)$ é aproximado por

$$
\begin{aligned}
\left.\frac{\partial}{\partial x}\left(\nu_{t}\left(\frac{\partial u}{\partial y}+\frac{\partial v}{\partial x}\right)\right)\right|_{i, j+\frac{1}{2}, k} ^{n} & \approx \frac{1}{\delta x}\left[\left.\left(\nu_{t}\left(\frac{\partial u}{\partial y}+\frac{\partial v}{\partial x}\right)\right)\right|_{i+\frac{1}{2}, j+\frac{1}{2}, k} ^{n}-\left.\left(\nu_{t}\left(\frac{\partial u}{\partial y}+\frac{\partial v}{\partial x}\right)\right)\right|_{i-\frac{1}{2}, j+\frac{1}{2}, k} ^{n}\right] \\
& \frac{\partial}{\partial x}\left[\nu_{t_{i+\frac{1}{2}, j+\frac{1}{2}, k}}^{n}\left(\frac{u_{i+\frac{1}{2}, j+1, k}-u_{i+\frac{1}{2}, j-1, k}}{\delta y}+\frac{v_{i+1, j+\frac{1}{2}, k}-v_{i, j+\frac{1}{2}, k}}{\delta x}\right)\right. \\
& \left.\nu_{t_{i-\frac{1}{2}, j+\frac{1}{2}, k}^{n}}\left(\frac{u_{i-\frac{1}{2}, j, k}-u_{i-\frac{1}{2}, j-1, k}}{\delta y}+\frac{v_{i, j+\frac{1}{2}, k}-v_{i-1, j+\frac{1}{2}, k}}{\delta x}\right)\right],
\end{aligned}
$$


onde

$$
\begin{aligned}
& \nu_{t_{i+\frac{1}{2}, j+\frac{1}{2}, k}}^{n} \approx 0.25\left(\nu_{t_{i+1, j+1, k}}^{n}+\nu_{t_{i+1, j, k}}^{n}+\nu_{t_{i, j+1, k}}^{n}+\nu_{t_{i, j, k}}^{n}\right) \quad \mathrm{e} \\
& \nu_{t_{i-\frac{1}{2}, j+\frac{1}{2}, k}}^{n} \approx 0.25\left(\nu_{t_{i, j+1, k}}^{n}+\nu_{t_{i, j, k}}^{n}+\nu_{t_{i-1, j+1, k}}^{n}+\nu_{t_{i-1, j, k}}^{n}\right) .
\end{aligned}
$$

O segundo termo na Eq. (4.41) avaliado na posição $\mathcal{P}=(i, j, k)$ é aproximado por

$$
\begin{aligned}
\left.\frac{\partial}{\partial y}\left(\left(1+\nu_{t} / \sigma_{\kappa}\right) \frac{\partial \kappa}{\partial y}\right)\right|_{i, j, k} ^{n} & \approx \frac{1}{\delta y}\left[\left.\left(1+\frac{\nu_{t}}{\sigma_{\kappa}}\right)\left(\frac{\partial \kappa}{\partial y}\right)\right|_{i, j+\frac{1}{2}, k} ^{n}-\left.\left(1+\frac{\nu_{t}}{\sigma_{\kappa}}\right)\left(\frac{\partial \kappa}{\partial y}\right)\right|_{i, j-\frac{1}{2}, k} ^{n}\right] \\
& \approx \frac{1}{\delta y}\left[\left(1+\frac{\nu_{t_{i, j+\frac{1}{2}, k}}^{n}}{\sigma_{\kappa}}\right)\left(\frac{\kappa_{i, j+1, k}-\kappa_{i, j, k}}{\delta y}\right)\right. \\
& \left.\left(1+\frac{\nu_{t_{i, j-\frac{1}{2}, k}}^{n}}{\sigma_{\kappa}}\right)\left(\frac{\kappa_{i, j, k}-\kappa_{i, j, k-1}}{\delta y}\right)\right],
\end{aligned}
$$

onde

$$
\nu_{t_{i, j+\frac{1}{2}, k}}^{n} \approx 0.5\left(\nu_{t_{i, j+1, k}}^{n}+\nu_{t_{i, j, k}}^{n}\right), \quad \text { e } \quad \nu_{t_{i, j-\frac{1}{2}, k}}^{n} \approx 0.5\left(\nu_{t_{i, j, k}}^{n}+\nu_{t_{i, j-1, k}}^{n}\right) .
$$

Os termos convectivos definidos nas Eqs. (4.48)-(4.52) e (4.72)-(4.77) são aproximados pelo esquema TDPUS-C3. Na sequência são apresentadas apenas as discretizações de dois representantes típicos de tais termos não lineares, isto é, à do termo $C O N V(u)$ avaliado no ponto $\mathcal{P}=\left(i+\frac{1}{2}, j, k\right)$ e $C O N V(\kappa)$ avaliado no ponto $\mathcal{P}=(i, j, k)$. A discretização dos outros termos convectivos segue procedimentos similares. Para o termo $\left.C O N V(u)\right|_{i+\frac{1}{2}, j, k}$, tem-se:

$$
\begin{aligned}
& \left.C O N V(u)\right|_{i+\frac{1}{2}, j, k}=\left.\left(\frac{\partial(u u)}{\partial x}+\frac{\partial(u v)}{\partial y}+\frac{\partial(u w)}{\partial z}\right)\right|_{i+\frac{1}{2}, j, k}=\left.\frac{\partial(u u)}{\partial x}\right|_{i+\frac{1}{2}, j, k}+\left.\frac{\partial(u v)}{\partial y}\right|_{i+\frac{1}{2}, j, k}+\left.\frac{\partial(u w)}{\partial z}\right|_{i+\frac{1}{2}, j, k} \\
& \approx \frac{\bar{u}_{1} u_{i+1, j, k}-\bar{u}_{2} u_{i, j, k}}{\delta x}+\frac{\bar{v}_{1} u_{i+\frac{1}{2}, j+\frac{1}{2}, k}-\bar{v}_{2} u_{i+\frac{1}{2}, j-\frac{1}{2}, k}}{\delta y}+\frac{\bar{w}_{1} u_{i+\frac{1}{2}, j, k+\frac{1}{2}}-\bar{w}_{2} u_{i+\frac{1}{2}, j, k-\frac{1}{2}}}{\delta z}
\end{aligned}
$$

em que $\bar{u}_{1}, \bar{u}_{2}, \bar{v}_{1}, \bar{v}_{2}, \bar{w}_{1}$, e $\bar{w}_{2}$ são velocidades (médias) de advecção; por exemplo, $\bar{v}_{1}$ e $\bar{v}_{2}$ são obtidas como

$$
\bar{v}_{1}=\bar{v}_{i+\frac{1}{2}, j+\frac{1}{2}, k} \approx \frac{1}{2}\left(v_{i+1, j+\frac{1}{2}, k}+v_{i, j+\frac{1}{2}, k}\right), \quad \bar{v}_{2}=\bar{v}_{i+\frac{1}{2}, j-\frac{1}{2}, k} \approx \frac{1}{2}\left(v_{i+1, j-\frac{1}{2}, k}+v_{i, j-\frac{1}{2}, k}\right) .
$$

Na Eq. (4.106), a variável convectada $u$ nos pontos $(i+1, j, k),(i, j, k),\left(i+\frac{1}{2}, j+\frac{1}{2}, k\right),\left(i+\frac{1}{2}, j-\right.$ $\left.\frac{1}{2}, k\right),\left(i+\frac{1}{2}, j, k+\frac{1}{2}\right)$ e $\left(i+\frac{1}{2}, j, k-\frac{1}{2}\right)$ é calculada usando-se o novo esquema convectivo TDPUS-C3. Sem perda de generalidade apresentam-se a seguir, com o auxílio das posições à jusante $D$, à montante $U$ e mais à montante $R$, os cálculos de $u_{i+\frac{1}{2}, j+\frac{1}{2}, k}$ e $u_{i+\frac{1}{2}, j-\frac{1}{2}, k}$ (ou, equivalentemente, uma aproximação para $\frac{\partial(u v)}{\partial y}$ no ponto $\left.\mathcal{P}=\left(i+\frac{1}{2}, j, k\right)\right)$.

(1) Cálculo de $u_{i+\frac{1}{2}, j+\frac{1}{2}, k}$ em função da normalização $\hat{u}_{U}=\frac{u_{U}-u_{R}}{u_{D}-u_{R}}$ (ver Eq. (2.6)). 
Se $\bar{v}_{1}=v_{i+\frac{1}{2}, j+\frac{1}{2}, k}>0$, então

$$
u_{i+\frac{1}{2}, j+\frac{1}{2}, k}= \begin{cases}u_{i+\frac{1}{2}, j-1, k}+\left(u_{i+\frac{1}{2}, j+1, k}-u_{i+\frac{1}{2}, j-1, k}\right) *\left[\hat{u}_{U}+\hat{u}_{U}^{4}\left(\alpha_{4}+\hat{u}_{U}\left(\alpha_{5}\right.\right.\right. & \\ \left.\left.+\hat{u}_{U}\left(\alpha_{6}+\hat{u}_{U}\left(\alpha_{7}+\hat{u}_{U}\left(\alpha_{8}+\hat{u}_{U}\left(\alpha_{9}+\alpha_{10} \hat{u}_{U}\right)\right)\right)\right)\right)\right], & \hat{u}_{U} \in[0,1] ; \\ u_{i+\frac{1}{2}, j, k}, & \hat{u}_{U} \notin[0,1],\end{cases}
$$

onde

$$
D=\left(i+\frac{1}{2}, j+1, k\right), \quad U=\left(i+\frac{1}{2}, j, k\right), \quad R=\left(i+\frac{1}{2}, j-1, k\right)
$$

Se $\bar{v}_{1}=v_{i+\frac{1}{2}, j+\frac{1}{2}, k}<0$, então

$$
u_{i+\frac{1}{2}, j+\frac{1}{2}, k}= \begin{cases}u_{i+\frac{1}{2}, j+2, k}+\left(u_{i+\frac{1}{2}, j, k}-u_{i+\frac{1}{2}, j+2, k}\right) *\left[\hat{u}_{U}+\hat{u}_{U}^{4}\left(\alpha_{4}+\hat{u}_{U}\left(\alpha_{5}\right.\right.\right. & \\ \left.+\hat{u}_{U}\left(\alpha_{6}+\hat{u}_{U}\left(\alpha_{7}+\hat{u}_{U}\left(\alpha_{8}+\hat{u}_{U}\left(\alpha_{9}+\alpha_{10} \hat{u}_{U}\right)\right)\right)\right)\right], & \hat{u}_{U} \in[0,1] ; \\ u_{i+\frac{1}{2}, j+1, k}, & \hat{u}_{U} \notin[0,1],\end{cases}
$$

onde

$$
D=\left(i+\frac{1}{2}, j, k\right), \quad U=\left(i+\frac{1}{2}, j+1, k\right), \quad R=\left(i+\frac{1}{2}, j+2, k\right) .
$$

(2) $u_{i+\frac{1}{2}, j-\frac{1}{2}, k}$ em função da normalização $\hat{u}_{U}=\frac{u_{U}-u_{R}}{u_{D}-u_{R}}$.

Se $\bar{v}_{2}=v_{i+\frac{1}{2}, j-\frac{1}{2}, k}>0$, então

$$
u_{i+\frac{1}{2}, j-\frac{1}{2}, k}= \begin{cases}u_{i+\frac{1}{2}, j-2, k}+\left(u_{i+\frac{1}{2}, j, k}-u_{i+\frac{1}{2}, j-2, k}\right) *\left[\hat{u}_{U}+\hat{u}_{U}^{4}\left(\alpha_{4}+\hat{u}_{U}\left(\alpha_{5}\right.\right.\right. & \\ \left.\left.+\hat{u}_{U}\left(\alpha_{6}+\hat{u}_{U}\left(\alpha_{7}+\hat{u}_{U}\left(\alpha_{8}+\hat{u}_{U}\left(\alpha_{9}+\alpha_{10} \hat{u}_{U}\right)\right)\right)\right)\right)\right], & \hat{u}_{U} \in[0,1] ; \\ u_{i+\frac{1}{2}, j-1, k}, & \hat{u}_{U} \notin[0,1],\end{cases}
$$

onde

$$
D=\left(i+\frac{1}{2}, j, k\right), \quad U=\left(i+\frac{1}{2}, j-1, k\right), \quad R=\left(i+\frac{1}{2}, j-2, k\right)
$$

Se $\bar{v}_{2}=v_{i+\frac{1}{2}, j-\frac{1}{2}, k}<0$, então

$$
u_{i+\frac{1}{2}, j-\frac{1}{2}, k}= \begin{cases}u_{i+\frac{1}{2}, j+1, k}+\left(u_{i+\frac{1}{2}, j-1, k}-u_{i+\frac{1}{2}, j+1, k}\right) *\left[\hat{u}_{U}+\hat{u}_{U}^{4}\left(\alpha_{4}+\hat{u}_{U}\left(\alpha_{5}\right.\right.\right. & \\ \left.\left.+\hat{u}_{U}\left(\alpha_{6}+\hat{u}_{U}\left(\alpha_{7}+\hat{u}_{U}\left(\alpha_{8}+\hat{u}_{U}\left(\alpha_{9}+\alpha_{10} \hat{u}_{U}\right)\right)\right)\right)\right)\right], & \hat{u}_{U} \in[0,1] ; \\ u_{i+\frac{1}{2}, j, k}, & \hat{u}_{U} \notin[0,1],\end{cases}
$$

onde

$$
D=\left(i+\frac{1}{2}, j-1, k\right), \quad U=\left(i+\frac{1}{2}, j, k\right), \quad R=\left(i+\frac{1}{2}, j+1, k\right) .
$$

Os coeficientes $\alpha_{i}, i=4, \ldots, 10$, que aparecem anteriormente estão definidos em (2.56). 
Para o termo $\left.C O N V(\kappa)\right|_{i, j, k}$, tem-se:

$$
\begin{gathered}
\left.\operatorname{CONV}(\kappa)\right|_{i, j, k}=\left.\left(\frac{\partial(\kappa u)}{\partial x}+\frac{\partial(\kappa v)}{\partial y}+\frac{\partial(\kappa w)}{\partial z}\right)\right|_{i, j, k} \approx \\
\frac{\bar{u}_{1} \kappa_{i+\frac{1}{2}, j, k}-\bar{u}_{2} \kappa_{i-\frac{1}{2}, j, k}}{\delta x}+\frac{\bar{v}_{1} \kappa_{i, j+\frac{1}{2}, k}-\bar{v}_{2} \kappa_{i, j-\frac{1}{2}, k}}{\delta y}+\frac{\bar{w}_{1} \kappa_{i, j, k+\frac{1}{2}}-\bar{w}_{2} \kappa_{i, j, k-\frac{1}{2}}}{\delta z}
\end{gathered}
$$

em que $\bar{u}_{1}, \bar{u}_{2}, \bar{v}_{1}, \bar{v}_{2}, \bar{w}_{1}$, e $\bar{w}_{2}$ são obtidas por

$$
\bar{u}_{1}=u_{i+\frac{1}{2}, j, k}, \bar{u}_{2}=u_{i-\frac{1}{2}, j, k}, \bar{v}_{1}=u_{i, j+\frac{1}{2}, k}, \bar{v}_{2}=u_{i, j-\frac{1}{2}, k}, \bar{w}_{1}=u_{i, j, k+\frac{1}{2}} \text { e } \quad \bar{w}_{2}=u_{i, j, k-\frac{1}{2}} .
$$

Da mesma forma como foi feito para o cálculo da variável convectada $u$, a variável convectada $\kappa$ na Eq. (4.108), nos pontos $\left(i+\frac{1}{2}, j, k\right),\left(i-\frac{1}{2}, j, k\right),\left(i, j+\frac{1}{2}, k\right),\left(i, j-\frac{1}{2}, k\right),\left(i, j, k+\frac{1}{2}\right),\left(i, j, k-\frac{1}{2}\right)$, é calculada usando-se o novo esquema convectivo TDPUS-C3. Novamente, sem perda de generalidade, apresentam-se a seguir com o auxílio dos posições $D, U$ e $R$ os cálculos de $\kappa_{i+\frac{1}{2}, j, k}, \kappa_{i-\frac{1}{2}, j, k}, \kappa_{i, j+\frac{1}{2}, k}$, $\kappa_{i, j-\frac{1}{2}, k}, \kappa_{i, j, k+\frac{1}{2}}, \kappa_{i, j, k-\frac{1}{2}}$ (ou, equivalentemente, uma aproximação para $\frac{\partial(\kappa v)}{\partial y}$ em $(i, j, k)$ ).

(1) Cálculo de $\kappa_{i, j+\frac{1}{2}, k}$ em função da normalização $\hat{\kappa}_{U}=\frac{\kappa_{U}-\kappa_{R}}{\kappa_{D}-\kappa_{R}}$.

Se $\bar{v}_{1}=v_{i, j+\frac{1}{2}, k}>0$ então

$$
\kappa_{i, j+\frac{1}{2}, k}= \begin{cases}\kappa_{i, j-1, k}+\left(\kappa_{i, j+1, k}-\kappa_{i, j-1, k}\right) *\left[\hat{\kappa}_{U}+\hat{\kappa}_{U}^{4}\left(\alpha_{4}+\hat{\kappa}_{U}\left(\alpha_{5}\right.\right.\right. & \\ \left.\left.+\hat{\kappa}_{U}\left(\alpha_{6}+\hat{\kappa}_{U}\left(\alpha_{7}+\hat{\kappa}_{U}\left(\alpha_{8}+\hat{\kappa}_{U}\left(\alpha_{9}+\alpha_{10} \hat{\kappa}_{U}\right)\right)\right)\right)\right)\right], & \hat{\kappa}_{U} \in[0,1] ; \\ \kappa_{i, j, k}, & \hat{\kappa}_{U} \notin[0,1],\end{cases}
$$

onde

$$
D=(i, j+1, k), \quad U=(i, j, k), \quad R=(i, j-1, k) ;
$$

Se $\bar{v}_{1}=v_{i, j+\frac{1}{2}, k}<0$, então

$$
\kappa_{i, j+\frac{1}{2}, k}= \begin{cases}\kappa_{i, j+2, k}+\left(\kappa_{i, j, k}-\kappa_{i, j+2, k}\right) *\left[\hat{\kappa}_{U}+\hat{\kappa}_{U}^{4}\left(\alpha_{4}+\hat{\kappa}_{U}\left(\alpha_{5}\right.\right.\right. & \\ \left.\left.+\hat{\kappa}_{U}\left(\alpha_{6}+\hat{\kappa}_{U}\left(\alpha_{7}+\hat{\kappa}_{U}\left(\alpha_{8}+\hat{\kappa}_{U}\left(\alpha_{9}+\alpha_{10} \hat{\kappa}_{U}\right)\right)\right)\right)\right)\right], & \hat{\kappa}_{U} \in[0,1] ; \\ \kappa_{i, j+1, k}, & \hat{\kappa}_{U} \notin[0,1],\end{cases}
$$

onde

$$
D=(i, j, k), \quad U=(i, j+1, k), \quad R=(i, j+2, k) .
$$

(2) $\kappa_{i, j-\frac{1}{2}, k}$, em função da normalização $\hat{\kappa}_{U}=\frac{\kappa_{U}-\kappa_{R}}{\kappa_{D}-\kappa_{R}}$.

Se $\bar{v}_{2}=v_{i, j-\frac{1}{2}, k}>0$, então

$$
\kappa_{i, j-\frac{1}{2}, j, k}= \begin{cases}\kappa_{i, j-2, k}+\left(\kappa_{i, j, k}-\kappa_{i, j-2, k}\right) *\left[\hat{\kappa}_{U}+\hat{\kappa}_{U}^{4}\left(\alpha_{4}+\hat{\kappa}_{U}\left(\alpha_{5}\right.\right.\right. & \\ \left.\left.+\hat{\kappa}_{U}\left(\alpha_{6}+\hat{\kappa}_{U}\left(\alpha_{7}+\hat{\kappa}_{U}\left(\alpha_{8}+\hat{\kappa}_{U}\left(\alpha_{9}+\alpha_{10} \hat{\kappa}_{U}\right)\right)\right)\right)\right)\right], & \hat{\kappa}_{U} \in[0,1] ; \\ \kappa_{i, j-1, k}, & \hat{\kappa}_{U} \notin[0,1],\end{cases}
$$


onde

$$
D=(i, j, k), \quad U=(i, j-1, k), \quad R=(i, j-2, k)
$$

Se $\bar{v}_{2}=v_{i, j-\frac{1}{2}, k}<0$, então

$$
\kappa_{i, j-\frac{1}{2}, k}= \begin{cases}\kappa_{i, j+1, k}+\left(\kappa_{i, j-1, k}-\kappa_{i, j+1, k}\right) *\left[\hat{\kappa}_{U}+\hat{\kappa}_{U}^{4}\left(\alpha_{4}+\hat{\kappa}_{U}\left(\alpha_{5}\right.\right.\right. & \\ \left.\left.+\hat{\kappa}_{U}\left(\alpha_{6}+\hat{\kappa}_{U}\left(\alpha_{7}+\hat{\kappa}_{U}\left(\alpha_{8}+\hat{\kappa}_{U}\left(\alpha_{9}+\alpha_{10} \hat{\kappa}_{U}\right)\right)\right)\right)\right)\right], & \hat{\kappa}_{U} \in[0,1] ; \\ \kappa_{i, j, k}, & \hat{\kappa}_{U} \notin[0,1],\end{cases}
$$

onde

$$
D=(i, j-1, k), \quad U=(i, j, k), \quad R=(i, j+1, k) .
$$

As constantes $\alpha_{i=4, \ldots, 10}$ estão definidas previamente.

Em resumo, as equações de trabalho discretas para simular escoamentos incompressíveis de fluidos newtonianos com a "turbulência ligada" ( $\alpha=1)$ são como segue.

- Equação do movimento na direção $x$ :

$$
\begin{aligned}
& \tilde{u}_{i+\frac{1}{2}, j, k}^{n+1}=u_{i+\frac{1}{2}, j, k}^{n}+\delta t\left\{\left.C O N V(u)\right|_{i+\frac{1}{2}, j, k}-\frac{\tilde{p}_{e_{i+1, j, k}}-\tilde{p}_{e_{i, j, k}}}{\delta x}\right. \\
& +\frac{1}{\operatorname{Re} \delta y}\left[\left(\frac{u_{i+\frac{1}{2}, j+1, k}-u_{i+\frac{1}{2}, j, k}}{\delta y}-\frac{v_{i+1, j+\frac{1}{2}, k}-v_{i, j+\frac{1}{2}, k}}{\delta x}\right)\right. \\
& \left.-\left(\frac{u_{i+\frac{1}{2}, j, k}-u_{i+\frac{1}{2}, j-1, k}}{\delta y}\right)-\left(\frac{v_{i+1, j-\frac{1}{2}, k}-v_{i, j-\frac{1}{2}, k}}{\delta x}\right)\right] \\
& +\frac{1}{\operatorname{Re} \delta z}\left[\left(\frac{u_{i+\frac{1}{2}, j, k+1}-u_{i+\frac{1}{2}, j, k}}{\delta y}-\frac{w_{i+1, j, k+\frac{1}{2}}-w_{i, j, k+\frac{1}{2}}}{\delta x}\right)\right. \\
& \left.-\left(\frac{u_{i+\frac{1}{2}, j, k}-u_{i+\frac{1}{2}, j, k-1}}{\delta z}\right)-\left(\frac{w_{i+1, j, k-\frac{1}{2}, k}-w_{i, j, k-\frac{1}{2}}}{\delta x}\right)\right] \\
& +\alpha\left(\frac{2}{\operatorname{Re} \delta x^{2}}\left[\nu_{t_{i+1, j, k}}\left(u_{i+\frac{3}{2}, j, k}-u_{i+\frac{1}{2}, j, k}\right)-\nu_{t_{i+1, j, k}}\left(u_{i+\frac{3}{2}, j, k}-u_{i+\frac{1}{2}, j, k}\right)\right]\right. \\
& +\frac{1}{R e \delta y}\left[\nu_{t_{i+\frac{1}{2}, j+\frac{1}{2}, k}}\left(\frac{u_{i+\frac{1}{2}, j+1, k}-u_{i+\frac{1}{2}, j, k}}{\delta y}+\frac{v_{i+1, j+\frac{1}{2}, k}-v_{i, j+\frac{1}{2}, k}}{\delta x}\right)\right. \\
& \left.-\nu_{t_{i+\frac{1}{2}, j-\frac{1}{2}, k}}\left(\frac{u_{i+\frac{1}{2}, j, k}-u_{i+\frac{1}{2}, j-1, k}}{\delta y}+\frac{v_{i+1, j-\frac{1}{2}, k}-v_{i, j-\frac{1}{2}, k}}{\delta x}\right)\right] \\
& +\frac{1}{\operatorname{Re} \delta z}\left[\nu_{t_{i+\frac{1}{2}, j, k+\frac{1}{2}}}\left(\frac{u_{i+\frac{1}{2}, j, k+1}-u_{i+\frac{1}{2}, j, k}}{\delta y}+\frac{w_{i+1, j, k+\frac{1}{2}}-w_{i, j+\frac{1}{2}, k+\frac{1}{2}}}{\delta x}\right)\right. \\
& \left.\left.\left.-\nu_{t_{i+\frac{1}{2}, j, k-\frac{1}{2}}}\left(\frac{u_{i+\frac{1}{2}, j, k}-u_{i+\frac{1}{2}, j, k-1}}{\delta z}+\frac{w_{i+1, j, k-\frac{1}{2}}-w_{i, j, k-\frac{1}{2}}}{\delta x}\right)\right]\right)+\frac{1}{F r^{2}} g_{x}\right\}^{n} .
\end{aligned}
$$


- Equação do movimento na direção $y$ :

$$
\begin{aligned}
& \tilde{v}_{i, j+\frac{1}{2}, k}^{n+1}=v_{i, j+\frac{1}{2}, k}^{n}+\delta t\left\{\left.C O N V(v)\right|_{i, j+\frac{1}{2}, k}-\frac{\tilde{p}_{e_{i, j+1, k}}-\tilde{p}_{e_{i, j, k}}}{\delta y}\right. \\
& +\frac{1}{\operatorname{Re} \delta x}\left[\left(\frac{u_{i+\frac{1}{2}, j+1, k}-u_{i+\frac{1}{2}, j, k}}{\delta y}-\frac{v_{i+1, j+\frac{1}{2}, k}-v_{i, j+\frac{1}{2}, k}}{\delta x}\right)\right. \\
& \left.-\left(\frac{u_{i-\frac{1}{2}, j+1, k}-u_{i-\frac{1}{2}, j, k}}{\delta y}\right)-\left(\frac{v_{i, j+\frac{1}{2}, k}-v_{i-1, j+\frac{1}{2}, k}}{\delta x}\right)\right] \\
& +\frac{1}{\operatorname{Re} \delta z}\left[\left(\frac{v_{i, j+\frac{1}{2}, k+1}-v_{i, j+\frac{1}{2}, k}}{\delta y}-\frac{w_{i, j+1, k+\frac{1}{2}}-w_{i, j, k+\frac{1}{2}}}{\delta y}\right)\right. \\
& \left.-\left(\frac{v_{i, j+\frac{1}{2}, k}-v_{i, j+\frac{1}{2}, k-1}}{\delta z}\right)-\left(\frac{w_{i, j+1, k-\frac{1}{2}}-w_{i, j, k-\frac{1}{2}}}{\delta y}\right)\right] \\
& +\alpha\left(\frac{2}{\operatorname{Re} \delta y^{2}}\left[\nu_{t_{i, j+1, k}}\left(v_{i, j+\frac{3}{2}, k}-v_{i, j+\frac{1}{2}, k}\right)-\nu_{t_{i, j, k}}\left(v_{i, j+\frac{1}{2}, k}-v_{i, j-\frac{1}{2}, k}\right)\right]\right. \\
& +\frac{1}{R e \delta x}\left[\nu_{t_{i+\frac{1}{2}, j+\frac{1}{2}, k}}\left(\frac{u_{i+\frac{1}{2}, j+1, k}-u_{i+\frac{1}{2}, j, k}}{\delta y}+\frac{v_{i+1, j+\frac{1}{2}, k}-v_{i, j+\frac{1}{2}, k}}{\delta x}\right)\right. \\
& \left.-\nu_{t_{i-\frac{1}{2}, j+\frac{1}{2}, k}}\left(\frac{u_{i+\frac{1}{2}, j+1, k}-u_{i-\frac{1}{2}, j, k}}{\delta y}+\frac{v_{i, j+\frac{1}{2}, k}-v_{i-1, j+\frac{1}{2}, k}}{\delta x}\right)\right] \\
& +\frac{1}{\operatorname{Re} \delta z}\left[\nu_{t_{i, j+\frac{1}{2}, k+\frac{1}{2}}}\left(\frac{v_{i, j+\frac{1}{2}, k+1}-v_{i, j+\frac{1}{2}, k}}{\delta z}+\frac{w_{i, j+1, k+\frac{1}{2}}-w_{i, j, k+\frac{1}{2}}}{\delta y}\right)\right. \\
& \left.\left.\left.-\nu_{t_{i, j+\frac{1}{2}, k-\frac{1}{2}}}\left(\frac{v_{i, j+\frac{1}{2}, k}-v_{i, j+\frac{1}{2}, k-1}}{\delta z}+\frac{w_{i, j+1, k-\frac{1}{2}}-w_{i, j, k-\frac{1}{2}}}{\delta y}\right)\right]\right)+\frac{1}{F r^{2}} g_{y}\right\}^{n} .
\end{aligned}
$$

- Equação do movimento na direção $z$ :

$$
\begin{aligned}
\tilde{w}_{i, j, k+\frac{1}{2}}^{n+1}= & w_{i, j, k+\frac{1}{2}}^{n}+\delta t\left\{\left.\operatorname{CONV}(w)\right|_{i, j, k+\frac{1}{2}}-\frac{\tilde{p}_{e_{i, j+1, k}}-\tilde{p}_{e_{i, j, k}}}{\delta z}\right. \\
& +\frac{1}{\operatorname{Re} \delta x}\left[\left(\frac{u_{i+\frac{1}{2}, j, k+1}-u_{i+\frac{1}{2}, j, k}}{\delta z}-\frac{w_{i+1, j, k+\frac{1}{2}}-w_{i, j, k+\frac{1}{2}}}{\delta x}\right)\right. \\
& \left.-\left(\frac{u_{i-\frac{1}{2}, j, k+1}-u_{i-\frac{1}{2}, j, k}}{\delta z}\right)-\left(\frac{w_{i, j, k+\frac{1}{2}}-w_{i-1, j, k+\frac{1}{2}}}{\delta x}\right)\right] \\
& +\frac{1}{\operatorname{Re} \delta y}\left[\left(\frac{v_{i, j+\frac{1}{2}, k+1}-v_{i, j+\frac{1}{2}, k}}{\delta z}-\frac{w_{i, j+1, k+\frac{1}{2}}-w_{i, j, k+\frac{1}{2}}}{\delta y}\right)\right. \\
& -\left(\frac{v_{i, j-\frac{1}{2}, k+1}-v_{i, j-\frac{1}{2}, k}}{\delta z}-\left(\frac{w_{i, j, k+\frac{1}{2}}-w_{i, j-1, k+\frac{1}{2}}}{\delta y}\right)\right] \\
& +\alpha\left(\frac{2}{R e \delta z^{2}}\left[\nu_{t_{i, j, k+1}}\left(w_{i, j, k+\frac{3}{2}}-w_{i, j, k+\frac{1}{2}}\right)-\nu_{t_{i, j, k}}\left(w_{i, j, k+\frac{1}{2}}-w_{i, j, k-\frac{1}{2}}\right)\right]\right. \\
+ & \frac{1}{\operatorname{Re} \delta x}\left[\nu_{t_{i+\frac{1}{2}, j, k+\frac{1}{2}}}\left(\frac{\left.u_{i+\frac{1}{2}, j, k+1}-u_{i+\frac{1}{2}, j, k}+\frac{w_{i+1, j, k+\frac{1}{2}}-w_{i, j, k+\frac{1}{2}}}{\delta z}\right)}{\delta x}\right)\right. \\
& \left.-\nu_{t_{i-\frac{1}{2}, j, k+\frac{1}{2}}}\left(\frac{u_{i-\frac{1}{2}, j, k+1}-u_{i-\frac{1}{2}, j, k}}{\delta z}+\frac{w_{i, j, k+\frac{1}{2}}-w_{i-1, j, k+\frac{1}{2}}}{\delta x}\right)\right]
\end{aligned}
$$




$$
\begin{aligned}
& +\frac{1}{\operatorname{Re} \delta y}\left[\nu_{t_{i, j+\frac{1}{2}, k+\frac{1}{2}}}\left(\frac{v_{i, j+\frac{1}{2}, k+1}-v_{i, j+\frac{1}{2}, k}}{\delta z}+\frac{w_{i, j+1, k+\frac{1}{2}}-w_{i, j, k+\frac{1}{2}}}{\delta y}\right)\right. \\
& \left.\left.\left.-\nu_{t_{i, j-\frac{1}{2}, k+\frac{1}{2}}}\left(\frac{v_{i, j+\frac{1}{2}, k+1}-v_{i, j+\frac{1}{2}, k}}{\delta z}+\frac{w_{i, j, k+\frac{1}{2}}-w_{i, j-1, k+\frac{1}{2}}}{\delta y}\right)\right]\right)+\frac{1}{F r^{2}} g_{z}\right\}^{n} .
\end{aligned}
$$

- Equação de Poisson:

$$
\begin{gathered}
\frac{\psi_{i+1, j, k}-2 \psi_{i, j, k}+\psi_{i-1, j, k}}{\delta x^{2}}+\frac{\psi_{i, j+1, k}-2 \psi_{i, j, k}+\psi_{i, j-1, k}}{\delta y^{2}}+\frac{\psi_{i, j, k+1}-2 \psi_{i, j, k}+\psi_{i, j, k-1}}{\delta z^{2}} \\
=\frac{\tilde{u}_{i+\frac{1}{2}, j, k}-\tilde{u}_{i-\frac{1}{2}, j, k}}{\delta x}+\frac{\tilde{v}_{i+\frac{1}{2}, j, k}-\tilde{v}_{i-\frac{1}{2}, j, k}}{\delta y}+\frac{\tilde{w}_{i, j, k+\frac{1}{2}}-\tilde{w}_{i, j, k-\frac{1}{2}}}{\delta x} .
\end{gathered}
$$

- Energia cinética $\kappa$ :

$$
\begin{array}{r}
\kappa_{i, j, k}^{n+1}=\kappa_{i, j, k}^{n}+\delta t\left\{\left.\operatorname{CONV}(\kappa)\right|_{i, j, k}+\frac{1}{R e}\left[\frac { 1 } { \delta x ^ { 2 } } \left(\left(1+\frac{\nu_{t_{i+1, j, k}}-\nu_{t_{i, j, k}}}{2 \sigma_{\kappa}}\right)\left(\kappa_{i+1, j, k}-\kappa_{i, j, k}\right)\right.\right.\right. \\
\left.-\left(1+\frac{\nu_{t_{i, j, k}}-\nu_{t_{i-1, j, k}}}{2 \sigma_{\kappa}}\right)\left(\kappa_{i, j, k}-\kappa_{i-1, j, k}\right)\right)+\frac{1}{\delta y^{2}}\left(\left(1+\frac{\nu_{t_{i, j+1, k}}-\nu_{t_{i, j, k}}}{2 \sigma_{\kappa}}\right)\left(\kappa_{i, j+1, k}-\kappa_{i, j, k}\right)\right. \\
\left.-\left(1+\frac{\nu_{t_{i, j, k}}-\nu_{t_{i, j-1, k}}}{2 \sigma_{\kappa}}\right)\left(\kappa_{i, j, k}-\kappa_{i, j-1, k}\right)\right)+\frac{1}{\delta z^{2}}\left(\left(1+\frac{\nu_{t_{i, j, k+1}}-\nu_{t_{i, j, k}}}{2 \sigma_{\kappa}}\right)\left(\kappa_{i, j, k+1}-\kappa_{i, j, k}\right)\right. \\
\left.\left.\left.-\left(1+\frac{\nu_{t_{i, j, k}}-\nu_{t_{i, j-1, k}}}{2 \sigma_{\kappa}}\right)\left(\kappa_{i, j, k}-\kappa_{i, j-1, k}\right)\right)\right]+P_{i, j, k}-\varepsilon_{i, j, k}\right\}^{n} .
\end{array}
$$

- Dissipação da energia $\varepsilon$ :

$$
\begin{gathered}
\varepsilon_{i, j, k}^{n+1}=\varepsilon_{i, j, k}^{n}+\delta t\left\{\left.\operatorname{CONV}(\varepsilon)\right|_{i, j, k}+\frac{1}{R e}\left[\frac { 1 } { \delta x ^ { 2 } } \left(\left(1+\frac{\nu_{t_{i+1, j, k}}-\nu_{t_{i, j, k}}}{2 \sigma_{\varepsilon}}\right)\left(\varepsilon_{i+1, j, k}-\varepsilon_{i, j, k}\right)\right.\right.\right. \\
\left.-\left(1+\frac{\nu_{t_{i, j, k}}-\nu_{t_{i-1, j, k}}}{2 \sigma_{\varepsilon}}\right)\left(\varepsilon_{i, j, k}-\varepsilon_{i-1, j, k}\right)\right)+\frac{1}{\delta y^{2}}\left(\left(1+\frac{\nu_{t_{i, j+1, k}}-\nu_{t_{i, j, k}}}{2 \sigma_{\varepsilon}}\right)\left(\varepsilon_{i, j+1, k}-\varepsilon_{i, j, k}\right)\right. \\
\left.-\left(1+\frac{\nu_{t_{i, j, k}}-\nu_{t_{i, j-1, k}}}{2 \sigma_{\varepsilon}}\right)\left(\varepsilon_{i, j, k}-\varepsilon_{i, j-1, k}\right)\right)+\frac{1}{\delta z^{2}}\left(\left(1+\frac{\nu_{t_{i, j, k+1}}-\nu_{t_{i, j, k}}}{2 \sigma_{\varepsilon}}\right)\left(\varepsilon_{i, j, k+1}-\varepsilon_{i, j, k}\right)\right. \\
\left.\left.\left.-\left(1+\frac{\nu_{t_{i, j, k}}-\nu_{t_{i, j-1, k}}}{2 \sigma_{\varepsilon}}\right)\left(\varepsilon_{i, j, k}-\varepsilon_{i, j, k-1}\right)\right)\right]+\left(C_{1 \varepsilon} P_{i, j, k}-C_{2 \varepsilon} \varepsilon_{i, j, k} / T_{i, j, k}\right)\right\}^{n} .
\end{gathered}
$$


- Produção de turbulência:

$$
\begin{aligned}
P_{i, j, k}^{n}=\nu_{t_{i, j, k}}^{n}\{ & \left\{\left[\frac{2}{\delta x^{2}}\left(u_{i+\frac{1}{2}, j, k}-u_{i-\frac{1}{2}, j-1, k}\right)^{2}+\frac{2}{\delta y^{2}}\left(v_{i, j+\frac{1}{2}, k}-v_{i, j-\frac{1}{2}, k}\right)^{2}+\frac{2}{\delta z^{2}}\left(w_{i, j, k+\frac{1}{2}}-w_{i, j, k-\frac{1}{2}}\right)^{2}\right]\right. \\
& +\left[\frac{1}{4 \delta y}\left(u_{i+\frac{1}{2}, j+1, k}+u_{i-\frac{1}{2}, j+1, k}-u_{i+\frac{1}{2}, j-1, k}-u_{i-\frac{1}{2}, j-1, k}\right)\right. \\
& \left.+\frac{1}{4 \delta x}\left(v_{i+1, j+\frac{1}{2}, k}+v_{i+1, j-\frac{1}{2}, k}-v_{i-1, j+\frac{1}{2}, k}-v_{i-1, j-\frac{1}{2}, k}\right)\right]^{2} \\
& +\left[\frac{1}{4 \delta z}\left(u_{i+\frac{1}{2}, j, k+1}+u_{i-\frac{1}{2}, j, k+1}-u_{i+\frac{1}{2}, j, k-1}-u_{i-\frac{1}{2}, j, k-1}\right)\right. \\
& \left.+\frac{1}{4 \delta x}\left(w_{i+1, j, k+\frac{1}{2}}+w_{i+1, j, k-\frac{1}{2}}-w_{i-1, j, k+\frac{1}{2}}-w_{i-1, j, k-\frac{1}{2}}\right)\right]^{2} \\
& +\left[\frac{1}{4 \delta z}\left(v_{i, j+\frac{1}{2}, k+1}+v_{i, j-\frac{1}{2}, k+1}-v_{i, j+\frac{1}{2}, j, k-1}-v_{i, j-\frac{1}{2}, k-1}\right)\right. \\
& \left.\left.+\frac{1}{4 \delta y}\left(w_{i, j+1, k+\frac{1}{2}}+w_{i, j+1, j, k-\frac{1}{2}}-w_{i, j-1, k+\frac{1}{2}}-w_{i, j-1, k-\frac{1}{2}}\right)\right]^{2}\right\}^{n}
\end{aligned}
$$

- Escala de tempo turbulento e viscosidade turbulenta:

$$
T_{t_{i, j, k}}^{n}=\frac{\kappa_{i, j, k}^{n}}{\varepsilon_{i, j, k}^{n}}
$$

e

$$
\nu_{t_{i, j, k}}^{n}=C_{\mu} \kappa_{i, j, k} T_{t_{i, j, k}}^{n} .
$$

As equações de trabalho discretizadas para simular escoamentos incompressíveis em regime laminar são obtidas fazendo-se $\alpha=0$ nas Eqs. (4.110)-(4.112) e desprezando-se as equações correspondentes às variáveis turbulentas. As equações discretas para a simulação computacional de escoamentos viscoelásticos são obtidas de maneira semelhante às equações discretas para o caso newtoniano, cujas expressões foram omitidas aqui para economizar espaço; essas expressões completas podem ser encontradas em Figueiredo (2011).

\subsection{Condições adicionais adotadas e suas discretizações}

Nesta seção são apresentadas as condições adicionais (iniciais e de contorno) adotadas neste trabalho. Também, quando necessário, as discretizações dessas condições de contorno são apresentadas.

\subsubsection{Condições iniciais}

No geral, as variáveis dependentes são impostas no início dos cálculos. No caso de escoamentos em regime laminar, as variáveis u e $p$ são prescritas (em geral como nulas) em todo o domínio de cálculo. Em escoamentos em regime turbulento, as variáveis médias $\mathbf{u}$ e $p_{e}$ são também prescritas, como feito no caso laminar; para as variáveis $\kappa$ e $\varepsilon$, assumem-se as relações $\kappa=\kappa(I, U)=I U^{2}$, sendo $I$ uma intensidade de turbulência (tomada como 0.080 neste trabalho) e $U$ uma escala de velocidade (associada ao movimento das grandes escalas), e $\varepsilon=\varepsilon\left(\kappa, l_{t}\right)=\frac{\kappa^{3 / 2}}{l_{t}}$, sendo $l_{t}=\varsigma L$ (Elkaim et al., 1992); $L$ é uma 
escala de comprimento associada as grandes escalas e $\varsigma$ uma constante tomada neste trabalho como $3 \times 10^{-2}$; essas variáveis na forma adimensional são $\kappa=I R e$ e $\varepsilon=\frac{1}{\varsigma}\left(\frac{\kappa^{3}}{R e}\right)^{1 / 2}$, respectivamente. As condições iniciais adotadas para simular escoamentos viscoelásticos são prescrever as variáveis $\mathbf{u}, p$ e $\tau_{p}$ como nulas.

\subsubsection{Condições de contorno}

\subsubsection{Na entrada e na saída de fluido}

As condições de contorno na entrada do domínio de solução adotadas neste trabalho (tanto em problemas em regime laminar/turbulento quanto viscoelásticos) são de Dirichlet. Em particular, a velocidade de entrada pode assumir perfil reto ou parabólico. No caso de escoamentos turbulentos, as variáveis $\kappa$ e $\varepsilon$ são prescritas consistentemente com as condições iniciais impostas para essas variáveis. Em escoamentos viscoelásticos, a contribuição não newtoniana é prescrita de acordo com o perfil de velocidade adotado: $\boldsymbol{\tau}_{p}$ é nulo se o perfil de velocidades é reto; e no caso do perfil ser parabólico na direção $z$ adotam-se as relações (mais detalhes podem ser encontrados em (Oishi et al., 2011))

$$
\begin{aligned}
& \tau_{p_{x x}}=\tau_{p_{y y}}=\tau_{p_{x y}}=0, \\
& \tau_{p_{x z}}=\frac{1}{R e}\left(1-\frac{\lambda_{2}}{\lambda_{1}}\right) \frac{\partial w}{\partial x}, \\
& \tau_{p_{y z}}=\frac{1}{R e}\left(1-\frac{\lambda_{2}}{\lambda_{1}}\right) \frac{\partial w}{\partial y}, \\
& \tau_{p_{z z}}=2 \frac{W e}{R e}\left(1-\frac{\lambda_{2}}{\lambda_{1}}\right)\left[\left(\frac{\partial w}{\partial x}\right)^{2}+\left(\frac{\partial w}{\partial y}\right)^{2}\right] \text {. }
\end{aligned}
$$

As derivadas de primeira ordem na Eq. (4.119) são aproximadas por diferenças centrais.

Na região de saída de fluido, impõe-se como condição de contorno a condição homogênea de Neumann para todas as variáveis. A discretização dessa condição é feita usando diferença central, diferença avançada ou atrasada de primeira ordem.

\subsubsection{Na superfície livre do fluido}

Em qualquer tipo de escoamento estudado neste trabalho (laminar, turbulento ou viscoelástico), as condições de contorno para os componentes de velocidade $\left(u, v\right.$ e $w$ ) e pressão $\left(p\right.$ e $\left.p_{e}\right)$ adotadas na superfície livre do fluido são (na ausência dos efeitos de tensão superficial) dadas por (Landau e Lifschitz, 1980)

$$
\mathbf{n} \cdot(\boldsymbol{\sigma} \cdot \mathbf{n})=p_{\text {ext }}, \quad \mathbf{m} \mathbf{1} \cdot(\boldsymbol{\sigma} \cdot \mathbf{n})=0 \quad \text { e } \quad \mathbf{m} \mathbf{2} \cdot(\boldsymbol{\sigma} \cdot \mathbf{n})=0,
$$

em que $\mathbf{m} \mathbf{1}=\left(m 1_{x}, m 1_{y}, m 1_{z}\right)$ e $\mathbf{m} \mathbf{2}=\left(m 2_{x}, m 2_{y}, m 2_{z}\right)$ são vetores tangenciais unitários e externos à superfície livre, e $\mathbf{n}$ é um vetor unitário normal externo à ela. $p_{\text {ext }}$ é uma pressão externa (atmosférica) assumida nula neste trabalho. O tensor $\boldsymbol{\sigma}$ é dado por (4.3) para escoamentos (newtonianos ) laminares, dado por $\boldsymbol{\sigma}=(-p \mathbb{I}+\nu \mathbf{D})+\left(-p_{t} \mathbb{I}+\nu_{t} \mathbf{D}\right)$ para o regime turbulento, e dado pela Eq. (4.5) para escoamentos de fluidos viscoelásticos. As equações para as condições de contorno na superfície livre são obtidas substituindo-se a expressão do tensor $\boldsymbol{\sigma}$ ("laminar", "turbulento" ou "viscoelástico") nas Eqs. (4.120). Na forma adimensional, as equações correspondentes para o caso do regime turbulento são dadas por (obtêm-se as equações para o regime laminar ao se fazer $\nu_{t}=0$ ) 


$$
\begin{gathered}
p_{e}-\frac{2}{R e}\left(1+\nu_{t}\right)\left[\frac{\partial u}{\partial x} n_{x}^{2}+\frac{\partial v}{\partial y} n_{y}^{2}+\frac{\partial w}{\partial z} n_{z}^{2}+\left(\frac{\partial u}{\partial y}+\frac{\partial v}{\partial x}\right) n_{x} n_{y}+\right. \\
\left.\left(\frac{\partial u}{\partial z}+\frac{\partial w}{\partial x}\right) n_{x} n_{z}+\left(\frac{\partial v}{\partial z}+\frac{\partial w}{\partial y}\right) n_{y} n_{z}\right]=0 \\
\frac{1}{R e}\left(1+\nu_{t}\right)\left[2 \frac{\partial u}{\partial x} m 1_{x} n_{x}+2 \frac{\partial u}{\partial y} m 1_{y} n_{y}+2 \frac{\partial w}{\partial z} m 1_{z} n_{z}+\left(\frac{\partial u}{\partial y}+\frac{\partial v}{\partial x}\right)\left(m 1_{x} n_{y}+\right.\right. \\
\left.\left.m 1_{y} n_{x}\right)+\left(\frac{\partial u}{\partial z}+\frac{\partial w}{\partial x}\right)\left(m 1_{x} n_{z}+m 1_{z} n_{x}\right)+\left(\frac{\partial v}{\partial z}+\frac{\partial w}{\partial y}\right)\left(m 1_{y} n_{z}+m 1_{z} n_{y}\right)\right]=0, \\
\frac{1}{R e}\left(1+\nu_{t}\right)\left[2 \frac{\partial u}{\partial x} m 2_{x} n_{x}+2 \frac{\partial u}{\partial y} m 2_{y} n_{y}+2 \frac{\partial w}{\partial z} m 2_{z} n_{z}+\left(\frac{\partial u}{\partial y}+\frac{\partial v}{\partial x}\right)\left(m 2_{x} n_{y}+\right.\right. \\
\left.\left.m 2_{y} n_{x}\right)+\left(\frac{\partial u}{\partial z}+\frac{\partial w}{\partial x}\right)\left(m 2_{x} n_{z}+m 2_{z} n_{x}\right)+\left(\frac{\partial v}{\partial z}+\frac{\partial w}{\partial y}\right)\left(m 2_{y} n_{z}+m 2_{z} n_{y}\right)\right]=0 .
\end{gathered}
$$

Para o caso de escoamentos não newtonianos (viscoelásticos), as equações para velocidade e pressão na superfície livre são dadas por

$$
\begin{aligned}
& p= n_{x}^{2} \tau_{p_{x x}}+n_{y}^{2} \tau_{p_{y y}}+n_{z}^{2} \tau_{p_{z z}}+2\left(n_{x} n_{y} \tau_{p_{x y}}+n_{x} n_{z} \tau_{p_{x z}}+n_{y} n_{z} \tau_{p_{y z}}\right)+\frac{2 \vartheta}{R e}\left[n_{x}^{2} \frac{\partial u}{\partial x}\right. \\
&+\left.n_{y}^{2} \frac{\partial v}{\partial y}+n_{z}^{2} \frac{\partial w}{\partial z}+n_{x} n_{y}\left(\frac{\partial u}{\partial y}+\frac{\partial v}{\partial x}\right)+n_{x} n_{z}\left(\frac{\partial u}{\partial z}+\frac{\partial w}{\partial x}\right)+n_{y} n_{z}\left(\frac{\partial v}{\partial z}+\frac{\partial w}{\partial y}\right)\right], \\
& \frac{2 \vartheta}{R e}\left[n_{x} m_{1 x} \frac{\partial u}{\partial x}+n_{y} m_{1 y} \frac{\partial v}{\partial y}+n_{z} m_{1 z} \frac{\partial w}{\partial z}+\left(n_{x} m_{1 y}+n_{y} m_{1 x}\right)\left(\frac{\partial u}{\partial y}+\frac{\partial v}{\partial x}\right)\right. \\
&+\left.\left(n_{x} m_{1 z}+n_{z} m_{1 x}\right)\left(\frac{\partial u}{\partial z}+\frac{\partial w}{\partial x}\right)+\left(n_{y} m_{1 z}+n_{z} m_{1 y}\right)\left(\frac{\partial v}{\partial z}+\frac{\partial w}{\partial y}\right)\right] \\
&=-\left[n_{x} m_{1 x} \tau_{p} x x+n_{y} m_{1 y} \tau_{p_{y y}}+n_{z} m_{1 z} \tau_{p} z z\right. \\
&\left.+\left(n_{x} m_{1 y}+n_{y} m_{1 x}\right) \tau_{p_{x y}}+\left(n_{x} m_{1 z}+n_{z} m_{1 x}\right) \tau_{p_{x z}}+\left(n_{y} m_{1 z}+n_{z} m_{1 y}\right) \tau_{p} y z\right], \\
& \frac{2 \vartheta}{R e} {\left[n_{x} m_{2 x} \frac{\partial u}{\partial x}+n_{y} m_{2 y} \frac{\partial v}{\partial y}+n_{z} m_{2 z} \frac{\partial w}{\partial z}+\left(n_{x} m_{2 y}+n_{y} m_{2 x}\right)\left(\frac{\partial u}{\partial y}+\frac{\partial v}{\partial x}\right)\right.} \\
&+\left.\left(n_{x} m_{2 z}+n_{z} m_{2 x}\right)\left(\frac{\partial u}{\partial z}+\frac{\partial w}{\partial x}\right)+\left(n_{y} m_{2 z}+n_{z} m_{2 y}\right)\left(\frac{\partial v}{\partial z}+\frac{\partial w}{\partial y}\right)\right] \\
&=-\left[n_{x} m_{2 x} \tau_{p} x x+n_{y} m_{2 y} \tau_{p_{y y}}+n_{z} m_{2 z} \tau_{p} z z\right. \\
&\left.+\left(n_{x} m_{2 y}+n_{y} m_{2 x}\right) \tau_{p_{x y}}+\left(n_{x} m_{2 z}+n_{z} m_{2 x}\right) \tau_{p_{x z}}+\left(n_{y} m_{2 z}+n_{z} m_{2 y}\right) \tau_{p_{y z}}\right] .
\end{aligned}
$$

Para a implementação computacional, as discretizações das Eqs. (4.121)-(4.126) são feitas usando diferenças finitas considerando as diferentes orientações da superfície livre definidas em Tomé et al. (2001). Para ilustrar a aplicação dessas condições de contorno, considera-se o caso em que a superfície livre do fluido é aproximada por uma superfície planar 2D (com vetor normal fazendo $45^{0}$ com a direção do eixo coordenado $x$ ), como mostrado na Fig. 4.3. Para esta representação particular da superfície livre local, os vetores tangenciais e normal são tomados como $\mathbf{m}_{\mathbf{1}}=\left(\frac{\sqrt{2}}{2}, 0,-\frac{\sqrt{2}}{2}\right), \mathbf{m}_{\mathbf{2}}=(0,1,0)$ e 
$\mathbf{n}=\left(\frac{\sqrt{2}}{2}, 0, \frac{\sqrt{2}}{2}\right)$; este é o caso de uma célula $\mathbf{S}$ ter as faces $\left(k+\frac{1}{2}\right)$ e $\left(i+\frac{1}{2}\right)$ em contato com uma célula E. Levando esses vetores nas equações (4.121), (4.122) e (4.123) obtém-se as equações simplificadas

$$
p_{e}=\frac{2}{R e}\left(1+\nu_{t}\right)\left(\frac{\partial u}{\partial x}+\frac{\partial w}{\partial z}+\frac{\partial u}{\partial z}+\frac{\partial w}{\partial x}\right)
$$

e

$$
\frac{1}{R e}\left(1+\nu_{t}\right)\left[\left(\frac{\partial u}{\partial x}-\frac{\partial w}{\partial z}\right)\right]=0
$$

As velocidades $u_{i+\frac{1}{2}, j, k}$ e $w_{i, j, k+\frac{1}{2}}$ (ver Fig. 4.3) são obtidas usando-se a equação da continuidade (4.79) e a Eq. (4.128), ambas avaliadas no ponto $\mathcal{P}=(i, j, k)$ e discretizadas, respectivamente, como

$$
\frac{u_{i+\frac{1}{2}, j, k}-u_{i-\frac{1}{2}, j, k}}{\delta x}+\frac{v_{i, j+\frac{1}{2}, k}-v_{i, j-\frac{1}{2}, k}}{\delta y}+\frac{w_{i, j, k+\frac{1}{2}}-w_{i, j, k-\frac{1}{2}}}{\delta z}=0
$$

e

$$
\frac{1}{R e}\left(1+\nu_{i, j, k}\right)\left(\frac{u_{i+\frac{1}{2}, j, k}-u_{i-\frac{1}{2}, j, k}}{\delta x}-\frac{w_{i, j, k+\frac{1}{2}}-w_{i, j, k-\frac{1}{2}}}{\delta z}\right)=0 .
$$

Dessas duas equações discretas calculam-se as velocidades $u_{i+\frac{1}{2}, j, k}$ e $w_{i, j, k+\frac{1}{2}}$, cujos resultados são

$$
u_{i+\frac{1}{2}, j, k}=u_{i-\frac{1}{2}, j, k}-\frac{1}{2} \frac{\delta x}{\delta y}\left(v_{i, j+\frac{1}{2}, k}-v_{i, j-\frac{1}{2}, k}\right)
$$

e

$$
w_{i, j, k+\frac{1}{2}}=w_{i, j, k-\frac{1}{2}}-\frac{1}{2} \frac{\delta z}{\delta y}\left(v_{i, j+\frac{1}{2}, k}-v_{i, j-\frac{1}{2}, k}\right) .
$$

De posse das velocidades $u_{i+\frac{1}{2}, j, k}$ e $w_{i, j, k+\frac{1}{2}}$, a pressão $p_{e}$ é calculada via a discretização da Eq. (4.127) no ponto $\mathcal{P}=(i, j, k)$; o resultado é

$$
\begin{aligned}
p_{e_{i, j, k}}=\frac{1}{R e}(1+ & \left.\nu_{t_{i, j, k}}\right)\left\{\frac{u_{i+\frac{1}{2}, j, k}-u_{i-\frac{1}{2}, j, k}}{\delta x}+\frac{w_{i, j, k+\frac{1}{2}}-w_{i, j, k-\frac{1}{2}}}{\delta z}\right. \\
& +\frac{1}{2}\left(\frac{u_{i+\frac{1}{2}, j, k}-u_{i-\frac{1}{2}, j, k}-u_{i+\frac{1}{2}, j, k-1}-u_{i-\frac{1}{2}, j, k-1}}{\delta z}\right. \\
& \left.\left.\frac{w_{i, j, k+\frac{1}{2}}+w_{i, j, k-\frac{1}{2}}-w_{i-1, j, k+\frac{1}{2}}-w_{i-1, j, k-\frac{1}{2}}}{\delta x}\right)\right\} .
\end{aligned}
$$

A aplicação das condições de contorno na superfície livre para os outros casos é feita de maneira análoga ao caso descrito anteriormente.

A condição de Neumann homogênea é adotada para as variáveis turbulentas, $\kappa$ e $\varepsilon$, e contribuição não newtoniana $\boldsymbol{\tau}_{p}$, na superfície livre, isto é $\frac{\partial \phi}{\partial \mathbf{n}} \approx \frac{\partial \phi}{\partial \mathfrak{a}}$, onde $\phi$ é uma das variáveis $\kappa, \varepsilon, \tau_{p_{x x}}, \tau_{p_{x y}}$, $\tau_{p_{x z}}, \tau_{p_{y y}}, \tau_{p_{y z}}, \tau_{p_{x x}}, \tau_{p_{z z}}$ e $\mathfrak{a}=x, y$ ou $z$. A discretização dessa condição é feita usando diferença avançada ou atrasada de primeira ordem.

\subsubsection{Na superfície rígida}

A condição de contorno adotada na superfície rigida para o caso de escoamentos newtonianos no regime laminar é a condição de não escorregamento $(\mathbf{u}=0)$ ou deslizamento $(\mathbf{u} \cdot \mathbf{n}=0)$. 


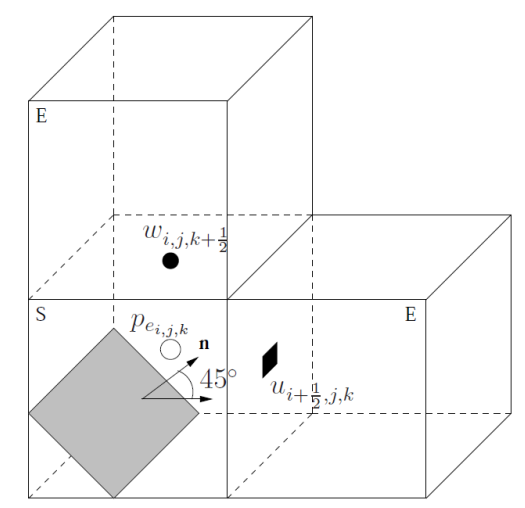

Figura 4.3: Ilustração de uma superfície planar inclinada formando angulo de $45^{0} \operatorname{com} \mathbf{n}=\left(\frac{\sqrt{2}}{2}, 0, \frac{\sqrt{2}}{2}\right)$. Extraído de Kurokawa (2009).

Para escoamentos newtonianos no regime turbulento adota-se uma modificação das leis de parede de Sondak e Pletcher (1995) numa região próxima no contorno. A viscosidade turbulenta é calculada via a função de amortecimento de van Driest (1956) definida por

$$
\nu_{t}=K u_{\star} y\left(1-\exp \left(\frac{-y^{+}}{A}\right)\right)^{2}, \quad y^{+}=u_{\star} y / \nu, \quad A \approx 26,
$$

onde $K=0.41$ é a constante de von Kármán, $y^{+}=u_{\star} y / \nu, A \approx 26$ é o número de Reynolds local, e $u_{\star}$ a velocidade de atrito. Nessa região as variáveis turbulentas $\kappa$ e $\varepsilon$ são definidas segundo as subcamadas turbulenta e viscosa, isto é:

-na subcamada turbulenta, $\kappa$ e $\varepsilon$ são calculadas por

$$
\kappa=R e \frac{u_{\star}^{2}}{\sqrt{C_{\mu}}}, \quad \varepsilon=\operatorname{Re} \frac{u_{\star}^{3}}{\kappa y} .
$$

-na subcamada viscosa, a energia cinética turbulenta adimensionalizada $k$ é definida pela correção de Sondak e Pletcher (1995):

$$
\kappa=\operatorname{Re} \frac{u_{\star}^{2}}{\sqrt{C_{\mu}}}\left(\frac{y^{+}}{y_{c}^{+}}\right)^{2},
$$

onde $y_{c}^{+}$é o número de Reynolds local crítico, cujo valor é a solução da equação não linear resultante da interseção das leis

$$
u^{+}=\operatorname{Re}\left(y u_{\star}\right) \quad \text { e } \quad \ln \left(E R e\left(y u_{\star}\right)\right)-K \frac{u}{u_{\star}}=0,
$$

em que $E=\exp (K B)$. A ultima equação pode ser reescrita como:

$$
\ln \left(E y_{c}^{+}\right)-K y_{c}^{+}=0
$$

Na Eq. (4.136), u é a magnitude da velocidade tangencial à distância y da parede. As Eqs. (4.136)-(4.137) são as leis de parede usadas neste trabalho e são restritas segundo o valor do Reynolds local $y^{+}$. Essas 
leis são válidas para valores de $y^{+} \approx 5$ e aplicável no intervalo $30<y^{+}<300$ (McComb, 1990). A Fig. 4.4 mostra o perfil tipico de uma camada limite turbulenta. No caso de escoamentos newtonianos sobre superfícies suaves, a partir de dados experimentais de Bradshaw (1976), a constante $B$ está no intervalo $5.0 \leq B \leq 5.2$; o valor adotado aqui é $B=5$.

Considerando a escala de comprimento $l^{\star}$ (H. Lee Norris, 1975) definida por

$$
l^{\star}=\frac{K C_{\mu}^{-\frac{3}{4}} y}{1+5.3 / R e_{t}},
$$

com $R e_{t}=y \sqrt{R e k}$, o valor de $\varepsilon$ é definido por

$$
\varepsilon=\sqrt{\frac{1}{R e}} \frac{\kappa^{\frac{3}{2}}}{l^{\star}}
$$

A tensão de cisalhamento na parede (na forma adimensional) é dada por (Wilcox, 1994))

$$
\tau_{\omega}=\left.u^{\star^{2}} \approx\left(\frac{1}{R e}\left(1+\nu_{t}\right)\left|\frac{\partial u}{\partial n}\right|\right)\right|_{\text {wall }}
$$

em que $u$ e $n$ são a velocidade tangencial e a direção normal ao contorno rígido, respectivamente. A Eq. (4.140) é avaliada no contorno rígido. No entanto, fora do domínio da solução, a velocidade $u$ é avaliada discretizando a derivada por diferenças centrais, considerando que a velocidade tangencial tem sinal oposto à tensão efetiva (Ferreira et al., 2004). Mais detalhes sobre lei de parede veja, por exemplo, Bradshaw (1995), Morrison (2007) e Pope (2010).

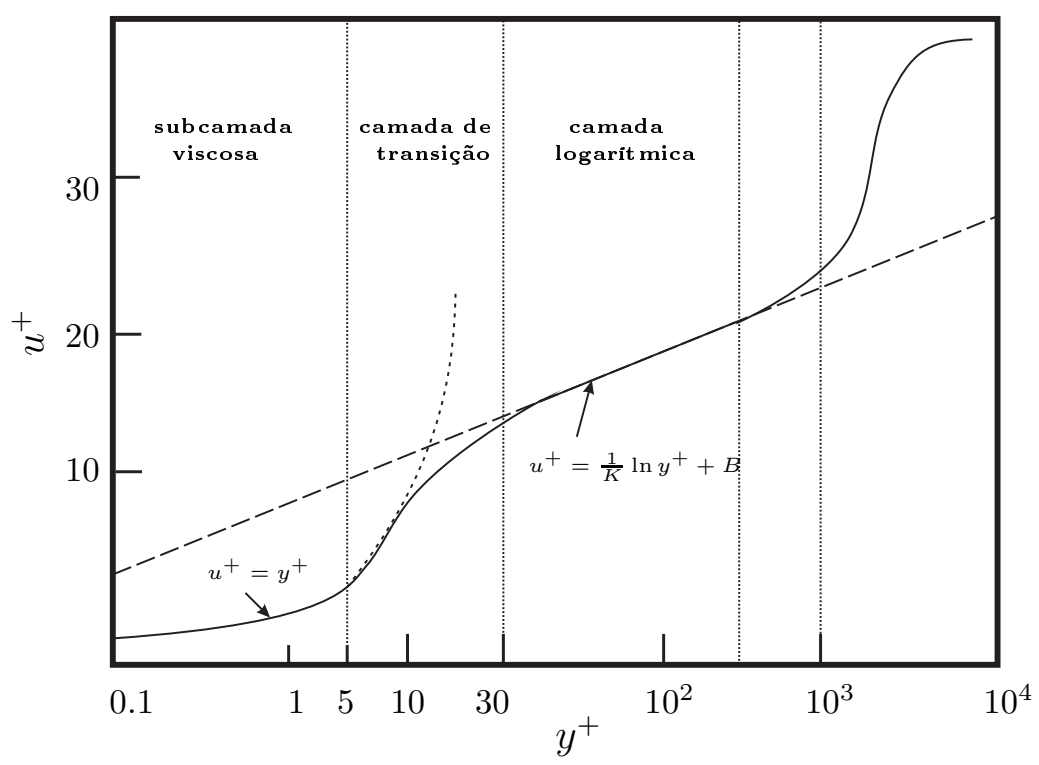

Figura 4.4: Perfil típico da camada limite turbulenta.

A seguir é apresentado um caso particular da discretização das condições de contorno para o caso turbulento, onde são definidas as velocidades tangenciais como $u$ e $w$ e uma variável genérica $\phi$ que pode ser uma das variáveis turbulentas $\kappa, \varepsilon$ ou $\nu_{t}$ (ver Fig. 4.5). Nesse trabalho, nas células adjacentes ao contorno rígido as equações médias de Reynolds são implementadas com o objetivo de se calcular as velocidades; e para as variáveis turbulentas é implementada a lei de parede. 


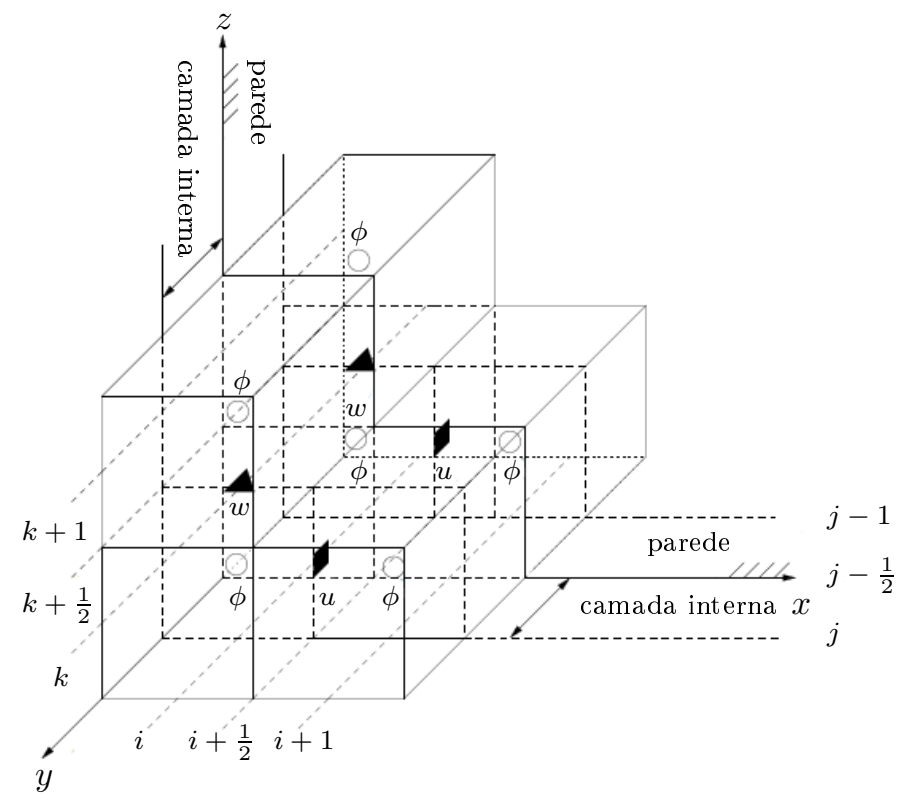

Figura 4.5: Ilustra-se a camada interna adjacente á parede e as células fictícias. Extraído de Kurokawa (2009).

As variáveis $\kappa$ e $\varepsilon$ são calculadas na subcamada turbulenta, avaliadas no ponto $(i, j, k)$, respectivamente, por

$$
\kappa_{i, j, k}=R e \frac{\tau_{w}}{\sqrt{C_{\mu}}} \text { e } \quad \varepsilon_{i, j, k}=R e \frac{\tau_{\omega} u_{\star}}{K y},
$$

onde a velocidade do atrito é calculada segundo o valor adotado por $y^{+}$. Na subcamada viscosa, essas variáveis são dadas por

$$
\kappa_{i, j, k}=R e \frac{\tau_{w}}{\sqrt{C_{\mu}}}\left(\frac{y^{+}}{y_{c}^{+}}\right)^{2} \quad \text { e } \varepsilon_{i, j, k}=\sqrt{\frac{1}{R e}} \frac{\left(\kappa_{i, j, k}\right)^{3 / 2}}{l^{\star}},
$$

com a escala de comprimento $l^{\star}$ definida por (4.138). A viscosidade turbulenta é definida por

$$
\nu_{t i, j, k}=C_{\mu} \frac{\kappa_{i, j, k}^{2}}{\varepsilon_{i, j, k}} .
$$

Fora do domínio computacional as velocidades, $u_{i+\frac{1}{2}, j-1, k+\frac{1}{2}}$ e $w_{i, j-1, k+\frac{1}{2}}$ são discretizadas por diferenças centrais (Wilcox, 1994) a partir de (4.140) nos pontos $\left(i+\frac{1}{2}, j-\frac{1}{2}, k\right)$ e $\left(i, j-\frac{1}{2}, k+\frac{1}{2}\right)$. A velocidade tangencial $u$ é calculada no ponto $\left(i+\frac{1}{2}, j-1, k\right)$ como

$$
u_{i+\frac{1}{2}, j-1, k}=u_{i+\frac{1}{2}, j, k}-\operatorname{signal}\left(u_{i+\frac{1}{2}, j, k}\right) \frac{\operatorname{Re} \tau_{\omega} \delta y}{\left(1+\nu_{t_{i+\frac{1}{2}, j-\frac{1}{2}, k}}\right)} .
$$

$\operatorname{signal}\left(u_{i+\frac{1}{2}, j, k}\right)$ é a sinal da velocidade tangencial. A viscosidade turbulenta é definida por

$$
\nu_{t_{i+\frac{1}{2}, j-\frac{1}{2}, k}}=0.25\left(\nu_{t_{i, j-1, k}}+\nu_{t_{i, j, k}}+\nu_{t_{i+1, j-1, k}}+\nu_{t_{i+1, j, k}}\right) .
$$


A velocidade $w$ avaliada no ponto $\left(i, j-\frac{1}{2}, k+\frac{1}{2}\right)$ é calculada como

$$
w_{i, j-1, k+\frac{1}{2}}=w_{i, j, k+\frac{1}{2}}-\operatorname{signal}\left(w_{i, j, k+\frac{1}{2}}\right) \frac{\operatorname{Re} \tau_{\omega} \delta y}{\left(1+\nu_{t_{i, j-\frac{1}{2}, k+\frac{1}{2}}}\right)}
$$

Nesse caso a viscosidade turbulenta $\nu_{t}$ é dada por

$$
\nu_{t_{i, j-\frac{1}{2}, k+\frac{1}{2}}}=0.25\left(\nu_{t_{i, j-1, k}}+\nu_{t_{i, j, k}}+\nu_{t_{i, j-1, k}}+\nu_{t_{i, j, k+1}}\right) .
$$

Nas Eqs. (4.141)-(4.144) a velocidade de atrito $u_{\star}$ é calculada, usando as equações em (4.136) dependendo do valor do $y^{+}$em (4.133) pelo método de Newton-Raphson com $u_{\star}=11.6$ com aproximação inicial e critério de parada $1.0 \times 10^{-14}$. Os cálculos são inicializados calculando-se o número de Reynolds critico $y_{c}^{+}$é admitindo que $y^{+}$está na camada viscosa.

Para escoamentos não newtonianos (viscoelásticos) as condições de contorno na superfície rígida adotadas nesse trabalho foram não deslizamento. Para a contribuição não newtoniana existem três casos a considerar, cujos detalhes podem ser encontrados em Figueiredo (2011): quando os contornos rígidos são paralelos ao plano $x y$, as condições de contorno adotadas para a contribuição não newtoniana $\boldsymbol{\tau}_{p}$ são calculadas a partir das condições de impermeabilidade e não deslizamento,

$$
u=v=w=0 \Rightarrow \frac{\partial u}{\partial x}=\frac{\partial v}{\partial x}=\frac{\partial w}{\partial x}=0 \quad \text { e } \quad \frac{\partial u}{\partial y}=\frac{\partial v}{\partial y}=\frac{\partial w}{\partial y}=0
$$

e usando a equação da continuidade tém-se que

$$
\frac{\partial u}{\partial x}=\frac{\partial v}{\partial y}=0 \quad \Rightarrow \quad \frac{\partial w}{\partial z}=0 .
$$

Com esse duas últimas equações e a Eq. (4.81), segue as condições para o contorno rígido para a contribuição não newtoniana $\boldsymbol{\tau}_{p}$

$$
\begin{aligned}
& \frac{\partial \tau_{p_{x x}}}{\partial t}=2 \frac{\partial u}{\partial z} \tau_{p_{x z}}-\frac{1}{W e}\left\{f\left(\lambda, \tau_{p}\right) \tau_{p_{x x}}+\Upsilon\left(f\left(\lambda, \tau_{p}\right)-1\right)+\frac{\varrho}{\Upsilon}\left[\left(\tau_{p_{x x}}\right)^{2}+\left(\tau_{p_{x y}}\right)^{2}+\left(\tau_{p_{x z}}\right)^{2}\right]\right\}, \\
& \frac{\partial \tau_{p_{y y}}}{\partial t}=2 \frac{\partial v}{\partial z} \tau_{p_{y z}}-\frac{1}{W e}\left\{f\left(\lambda, \tau_{p}\right) \tau_{p_{y y}}+\Upsilon\left(f\left(\lambda, \tau_{p}\right)-1\right)+\frac{\varrho}{\Upsilon}\left[\left(\tau_{p_{x y}}\right)^{2}+\left(\tau_{p_{y y}}\right)^{2}+\left(\tau_{p_{y z}}\right)^{2}\right]\right\}, \\
& \frac{\partial \tau_{p_{z z}}}{\partial t}=-\frac{1}{W e}\left\{f\left(\lambda, \tau_{p}\right) \tau_{p_{z z}}+\Upsilon\left(f\left(\lambda, \tau_{p}\right)-1\right)+\frac{\varrho}{\Upsilon}\left[\left(\tau_{p_{x z}}\right)^{2}+\left(\tau_{p_{y z}}\right)^{2}+\left(\tau_{p_{z z}}\right)^{2}\right]\right\}, \\
& \frac{\partial \tau_{p_{x y}}}{\partial t}=\frac{\partial u}{\partial z} \tau_{p_{y z}}+\frac{\partial v}{\partial z} \tau_{p_{x z}}-\frac{1}{W e}\left\{f\left(\lambda, \tau_{p}\right) \tau_{p_{x y}}+\frac{\varrho}{\Upsilon}\left[\tau_{p_{x x}} \tau_{p_{x y}}+\tau_{p_{y y}} \tau_{p_{x y}}+\tau_{p_{x z}} \tau_{p_{y z}}\right]\right\}, \\
& \frac{\partial \tau_{p_{x z}}}{\partial t}=\frac{\partial u}{\partial z}\left(\tau_{p_{z z}}+\Upsilon\right)-\frac{1}{W e}\left\{f\left(\lambda, \tau_{p}\right) \tau_{p_{x z}}+\frac{\varrho}{\Upsilon}\left[\tau_{p_{x x}} \tau_{p_{x z}}+\tau_{p_{x y}} \tau_{p_{y z}}+\tau_{p_{x z}} \tau_{p_{z z}}\right]\right\}, \\
& \frac{\partial \tau_{p_{y z}}}{\partial t}=\frac{\partial v}{\partial z}\left(\tau_{p_{z z}}+\Upsilon\right)-\frac{1}{W e}\left\{f\left(\lambda, \tau_{p}\right) \tau_{p_{y z}}+\frac{\varrho}{\Upsilon}\left[\tau_{p_{x y}} \tau_{p_{x z}}+\tau_{p_{y y}} \tau_{p_{y z}}+\tau_{p_{y z}} \tau_{p_{z z}}\right]\right\} .
\end{aligned}
$$

Nos outros dois casos, quando consideram-se contornos rígidos paralelos ao plano $x z$ e $y z$, as aproximações na superfície rigida é feita de forma similar que para o caso $x y$ usando as condições de impermeabilidade e aderência completa (Figueiredo, 2011). 


\section{Capítulo}

\section{Resultados numéricos para sistemas hiperbólicos $1 D$ e $2 D$}

Neste capítulo são apresentados novos resultados numéricos para sistemas hiperbólicos de leis de conservação fazendo-se o uso do esquema de convecção TDPUS-C3 com o parâmetro livre $\beta$ escolhido com base em advecção de escalares e a equação não linear de Burgers. Comparações dos resultados fornecidos pelo esquema TDPUS-C3 com resultados obtidos de esquemas bem estabelecidos na literatura, tais como VAN LEER por van Leer (1974), MC por van Leer (1977), MINMOD por Roe (1986), SUPERBEE por Roe (1986), ARORA-ROE por Arora e Roe (1997), Godunov-HLL por Toro (1999), CUBISTA por Alves et al. (2003), ADBQUICKEST por Ferreira et al. (2009), FORCE por Stecca et al. (2010), TOPUS por Ferreira et al. (2012), SDPUS-C1 por Lima et al. (2012) e EPUS por Corrêa et al. (2012), são também apresentadas. O propósito é mostrar que o esquema TDPUS-C3 atinge ao menos segunda ordem de precisão local, é útil em resolver descontinuidades/choques (sem gerar oscilações e com pouca introdução de viscosidade numérica), e consegue capturar estruturas complexas em escoamentos de gases e plasmas. Para a simulação computacional, dois códigos foram utilizados: (i) um código próprio, em diferenças finitas, implementado em $\mathrm{C}++$ para simular advecção de escalares e equações não lineares de Burguers e Buckley-Leverett (no apêndice B encontra-se a implementação da equação de Burgers 2D); e (ii) o código CLAWPACK (LeVeque, 2004) implementado em volumes finitos para simular sistemas não lineares tais como equações de Euler (em LeVeque (2004) encontra-se uma descrição detalhada da implementação desse código).

\subsection{Escolha do parâmetro livre $\beta$ para o esquema TDPUS-C3}

A determinação do parâmetro livre $\beta$ para o esquema TDPUS-C3, tanto em variáveis normalizadas Eq. (2.55) como em limitador de fluxo Eq. (2.58), é feita inicialmente utilizando o transporte linear da combinação de quatro tipos de ondas (como condição inicial) via equação de advecção 1D no domínio 
$[-1,1]$ (ver Zalesak (1987)), isto é

$$
u_{0}(x)= \begin{cases}\frac{1}{6}\left[G\left(x, \beta, z-\delta_{1}\right)+G\left(x, \beta, z+\delta_{1}\right)+4 G\left(x, \beta_{1}, z\right)\right], & -0.8 \leq x \leq-0.6 \\ 1, & -0.4 \leq x \leq-0.2 \\ 1-|10(x-0.1)|, & 0.0 \leq x \leq 0.2 \\ \frac{1}{6}\left[F\left(x, \alpha_{1}, a_{1}-\delta_{1}\right)+F\left(x, \alpha_{1}, a_{1}+\delta_{1}\right)+4 F\left(x, \alpha_{1}, a_{1}\right)\right], & 0.4 \leq x \leq 0.6, \\ 0, \text { caso contrário, } & \end{cases}
$$

onde $G\left(x, \beta_{1}, z\right)=e^{\beta_{1}(x-z)^{2}}, F\left(x, \alpha_{1}, a_{1}\right)=\sqrt{\max \left(1-\alpha_{1}^{2}\left(x-\alpha_{1}\right)^{2}, 0\right)}, a_{1}=0.5, z=-0.7, \alpha_{1}=10$, $\beta_{1}=\frac{\log 2}{36 \delta_{1}^{2}}, \delta_{1}=0.005$. A função fluxo neste teste é $f(u)=a u$ com a velocidade de convecção $a=1$. Utilizaram-se neste teste condição de contorno periódica e os seguintes dados: malha computacional de 200 células, número de $C F L=0.5$ e tempo final de simulação $t=1$. Para a simulação do problema utilizando-se o código próprio incrementado com o esquema TDPUS-C3 foram considerados os cinco valores de $\beta$ da Tab. 2.2. As simulações numéricas com os vários valores do parâmetro $\beta$ estão apresentadas na Fig. 5.1, onde pode-se constatar que o esquema TDPUS-C3 com o $\beta=567.25$, quando comparado com a solução exata, forneceu o melhor resultado. Nota-se também por essa mesma figura que, da mesma forma como acontece com a maioria dos esquemas de alta resolução, o esquema TDPUS-C3 apresenta o problema de aparamentos de picos e vales.

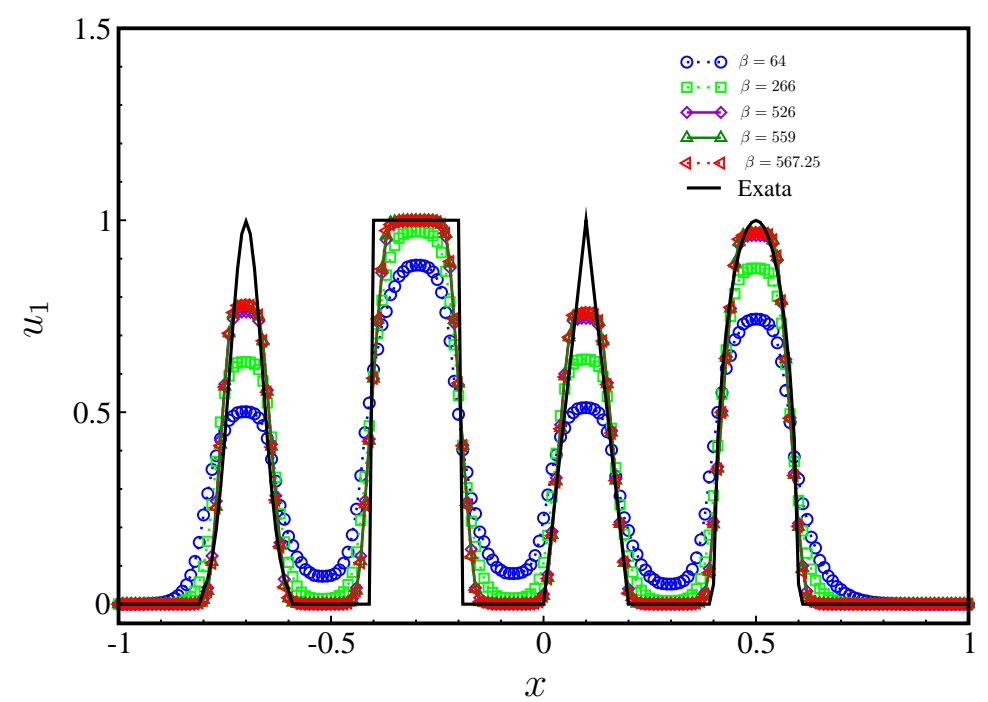

Figura 5.1: Escolha do parâmetro $\beta$. Soluções numéricas calculadas com TDPUS-C3 e $\beta=64,266$, $526,567.25$ para o problema de advecção do teste de Zalesak. $t=1, N=200$ e $C F L=0.5$.

Com o objetivo de confirmar que o esquema TDPUS-C3, com $\beta=567.25$, fornece também os melhores resultados em problemas não lineares, escolheu-se a equação 1D (3.1) no domínio [-1,1] com a função fluxo $0.5 u^{2}$ (equação de Burgers). Para tanto, o transporte não linear da condição inicial $u_{1}(x, 0)=1+0.5 \operatorname{sen}(\pi \mathrm{x})$ via a equação de Burgers $1 \mathrm{D}$ sem coeficiente de difusão foi considerado. 
Para a simulação do problema, tomaram-se quatro malhas computacionais $(N=40,80,160$ e 320), $C F L=0.5$ e tempo final $t=0.33$. Para estimar a ordem de convergência observada na norma $L_{1}$ do método numérico global equipado com o esquema TDPUS-C3, a solução numérica na malha 640 foi tomada como referência. Na Tab. 5.1 observa-se uma melhor estimativa da ordem de convergência para o valor $\beta=567.25$.

Tabela 5.1: Escolha do parâmetro $\beta$. Teste de convergência para TDPUS-C3 com $\beta=64,266,526$ e 567.25 da equação de Burgers $1 \mathrm{D}$ no tempo $t=0.33$ e $C F L=0.5$.

\begin{tabular}{|c|c|c|c|c|c|c|c|c|c|c|}
\hline & \multicolumn{2}{|l|}{$\beta=64$} & \multicolumn{2}{|c|}{$\beta=266$} & \multicolumn{2}{|l|}{$\beta=526$} & \multicolumn{2}{|c|}{$\beta=559$} & \multicolumn{2}{|c|}{$\beta=567.25$} \\
\hline Malha & $L_{1}$ & $q$ & $L_{1}$ & $q$ & $L_{1}$ & $q$ & $L_{1}$ & $q$ & $L_{1}$ & $q$ \\
\hline 40 & $9.00 \times 10^{-}$ & 1.97 & $7.94 \times 10^{-3}$ & 1.93 & $.73 \times 10^{-3}$ & 1.93 & $81 \times 10$ & 1.96 & $8.83 \times 10^{-2}$ & 1.97 \\
\hline 80 & $4.16 \times 10^{-3}$ & 1.88 & $3.54 \times 10^{-3}$ & 2.09 & $3.54 \times 10^{-3}$ & 2.28 & $3.58 \times 10^{-3}$ & 2.30 & $3.59 \times 10^{-3}$ & 2.30 \\
\hline 160 & $1.77 \times 10^{-}$ & 2.11 & $1.66 \times 10^{-3}$ & 2.07 & $1.67 \times 10^{-3}$ & 2.12 & $1.68 \times 10^{-3}$ & 2.13 & $1.69 \times 10^{-3}$ & 2.14 \\
\hline 320 & $8.43 \times 10^{-4}$ & 2.06 & $8.04 \times 10^{-4}$ & 2.04 & $8.00 \times 10^{-4}$ & 2.08 & $8.02 \times 10^{-4}$ & 2.08 & $8.03 \times 10^{-4}$ & 2.09 \\
\hline
\end{tabular}

É importante observar neste ponto que, em função dos resultados obtidos para os dois testes precedentes (um linear e outro não linear), utilizar-se-á nas simulações o esquema TDPUS-C3 com parâmetro livre $\beta=567.25$.

\subsection{Equações 1D e 2D de leis conservação}

Nesta seção são apresentados resultados numéricos com o esquema TDPUS-C3 para equações de conservação 1D. São apresentadas também comparações dos resultados obtidos com o TDPUS-C3 e os resultados com os esquemas ADBQUICKEST, ARORA-ROE, CUBISTA, TOPUS, SDPUS-C1 e EPUS.

\subsubsection{Advecção de escalares}

Advecção de escalares é o problema mais simples em DFC e modelado pela Eq. (3.1) com função fluxo $f(u)=a u_{1}, a$ constante. Dois casos teste são simulados nesta seção com o uso dos esquemas convectivos: (Teste 1) o transporte da condição inicial em pequenos e longos tempos de simulação; e (Teste 2) o transporte de $u_{1}(x, 0)=\sin ^{4} \pi x$ para $x \in[-1,1]$. O Teste 1 serve para verificar se o esquema numérico consegue simular uma combinação de várias ondas de diferentes tipos; e o Teste 2 tem por objetivo investigar o comportamento assintótico do erro quando a malha é refinada.

Para a solução numérica do Teste 1 foram utilizados condições de contorno periódicas, tempos finais de simulação $t=1$ e $t=20$, malha de $N=200$ células computacionais e número de Courant $C F L=0.5$. Os resultados obtidos estão descritos nas Figs. 5.2, para $t=1$, e 5.3, para $t=20$, onde pode-se observar que para pequenos tempos o esquema TDPUS-C3 forneceu o melhor resultado; enquanto que, para tempos longos, o esquema TDPUS-C3 forneceu resultados inferiores aos fornecidos pelos esquemas CUBISTA e TOPUS e superiores aos dos outros esquemas. Observa-se ainda por essas figuras que ambos os esquemas ADBQUICKEST e ARORA-ROE suavizam sobremaneira a solução aproximada. As Tabs. 5.2 e 5.3 apresentam nesses tempos finais os erros e as ordens de convergência observadas na norma $L_{1}$ do método numérico global equipado com os vários esquemas de convecção. Os dados nessas tabelas quantificam os resultados apresentados nas Figs. 5.2 e 5.3. 


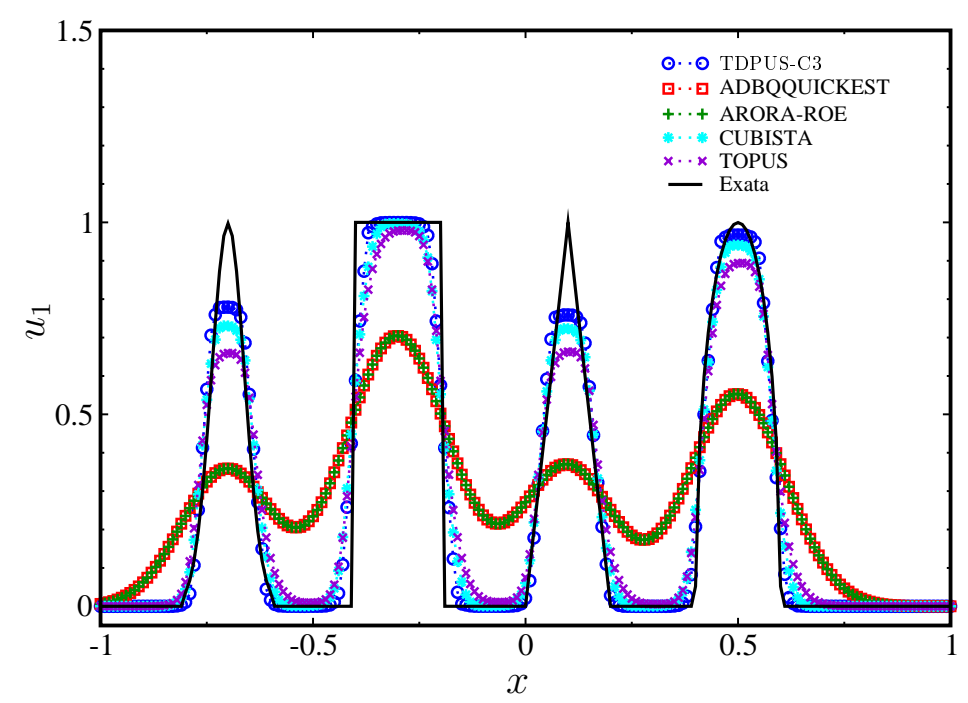

Figura 5.2: Teste 1: soluções numéricas do problema de advecção 1D com TDPUS-C3, ADBQUICKEST, ARORA-ROE, CUBISTA e TOPUS. $t=1, N=200$ e $C F L=0.5$.

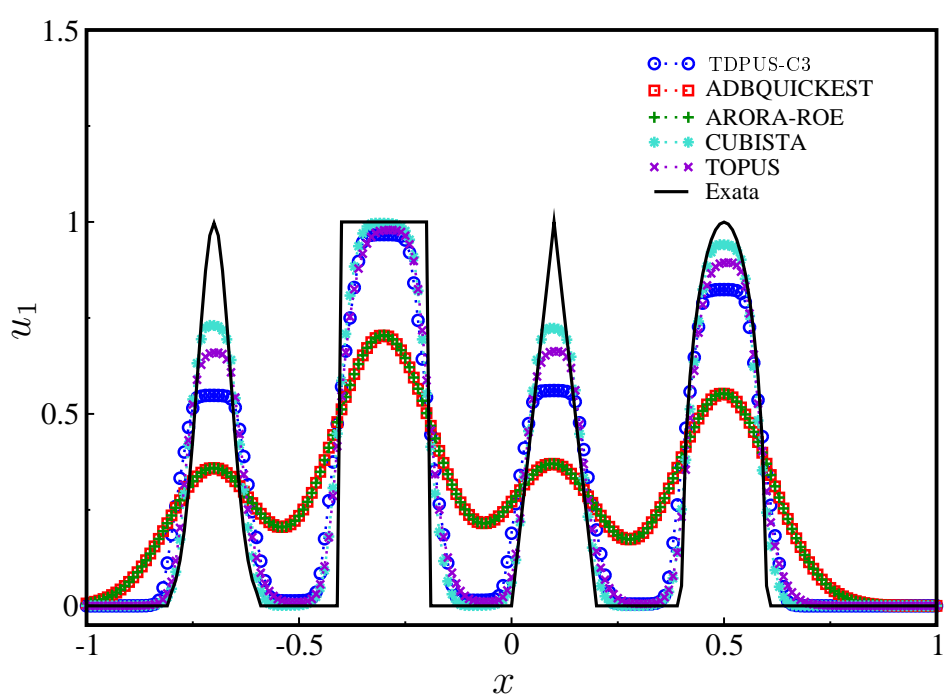

Figura 5.3: Teste 1: soluções numéricas do problema de advecção 1D com TDPUS-C3, ADBQUICKEST, ARORA-ROE, CUBISTA e TOPUS. $t=20, N=200$ e $C F L=0.5$. 
Tabela 5.2: Teste 1: teste de convergência do experimento de Zalesak com TDPUS-C3, ADBQUICKEST, ARORA-ROE, CUBISTA e TOPUS no tempo $t=1$. SSP-RK3 na parte temporal.

\begin{tabular}{ccccccccccc}
\hline \hline & \multirow{2}{*}{ TDPUS-C3 } & \multicolumn{2}{c}{ ADBQUICKEST } & \multicolumn{2}{c}{ ARORA-ROE } & \multicolumn{2}{c}{ CUBISTA } & \multicolumn{2}{c}{ TOPUS } \\
\hline Malha & $L_{1}$ & $q$ & $L_{1}$ & $q$ & $L_{1}$ & $q$ & $L_{1}$ & $q$ & $L_{1}$ & $q$ \\
\hline 200 & $7.87 E-02$ & - & $4.50 E-01$ & - & $4.50 E-01$ & - & $1.04 E-01$ & - & $1.40 E-01$ & - \\
400 & $4.32 E-02$ & 0.86 & $3.24 E-01$ & 0.47 & $3.24 E-01$ & 0.47 & $5.40 E-02$ & 0.95 & $7.01 E-02$ & 1.00 \\
800 & $2.04 E-02$ & 1.08 & $2.19 E-01$ & 0.56 & $2.19 E-01$ & 0.56 & $2.70 E-02$ & 1.00 & $3.62 E-02$ & 0.95 \\
1600 & $1.03 E-02$ & 0.98 & $1.42 E-01$ & 0.63 & $1.42 E-01$ & 0.63 & $1.43 E-02$ & 0.91 & $1.95 E-02$ & 0.89 \\
\hline \hline
\end{tabular}

Tabela 5.3: Teste 1: teste de convergência do experimento de Zalesak com TDPUS-C3, ADBQUICKEST, ARORA-ROE, CUBISTA e TOPUS no tempo $t=20$. SSP-RK3 na parte temporal.

\begin{tabular}{ccccccccccc}
\hline \hline & \multirow{2}{*}{ TDPUS-C3 } & \multicolumn{2}{c}{ ADBQUICKEST } & \multirow{2}{*}{ ARORA-ROE } & \multicolumn{2}{c}{ CUBISTA } & \multicolumn{2}{c}{ TOPUS } \\
\hline Malha & $L_{1}$ & $q$ & $L_{1}$ & $q$ & $L_{1}$ & $q$ & $L_{1}$ & $q$ & $L_{1}$ & $q$ \\
\hline 200 & $1.84 E-01$ & - & $6.42 E-01$ & - & $6.43 E-01$ & - & $2.97 E-01$ & - & $4.03 E-1$ & - \\
400 & $7.74 E-02$ & 1.25 & $6.26 E-01$ & 0.04 & $6.26 E-01$ & 0.04 & $1.29 E-01$ & 1.20 & $1.75 E-1$ & 1.20 \\
800 & $4.51 E-02$ & 0.78 & $5.87 E-01$ & 0.09 & $5.86 E-01$ & 0.09 & $6.80 E-02$ & 0.92 & $8.19 E-2$ & 1.10 \\
1600 & $2.56 E-02$ & 0.81 & $4.96 E-01$ & 0.24 & $4.95 E-01$ & 0.24 & $3.70 E-02$ & 0.88 & $4.29 E-2$ & 0.93 \\
\hline \hline
\end{tabular}

A Tab. 5.4 apresenta tempos de computação (custo) em milissegundo ( $\mu s$ ) por iteração (e normalizado com respeito ao mais barato) para o Teste 1 usando a malha $N=200$. Vê-se por essa tabela que o custo para o esquema TDPUS-C3 é menor que os custos correspondentes para os esquemas ADBQUICKEST e ARORA-ROE, e próximo dos custos para os esquemas CUBISTA e TOPUS.

Tabela 5.4: Tempo de computação por iteração para o Teste 1.

\begin{tabular}{ccc}
\hline \hline Esquema & Custo por iteração $(\mu \mathrm{s})$ & Custo normalizado \\
\hline TDPUS-C3 & 27.68 & 1.11 \\
ADBQUICKEST & 29.53 & 1.18 \\
ARORA-ROE & 46.59 & 1.86 \\
CUBISTA & 25.04 & 1.00 \\
TOPUS & 26.37 & 1.05 \\
\hline \hline
\end{tabular}

Para investigar o comportamento assintótico do erro em advecção de um escalar quando $\delta x \rightarrow 0$ (objetivo do Teste 2), transporta-se o perfil inicial $u_{1}(x, 0)=\sin ^{4} \pi x$ usando as nove malhas de tamanho $N=20,40,80,160,320,640,1280,2560$ e 5120 células computacionais, no tempo final $t=1$, número de Courant $C F L=0.5$ e com condições de contorno periódicas. Na Tab. 5.5 estão apresentados os erros na norma $L_{1}$, a ordem de convergência observada $q$ e a constante do erro $C_{E}=\|E\|_{1} / \delta x^{q}$ dada pela Eq. (3.45) (ver Roache (1994)). Vê-se por essa tabela que a ordem do método global, equipado com o esquema TDPUS-C3, encontra-se acima de dois nas últimas cinco malhas, e que os valores da constante $C_{E}$ indicam que o método numérico (praticamente) atinge o intervalo assintótico de convergência. 
Tabela 5.5: Teste 2: teste de convergência obtido com TDPUS-C3. $t=1$ e $C F L=0.5$.

\begin{tabular}{cccc}
\hline \hline \multicolumn{4}{c}{ TDPUS-C3 } \\
\hline Malha & Erro em $L_{1}$ & Ordem $q$ & $C_{E}$ \\
\hline 20 & $4.26 E-01$ & - & - \\
40 & $1.06 E-01$ & 2.01 & 43.27 \\
80 & $3.29 E-02$ & 1.68 & 16.64 \\
160 & $9.60 E-03$ & 1.78 & 23.12 \\
320 & $2.26 E-03$ & 2.09 & 89.84 \\
640 & $5.12 E-04$ & 2.14 & 119.01 \\
1280 & $1.18 E-04$ & 2.12 & 101.68 \\
2560 & $2.80 E-05$ & 2.08 & 81.31 \\
5120 & $6.79 E-06$ & 2.04 & 61.28 \\
\hline \hline
\end{tabular}

\subsubsection{Equação não linear de Burgers}

Para simular fenômenos não lineares envolvendo advecção, nesta seção considera-se a equação de Burgers sem coeficiente de difusão dada pela Eq. (3.1) com fluxos (convexos) $f(u)=g(u)=0.5 u^{2}$. A equação de Burgers é um teste muito interessante, pois, mesmo no casos de condições iniciais suaves, ela é propensa à formação de descontinuidades; há casos em que existem soluções exatas desse problema (ver, por exemplo, Platzman (1964) e Debnath (1989)). Três casos teste, com condições de contorno periódicas, são simulados fazendo o uso dos esquemas TDPUS-C3, ARORA-ROE, CUBISTA, ADBQUICKEST e TOPUS: o (Teste 3) onde se investiga a variação total do esquema TDPUS-C3 no caso 1D; o (Teste 4) onde se investiga a solução antes e depois de um choque e comparações no caso 1D; o (Teste 5) onde se estimam os erros, a ordem de convergência observada e comparações no caso $2 \mathrm{D}$.

Para a simulação do Teste $\mathbf{3}$, foram considerados a condição inicial $u_{1}(x, 0)=\sin x$ em $[-1,1]$, quatro malhas $N=20,40,80$ e 160 células computacionais, tempo final de simulação $t=0.3 \mathrm{e}$ $C F L=0.5$. Os resultados estão apresentados na Fig. 5.4, onde pode-se observar que o esquema TDPUS-C3 satisfaz a condição dada pela Eq. (2.14).

Apresenta-se no Teste 4 soluções numéricas antes e depois do choque. Para tanto, utilizaram-se a condição inicial $u_{1}(x, 0)=\frac{1}{2}+\sin (\pi x)$ para $[-1,1]$, dois tempos finais de simulação, $t \approx 0.33$ (antes do choque ) e $t \approx 1.5$ (depois do choque), malha computacional de $N=80$ células e $C F L=$ 0.5. A solução (quase) analítica é obtida pelo algoritmo proposto na seção 4 da referência Teng (2010). Nas Figs. 5.5(a) e 5.5(b) estão apresentadas as soluções numéricas antes e depois do choque, respectivamente, calculadas com o esquema TDPUS-C3 mais a solução analítica. Observa-se por essa figura que o esquema TDPUS-C3 resolve bem a onda suave (antes do choque) e captura o choque com pequenas suavizações nos extremos; esses amortecimentos são causados pela inclusão na formulação do esquema FOU. A Fig. 5.6 mostra uma comparação entre as várias soluções numéricas obtidas com os esquemas upwind já mencionados anteriormente. Por essa figura, observa-se que os esquemas TDPUS-C3, CUBISTA e TOPUS forneceram, praticamente, a mesma solução; os esquemas ARORA-ROE e ADBQUICKEST mostraram-se inferiores.

Alguns dados de simulações para o Teste 5 são apresentados a seguir usando os vários esquemas de convecção, onde mostram-se os erros, a ordem de convergência e comparações no caso $2 \mathrm{D}$ da equação 


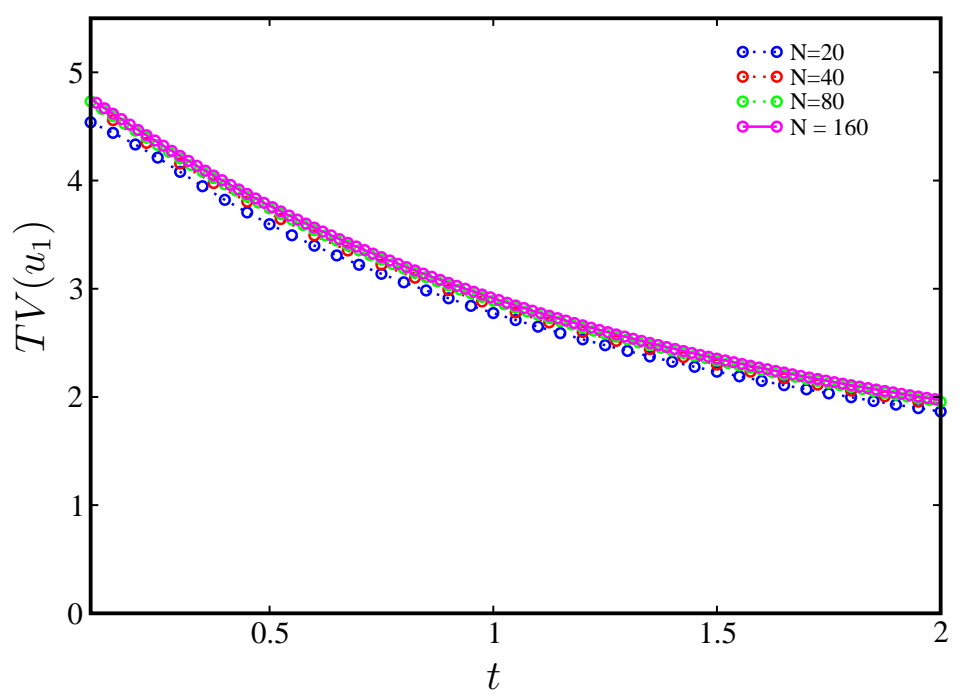

Figura 5.4: Teste 3: análise TVD para o esquema TDPUS-C3. $C F L=0.5$.

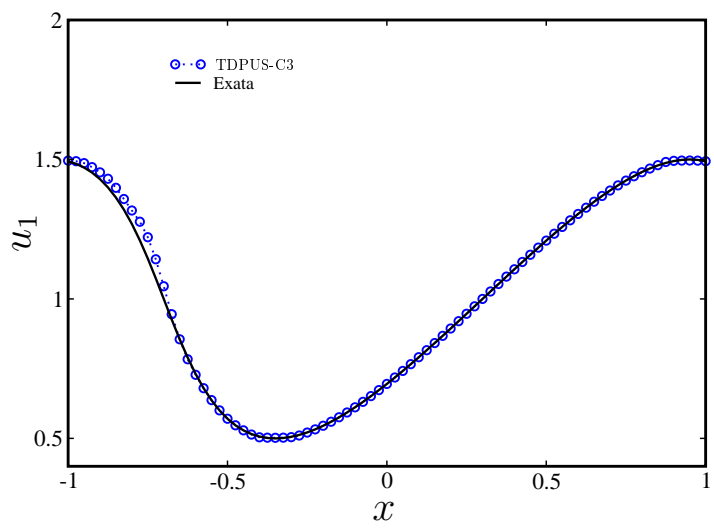

(a)

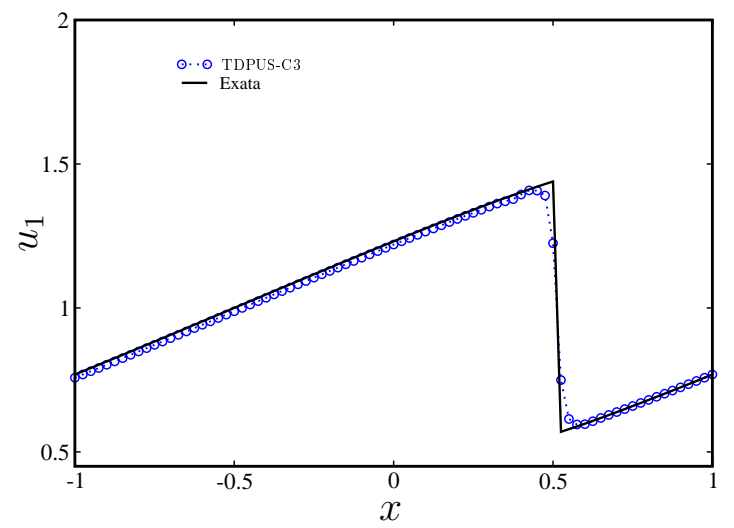

(b)

Figura 5.5: Teste 4: soluções numéricas da equação invíscida de Burgers obtidas com o TDPUS-C3 antes (a) e depois (b) da formação do choque. $N=80$ e $C F L=0.5$. 


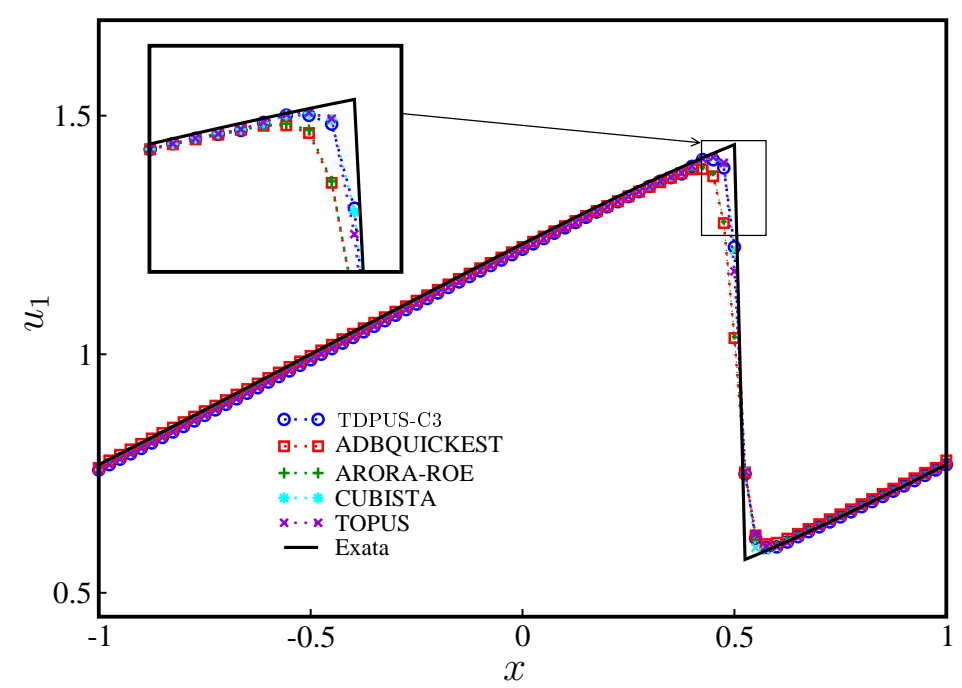

Figura 5.6: Teste 4: soluções numéricas da equação inviscida de Burgers depois do choque obtidas com TDPUS-C3, ADBQUICKEST, ARORA-ROE, CUBISTA e TOPUS. $t \approx 1.5, C F L=0.5 \mathrm{e}$ $N=80$.

não linear de Burgers. Para tanto, a condição inicial considerada é $\left.u(x, y, 0)=\frac{1}{2}+\sin (\pi(x+y) / 2)\right)$, em $[0,4] \times[0,4]$ e condições de contorno periódicas foram aplicadas. A solução analítica (na forma implícita) do problema é dada por $u(x, y, t)=\frac{1}{2}+\sin [\pi((x+y) / 2-u t)]$. Nas simulações foram empregados número de Courant $C F L=0.4$ e tempo final de simulação $t \approx 0.5 / \pi$ (antes da formação da onda de choque que ocorre para $t \approx 1.5 / \pi$ ). As Tabs. 5.6 e 5.7 apresentam os erros e as ordens de convergência observadas nas normas $L_{1}$ e $L_{\infty}$, respectivamente. Nota-se claramente pela Tab. 5.6 que todos os esquemas implementados forneceram ordens superiores a dois. Da Tab. 5.7 verifica-se que todos os esquemas superaram primeira ordem de precisão local nas malhas mais finas. As Tabs. 5.8 e 5.9 mostram os erros e as ordens de convergência observadas nas normas $L_{1}$ e $L_{\infty}$, respectivamente para os esquemas TDPUS-C3, EPUS, SDPUS-C1 e TOPUS. Vê-se claramente que as ordens de convergência são muito próximas.

Tabela 5.6: Teste 5: teste de convergência para a equação de Burgers 2D com TDPUS-C3, ADBQUICKEST, ARORA-ROE, CUBISTA e TOPUS na norma $L_{1} . t \approx 0.5 / \pi$ e $C F L=0.4$.

\begin{tabular}{ccccccccccc}
\hline \hline & \multicolumn{2}{c}{ TDPUS-C3 } & \multicolumn{2}{c}{ ADBQUICKEST } & \multicolumn{2}{c}{ ARORA-ROE } & \multicolumn{2}{c}{ CUBISTA } & & \multirow{2}{*}{ TOPUS } \\
\hline Malha & $L_{1}$ & $q$ & $L_{1}$ & $q$ & $L_{1}$ & $q$ & $L_{1}$ & $q$ & $L_{1}$ & $q$ \\
\hline $20 \times 20$ & $1.87 E-01$ & - & $1.87 E-01$ & - & $1.87 E-01$ & - & $1.87 E-01$ & - & $1.87 E-01$ & - \\
$40 \times 40$ & $4.27 E-02$ & 2.13 & $4.26 E-02$ & 2.13 & $4.29 E-02$ & 2.13 & $4.25 E-02$ & 2.14 & $4.28 E-02$ & 2.13 \\
$80 \times 80$ & $9.37 E-03$ & 2.19 & $9.77 E-03$ & 2.13 & $9.76 E-03$ & 2.13 & $9.30 E-03$ & 2.19 & $9.27 E-03$ & 2.21 \\
$160 \times 160$ & $2.02 E-03$ & 2.21 & $2.15 E-03$ & 2.18 & $2.15 E-03$ & 2.18 & $2.04 E-03$ & 2.19 & $2.07 E-03$ & 2.17 \\
$320 \times 320$ & $4.58 E-04$ & 2.14 & $4.55 E-04$ & 2.24 & $4.55 E-04$ & 2.24 & $4.68 E-04$ & 2.12 & $4.73 E-04$ & 2.13 \\
\hline \hline
\end{tabular}


Tabela 5.7: Teste 5: teste de convergência para a equação de Burgers 2D com TDPUS-C3, ADBQUICKEST, ARORA-ROE, CUBISTA e TOPUS na norma $L_{\infty} . t \approx 0.5 / \pi$ e $C F L=0.4$.

\begin{tabular}{|c|c|c|c|c|c|c|c|c|c|c|}
\hline & \multicolumn{2}{|c|}{ TDPUS- $C 3$} & \multicolumn{2}{|c|}{ ADBQUICKEST } & \multicolumn{2}{|c|}{ ARORA-ROE } & \multicolumn{2}{|c|}{ CUBISTA } & \multicolumn{2}{|c|}{ TOPUS } \\
\hline Malha & $L_{\infty}$ & $q$ & $L_{\infty}$ & $q$ & $L_{\infty}$ & $q$ & $L_{\infty}$ & $q$ & $L_{\infty}$ & $q$ \\
\hline $20 \times 20$ & $6.51 E-01$ & - & $6.12 E-01$ & - & $6.09 E-01$ & - & $6.81 E-01$ & - & $6.95 E-01$ & - \\
\hline $40 \times 40$ & $3.29 E-01$ & 0.98 & $3.45 E-01$ & 0.83 & $3.45 E-01$ & 0.82 & $3.40 E-01$ & 1.00 & $3.48 E-01$ & 1.00 \\
\hline $80 \times 80$ & $1.69 E-01$ & 0.96 & $1.79 E-01$ & 0.95 & $1.78 E-01$ & 0.95 & $1.69 E-01$ & 1.01 & $1.70 E-01$ & 1.03 \\
\hline $160 \times 160$ & $7.79 E-02$ & 1.12 & $8.68 E-02$ & 1.04 & $8.68 E-02$ & 1.04 & $7.74 E-02$ & 1.13 & $7.69 E-02$ & 1.15 \\
\hline $320 \times 320$ & $3.39 E-02$ & 1.20 & $4.01 E-02$ & 1.11 & $4.01 E-02$ & 1.11 & $3.53 E-02$ & 1.14 & $3.55 E-02$ & 1.12 \\
\hline
\end{tabular}

Tabela 5.8: Teste 5: teste de convergência para a equação de Burgers 2D com TDPUS-C3, EPUS, SDPUS-C1 e TOPUS na norma $L_{1} . t \approx 0.5 / \pi$ e $C F L=0.4$.

\begin{tabular}{ccccccccc}
\hline \hline & \multicolumn{2}{c}{ TDPUS-C3 } & \multicolumn{2}{c}{ EPUS } & \multicolumn{2}{c}{ SDPUS-C1 } & \multicolumn{2}{c}{ TOPUS } \\
\hline Malha & $L_{\infty}$ & $q$ & $L_{\infty}$ & $q$ & $L_{\infty}$ & $q$ & $L_{\infty}$ & $q$ \\
\hline $20 \times 20$ & $1.87 E-01$ & - & $4.61 E-02$ & - & $4.70 E-02$ & - & $1.87 E-01$ & - \\
$40 \times 40$ & $4.27 E-02$ & 2.13 & $1.05 E-02$ & 2.13 & $1.08 E-02$ & 2.13 & $4.28 E-02$ & 2.13 \\
$80 \times 80$ & $9.37 E-03$ & 2.19 & $2.39 E-03$ & 2.14 & $2.35 E-03$ & 2.19 & $9.27 E-03$ & 2.21 \\
$160 \times 160$ & $2.02 E-03$ & 2.21 & $5.11 E-04$ & 2.23 & $5.10 E-04$ & 2.20 & $2.07 E-03$ & 2.17 \\
$320 \times 320$ & $4.58 E-04$ & 2.14 & $1.16 E-04$ & 2.14 & $1.16 E-04$ & 2.14 & $4.73 E-04$ & 2.13 \\
\hline \hline
\end{tabular}

Tabela 5.9: Teste 5: teste de convergência para a equação de Burgers 2D com TDPUS-C3, EPUS, SDPUS-C1 e TOPUS na norma $L_{\infty} . t \approx 0.5 / \pi$ e $C F L=0.4$.

\begin{tabular}{ccccccccc}
\hline \hline & \multirow{2}{*}{ TDPUS-C3 } & \multicolumn{2}{c}{ EPUS } & \multicolumn{2}{c}{ SDPUS-C1 } & \multicolumn{2}{c}{ TOPUS } \\
\hline Malha & $L_{\infty}$ & $q$ & $L_{\infty}$ & $q$ & $L_{\infty}$ & $q$ & $L_{\infty}$ & $q$ \\
\hline $20 \times 20$ & $6.51 E-01$ & - & $6.96 E-01$ & - & $6.57 E-01$ & - & $6.95 E-01$ & - \\
$40 \times 40$ & $3.29 E-01$ & 0.98 & $3.57 E-01$ & 0.96 & $3.304 E-01$ & 0.99 & $3.48 E-01$ & 1.00 \\
$80 \times 80$ & $1.69 E-01$ & 0.96 & $1.67 E-01$ & 1.09 & $1.69 E-01$ & 0.97 & $1.70 E-01$ & 1.03 \\
$160 \times 160$ & $7.79 E-02$ & 1.12 & $7.80 E-02$ & 1.10 & $7.73 E-02$ & 1.13 & $7.69 E-02$ & 1.15 \\
$320 \times 320$ & $3.39 E-02$ & 1.20 & $3.37 E-02$ & 1.21 & $3.39 E-02$ & 1.19 & $3.55 E-02$ & 1.12 \\
\hline \hline
\end{tabular}


Na Fig. 5.7 estão apresentados, no tempo $t \approx 0.5 / \pi$ e ao longo do plano $x=y$, resultados numéricos na malha $160 \times 160$ com o uso dos vários esquemas estudados e a solução analítica. Por essa figura, observa-se que os esquemas TDPUS-C3, CUBISTA e TOPUS forneceram, praticamente, a mesma solução; os esquemas ARORA-ROE e ADBQUICKEST mostraram-se inferiores. A Fig. 5.8 mostra as comparações das soluções numéricas ao longo do plano $x=y$ na malha $160 \times 160$ dos esquemas TDPUS-C3, EPUS, SDPUS-C1 e TOPUS. Vê-se claramente que, com condição inicial suave, o SDPUS-C1 e TOPUS superam ligeiramente o esquema TDPUS-C3 nos picos. O EPUS foi inferior nessa região. Nos vales, o TDPUS-C3 apresentou resultados mais próximo da solução exata.

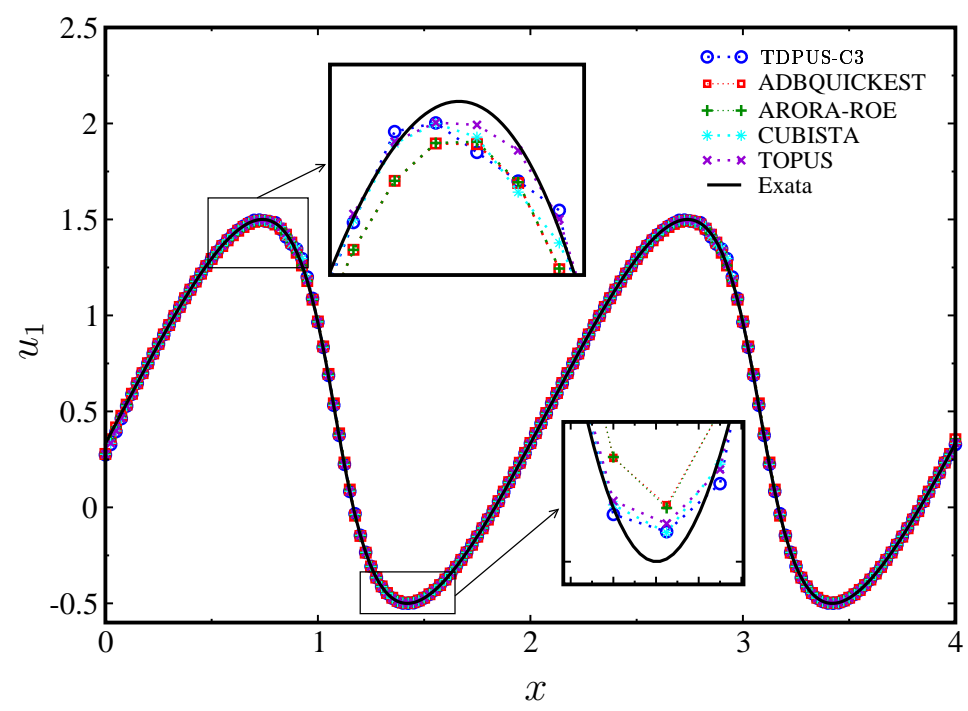

Figura 5.7: Teste 5: soluções numéricas ao longo do plano $x=y$ da equação de Burgers 2D com TDPUS-C3, ADBQUICKEST, ARORA-ROE, CUBISTA e TOPUS. $t=0.5 / \pi, 160 \times 160$ células computacionais e $C F L=0.4$ 


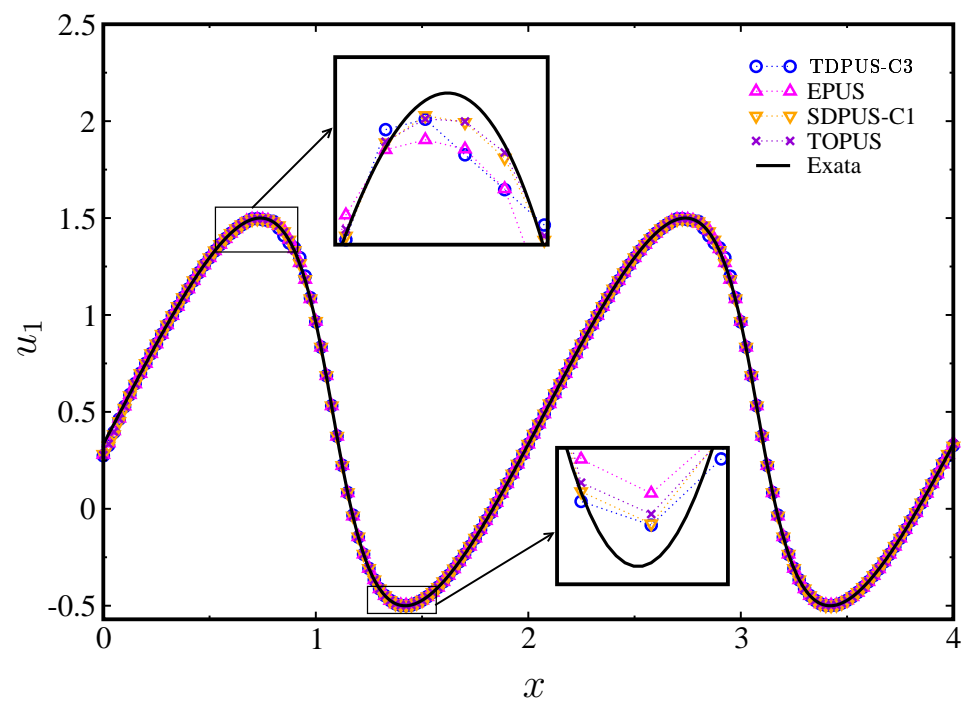

Figura 5.8: Teste 5: soluções numéricas ao longo do plano $x=y$ da equação de Burgers $2 \mathrm{D}$ com TDPUS-C3, EPUS, SDPUS-C1 e TOPUS. $t=0.5 / \pi, 160 \times 160$ células computacionais e $C F L=0.4$

\subsubsection{Equação não linear de Buckley-Leverett}

Considera-se agora o Teste 6, em que pretende-se simular a lei de conservação não convexa de Buckley-Leverett 1D dada pela Eq. (3.1) com fluxo (não convexo) $f(u)=\frac{u_{1}^{2}}{4 u_{1}^{2}+\left(1-u_{1}\right)^{2}}$. Essa lei modela o escoamento de um fluido bifásico em um meio poroso (LeVeque, 1992), cuja solução de entropia pode apresentar choques, ondas de rarefação e descontinuidade de contato. Para a simulação deste problema, toma-se a condição inicial

$$
u_{1}(x, 0)= \begin{cases}1, & -\frac{1}{2} \leq x \leq 0 \\ 0, & \text { no caso contrario }\end{cases}
$$

e condições periódicas no contorno do domínio $[-1,1]$. Foram considerados uma malha de $N=200$ células, número de Courant $C F L=0.4$ e tempo final de simulação $t=0.4$. A solução de referência para esse problema é tomada como a solução numérica na malha $N=2000$ células computacionais usando o limitador de fluxo não linear de van Leer (1974); limitador esse frequentemente empregado em leis de conservação não convexa e em problemas envolvendo mistura de ondas de choque, de rarefação e descontinuidade de contato. É importante comentar neste ponto que alguns esquemas de alta resolução não conseguem soluções convergentes para a solução correta de entropia (LeVeque, 1992). Na Fig. 5.9, e também em suas ampliações Figs. 5.10 (a) e 5.10 (b), estão representadas as soluções numéricas calculadas com o esquema upwind TDPUS-C3 mais os convencionais CUBISTA, TOPUS, ARORA-ROE e ADBQUICKEST. No global, por essas figuras, pode-se fazer uma classificação dos esquemas upwind implementados, com respeito a melhor aproximação, da seguinte maneira: TDPUS-C3, CUBISTA, TOPUS, ADBQUICKEST e ARORA-ROE. Em particular, na Fig. 5.10(b) observa-se que os esquemas CUBISTA e TDPUS-C3 fornecem resultados semelhantes e resolvem bem a descontinuidade de contato. 


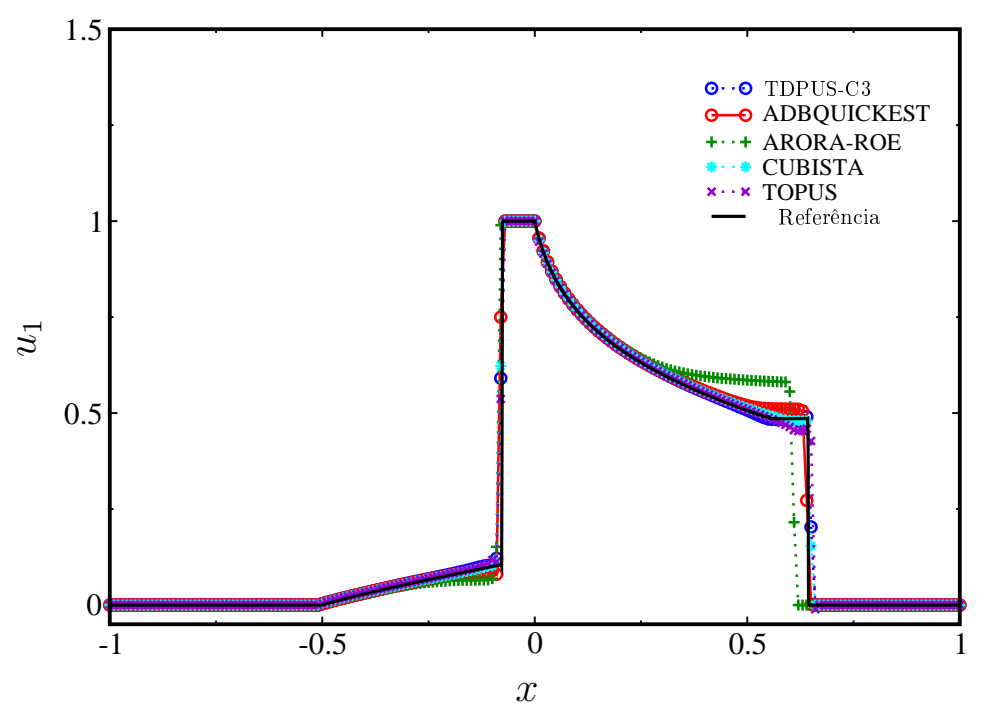

Figura 5.9: Teste 6: soluções numéricas para a equação não linear de Buckley-Leverett. $t=0.4$, $C F L=0.4$ e $N=200$.

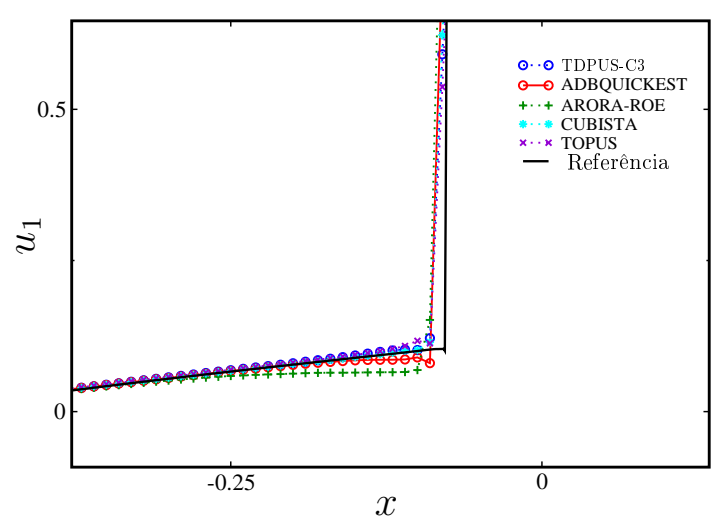

(a)

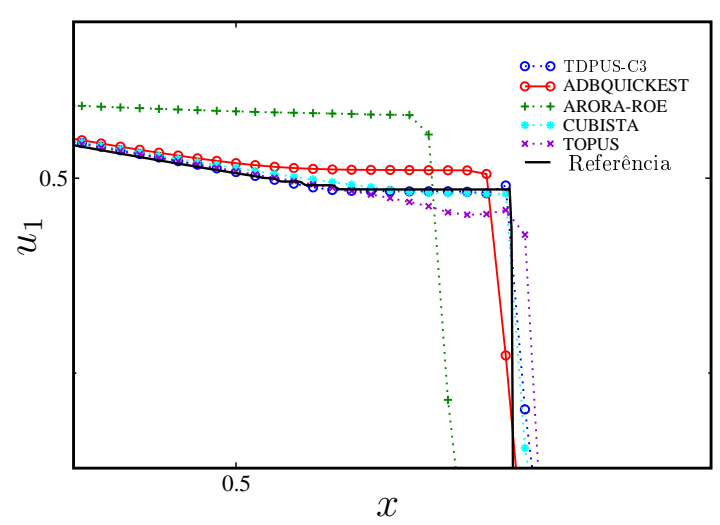

(b)

Figura 5.10: Teste 6: soluções numéricas da equação de Buckley-Leverett. Região do choque $x \approx$ -0.077 e da descontinuidade de contato $x \approx 0.64$. $t=0.4, N=200$ e $C F L=0.4$. 


\subsection{Sistemas 1D e 2D de leis de conservação}

Nesta seção são apresentados resultados numéricos com o esquema TDPUS-C3 para sistemas não lineares complexos de leis de conservação. Em particular, são mostrados resultados para equações complexas de águas rasas e Euler, ambas com ou sem termo fonte, e equações da MHD. Apresentam-se também comparações dos resultados obtidos com o TDPUS-C3 e os obtidos com os esquemas ADBQUICKEST, ARORA-ROE, CUBISTA, SUPERBEE, MINMOD, MC, TOPUS, SDPUS-C1 e EPUS.

\subsubsection{Equações de águas rasas}

Apresenta-se nesta seção resultados para as equações de águas rasas nos casos homogêneo 1D (Teste 7), com termo fonte 1D (Teste 8) e homogêneo 2D (Teste 9). Essas equações são dadas por (3.1) com variáveis de estado $\left(h, h u_{1}, h u_{2}\right)^{T}$ e funções fluxo $f(u)=\left(h u_{1}, h u_{1}^{2}+\frac{1}{2} g h^{2}, h u_{1} u_{2}\right)^{T}$, e $g(u)=\left(h u_{2}, h u_{1} u_{2}, h u_{2}^{2}+\frac{1}{2} g h^{2}\right)^{T}$. A quantidade $h$ denota a profundidade da água, $u_{1}$ e $u_{2}$ são os componentes do vetor velocidade $u ; h u_{1}$ e $h u_{2}$ são as vazões e $g=9.81 \mathrm{~m}^{2} / \mathrm{s}$ é a constante gravitacional. Para os casos Teste 7 e Teste 9, os termos fontes são $S(u)=(0,0)^{T}$ e $S(u)=(0,0,0)^{T}$, respectivamente, e para o Teste 8 o termo fonte é $S(u)=\left(0,-g h B^{\prime}\right)^{T}$, com $B^{\prime}$ a derivada da função que descreve o fundo do canal $B=B(x)$ (topografia), ver Fig. 5.11. As velocidades absolutas das ondas nestas equações para o caso 1D são os autovalores da matriz Jacobiana (Eq. (3.1)) $\lambda_{1,2}=u_{1} \pm c$, sendo $c=\sqrt{g h}$ a celeridade. O adimensional que caracteriza um problema modelado por essas equações é o número de Froude, $F r=u_{1} / c$ : $\operatorname{subcrítico~}(F r<1)$, supercrítico $(F r>1)$ ou crítico $(F r=1)$.

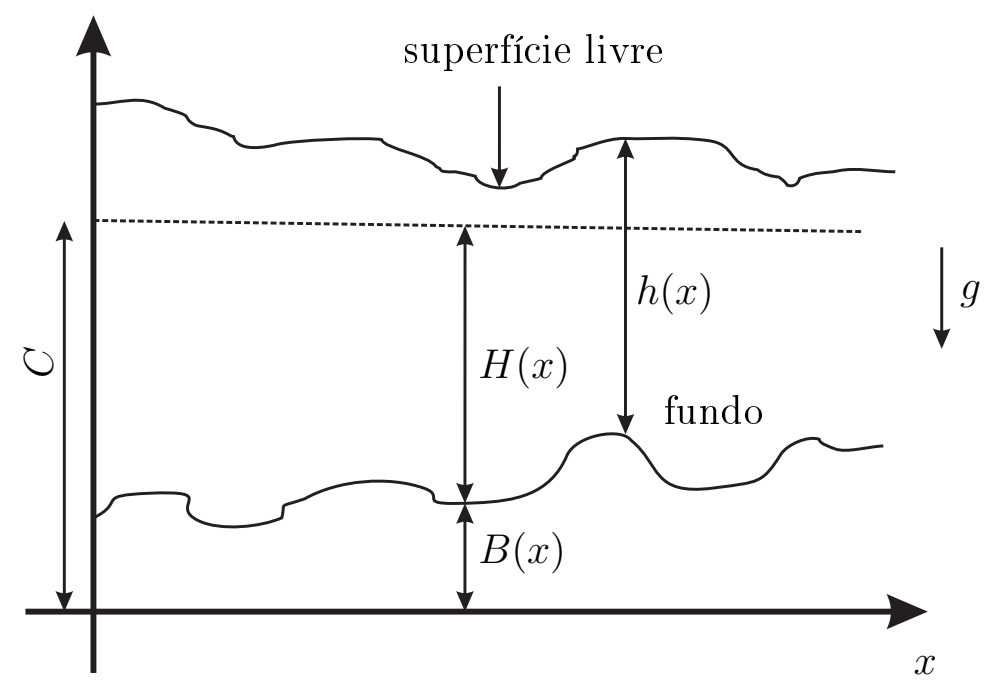

Figura 5.11: Ilustração esquemática de um escoamento com superfície livre sobre a influência da gravidade.

O Teste 7, apresentado a partir de agora, constitui um problema interessante de ruptura de barragem, cuja solução pode ser obtida via um problema de Riemann num canal contendo uma barragem que separa o fluido em dois níveis diferentes de água. Para a simulação desse problema foram utilizadas 
as condições inicias (ver Simões et al. (2011))

$$
h(x, 0)=\left\{\begin{array}{ll}
8, & x \leq 500, \\
4, & x>500,
\end{array} \quad u_{1}(x, 0)=0,\right.
$$

e condições periódicas no contorno do domínio computacional $[0,1000]$. Os seguintes dados foram empregados na simulação: malha computacional de $N=400$ células, número de Courant $C F L=0.91$ e tempos finais de simulação $t=10,20,30,40$ e 50. A solução exata desse problema pode ser obtida usando-se a fórmula de Stoker (1956) (ver detalhes da derivação da solução em Simões et al. (2011)). Os resultados numéricos com o esquema TDPUS-C3 em vários tempos de simulação estão descritos na Fig. 5.12, onde pode-se observar que, no geral, há uma ótima concordância entre esses dados numéricos e a solução exata, mostrando, em particular, que a onda de rarefação e o choque são simulados com sucesso. Observa-se também que para tempos pequenos $(t=10)$ de simulação o esquema é propenso à formação de pequenas oscilações para $400<x<600$, mas essas instabilidades são amortecidas com o passar do tempo.

A Fig. 5.13 mostra no tempo de simulação $t=10$, comparações das soluções numéricas obtidas com os vários esquemas, incluindo o TDPUS-C3, onde pode ser visto que todos os esquemas forneceram resultados bastante semelhantes.

O Teste 8, descrito a seguir, representa um problema interessante de águas rasas com termo fonte para descrever a topografia do fundo de um canal. Nesse problema pode ser observado um escoamento subcrítico (antes da elevação do fundo), crítico (no ponto máximo da elevação $-x=10$ ) e supercrítico (antes do choque - $x \approx 12$ ); no choque ele volta a ser crítico e depois do choque subcrítico. Objetiva-se estudar o desempenho do esquema TDPUS-C3 em tempo longo de simulação e comparar a solução numérica com as soluções numéricas dos demais esquemas estudados. Esse teste tem sido considerado benchmark para investigar a habilidade de um esquema numérico em capturar choques em problemas de águas rasas (ver, por exemplo Xing e Shu (2005)). Para a simulação do problema, consideram-se as mesmas condições empregadas em Bouchut (2000) e Goutal e Maurel (1997), para $x \in[0,25]$, a saber: (i) condições iniciais $h=0.33$ e $h u=0.18$; e (ii) condições de contorno $h(x=25)=0.33$, $h u(x=0)=0.18$ e os valores para $h(x=0)$ e $h u(x=25)$ são obtidos de extrapolação de primeira ordem. Os seguintes dados foram utilizados: malha computacional de $N=200$ células, a número de Courant $C F L=0.9$ e tempo final de simulação $t=200$. A solução de referência para o nível de água considerada para este teste é obtida usando o esquema convectivo MINMOD (Roe, 1986) com $N=2000$ células computacionais; para a vazão, a solução de referência é a solução exata $h u=0.18$. A topografia do fundo contemplada no termo fonte $S$ da equação de águas rasas é definida por

$$
B(x)=\left\{\begin{array}{l}
\left.0.2-0.05(x-10)^{2}\right), \quad 8 \leq x \leq 12 \\
0, \quad \text { caso contrário. }
\end{array}\right.
$$

As soluções (em estado estacionário) de referência e numéricas para o nível d'água e vazão estão representadas nas Figs. 5.14 (a) e 5.14 (b), respectivamente. Observa-se na Fig. 5.14 (a) que todas as soluções numéricas são de boa qualidade, quando comparadas com a solução numérica de referência 


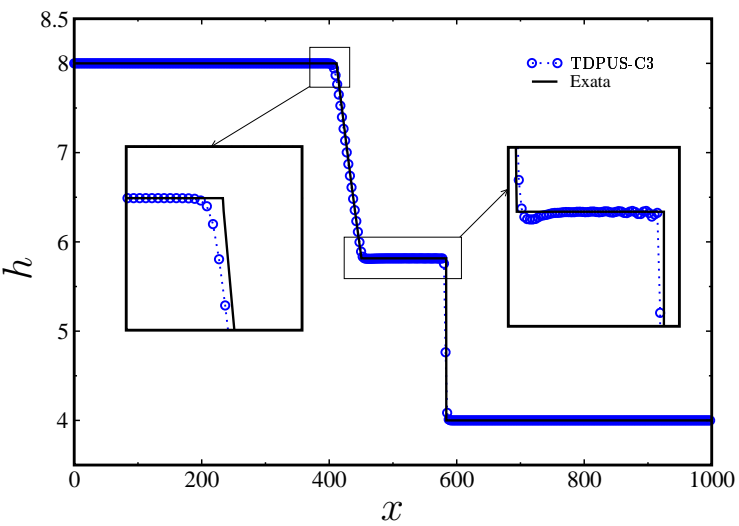

$t=10$

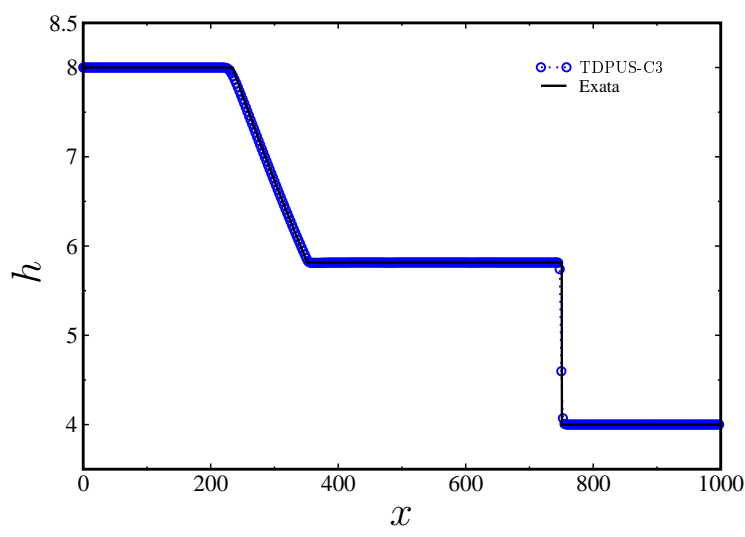

$t=30$

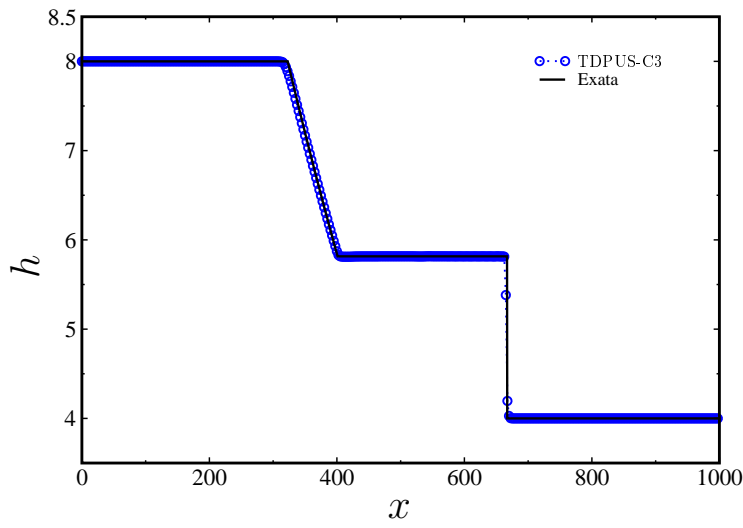

$t=20$

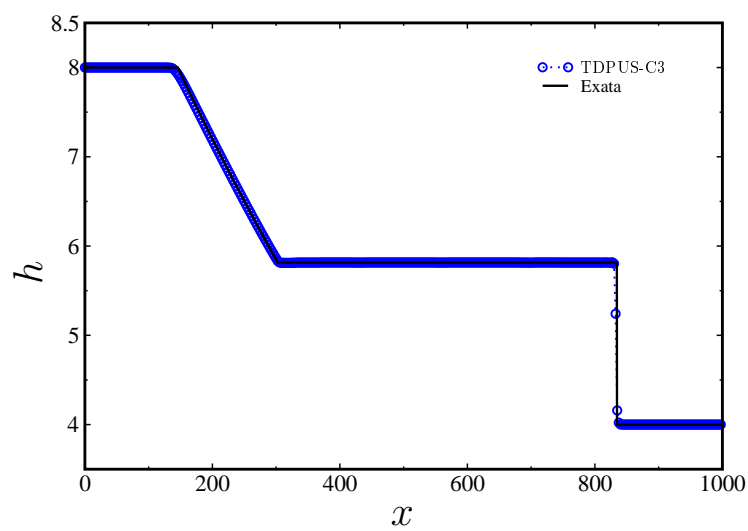

$t=40$

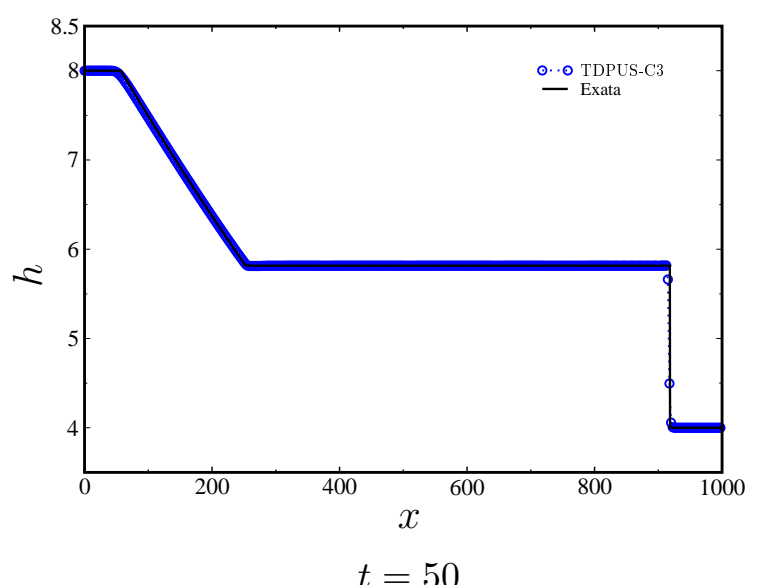

Figura 5.12: Teste 7: soluções numéricas com TDPUS-C3 e a solução exata para o nível da água $h$ no problema de ruptura da barragem. $N=400$ e $C F L=0.91$.

na malha $N=2000$. Na Fig. 5.14 (b), os resultados numéricos com os vários esquemas apresentam problemas na captura correta da vazão; em particular, o TDPUS-C3 superestimou (ligeiramente) a solução nas vizinhanças do choque. Vale observar que, como notado por muitos autores (ver, por 


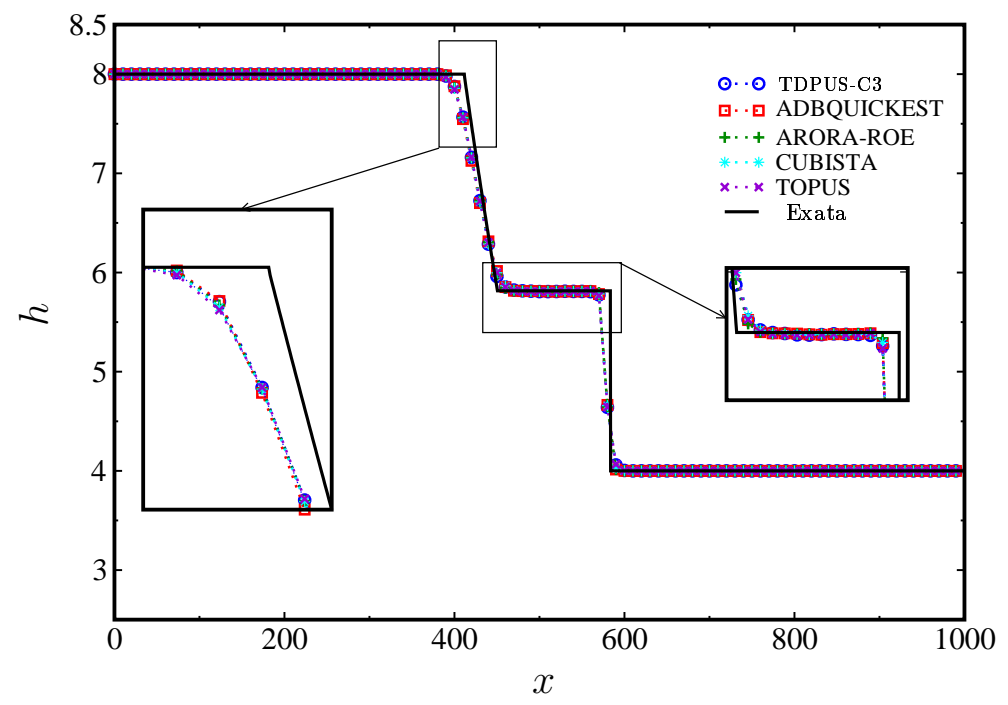

Figura 5.13: Teste 7: comparação entre as soluções numéricas para o nível da água $h$ com TDPUS-C3, ADBQUICKEST, ARORA-ROE, CUBISTA, TOPUS e a solução exata. $t=10$, $N=100$ e $C F L=0.91$.

exemplo, Xing e Shu (2005) e Kun (2002)), a captura correta da vazão é muito mais difícil de se obter que a do nível da água. Para quantificação, a diferencia entre as soluções obtidas com o esquema TDPUS-C3 e SUPERBEE (um esquema útil da literatura) é de $\approx 10^{-5}$.

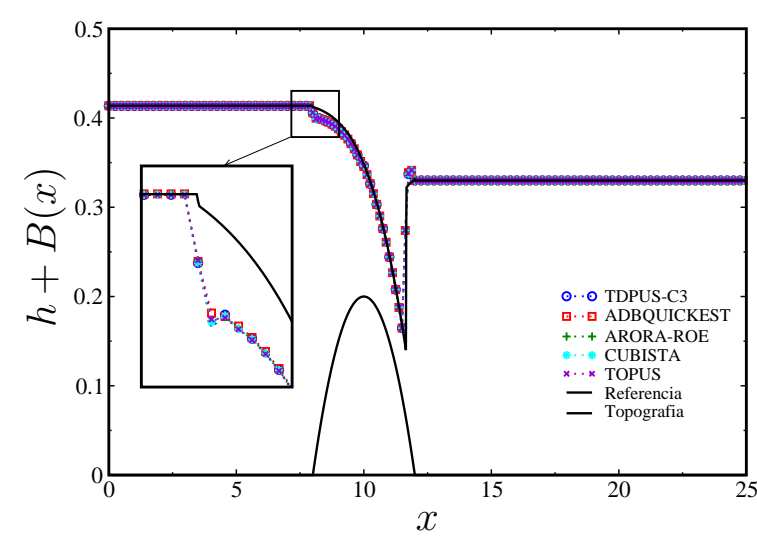

(a)

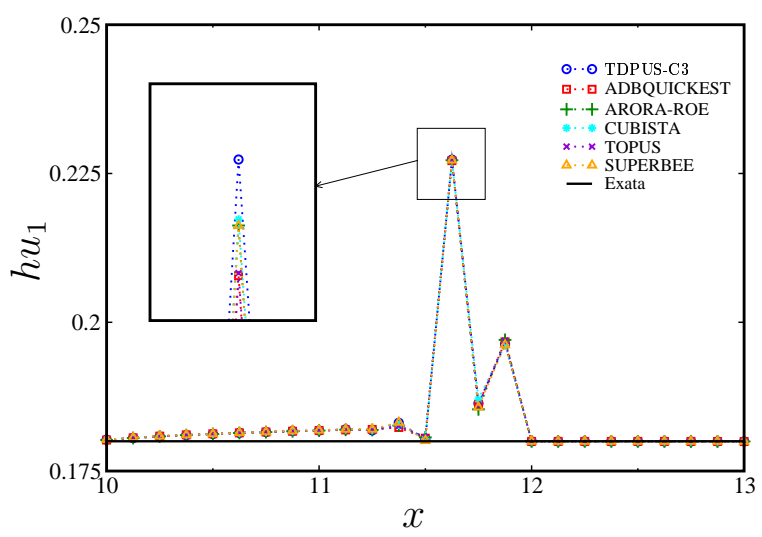

(b)

Figura 5.14: Teste 8: nível da água $h+B(x)$ (a) e a vazão $h u$ (b). $t=200, N=200$ e $C F L=0.9$.

O Teste 9, apresentado na sequência, finaliza os testes para equações de águas rasas; ele constitui a ruptura de uma barragem circular. Neste problema, uma onda de choque é propagada pela liberação de uma coluna de água de altura inicial $h=0.5$. O objetivo deste teste é demonstrar a habilidade do esquema TDPUS-C3 em reproduzir ondas de choque e de rarefação nas equações de águas rasas. Os resultados obtidos para a altura $h=h(x, y, t)$ da água, com $y=0$ e $t=1.4$, são comparados com os resultados numéricos obtidos com os métodos (centrados) FORCE de Stecca et al. (2010) e 
Godunov-HLL de Toro (1999). Da mesma forma como foi feito no artigo de Stecca et al. (2010), o problema foi simulado no domínio $[-20,20] \times[-20,20]$ usando-se várias malhas $(100 \times 100,200 \times 200$ e $600 \times 600$ ), a número de Courant $C F L=0.45$, tempo final de simulação $t=1.4$ e com condição inicial dada por

$$
\left\{\begin{array}{l}
h(x, y, 0)=2.5, \quad x^{2}+y^{2} \leq R^{2} \\
h(x, y, 0)=0.5, \quad x^{2}+y^{2}>R^{2} \\
u(x, y, 0)=0, \quad \forall x, y \\
v(x, y, 0)=0, \quad \forall x, y
\end{array}\right.
$$

onde $R=2.5$ é o raio do tanque. As condições de contorno empregadas foram refletivas. A solução de referência adotada para esse problema é a mesma adotada por Stecca et al. (2010) (Godunov com termo de correção mais o limitador SUPERBEE, numa malha de $1000 \times 1000$ e número de Courant $C F L=0.9$ ). A Fig. 5.15 mostra os resultados numéricos, na malha grossa $100 \times 100$, obtidos com os métodos FORCE, GODUNOV- HLL e TDPUS-C3, onde pode-se observar que o esquema TDPUS-C3 reproduziu melhor a solução de referência. A Fig. 5.16 (figura principal (a) e ampliações (b) e (c)) apresenta as soluções numéricas com TDPUS-C3 em várias malhas, mostrando que ao se refinar a malha a solução numérica converge para uma solução bem próxima da de referência. Em resumo, pode-se dizer que o esquema TDPUS-C3 captura a física do problema e fornece o melhor resultado.

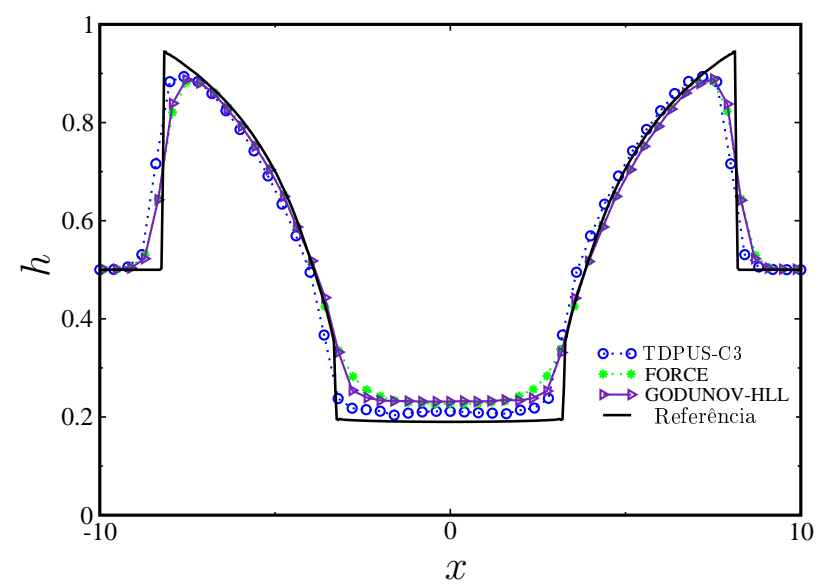

Figura 5.15: Teste 9: ruptura de uma barragem circular. Soluções numéricas para o nível da água $h$ ao longo do eixo $x(y=0)$, com TDPUS-C3, FORCE, GODUNOV-HLL. $t=1.4,100 \times 100$ células computacionais e $C F L=0.45$. 


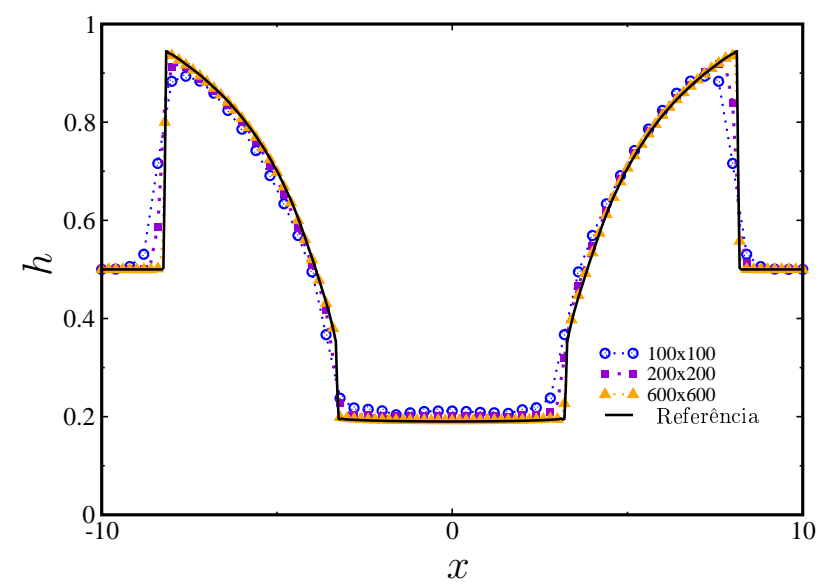

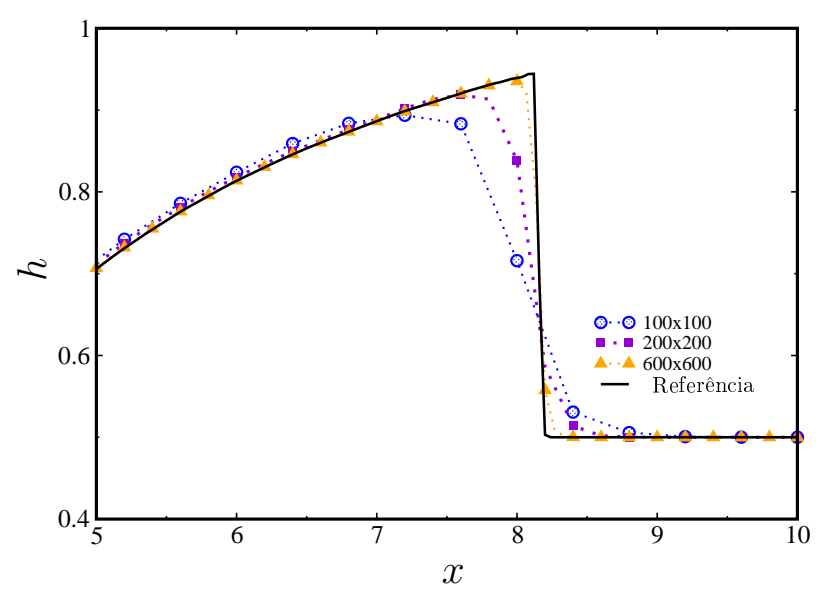

(b)

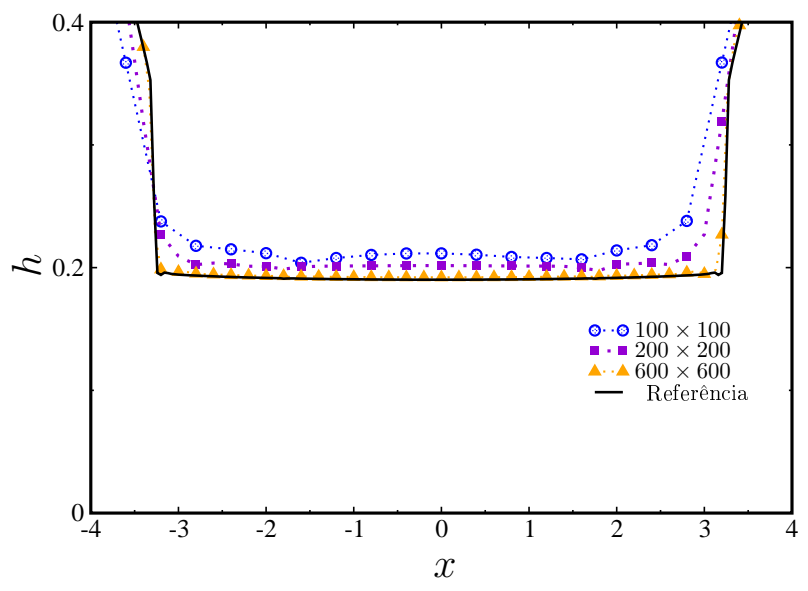

(c)

Figura 5.16: Teste 9: ruptura de uma barragem circular. Evolução espacial das soluções numéricas obtidas com TDPUS-C3 para o nível da água $h$ ao longo do eixo $x(y=0) . t=1.4$ e $C F L=0.45$. 


\subsubsection{Equações de Euler}

A partir de agora são apresentados resultados numéricos para problemas complexos modelados pelas equações de Euler nos casos homogêneo 1D (Teste 10 e Teste 11), homogêneo 2D (Teste 12 e Teste 13) e não homogêneo (Teste 14). As equações de Euler são dadas pela Eq. (3.1) com variáveis de estado $\left(\rho, \rho u_{1}, \rho u_{2}, E\right)^{T}$ e funções fluxo $f(u)=\left(\rho u_{1}, \rho u_{1}{ }^{2}+p, \rho u_{1} u_{2},(E+p) u_{1}\right)^{T}$ e $g(u)=\left(\rho u_{2}, \rho u_{1} u_{2}, \rho u_{2}{ }^{2}+p,,(E+p) u_{2}\right)^{T}$. A quantidade $\rho$ denota a densidade, $\rho u_{1}$ e $\rho u_{2}$ são as componentes do vetor quantidade de movimento. A energia total é definida por $E=\rho\left(\frac{1}{2}\left(u_{1}^{2}+u_{2}^{2}\right)\right)+e$, com $e=e(\rho, p)=\frac{p}{(\gamma-1) \rho}$. A quantidade $\gamma$ é a constante do calor específico. Para o caso Teste 14 o termo fonte escolhido é $S(u)=\left(0,0, \rho g, \rho u_{2} g\right)^{T}$. As outras quantidades foram definidas previamente.

O Teste 10, uma idealização da interação choque-turbulência (também conhecido como tubo de choque de Shu e Osher (1989)) consiste em uma onda de choque que se propaga em um campo em repouso, com pressão constante e perfil de densidade senoidal. Neste problema, o perfil da densidade possui duas partes distintas: a primeira tem a mesma frequência e amplitude do perfil dos dados iniciais, com ondas de compressão associadas a ondas de choque na forma clássica "N"; a segunda parte é de alta frequência e maior amplitude com ondas de choque mais próximas (ver Kamm et al. (2008)). O objetivo deste teste é mostrar que o esquema TDPUS-C3 é útil na simulação da interação de ondas de choque com turbulência. Os resultados apresentados a seguir são para a densidade $\rho$ no domínio $[-5,5]$. A simulação computacional foi executada em várias malhas $(N=125,250,500$ e 1000), número de Courant $C F L=0.5$, tempo final de simulação $t=1.8$, e a constante do calor específico $\gamma=1.4$. As condições iniciais adotadas são

$$
\left\{\begin{array}{lll}
x<0.4: \rho=3.857143 ; & u_{1}=2.629369 ; & p=10.33333 \\
x \geq 0.4: \rho=1+0.2 \sin (5 x) ; & u_{1}=0 ; & p=1 .
\end{array}\right.
$$

Extrapolação de ordem zero foi usada como condição de contorno. A solução de referência é calculada numa malha de $N=2000$ células com o método de Godunov com termo de correção e limitador SUPERBEE.

Na Fig. 5.17 está mostrada a comparação entre a solução de referência e os resultados com os esquemas TDPUS-C3, ADBQUICKEST, ARORA-ROE, CUBISTA e TOPUS, onde pode-se inferir que na região de alta frequência, o esquema TDPUS-C3 ficou em terceiro lugar como o melhor método. Fora dessa região de transição ampla (onda de entropia), o esquema TDPUS-C3 forneceu resultados similares aos dos outros esquemas, com exceção dos esquemas dependentes de $C F L$ (ADBQUICKEST e ARORA-ROE) que apresentaram uma melhor aproximação na região de entropia.

As Figs. 5.18 e 5.19 (essa última mostrando ampliações de algumas regiões da primeira) mostram a solução numérica para a densidade no problema de Shu-Osher com o esquema TDPUS-C3 em várias malhas. Vê-se claramente que, em todas as regiões do domínio, houve convergência do método numérico global para uma solução muito próxima da de referência.

O Teste 11, uma interação complexa de ondas de choque (também conhecido como problema ou tubo de choque de Woodward e Colella (1984)), é um problema desafiador para os métodos numéricos uma vez que há efeitos não lineares acentuados; estes sendo devido a interação de ondas de choque, rarefação e descontinuidades de contato (veja, por exemplo, Greenough e Rider (2004)). No tempo $t=0$ 


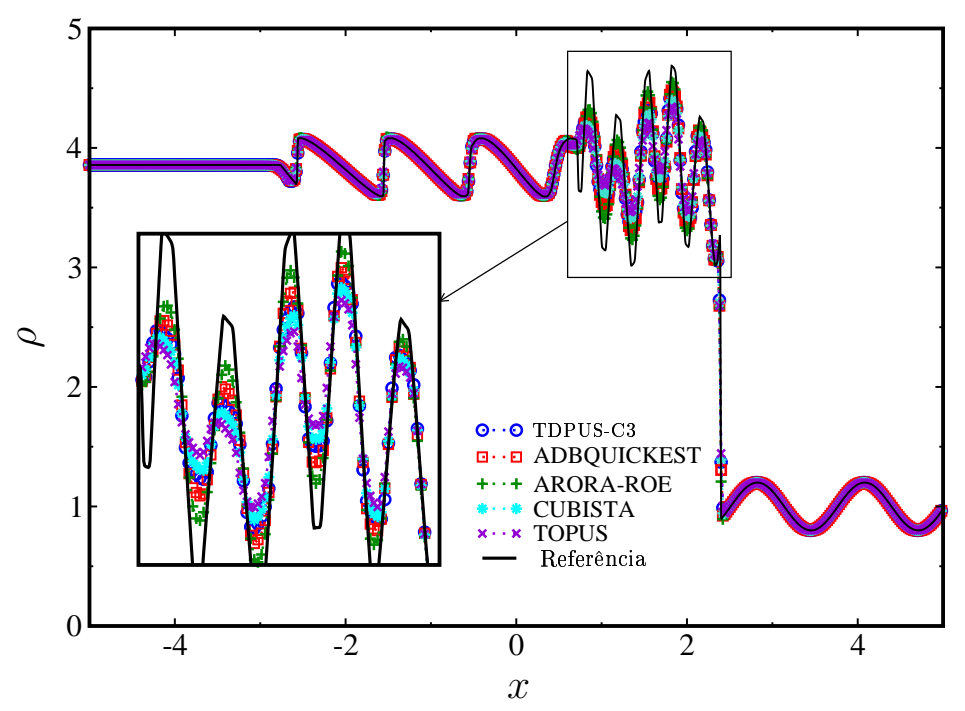

Figura 5.17: Teste 10: comparação das soluções numéricas da densidade $\rho$ para o problema de Shu-Osher com TDPUS-C3, ADBQUICKEST, ARORA-ROE, CUBISTA e TOPUS. $t=1.8, N=500$ e $C F L=0.5$.

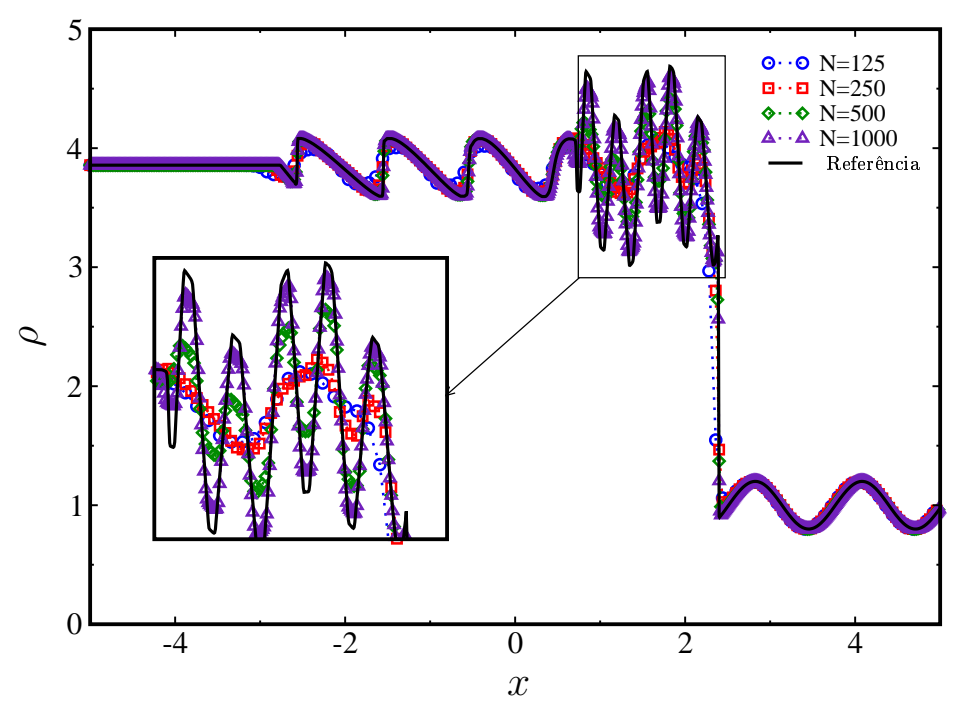

Figura 5.18: Teste 10: evolução espacial das soluções numéricas da densidade $\rho$ para o problema de Shu-Osher com TDPUS-C3, ADBQUICKEST, ARORA-ROE, CUBISTA e TOPUS. $t=1.8$, $N=500$ e $C F L=0.5$. 
$\mathrm{N}$-wave

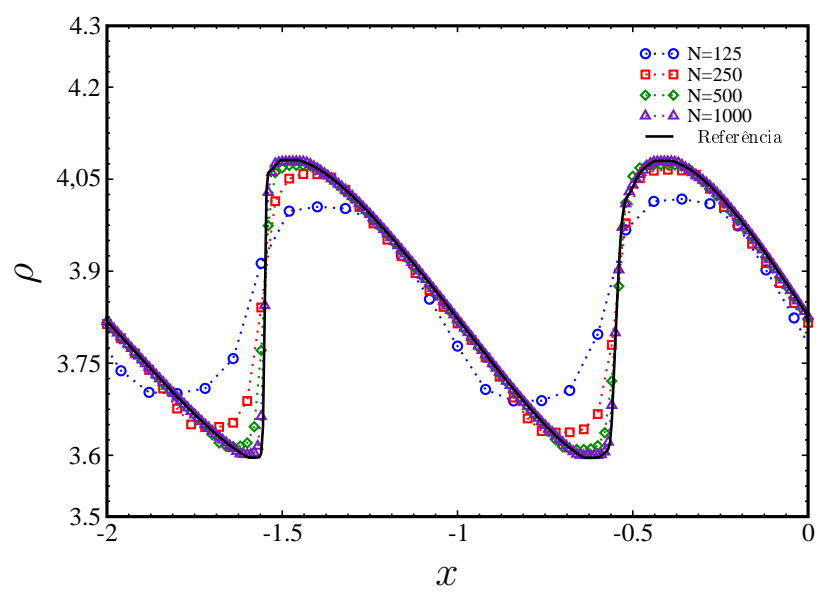

EW

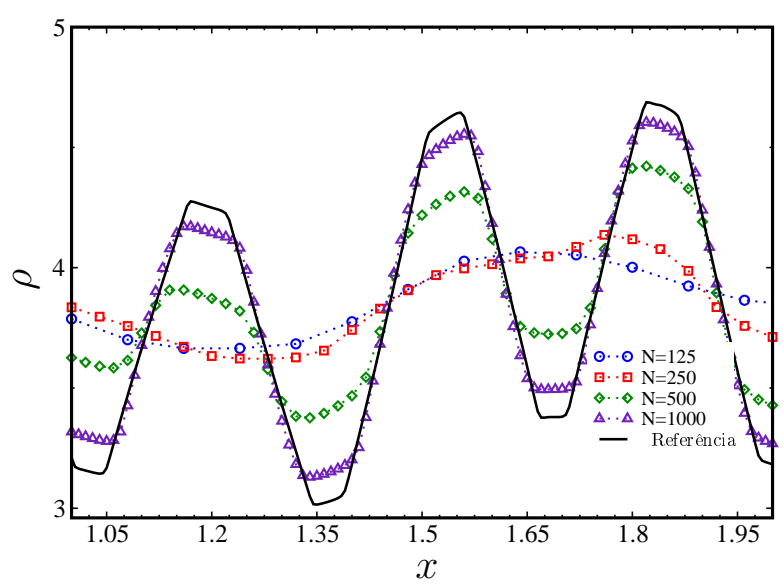

TN

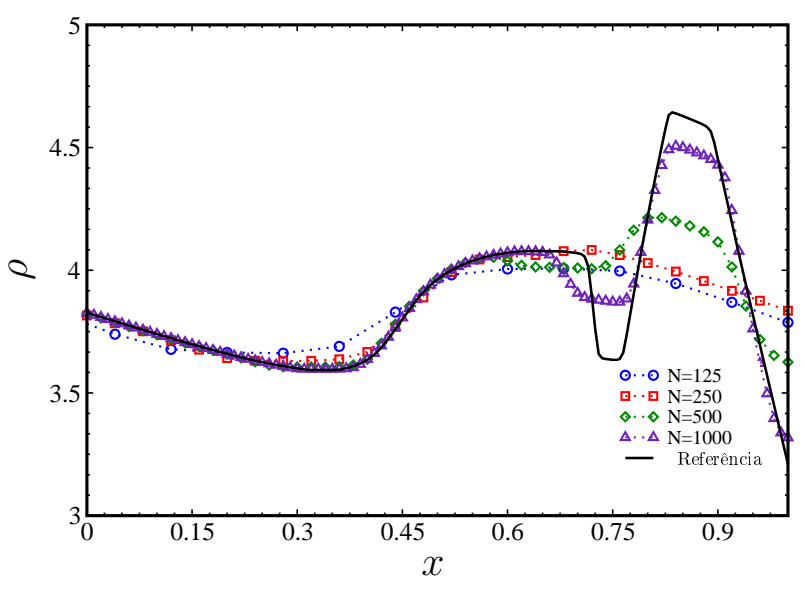

$\mathrm{NF}$

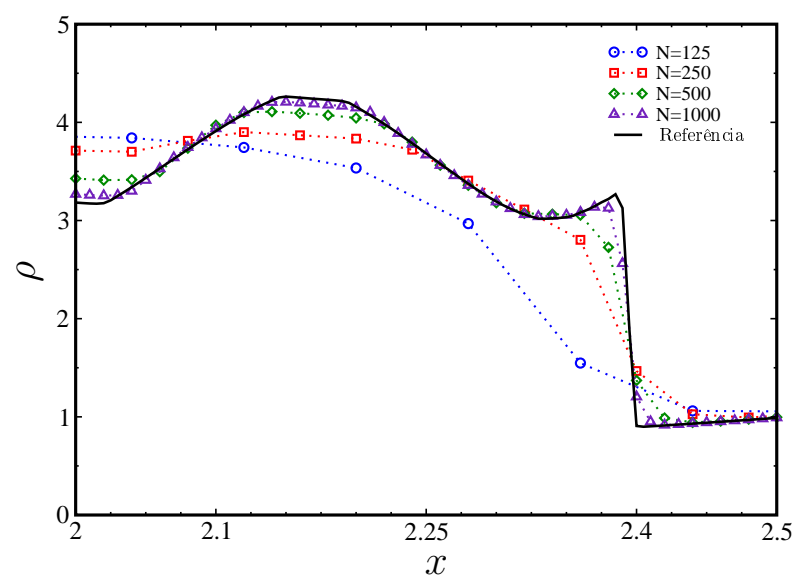

Figura 5.19: Teste 10: soluções numéricas da densidade $\rho$ para o problema de Shu-Osher nas regiões $\mathrm{N}$-wave, transition to $\mathrm{N}$ waves (TN), entropy wave $\mathrm{EW}$ e near field (NF). Ampliações da Fig. 5.18. 
dois diafragmas são rompidos e dois choques são dirigidos ao centro do tubo; rarefações são também direcionadas às extremidades do tubo. Objetiva-se neste teste simular com o esquema TDPUS-C3 a interação entre vários tipos de ondas. Os resultados apresentados a seguir são para a densidade $\rho$ e a componente $u_{1}$ da velocidade no domínio $[0,1]$. A simulação computacional foi rodada numa malha de $N=500$ células computacionais, número de Courant $C F L=0.5$ e a constante do calor específico $\gamma=1.4$. O tempo final de simulação foi de $t=0.038$. As condições iniciais adotadas foram

$$
\left\{\begin{array}{llll}
\rho=1 ; & u_{1}=0 ; & p=1000 ; & 0<x<0.1 \\
\rho=1 ; & u_{1}=0 ; & p=0.01 ; & 0.1<x<0.9 \\
\rho=1 ; & u_{1}=0 ; & p=100 ; & 0.9<x<1 .
\end{array}\right.
$$

Condições refletivas foram impostas no contorno. A solução de referência, calculada com o método de Godunov com termo de correção e o limitador de fluxo MC (van Leer, 1974), foi obtida numa malha de $N=2000$ células computacionais a número de Courant $C F L=0.5$. Uma comparação entre os esquemas estudados e a solução de referência está apresentada na Fig. 5.20, mostrando que os esquemas dependentes do CFL (ADBQUICKEST e ARORA-ROE) fornecem, no geral, os melhores resultados. O esquema TDPUS-C3 forneceu, praticamente, a mesma solução na região de extremo (pico 1); e no pico 2 ele se comportou um pouco pior que ADBQUICKEST e ARORA-ROE. Os esquemas CUBISTA e TOPUS forneceram bons resultados na região do pico 1 e resultados inferiores na região do pico 2, com o CUBISTA um pouco melhor. Nas outras regiões, todos os esquemas comportaram-se de maneira semelhante. A Fig. 5.21 mostra as soluções numéricas dos esquemas TDPUS-C3, EPUS, SDPUS-C1 e TOPUS. Observa-se que nos picos o TDPUS-C3 foi inferior aos esquemas EPUS e SDPUS-C1, sendo o esquema TOPUS o que pior simulou o problema nessas regiões. Nas outras regiões todos os esquemas apresentaram resultados similares.

As Figs. 5.22 e 5.23 apresentam comparações entre os perfis transientes da densidade $\rho$ e da componente $u_{1}$ do campo de velocidades obtidas com os esquemas TDPUS-C3 e SUPERBEE e a solução de referência. O esquema SUPERBEE foi selecionado nesta comparação devido ao seu bom desempenho em pontos extremos (menos difusivo) Roe (1985); também, segundo Hirsch (2007) e Karabasov e Golovizin (2012), este esquema apresenta excelentes propriedades de resolução em descontinuidades em movimento. Como pode ser visto por na Fig. 5.22, a colisão dos choques no tempo $t=0.028$ produz um pico de densidade estreito, acentuado e de difícil captura; esse efeito foi simulado com ambos os esquemas TDPUS-C3 e SUPERBEE. Em particular, resultados teóricos (ver, por exemplo, The Center for Astrophysical Thermonuclear Flashes (2001)) apontam que o pico de densidade tem de ser um pouco menor que 30; o pico calculado com o esquema TDPUS-C3 é $\approx 13.04$ e o com SUPERBEE é $\approx 12.1$. Isto indica que se conseguiu uma melhor aproximação com o uso do esquema TDPUS-C3. 


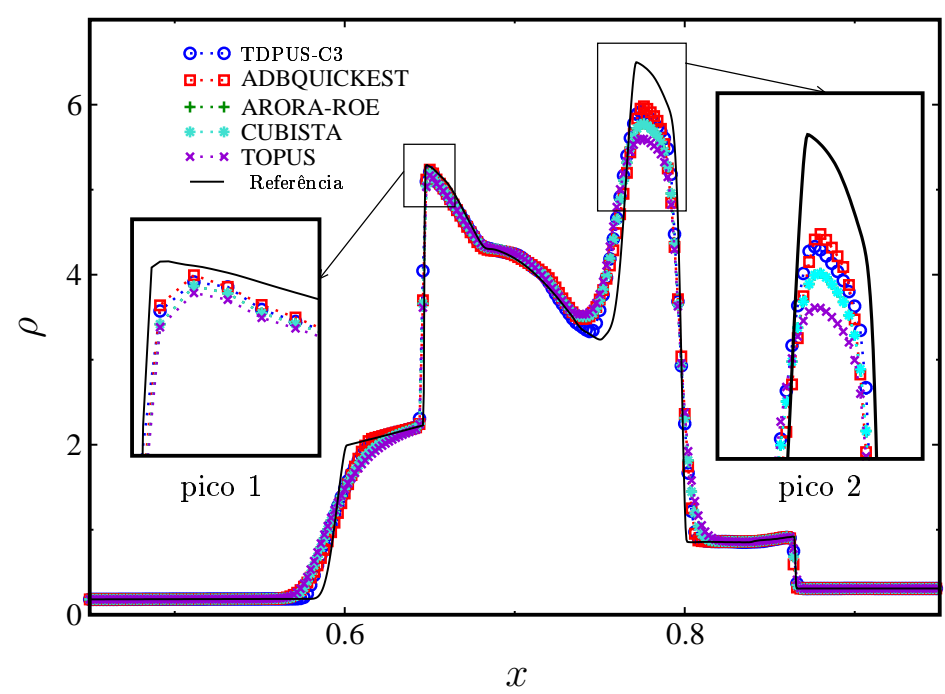

Figura 5.20: Teste 11: comparação das soluções numéricas da densidade $\rho$ para o problema de Woodward-Colella obtidas com TDPUS-C3, ADBQUICKEST, ARORA-ROE, CUBISTA e TOPUS. $t=0.038, N=500$ e $C F L=0.5$.

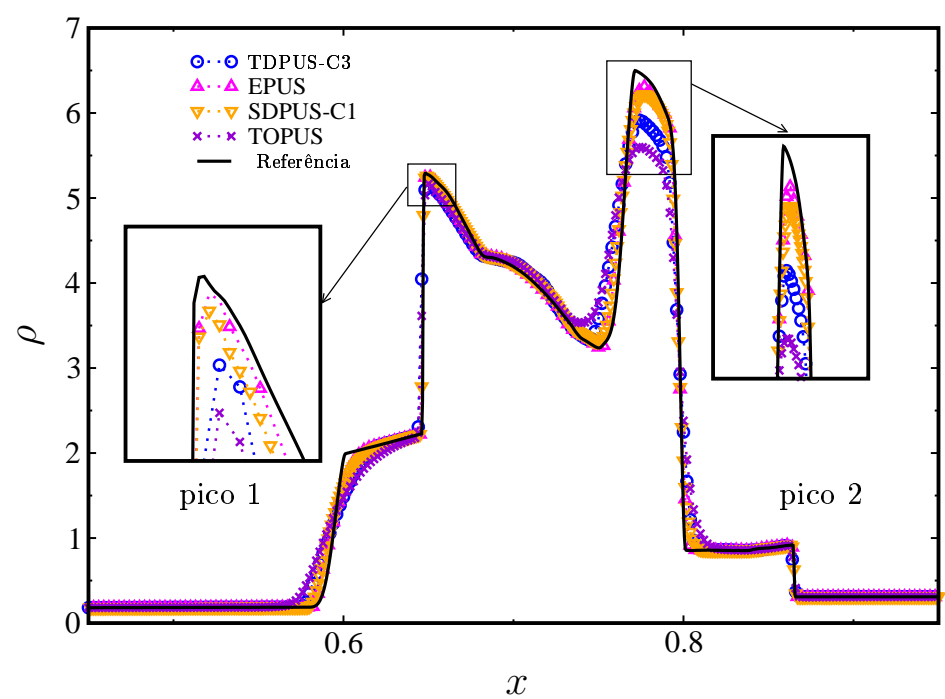

Figura 5.21: Teste 11: comparação das soluções numéricas da densidade $\rho$ para o problema de Woodward-Colella com TDPUS-C3, EPUS, SDPUS-C1 e TOPUS. $t=0.0038, N=500$ e $C F L=0.5$. 

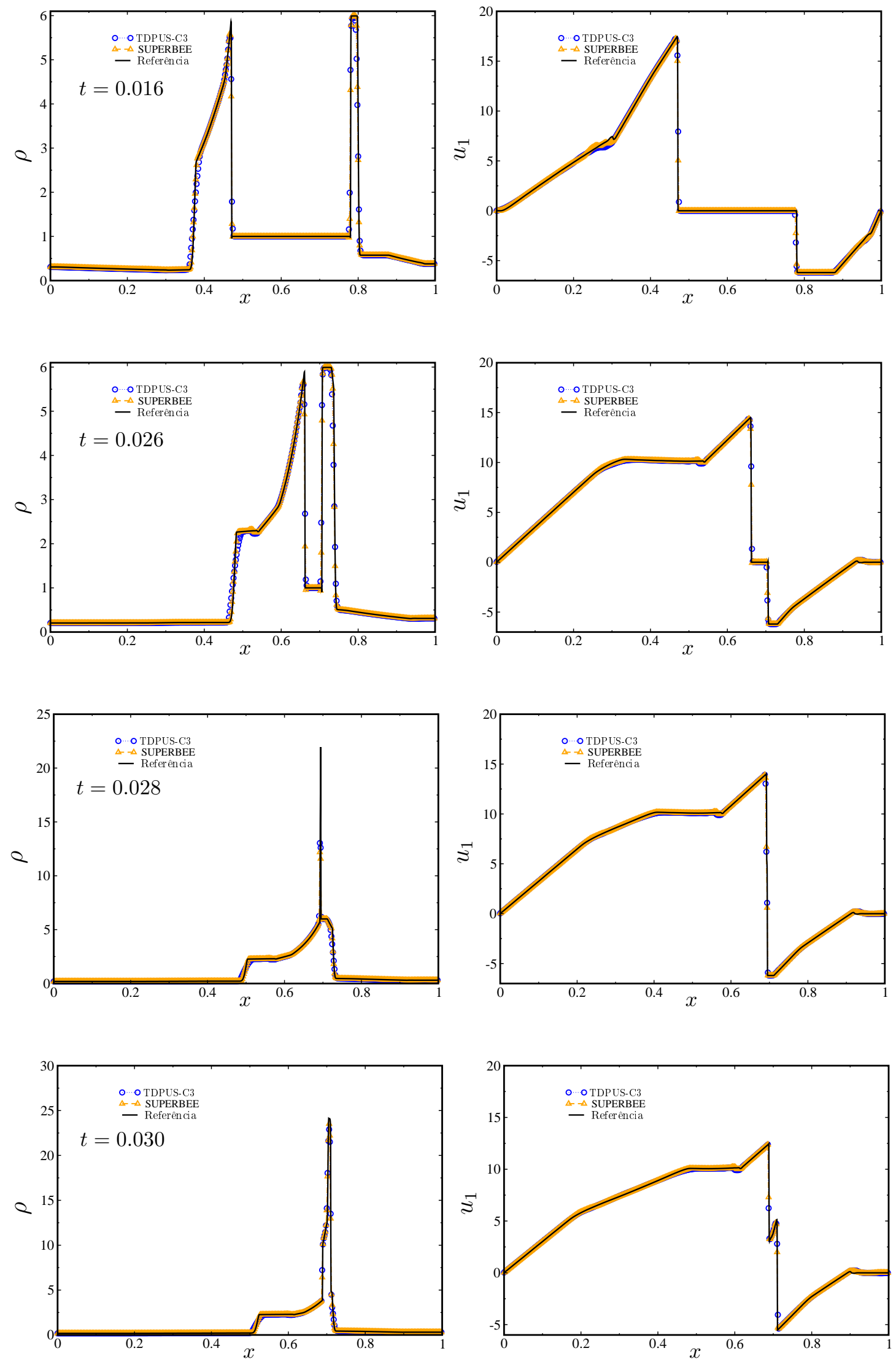

Figura 5.22: Teste 11: problema de Woodward-Colella. Evolução temporal da densidade $\rho$ e velocidade $u_{1}$ das soluções numéricas obtidas com TDPUS-C3 e SUPERBEE. $N=500$ e $C F L=0.5$. 

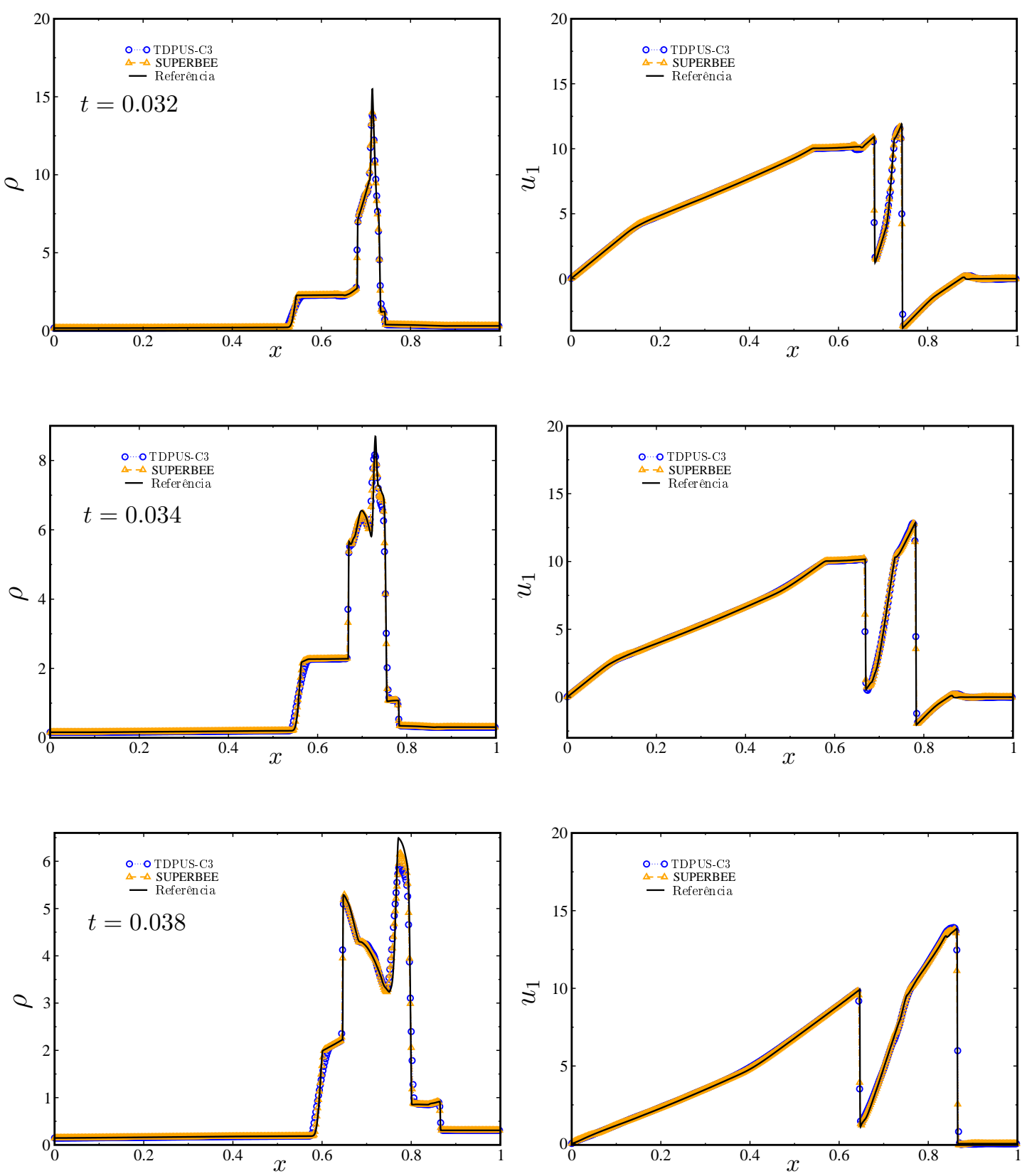

Figura 5.23: Teste 11: problema de Woodward-Colella. Evolução temporal da densidade $\rho$ e velocidade $u_{1}$ obtidas com TDPUS-C3 e SUPERBEE. $N=500$ e $C F L=0.5$. 
O Teste 12, um teste 2D não linear, tem como objetivo acessar a ordem de convergência observada dos métodos numéricos equipados com os esquemas upwind. A simulação computacional foi executada em várias malhas $(20 \times 20,40 \times 40,80 \times 80,160 \times 160$ e $320 \times 320$ células computacionais $)$ no domínio $[0,2] \times[0,2]$, número de Courant $C F L=0.475$, tempo final de simulação $t=2$, e a constante do calor específico $\gamma=1.4$. As condições iniciais consideradas neste teste são

$$
\left\{\begin{array}{l}
\rho(x, y, 0)=1+0.2 \sin (\pi(x+y))) \\
u_{1}(x, y, 0)=0.7 \\
u_{2}(x, y, 0)=0.3 \\
p(x, y, 0)=1
\end{array}\right.
$$

As condições de contorno implementadas foram periódicas.

A solução exata (transiente) do problema (ver Qiu e Shu (2003)) é dada por

$$
\left\{\begin{array}{l}
\left.\rho(x, y, t)=1+0.2 \sin \left(\pi\left(x+y-\left(u_{1}+u_{2}\right) t\right)\right)\right) \\
u_{1}(x, y, 0)=0.7 \\
u_{2}(x, y, 0)=0.3 \\
p(x, y, 0)=1
\end{array}\right.
$$

Apresentam-se nas Tabs. 5.10 e 5.11 os erros e as ordens de convergência observadas nas normas $L_{1}$ e $L_{\infty}$ para a densidade $\rho$. Como pode ser visto por essas tabelas, o método numérico global equipado com o esquema TDPUS-C3 atingiu praticamente a mesma ordem (local) de precisão que os outros métodos, indicando que, neste problema não linear, o novo esquema não é inferior aos esquemas disponíveis na literatura. 
Tabela 5.10: Teste 12: teste de convergência da densidade $\rho$ para as equações não lineares de Euler 2D com TDPUS-C3, ADBQUICKEST, ARORA-ROE, CUBISTA e TOPUS na norma $L_{1} . t=2$ e $C F L=0.475$.

\begin{tabular}{|c|c|c|c|c|c|c|c|c|c|c|}
\hline & \multicolumn{2}{|c|}{ TDPUS-C3 } & \multicolumn{2}{|c|}{ ADBQUICKEST } & \multicolumn{2}{|c|}{ ARORA-ROE } & \multicolumn{2}{|c|}{ CUBISTA } & \multicolumn{2}{|l|}{ TOPUS } \\
\hline Malha & $L_{1}$ & $q$ & $L_{1}$ & $q$ & $L_{1}$ & $q$ & $L_{1}$ & $q$ & $L_{1}$ & $q$ \\
\hline $20 \times 20$ & $9.03 E-03$ & - & $1.33 E-02$ & - & $8.57 E-03$ & - & $8.92 E-03$ & - & $9.53 E-03$ & - \\
\hline $40 \times 40$ & $2.13 E-03$ & 2.08 & $3.35 E-03$ & 1.99 & $2.08 E-03$ & 2.05 & $2.12 E-03$ & 2.07 & $2.24 E-03$ & 2.09 \\
\hline $80 \times 80$ & $5.17 E-04$ & 2.04 & $8.39 E-04$ & 1.99 & $5.10 E-04$ & 2.03 & $5.18 E-04$ & 2.04 & $5.31 E-04$ & 2.07 \\
\hline $160 \times 160$ & $1.27 E-04$ & 2.02 & $2.10 E-04$ & 2.00 & $1.26 E-04$ & 2.01 & $1.27 E-04$ & 2.02 & $1.29 E-04$ & 2.04 \\
\hline $320 \times 320$ & $3.16 E-05$ & 2.01 & $5.26 E-05$ & 2.00 & $3.14 E-05$ & 2.01 & $3.16 E-05$ & 2.01 & $3.18 E-05$ & 2.02 \\
\hline
\end{tabular}

Tabela 5.11: Teste 12: teste de convergência da densidade $\rho$ para as equações não lineares de Euler 2D com TDPUS-C3, ADBQUICKEST, ARORA-ROE, CUBISTA e TOPUS na norma $L_{\infty} . t=2 \mathrm{e}$ $C F L=0.475$.

\begin{tabular}{ccccccccccc}
\hline \hline & \multirow{2}{*}{ TDPUS-C3 } & \multicolumn{2}{c}{ ADBQUICKEST } & \multicolumn{2}{c}{ ARORA-ROE } & \multicolumn{2}{c}{ CUBISTA } & \multicolumn{2}{c}{ TOPUS } \\
\hline Malha & $L_{\infty}$ & $q$ & $L_{\infty}$ & $q$ & $L_{\infty}$ & $q$ & $L_{\infty}$ & $q$ & $L_{\infty}$ & $q$ \\
\hline $20 \times 20$ & $6.88 E-02$ & - & $9.11 E-02$ & - & $6.12 E-02$ & - & $7.06 E-02$ & - & $8.046 E-02$ & - \\
$40 \times 40$ & $3.32 E-02$ & 1.05 & $4.86 E-02$ & 0.91 & $3.09 E-02$ & 0.99 & $3.29 E-02$ & 1.10 & $3.40 E-02$ & 1.24 \\
$80 \times 80$ & $1.61 E-02$ & 1.04 & $2.51 E-02$ & 0.96 & $1.56 E-02$ & 0.99 & $1.61 E-02$ & 1.03 & $1.61 E-02$ & 1.08 \\
$160 \times 160$ & $7.96 E-03$ & 1.02 & $1.28 E-02$ & 0.97 & $7.82 E-03$ & 0.99 & $7.96 E-03$ & 1.02 & $7.96 E-03$ & 1.02 \\
$320 \times 320$ & $3.95 E-03$ & 1.01 & $6.51 E-03$ & 0.98 & $3.92 E-03$ & 1.00 & $3.95 E-03$ & 1.01 & $3.95 E-03$ & 1.01 \\
\hline \hline
\end{tabular}


O Teste 13, conhecido como double Mach reflection-DMR (Woodward e Colella, 1984), consiste em uma onda de choque (a Mach 10) que se desloca horizontalmente e incide numa rampa inclinada com ângulo de $60^{\circ}$. Devido à presença de estruturas complexas (estacionárias e não estacionárias) o problema, além de apresentar um desafio para métodos numéricos, tem sido utilizado com frequência na literatura (ver, por exemplo, Toro e Titarev (2006), Cockburn e Shu (2001), Qiu e Shu (2003), Shi et al. (2003), Zahran (2008) e Castro et al. (2011)) como um caso teste para investigar a influência de duas características importantes dos esquemas convectivos de alta resolução, a saber: (i) minimização de erros de truncamento temporal; e (ii) minimização de viscosidade numérica (dissipação). A Fig. 5.24 ilustra a dissipação introduzida nos cálculos com o uso dos esquemas ADBQUICKEST (mais dissipativo) e WENO5-LW4 de Qiu e Shu (2003)(menos dissipativo).

\section{ADBQUICKEST}

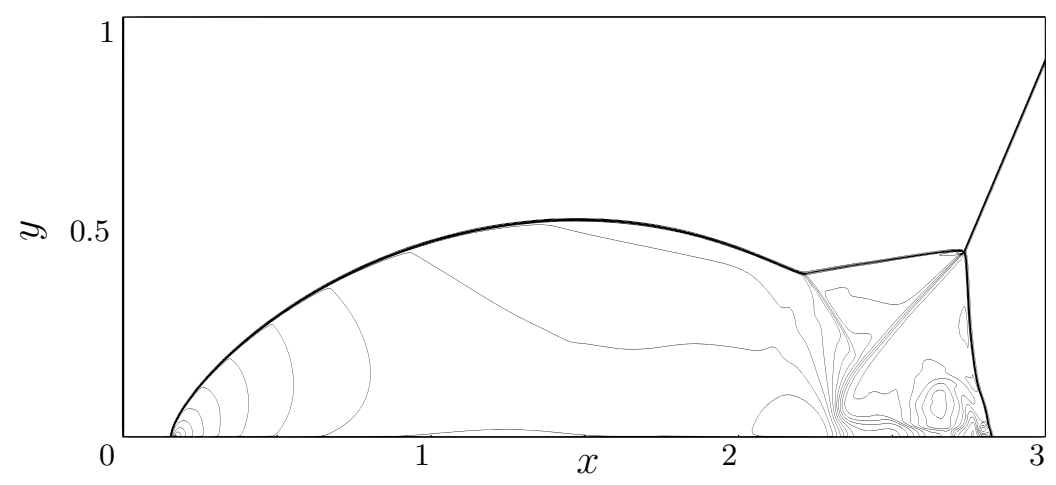

WENO5-LW4

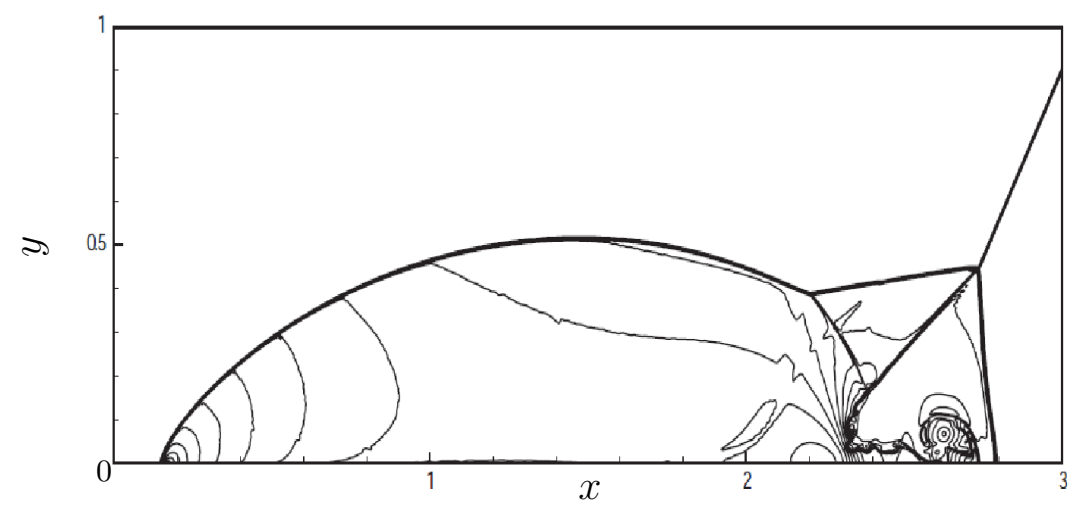

Figura 5.24: Teste 13: soluções numéricas da densidade $\rho$ para o problema DMR com ADBQUICKEST na malha uniforme $\delta x=1 / 400$ e WENO5-LW4 (tomado de Qiu e Shu (2003)) $\delta x=1 / 479$ no tempo $t=0.2$. 30 linhas de contorno de $\rho=1.5$ até $\rho=22.97$. $(x, y) \in[0,4] \times[0,1]$.

Na simulação deste teste foram utilizadas três malhas $(400 \times 100,800 \times 200$ e $1600 \times 400)$ no domínio $[0,4] \times[0,1]$, número de Courant $C F L=0.6$, tempo final de simulação $t=0.2$, e a constante do calor específico $\gamma=1.4$. As condições iniciais adotadas foram

$$
(\rho, u, v, p)= \begin{cases}\left(8,8.25 \cdot \cos \frac{\pi}{6},-8.25 \cdot \sin \frac{\pi}{6}, 116.5\right), & x<x_{0}+\frac{y}{\sqrt{3}} \\ (1.4,0,0,1.0), & x \geq x_{0}+\frac{y}{\sqrt{3}}\end{cases}
$$


com $x_{0}=\frac{1}{6}$. As condições de contorno são como segue: na entrada $(x=0)$, as variáveis de estado são prescritas como $\left(\rho, u_{1}, u_{2}, p\right)^{T}=\left(8,8.25 \cos \frac{\pi}{6},-8.25 \sin \frac{\pi}{6}, 116.5\right)^{T}$; na saída $(x=4)$, extrapolação de ordem zero é imposta; na parede inferior, para $0 \leq x \leq x_{0}$ impõe-se $\left(\rho, u_{1}, u_{2}, p\right)^{T}=$ $\left(8,8.25 \cos \frac{\pi}{6},-8.25 \sin \frac{\pi}{6}, 116.5\right)^{T}$ e para $x_{0} \leq x \leq 4$ condições refletivas; na parede superior admite-se que o escoamento não interage com o choque de forma que as condições impostas são

$$
(\rho, u, v, p)= \begin{cases}\left(8,8.25 \cos \frac{\pi}{6},-8.25 \sin \frac{\pi}{6}, 116.5\right), & 0<x<s(t) \\ (1.4,0,0,1.0), & s(t) \leq x \leq 4\end{cases}
$$

onde $s(t)$ é dada por $s(t)=x_{0}+(1+20 t) / \sqrt{3}$. As soluções numéricas para o problema (na região crítica do domínio) obtidas com os esquemas upwind usando Euler explícito para a marcha no tempo estão mostradas nas Figs. 5.25 e 5.26. Para comparações, a Fig. 5.27 mostra a solução numérica numa malha de $3840 \times 960$ células computacionais obtida com o esquema WENO5 de Shi et al. (2003) usando um método de Runge-Kutta de terceira ordem TVD para a marcha no tempo, e a obtida com o esquema SUPERBEE numa malha $800 \times 200$ células computacionais. É importante comentar que esta apresentou de instabilidade numérica na malha de $1600 \times 400$ células.

Com base nas Figs. 5.25 e 5.26, mais a Fig. 5.27 mostrando comparações, no geral, pode-se classificar com respeito à introdução de dissipação os esquemas estudados na seguinte ordem (do que introduz mais viscosidade numérica para o que introduz menos): ADBQUICKEST, ARORA-ROE, CUBISTA, TOPUS e TDPUS-C3. Em particular, o esquema CUBISTA na malha mais fina (1600 $\times$ 400) introduziu mais "ruídos", em comparação com os demais esquemas. A solução obtida com o esquema TDPUS-C3, nas malhas $800 \times 200$ e $1600 \times 400$, contempla mais estruturas vorticais de escalas médias que os outros esquemas, quando comparada à solução de referência com WENO5. Além disso, a solução com o esquema TDPUS-C3 na malha $800 \times 200$ é comparável com a solução obtida com o TOPUS na malha mais fina. É importante destacar neste ponto que: (i) o esquema TDPUS-C3, mesmo combinado com Godunov de primeira ordem no tempo, captura algumas estruturas (vorticais) complexas no problema double Mach reflection, da mesma forma como faz o esquema WENO5 que tem terceira ordem no tempo e quinta ordem no espaço; e (ii) as estruturas transientes (àquelas entre $2.2 \leq x \leq 3$ e $0 \leq y \leq 0.5$ ) fornecidas pelos esquemas estudados (com exceção do ADBQUICKEST e ARORA-ROE) apareceram porque o método de Godunov tem a particularidade de avançar mais o choque (ver Woodward e Colella (1984)). 

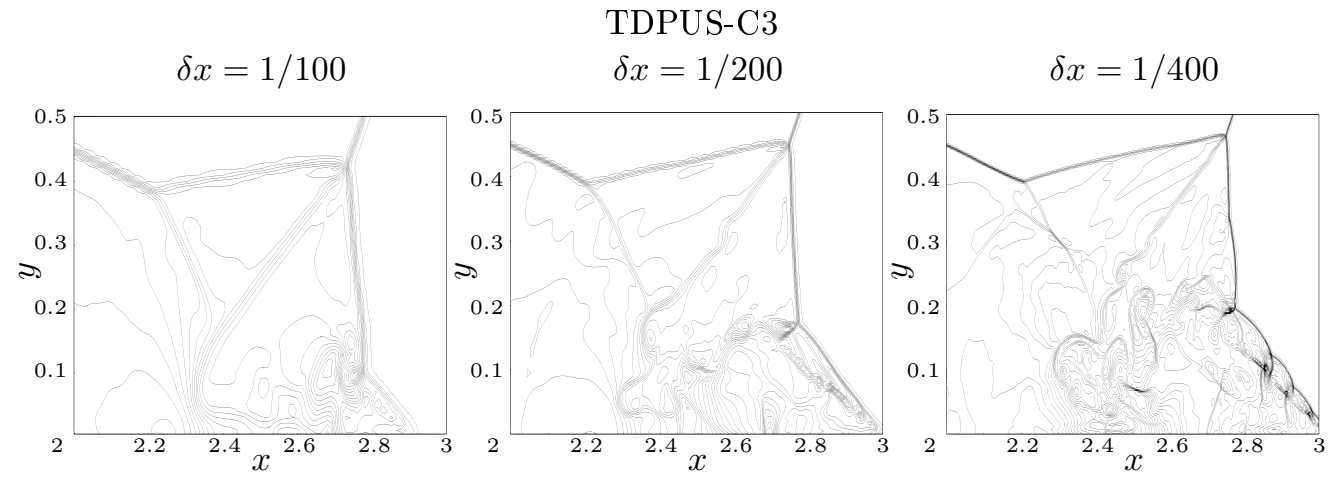

ADBQUICKEST
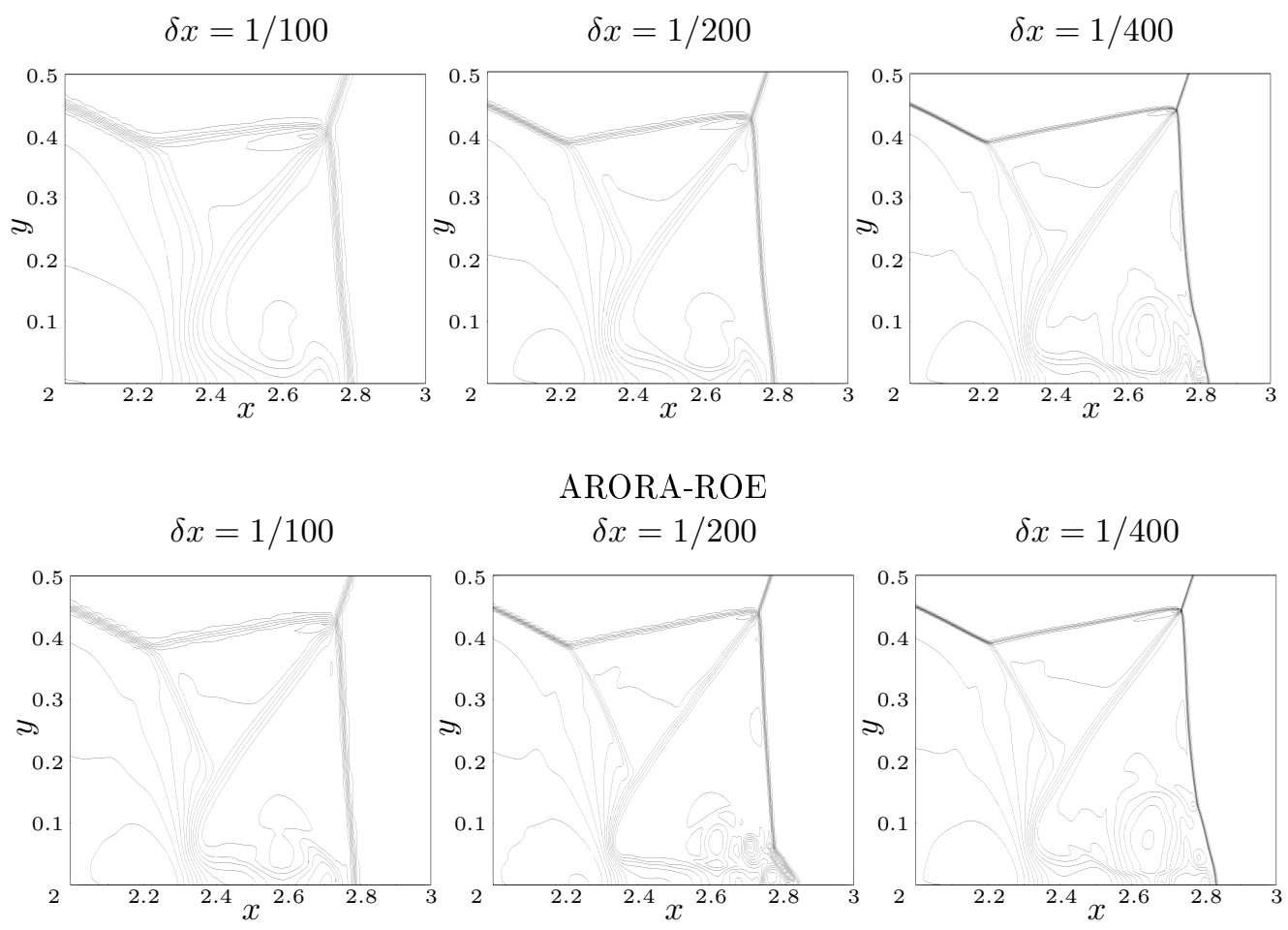

Figura 5.25: Teste 13: ampliação da região principal das soluções numéricas para a densidade $\rho$ para o problema DMR com TDPUS-C3, ADBQUICKEST e ARORA-ROE. 30 linhas de contorno de $\rho=1.5$ até $\rho=22.97$, com malhas uniformes $\delta x=1 / 100,1 / 200,1 / 400, t=0.2$ e $C F L=0.6$. 


\section{CUBISTA}
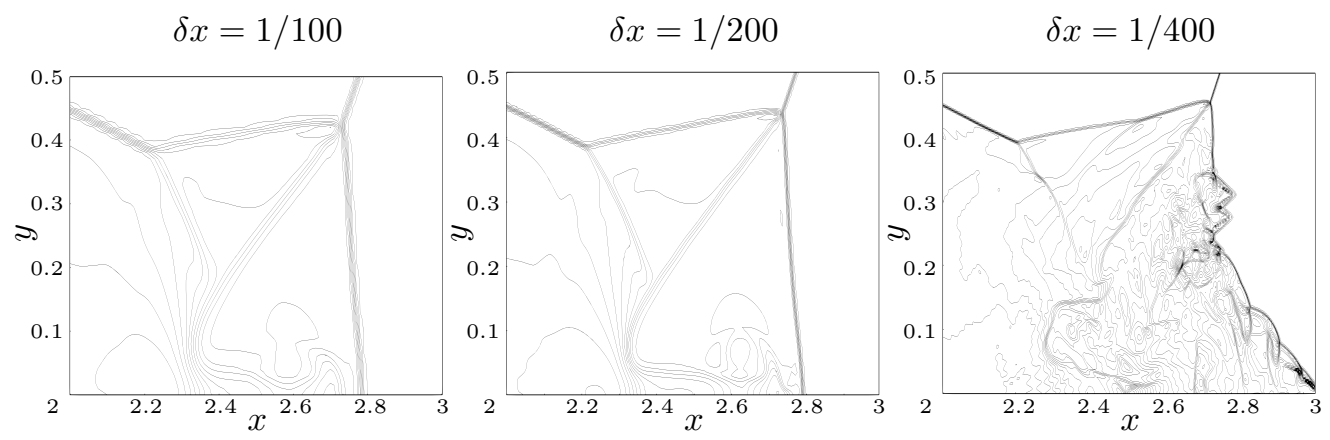

\section{TOPUS}
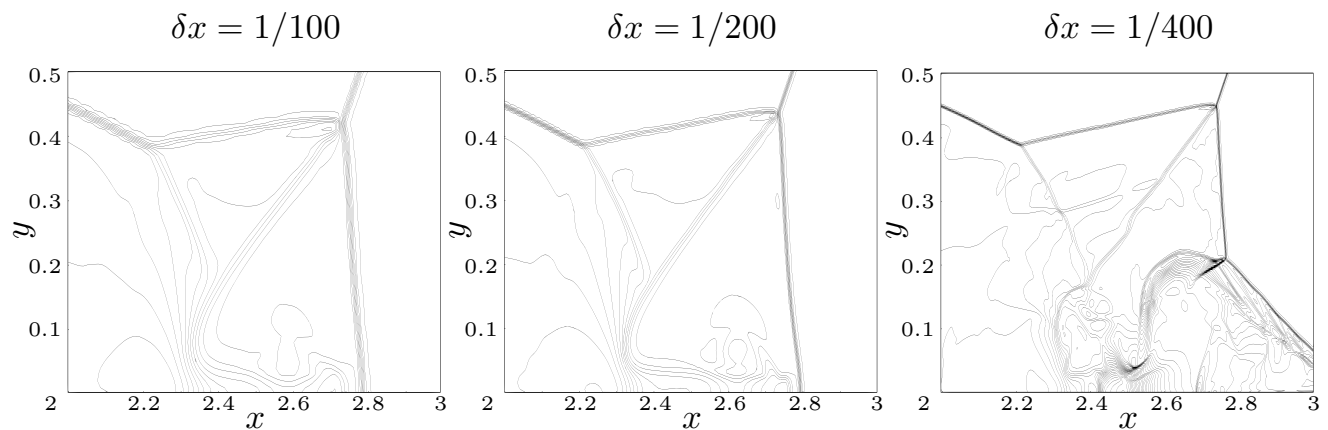

Figura 5.26: Teste 13: ampliação da região principal das soluções numéricas para a densidade $\rho$ do problema DMR no tempo $t=0.2$ calculadas com CUBISTA e TOPUS. 30 linhas de contorno de $\rho=1.5$ até $\rho=22.97$, com malhas uniformes $\delta x=1 / 100,1 / 200,1 / 400, t=0.2$ e $C F L=0.6$.
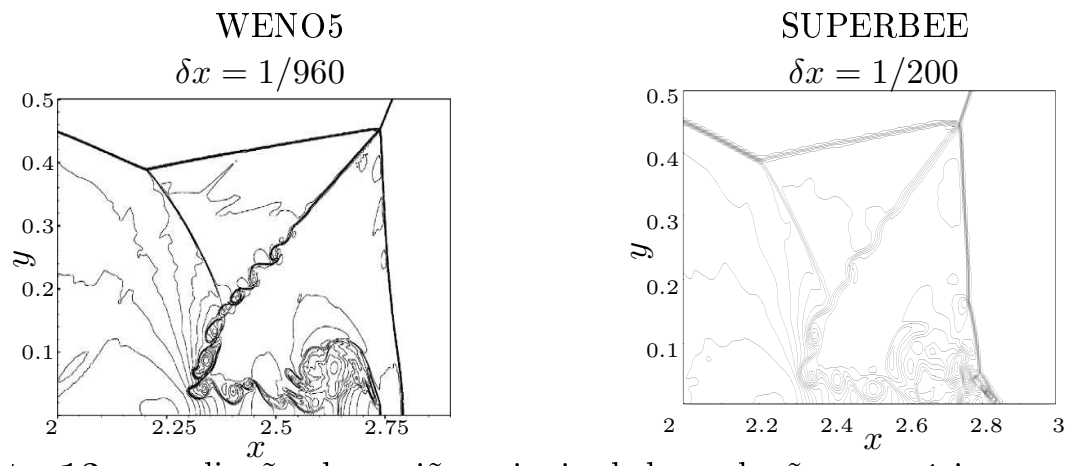

Figura 5.27: Teste 13: ampliação da região principal das soluções numéricas para a densidade $\rho$ do problema DMR no tempo $t=0.2$ calculada com SUPERBEE e $\delta x=1 / 200$ e WENO5 de (Jiang e Shu, 1996) e $\delta x=1 / 960$ (Extraído de Shi et al. (2003)). 30 linhas contorno de $\rho=1.5$ até $\rho=22.97$. $t=0.2$ e $C F L=0.6$. 
No Teste 14, investiga-se o desempenho dos esquemas upwind em resolver o problema conhecido como instabilidades de Rayleigh-Taylor (ver, por exemplo, Chandrasekhar (1961) e Sharp (1984)). Essa instabilidade ocorre, por exemplo, quando há superposição de dois fluidos (um mais pesado e um mais leve) sob a ação do campo gravitacional. Perturbações iniciais na interface que separa os dois fluidos são amplificadas e tendem a crescer com o tempo levando a formação de bolhas e espigões, cada um dos quais penetrando uns nos outros, seguido do desenvolvimento de uma camada de mistura complexa. O problema representa um importante teste para códigos em DFC e, como no problema de double Mach reflection, pode ser usado para medir a introdução (intrínseca) de dissipação do método numérico (Samtaney e Pullin, 1996). Para a simulação deste problema complexo, a interface está posicionada em $y=\frac{1}{2}$ e o fluido mais pesado está abaixo da interface com densidade $\rho_{\text {pesado }}=2$ e o fluido mais leve acima da interface com $\rho_{\text {leve }}=1$; a pressão é contínua através da interface e uma perturbação na componente de velocidade $u_{2}$ é injetada. As condições iniciais (ver Fig. 5.28) são então dadas por

$$
(\rho, u, v, p)= \begin{cases}(2,0,-0.025 c \cdot \cos (8 \pi x), 2 y+1), & 0 \leq y<\frac{1}{2} \\ \left(1,0,-0.025 c \cdot \cos (8 \pi x), y+\frac{3}{2}\right), & \frac{1}{2} \leq y<1\end{cases}
$$

onde $c=\sqrt{\frac{\gamma p}{\rho}}$ é a velocidade do som e $\gamma=5 / 3$.

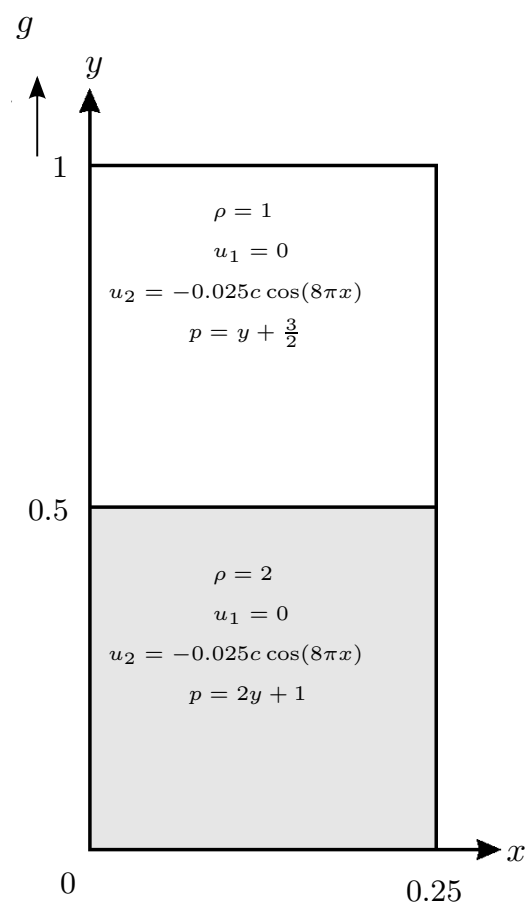

Figura 5.28: Teste 14: condições iniciais do problema das instabilidades de Rayleigh-Taylor.

Os cálculos foram feitos no domínio $\left[0, \frac{1}{4}\right] \times[0,1]$ no tempo final de simulação $t=1.95$ e a número de Courant $C F L=0.6$. Uma malha de $240 \times 960$ células computacionais foram usadas nos experimentos 
numéricos. As condições de contorno aplicadas são: condições refletivas nas laterais direita e esquerda; $\rho=\rho_{\text {leve }}=1, p=2.5, u_{1}=u_{2}=0$ no topo; $\rho=\rho_{\text {pesado }}=2, p=1, u_{1}=u_{2}=0$ na seção inferior. A constante gravitacional é tomada como $g=1$. Uma vez que não se conhece uma solução analítica para o problema, compara-se qualitativamente os resultados numéricos com os fornecidos pela literatura (Shi et al., 2003). A Fig. 5.29 mostra a posição da interface em dois tempos calculada com os esquemas TDPUS-C3, ADBQUICKEST, ARORA-ROE, CUBISTA e TOPUS. Também, para comparações, nesta mesma figura, são apresentados os resultados numéricos com os esquemas SUPERBEE e WENO5 de Jiang e Shu (1996). Pode ser visto por essa figura que, quando comparado com outros esquemas (exceto SUPERBEE e WENO5), o esquema TDPUS-C3 fornece o melhor resultado. Os resultados com ARORA-ROE, CUBISTA e TOPUS são semelhantes, com o TOPUS um pouco mais dissipativo. O esquema ADBQUICKEST mostrou ser o mais dissipativo, produzindo o pior resultado. Em síntese, pode-se inferir que o esquema TDPUS-C3 introduz pouca dissipação, como fazem os esquemas SUPERBEE e WENO5. Outro ponto importante a ser notado é que o esquema TDPUS-C3 foi implementado com uma marcha no tempo via Euler explícito, ao passo que o WENO5 emprega um método de Runge-Kutta de terceira ordem de precisão; isto permite concluir que o método de Godunov com termo de correção equipado com TDPUS-C3 pode ser visto como uma alternativa para simular problemas complexos em DFC.

Com o intuito de realizar uma comparação quantitativa entre os resultados numéricos fornecidos com o uso dos esquemas upwind, apresenta-se na Fig. 5.30 a energia cinética $e_{c}=\frac{1}{2}\left[\rho\left(u_{1}^{2}+u_{2}^{2}\right)\right]$ ao longo da reta $y=4 x$ obtida com todos os esquemas no tempo $t=1.95$. Esses dados quantificam os observados nos resultados da Fig. 5.29.

Na Fig. 5.31 apresentam-se a posição da interface dos esquemas TDPUS-C3, EPUS, SDPUS-C1 e TOPUS nos tempos $t=1.365$ e 1.95. Observa-se a partir dessas figuras que o esquema TDPUS-C3 apresenta menos dissipação que os outros esquemas. Conclui-se que o TDPUS-C3 é superior para este tipo de problema apresentando fortes estruturas vorticais com relação aos esquemas TOPUS, SDPUS-C1 e EPUS. 
TDPUS-C3
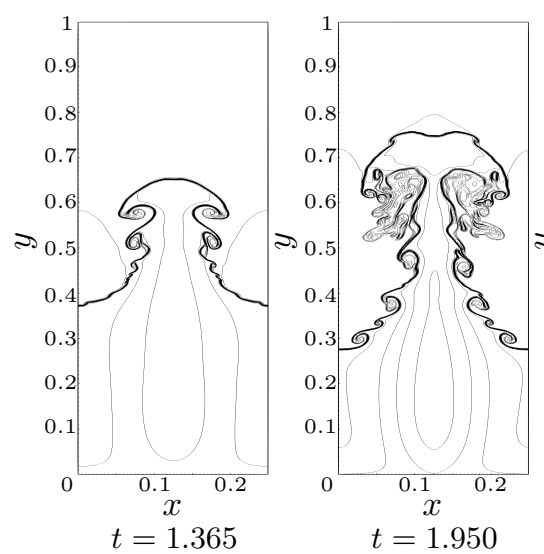

ARORA-ROE

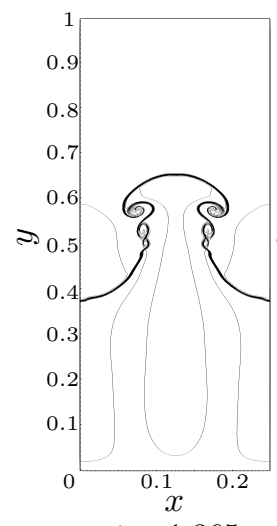

$t=1.365$
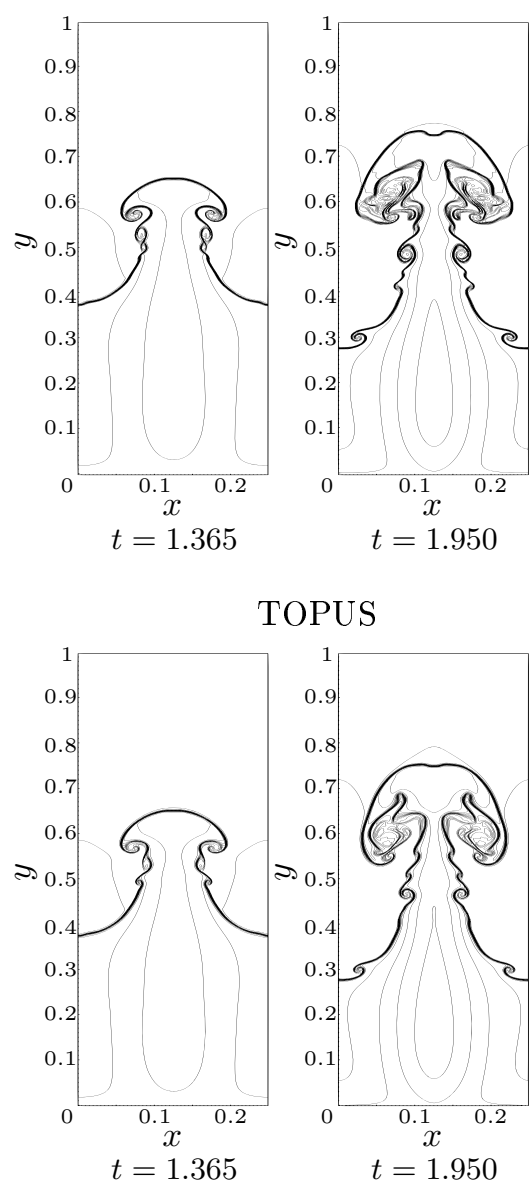

TOPUS

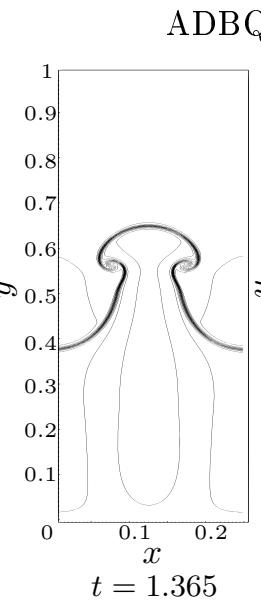

ADBQUICKEST

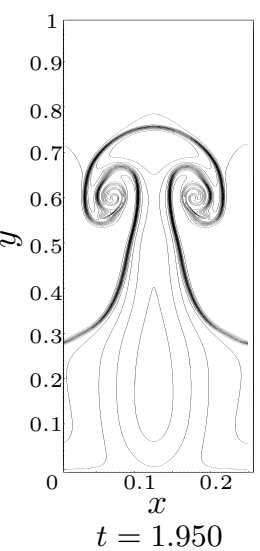

CUBISTA
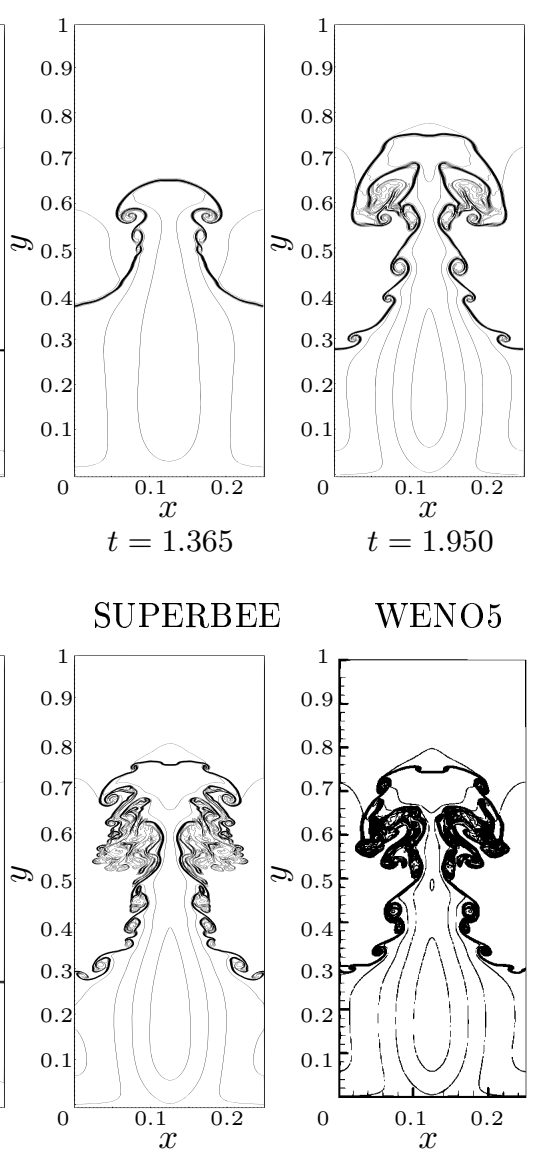

$t=1.950$

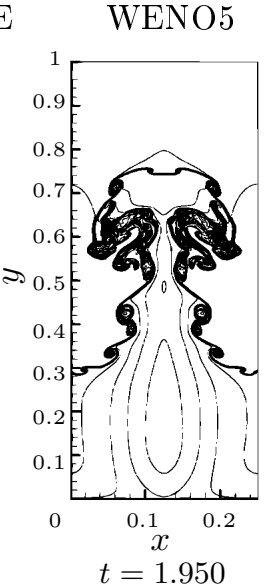

Figura 5.29: Teste 14: soluções numéricas para a densidade $\rho$ do problema de Rayleigh-Taylor com TDPUS-C3, ADBQUICKEST, ARORA-ROE, CUBISTA, TOPUS, SUPERBEE e WENO5 (tomado de Shi et al. (2003). $\delta x=1 / 960,15$ linhas de contorno com valores de $\rho=0.952269$ até $\rho=2.14589$. $t=1.95$ e $C F L=0.6$. 

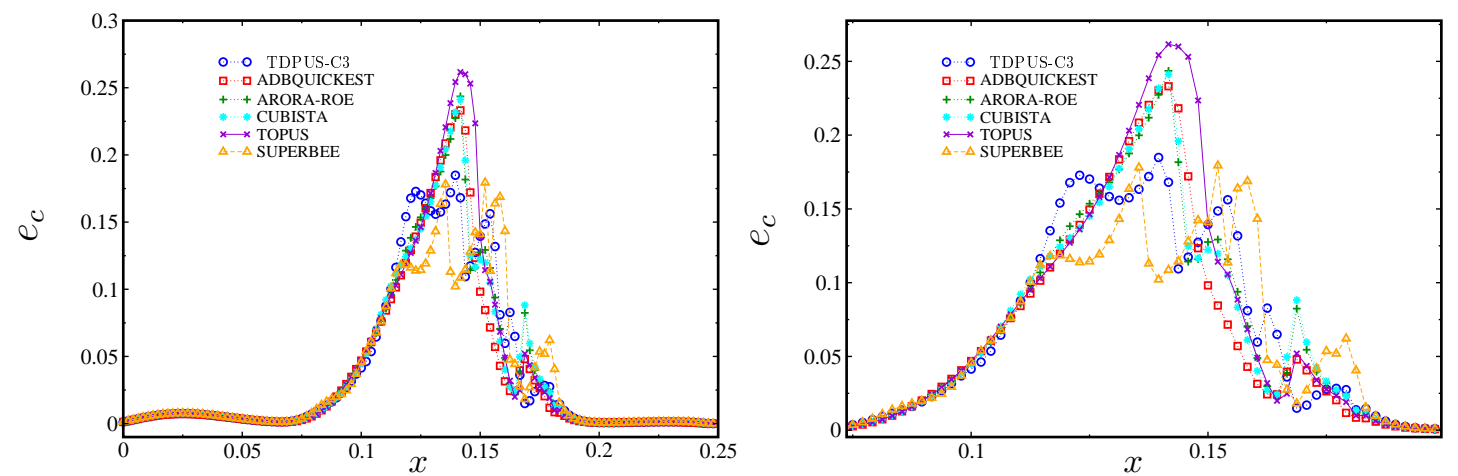

Figura 5.30: Teste 14: Instabilidades de Rayleigh-Taylor. Perfil da energia cinética em $y=4 x$ no tempo $t=1.95$ obtidos com TDPUS-C3, ADBQUICKEST, ARORA-ROE, CUBISTA, TOPUS e SUPERBEE. À direita apresenta-se a ampliação do perfil estudado.

TDPUS-C3
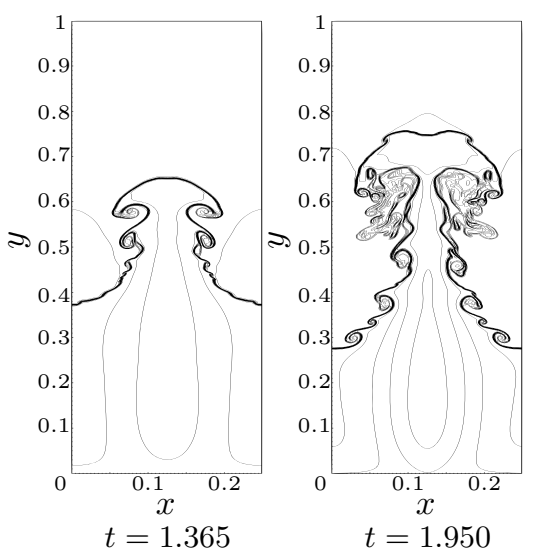

SDPUS-C1
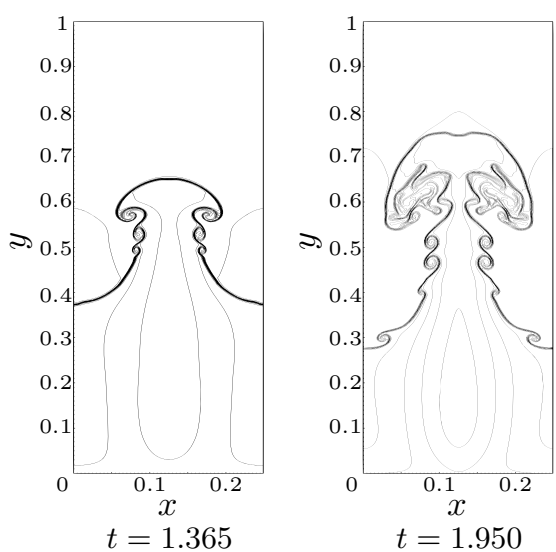

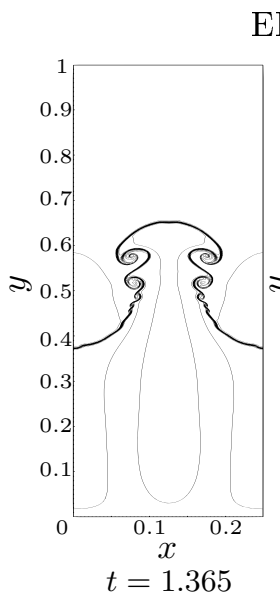

EPUS

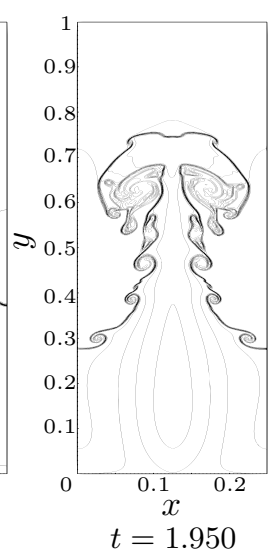

TOPUS
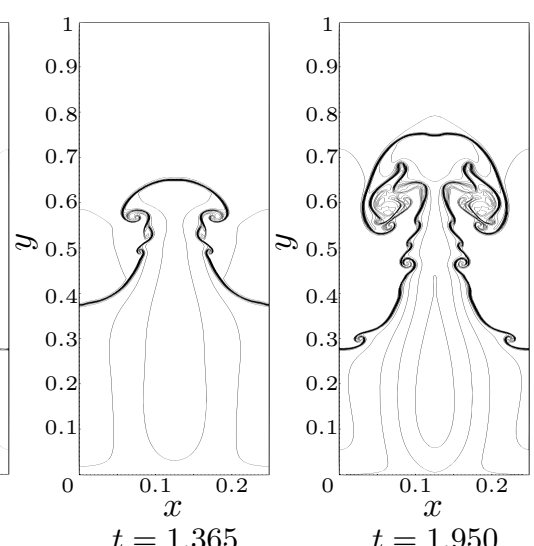

Figura 5.31: Teste 14: soluções numéricas para a densidade $\rho$ do problema das instabilidades de Rayleigh-Taylor com TDPUS-C3, EPUS, SDPUS-C1 e TOPUS. $\delta x=1 / 960,15$ linhas de contorno com valores de $\rho=0.952269$ até $\rho=2.14589 . t=1.95$ e $C F L=0.6$. 


\subsubsection{Equações da magnetohidrodinâmica}

A interação de um plasma com um campo magnético, a qual produz choques e estruturas vorticais complexas, é simulada nesta seção. Essa interação é modelada pelas equações da magnetohidrodinâmica (MHD) dadas pela Eq. (3.1) com variáveis de estado e funções fluxo

$$
\left\{\begin{array}{l}
\boldsymbol{u}=\left(\rho, \rho u_{1}, \rho u_{2}, \rho u_{3}, B_{1}, B_{2}, B_{3}, E\right)^{T} \\
\boldsymbol{f}(\boldsymbol{u})=\left(\rho u_{1}, \rho u_{1}^{2}+p^{*}-0.5 B_{1}^{2}, \rho u_{1} u_{2}-B_{1} B_{2}, \rho u_{1} u_{3}-B_{1} B_{3}, 0, B_{2} u_{1}-B_{1} u_{2},\right. \\
\left.B_{3} u_{1}-B_{3} u_{3},(E+p *) u_{1}-(\boldsymbol{u} \cdot \boldsymbol{B}) B_{1}\right)^{T} \\
\boldsymbol{g}(\boldsymbol{u})=\left(\rho u_{2}, \rho u_{1} u_{2}-B_{1} B_{2}, \rho u_{2}^{2}+p^{*}-0.5 B_{3}^{2}, \rho u_{1} u_{3}+B_{1} B_{3}, B_{1} u_{2}-B_{2} u_{1},\right. \\
\left.0, u_{2} B_{3}-u_{3} B_{2},(E+p *) u_{2}-(\boldsymbol{u} \cdot \boldsymbol{B}) B_{2}\right)^{T} .
\end{array}\right.
$$

A quantidade $u_{3}$ é a componente de velocidade na direção $z$ e $\boldsymbol{B}=\left(B_{1}, B_{2}, B_{3}\right)^{T}$ representa o campo magnético. A energia total é dada por $E=\frac{1}{2}\left(\rho|\boldsymbol{u}|^{2}+|\boldsymbol{B}|^{2}\right)+\frac{p}{\gamma-1}$ e $p *=p+0.5|\boldsymbol{B}|^{2}$ é a pressão total. As outras quantidades foram definidas previamente. Adicionalmente, o campo magnético deve satisfazer a restrição de divergência livre $\nabla \cdot \boldsymbol{B}=0$ (ver, por exemplo, Evans e Hawley (1988)). Quando comparadas às equações de Navier-Stokes, as equações da magnetohidrodinâmica são mais complexas desde que elas são propensas ao aparecimento de uma família de ondas que se propagam em diferentes velocidades e de maneira caótica. Dois experimentos numéricos foram realizados: um no caso 1D (Teste 15) e um no caso 2D (Teste 16).

Para o Teste 15, conhecido como problema de Brio e Wu (1988), os cálculos foram feitos no domínio $[-1,1]$, no tempo final de simulação 0.2 e ao número de Courant $C F L=0.8$. Uma malha de 800 células computacionais foi usada no experimento. As condições inicias são as mesmas adotadas em Brio e Wu (1988) e Barmin et al. (1996) e são definidas por

$$
\left(\rho, u_{1}, u_{2}, u_{3}, B_{1}, B_{2}, B_{3}, p\right)^{T}= \begin{cases}(1,0,0,0,0.75,1,0,1)^{T}, & x \leq 0 \\ (0.125,0,0,0,0.75,-1,0,0.1)^{T}, & x>0\end{cases}
$$

com $\gamma=2$. As condições de contorno aplicadas são tipo extrapolação de ordem zero. Uma vez que não se conhece uma solução analítica para o problema, compara-se os resultados numéricos com a solução de referência calculada numa malha de 10000 células computacionais usando-se o método de Godunov com termo de correção e o limitador de fluxo MC (van Leer, 1974). Na Fig. 5.32 estão representadas as soluções numéricas (com o esquema TDPUS-C3) e de referência para a densidade no problema de Brio-Wu, onde pode ser visto que, no global, o esquema TDPUS-C3 conseguiu capturar com sucesso todas as ondas e descontinuidades.

O Teste 16, conhecido como problema de Orszag e Tang (1979), possui características significativas da turbulência em magnetohidrodinâmica e tem sido considerado um problema desafiador na literatura especializada (ver, por exemplo, Dahlburg e Picone (1989), Jiang e Wu (1999) ou Balbás et al. (2004)) para estimar a precisão local de um esquema upwind de alta resolução. Para a simulação deste problema complexo, o domínio computacional é definido como $[0,2 \pi] \times[0,2 \pi]$ com condições de 


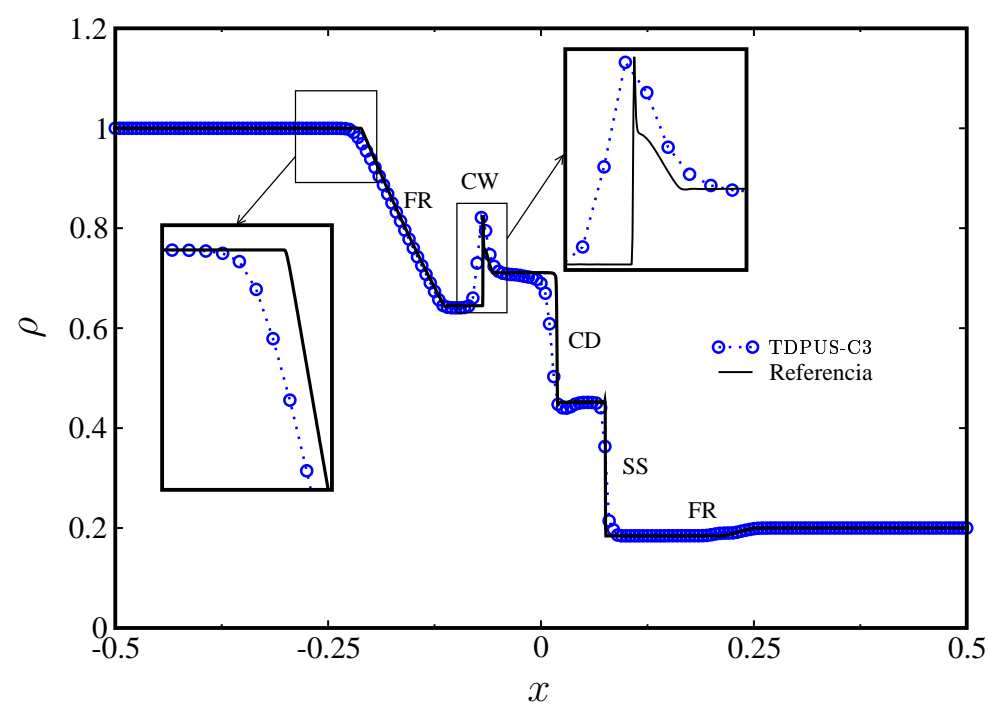

Figura 5.32: Teste 15: soluções numéricas da densidade $\rho$ do problema da MHD 1D com TDPUS-C3. $t=0.2, N=800$ e $C F L=0.8$. Onda de rarefação (FR), onda composta (CW), descontinuidade de contato (CD) e onda de choque pequena (SS).

fronteira periódicas e condições iniciais dadas por

$$
\begin{aligned}
& u_{1}(x, y)=-\sin y, \quad u_{2}=\sin x, \quad u_{3}=0 ; \quad \rho=\gamma^{2}, \\
& B_{1}(x, y)=-\sin y, \quad B_{2}(x, y)=\sin 2 x, \quad B_{3}=0 ; \quad p=\gamma=5 / 3 .
\end{aligned}
$$

Várias malhas computacionais foram utilizadas na simulação, a saber: $16 \times 16,32 \times 32,64 \times 64$, $128 \times 128$ e $256 \times 256$, a número de Courant $C F L=0.75$ e três tempos finais $(t=0.25,0.5$ e 3$)$. A Fig. 5.33, casos (a) e (b), mostra a densidade calculada na malha $256 \times 256$ e no tempo $t=0.25$ com o esquema TDPUS-C3; o caso (a) ilustra a superfície $\rho=\rho(x, y, z)$ global e o caso (b) a projeção desta no plano $x y$. Dessa figura pode ser visto os resultados com o esquema TDPUS-C3 são comparáveis com resultados da literatura Dahlburg e Picone (1989), Jiang e Wu (1999) e Balbás et al. (2004). A Fig. 5.34 apresenta uma comparação dos resultados obtidos com TDPUS-C3 e o esquema centrado de Balbás et al. (2004) a $C F L=0.4$ para o perfil de pressão ao longo da reta $y=0.625 \pi$, numa malha de $128 \times 128$ células e tempo $t=3$. Mais uma vez, essa comparação confirma que o esquema TDPUS-C3 gera resultados comparáveis com o da literatura.

Para finalizar, apresenta-se na Tab. 5.12 os erros e ordens de convergência observadas, na norma $L_{1}$, para todos os esquemas upwind estudados com tempo final de simulação $t=0.5$. Os dados dessa tabela mostram que todos os esquemas superaram ordem dois, com TDPUS-C3 apresentando ligeiramente ordem superior 


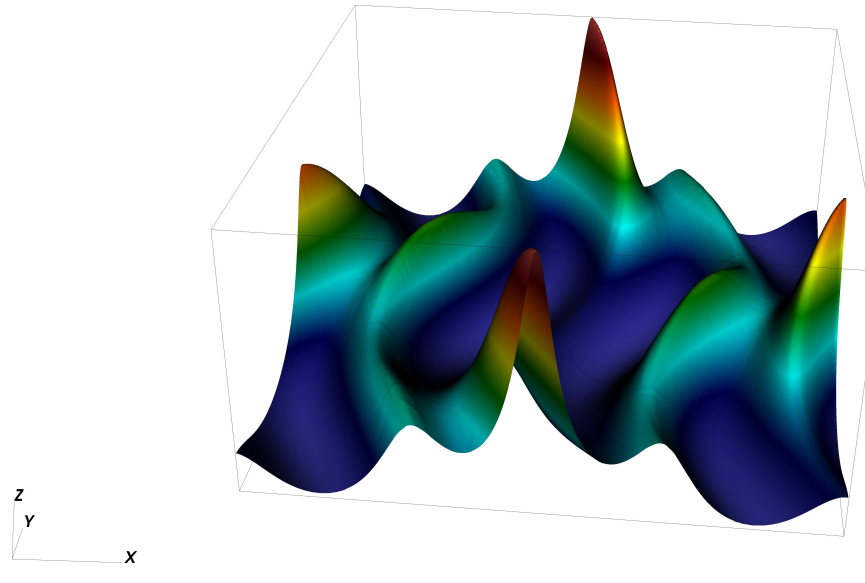

(a)

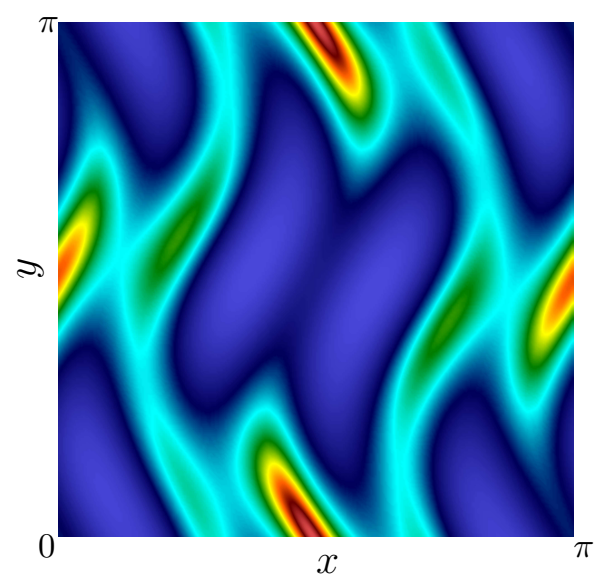

(b)

Figura 5.33: Teste 16: (a) superfície da densidade $\rho$ para o problema de Orszag-Tang, (b) densidade $\rho$ no plano $x y . t=0.25,256 \times 256$ células computacionais e $C F L=0.75 .12$ linhas de contorno para a densidade; vermelho-maior valor (5.278), azul- menor valor (2.176).

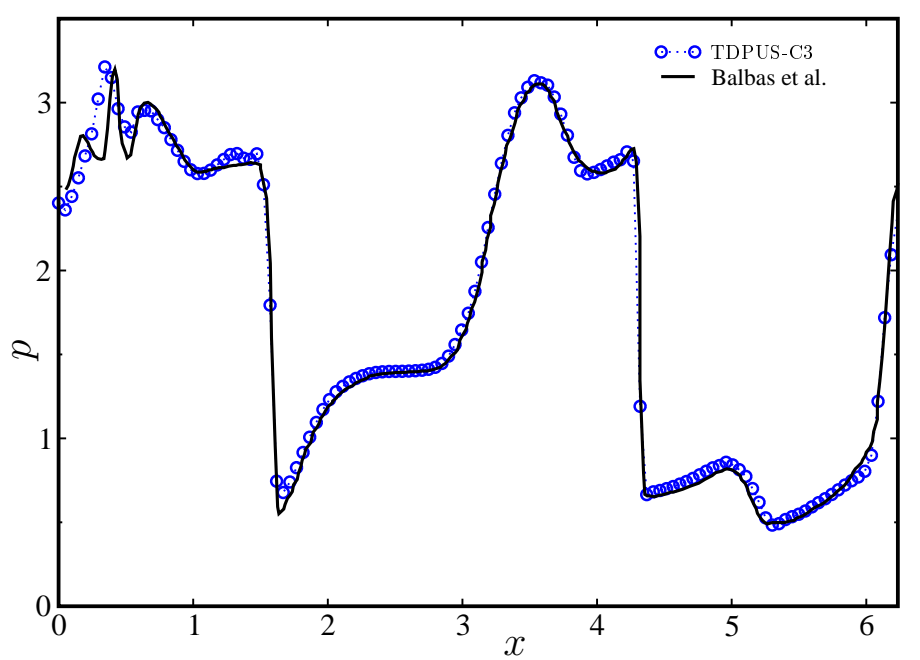

Figura 5.34: Teste 18: perfil da pressão $p$ ao longo da linha $y=0.625 \pi . t=3,128 \times 128$ células computacionais e $C F L=0.75$.

Tabela 5.12: Teste 18: teste de convergência da densidade $\rho$ para o problema de Orszag-Tang com TDPUS-C3, ADBQUICKEST, ARORA-ROE, CUBISTA e TOPUS na norma $L_{1} . t=0.5$ e $C F L=$ 0.75 .

\begin{tabular}{|c|c|c|c|c|c|c|c|c|c|c|}
\hline & \multicolumn{2}{|c|}{ TDPUS-C3 } & \multicolumn{2}{|c|}{ ADBQUICKEST } & \multicolumn{2}{|c|}{ ARORA-ROE } & \multicolumn{2}{|c|}{ CUBISTA } & \multicolumn{2}{|l|}{ TOPUS } \\
\hline Malha & $L_{1}$ & $q$ & $L_{1}$ & $q$ & $L_{1}$ & $q$ & $L_{1}$ & $q$ & $L_{1}$ & $q$ \\
\hline $16 \times 16$ & $1.08 E+00$ & - & $1.08 E+00$ & - & $1.07 E+00$ & - & $1.07 E+00$ & - & $1.06 E+00$ & - \\
\hline $32 \times 32$ & $2.77 E-01$ & 1.97 & $2.77 E-01$ & 1.96 & $4.46 E-01$ & 1.27 & $2.74 E-01$ & 1.97 & $2.72 E-01$ & 1.97 \\
\hline $64 \times 64$ & $6.71 E-02$ & 2.05 & $6.69 E-02$ & 2.05 & $6.70 E-02$ & 2.74 & $6.65 E-02$ & 2.04 & $6.60 E-02$ & 2.04 \\
\hline $128 \times 128$ & $1.44 E-02$ & 2.22 & $1.44 E-02$ & 2.22 & $1.43 E-02$ & 2.22 & $1.43 E-02$ & 2.21 & $1.43 E-02$ & 2.20 \\
\hline $256 \times 256$ & $2.39 E-03$ & 2.60 & $2.39 E-03$ & 2.59 & $2.39 E-03$ & 2.59 & $2.39 E-03$ & 2.58 & $2.39 E-03$ & 2.57 \\
\hline
\end{tabular}




\section{Capítulo}

\section{Simulação de escoamentos incompressíveis}

Neste capítulo são apresentados resultados numéricos para escoamentos incompressíveis (laminares e turbulentos) envolvendo superfícies livres móveis; ambos fluidos newtonianos e não newtonianos (viscoelástico) são considerados no estudo. Muitos dos resultados obtidos são confrontados com dados teóricos, numéricos e experimentais da literatura especializada. Para a simulação computacional foi utilizado o código Freeflow de Castelo et al. (2000) (nas versões 2D, com simetria radial e 3D) equipado com o esquema TDPUS-C3 para aproximar os termos não lineares (4.48)-(4.52) e (4.72)-(4.77).

\subsection{Resultados para fluidos newtonianos}

Esta seção é dedicada a avaliação do desempenho do esquema TDPUS-C3 em escoamentos laminares 2D e 3D. Para tanto, os seguintes problemas foram considerados: jatos livres (2D e com simetria radial) sobre uma superfície rígida impermeável, colapso de um bloco de fluido e jatos 3D (circular e planar) oscilantes.

\subsubsection{Jatos livres em regime laminar}

Quando um jato livre incide sobre uma superfície rígida impermeável, o fluido espalha em uma fina camada até que ocorre o fenômeno do súbito aumento em profundidade, conhecido como salto hidráulico (uma descontinuidade no movimento do fluido). O fenômeno pode ser observado no dia-a-dia, como por exemplo um jato de água incidente sobre a cuba da pia. A Fig. 6.1 (a) mostra o salto hidráulico circular tridimensional real e a Fig. 6.1 (b) ilustra o fenômeno no caso bidimensional. 


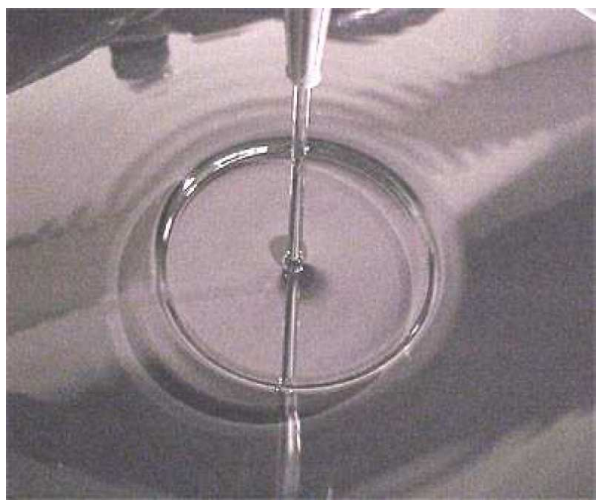

(a)

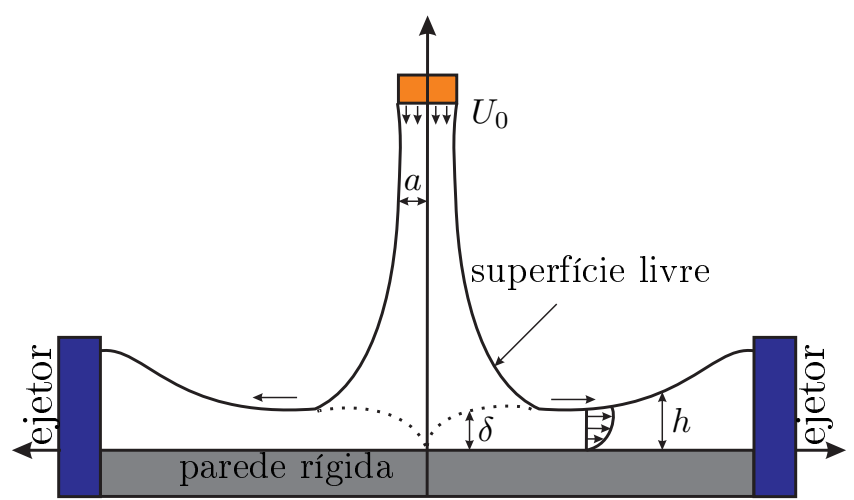

(b)

Figura 6.1: Jato de fluido incidente sobre uma superfície rigida tomado de Rai et al. (2008) (à esquerda); ilustração esquemática (à direita).

O salto hidráulico tem no caso 2D uma solução analítica para a camada (ou altura) $h$ do fluido sobre o contorno rígido a qual é dada por Watson (1964) como

$$
h(x)= \begin{cases}\frac{\pi}{\sqrt{3}} \frac{\nu(x+l)}{Q}, & x \geq x_{0} \\ a+\left(\frac{1-2 \pi}{3 \sqrt{3} c^{2}}\right) \delta(x), & x<x_{0}\end{cases}
$$

onde $a=L_{0} / 2$ é o raio do injetor e $Q=\pi a^{2} U_{0}$ é a vazão. Os parâmetros $x_{0}, l$ e $\delta(x)$ são dados por

$$
\begin{aligned}
x_{0} & =\left(\frac{3 \sqrt{3} c(\pi-c) \sqrt{3}}{2 \pi^{2}}\right) a R e \\
l & =\left(\frac{3 \sqrt{3} c(2 \sqrt{3} c-\pi)}{2 \pi^{2}}\right) a R e \\
\delta^{2}(x) & =\left(\frac{3 \sqrt{3} c^{3}}{2(\pi-c \sqrt{3})} \frac{\nu x}{U_{0}}\right)
\end{aligned}
$$

onde $c$ é uma constante que assume o valor de 1.402 (ver Watson (1964)). Para a simulação computacional do salto hidráulico no caso 2D foram consideradas as seguintes condições de contorno: na entrada perfil reto $\left(U_{0}\right)$ e na parede não escorregamento. Nas regiões de saída do fluido adotam-se as condições homogêneas de Neumann. As condições de contorno na superfície livre são dadas pelas Eqs. (4.120) onde o tensor $\sigma$ é dado por (4.3). Os dados empregados na simulação estão apresentados na Tab. 6.1.

Na Fig. 6.2 é apresentada uma comparação entre a solução analítica e a solução numérica nas malhas I, II, III e IV obtidas com o uso do esquema TDPUS-C3. Vê-se claramente por essa figura (incluindo uma ampliação na região $[0.0075,0.0175]$ ) que as soluções numéricas nas malhas mais finas (III e IV) são praticamente idênticas e essas, quando comparadas às soluções nas malhas grossas I e II, estão mais próximas da solução analítica. Vê-se também que houve convergência da solução numérica para uma solução muito próxima da solução analítica de Watson. Como ilustração as Figs. 
Tabela 6.1: Dados da simulação do jato livre 2D no regime laminar.

- Domínio: $0.1 \mathrm{~m} \times 0.025 \mathrm{~m}$;

- Raio do injetor: $a=0.002 m$;

- Malha I : $100 \times 25$ células computacionais;

- Malha II : $200 \times 50$ células computacionais;

- Malha III : $400 \times 100$ células computacionais;

- Malha IV : $800 \times 200$ células computacionais;

- Altura do injetor até a superfície rigida: $H=0.019 m$;

- Escala de comprimento: $L_{0}=0.004 m$;

- Escala de velocidade (velocidade de injeção): $U_{0}=1 \mathrm{~ms}^{-1}$;

- Coeficiente de viscosidade cinemática: $\nu=0.000002 \mathrm{~m}^{2} \mathrm{~s}^{-1}$;

- Número de Froude: $F r \approx 5.05$;

- Número de Reynolds: $R e=2000$.

6.3-6.5 mostram, na malha IV, a evolução da superfície livre do fluido no tempo e os contornos dos componentes de velocidade $u$ e $v$, e o contorno da pressão $p$. Em particular, pelo contorno da pressão mostrado na Fig. 6.5, observa-se que de fato a pressão maior ocorreu no ponto de impacto.

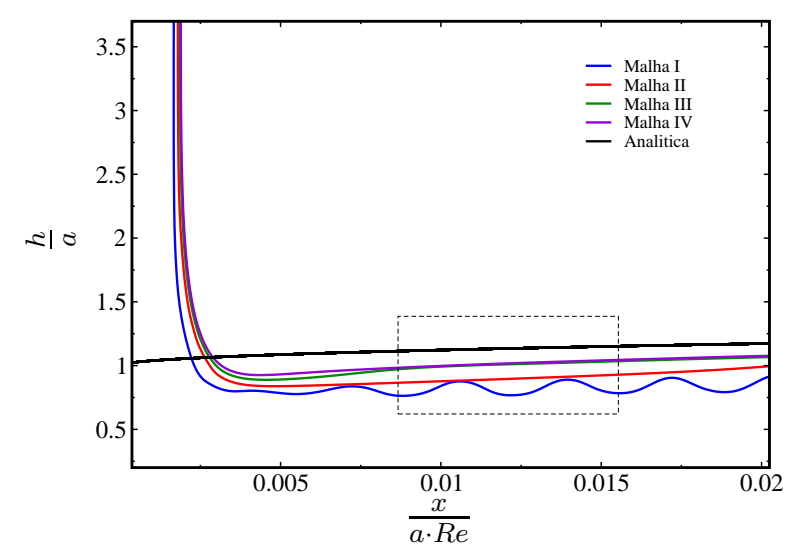

(a)

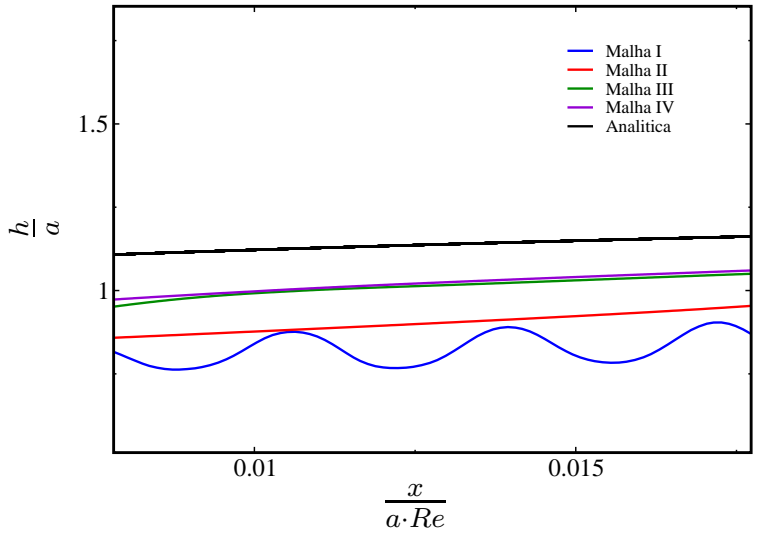

(b)

Figura 6.2: Comparação das soluções numéricas em diferentes malhas para o problema do jato livre 2D sobre uma superfície rígida no regime laminar (a); ampliação da região destacada (b). 

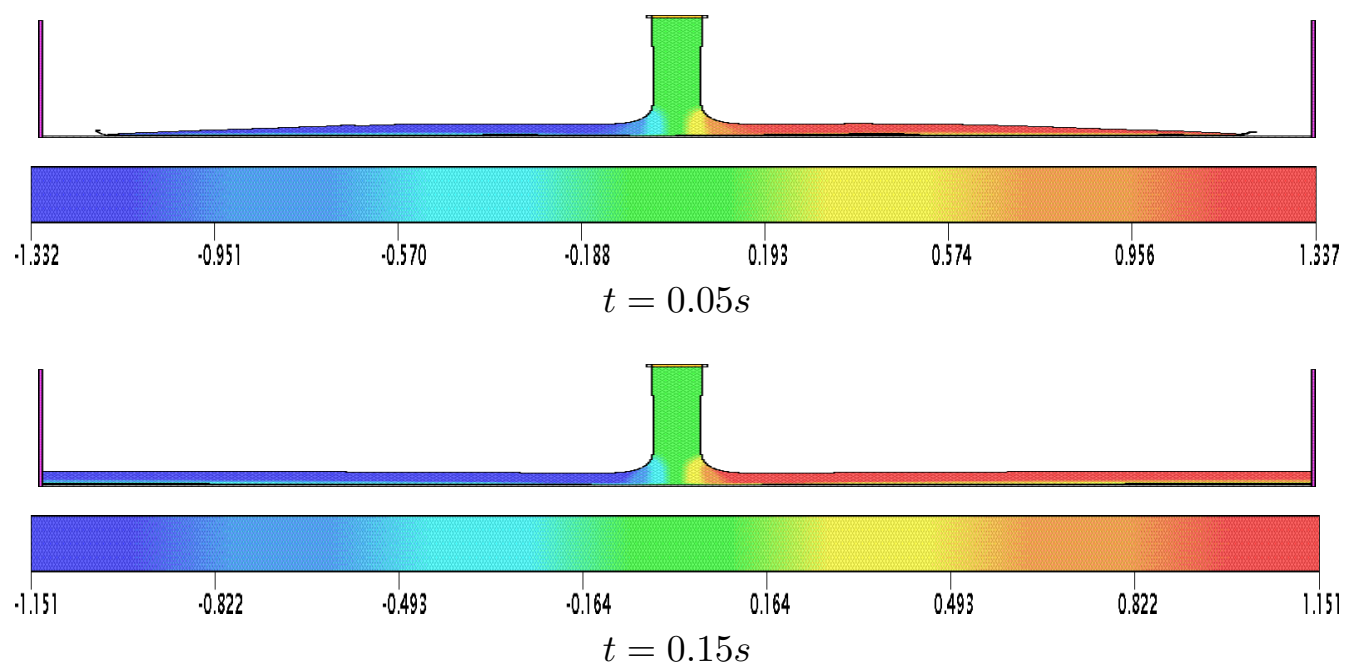

Figura 6.3: Contorno da velocidade $u$ para o problema do jato livre 2D sobre uma superfície rígida.

O salto hidráulico circular foi simulado também em uma geometria cilíndrica como ilustrado na Fig. 6.1 (a). Nas simulações, inicia-se verificando que o esquema TDPUS-C3 fornece aceitáveis estimativas para a posição do salto. As relações analíticas para o raio $R$ do salto

$$
R=\left(\frac{27 g^{-1 / 4}}{2^{1 / 4} 35 \pi}\right) Q^{2 / 3} d^{-1 / 6} \nu^{-1 / 3}
$$

de Brechet e Néda (1999) e de Bohr et al. (1993) dada por

$$
R=Q^{5 / 8} \nu^{-3 / 8} g^{-1 / 8}
$$

foram usadas para comparações. O parâmetro $d$ na Eq. (6.5) é a distância entre o injetor e a superfície rígida e assumiu nas simulações o valor de $0.03 \mathrm{~m}$. As condições de contorno adotadas neste caso foram: na entrada perfil reto $\left(U_{0}\right)$, no eixo de simetria escorregamento e na parede não escorregamento. Os dados empregados na simulação estão apresentados na Tab. 6.2.

Tabela 6.2: Dados da simulação do jato livre 2D no regime laminar em simetria radial.

- Malha I : $200 \times 126$ células computacionais;

- Malha II : $400 \times 252$ células computacionais;

- Malha III : $800 \times 504$ células computacionais;

- Raio do injetor: $a=0.004 m$;

- Domínio: $0.05 \mathrm{~m} \times 0.0315 \mathrm{~m}$;

- Altura do injetor: $H=0.004 m$;

- Escala de comprimento: $L_{0}=0.008 m$;

- Escala de velocidade (velocidade de injeção): $U_{0}=0.375 \mathrm{~ms}^{-1}$;

- Coeficiente de viscosidade cinemática: $\nu=0.000012 \mathrm{~m}^{2} \mathrm{~s}^{-1}$;

- Número de Froude: $\mathrm{Fr}=1.3386$;

- Número de Reynolds: $R e=250$;

A Tab. 6.3 mostra as estimativas numéricas do raio do salto hidráulico $r$ obtido nas simulações 


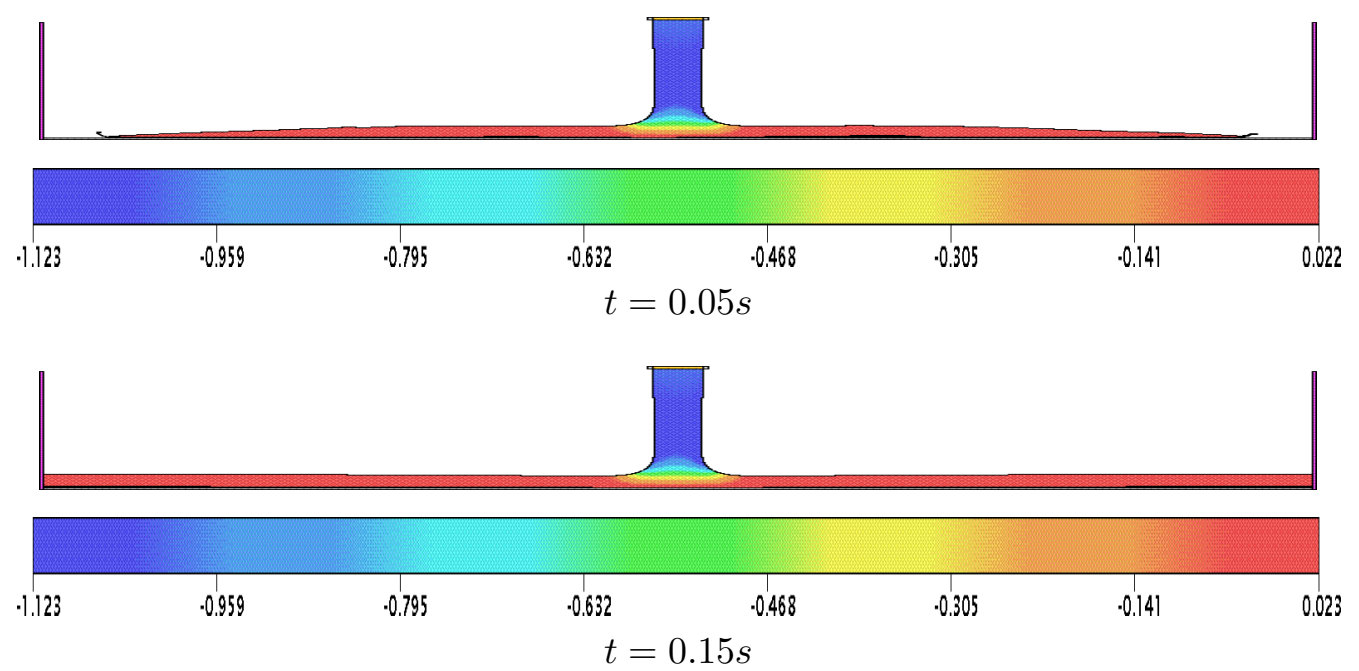

Figura 6.4: Contorno da velocidade $v$ para o problema de jato livre 2D sobre uma superfície rígida no regime laminar.
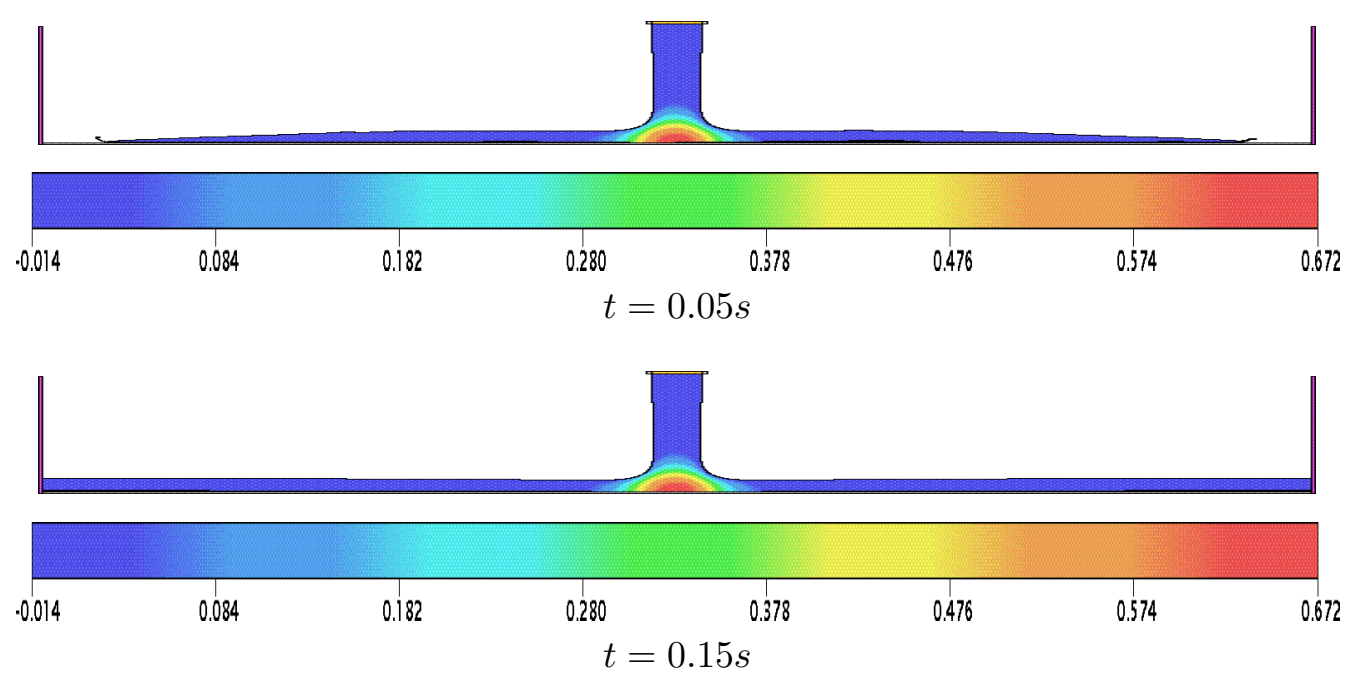

Figura 6.5: Contorno da pressão $p$ para o problema do jato livre 2D sobre uma superfície rígida. 
numéricas com o esquema TDPUS-C3 e os dados teóricos de Brechet e Néda e Bohr et al.. Observa-se

Tabela 6.3: Estimativas teóricas e numéricas nas malhas I, II e III para o raio do salto hidráulico.

\begin{tabular}{|c|c|c|}
\hline Raio teórico de Brechet e Néda (1999) & Estimativas por TDPUS-C3 & Raio teórico de Bohr et al. (1993) \\
\hline & Malha I Malha II Malha III & \\
\hline 0.013 & 0.028 & 0.059 \\
\hline
\end{tabular}

por essa tabela que, com o código Freeflow equipado com o esquema TDPUS-C3, os valores numéricos obtidos para o raio do salto estão entre o limitante inferior devido a Brechet e Néda (1999) e o limitante superior devido a Bohr et al. (1993); e mais uma estimativa para esse comprimento é $\approx$ 0.025, concordando razoavelmente bem com o dado teórico de Brechet e Néda, o qual foi verificado anteriormente por Hansen et al. (1997).

Uma comparação foi feita também entre a camada do fluido $h$, calculada com o esquema TDPUS-C3 nas malhas I, II e III e a solução viscosa de Watson (ver Fig. 6.6). A análise foi feita na região $0.2<(r / a) R e^{1 / 3}<0.8$ uma vez que essa análise é válida para $r / a \gg 1$ e a presença do contorno de saída de fluido. Observa-se claramente nessa figura que as soluções numéricas são não oscilatórias, e quando a malha é refinada a solução converge, indicando a convergência do método numérico global para esse escoamento (complexo) com superfície livre. A solução analítica de Watson é válida somente num intervalo restrito de $r$ e os resultados apresentados na Fig. 6.6 são muito bons neste intervalo.

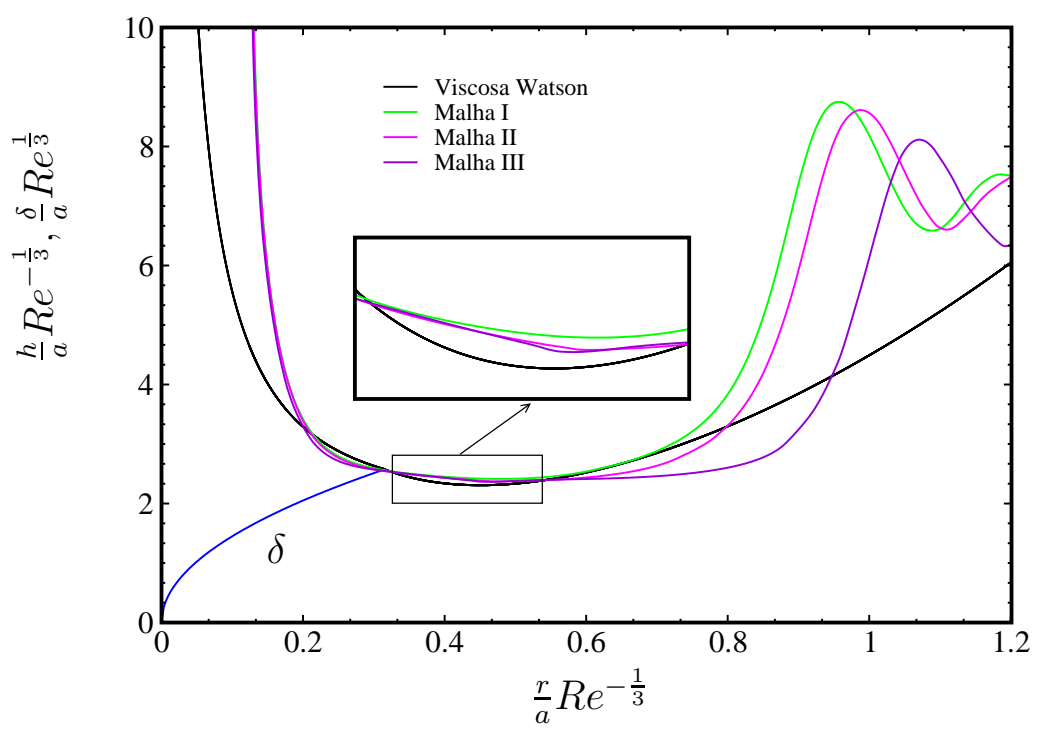

Figura 6.6: Comparação entre as soluções numéricas da altura $h$ obtidas com TDPUS-C3 e analítica para o problema de simetria radial. 
Na Fig. 6.7 apresentam-se, no tempo $t=1.5 \mathrm{~s}$ visualizações, tridimensionais da simulação numérica, na malha mais grossa, do salto hidráulico circular obtida com o esquema TDPUS-C3. Observa-se a partir dessas simulações que o esquema TDPUS-C3 conseguiu capturar qualitativamente bem o fenômeno físico salto hidráulico circular e as ondas de superfície nas regiões além da descontinuidade, ambos mostrado no experimento de Rai et al. (2008) na Fig. 6.1 (a).
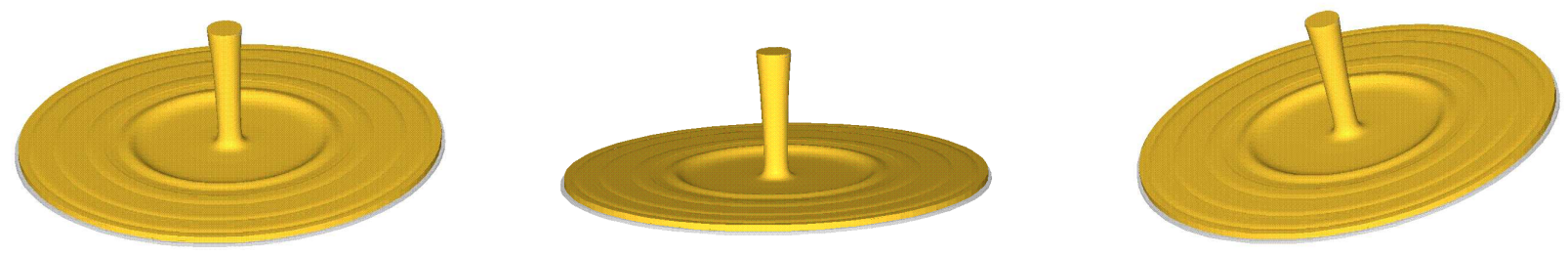

Figura 6.7: Ressalto hidráulico circular em distintos ângulos simulado pelo esquema TDPUS-C3. $R e=250, t=1.5 s$ e a malha de $200 \times 126$.

\subsubsection{Colapso de um bloco de fluido em regime laminar}

O problema do colapso de um bloco fluido (ou ruptura da barragem) consiste numa coluna de fluido em equilíbrio hidrostático que sob à ação da gravidade colapsa (ver Fig.6.8). O fenômeno foi estudado experimentalmente por Martin e Moyce (1952), e tem sido usado frequentemente como um benchmark teste para validar esquemas numéricos para escoamentos com superfícies livres móveis (ver, por exemplo, Kim e Leer (2003), Murrone e Guillard (2008), Nikitin et al. (2011) e Cruchaga et al. (2007)).

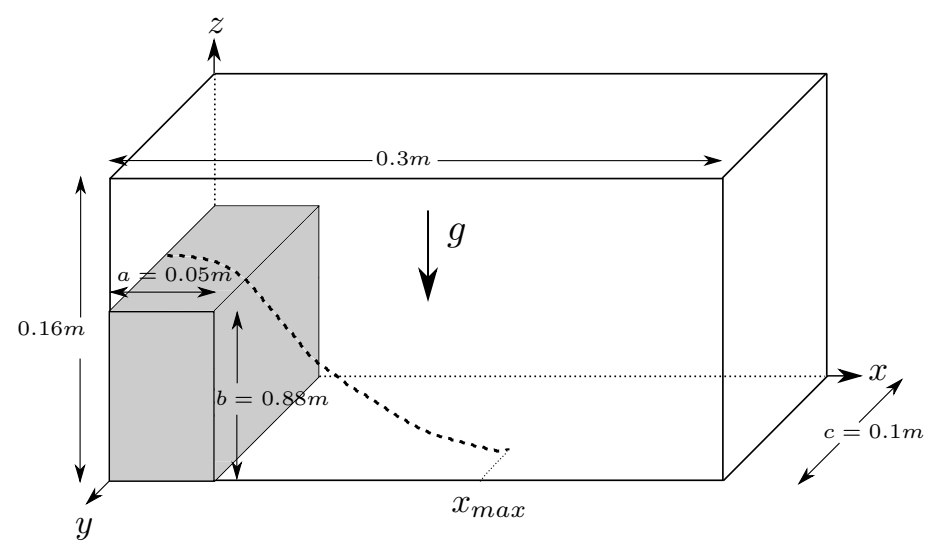

Figura 6.8: Ilustração esquemática do colapso de um bloco de fluido.

Apresenta-se a seguir simulação computacional do problema em regime laminar do colapso utilizando o ambiente Freeflow equipado com o esquema TDPUS-C3. Para tanto, considerou-se um bloco de fluido de dimensões $a=0.05 m, b=0.88 m$ e $c=0.1 m$ (ver Fig. 6.8), cujas paredes são aplicadas o deslizamento como condição de contorno. Os dados adotados nos cálculos estão apresentados na Tab. 6.4. 
Tabela 6.4: Dados da simulação do colapso de um bloco de fluido.

- Malha : $150 \times 50 \times 80$ células computacionais;

- Domínio: $0.3 m \times 0.1 m \times 0.16 m$;

- Escala de comprimento: $L_{0}=0.1 \mathrm{~m}$;

- Escala de velocidade: $U_{0}=\sqrt{g L_{0}}=0.9905 \mathrm{~ms}^{-1}$;

- Coeficiente de viscosidade cinemática: $\nu=0.000001 \mathrm{~m}^{2} \mathrm{~s}^{-1}$;

- Número de Froude: $F r \approx 1.0$;

- Número de Reynolds: $R e \approx 99045$.

Os resultados numéricos extraídos no plano $x z$ (na posição $y=0.05$ ) da evolução do espalhamento horizontal $x_{\max } / a$ contra $t \sqrt{2 g / a}$ (ver Fig. 6.8) obtidos com TDPUS-C3 estão apresentados na Fig. 6.9. Essa mesma figura contêm os dados numéricos obtidos com o esquema TOPUS de Ferreira et al. (2012), com os métodos BEM, Level Set e SPH, com a solução teórica de Ritter (1982), e dados experimentais de Martin e Moyce (1952) (ver Colagrossi e Landrini (2003)). Vê-se por essa figura que os dados numéricos obtidos com TDPUS-C3 estão em boa concordância com àqueles mencionados anteriormente. A evolução da superfície livre do fluido bem como os contornos de $u, w$ e $p$ nos tempos $t=0.05 s, 0.1 s, 0.15 s$ e $0.2 s$ estão mostrados nas Figs. 6.10-6.12.

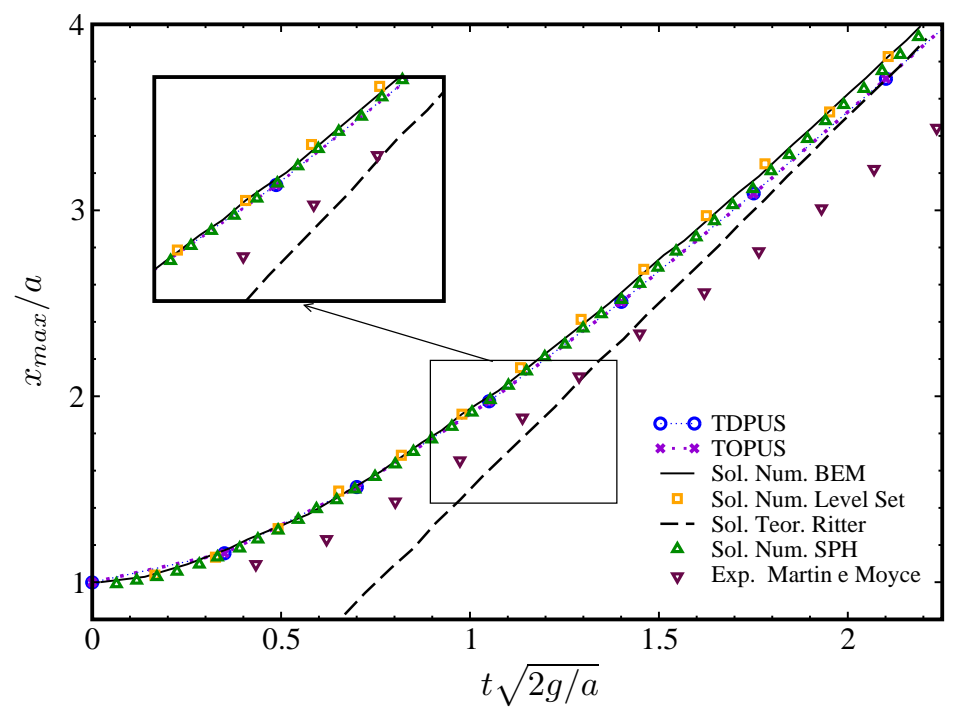

Figura 6.9: Soluções numéricas para o espalhamento horizontal, $x_{\max }$, obtidas com TDPUS-C3 para o problema do colapso de um bloco de fluido 3D no regime laminar. 
$t=0.05 s$

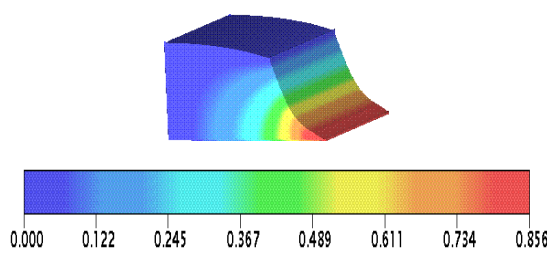

$t=0.15 s$

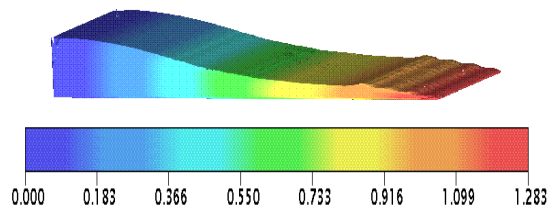

$$
t=0.1 s
$$

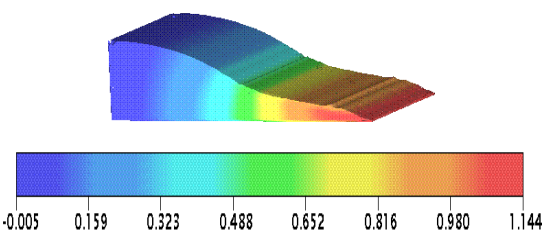

$t=0.2 s$

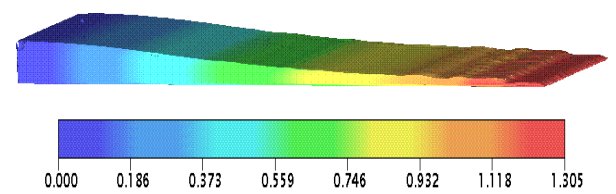

Figura 6.10: Contorno da velocidade $u$ para o problema do colapso de um bloco de fluido 3D no regime laminar com TDPUS-C3.

$t=0.05 s$

$$
t=0.1 s
$$

$$
t=0.2 s
$$
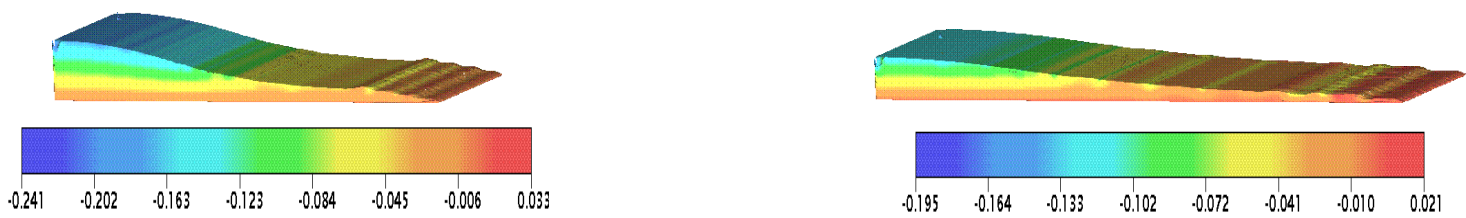

Figura 6.11: Contorno da velocidade $w$ para o problema do colapso de um bloco de fluido 3D no regime laminar com TDPUS-C3. 
$t=0.05 s$

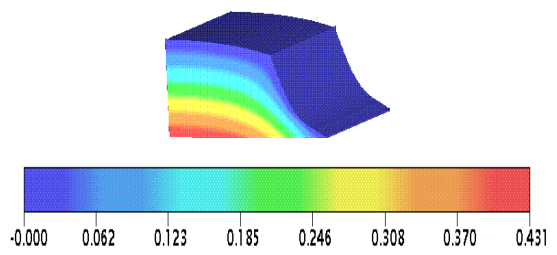

$t=0.15 s$

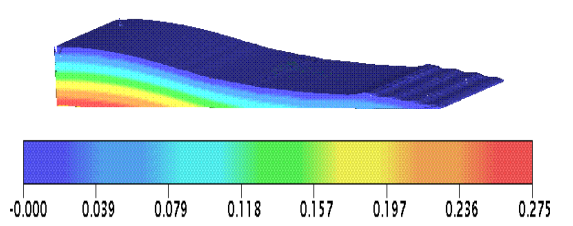

$$
t=0.1 s
$$

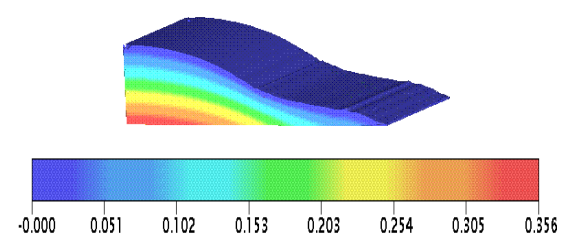

$t=0.2 s$

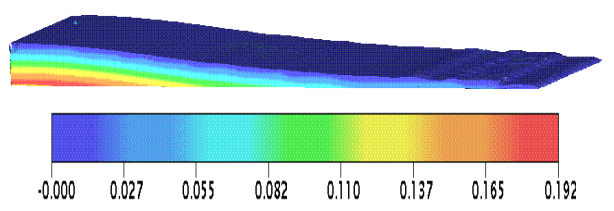

Figura 6.12: Contorno da pressão $p$ para o problema do colapso de um bloco de fluido 3D no regime laminar com TDPUS-C3.

\subsubsection{Jatos livres 3D a baixos valores do número de Reynolds}

As simulações tridimensionais apresentadas a seguir têm como propósito mostrar que, muito embora o esquema TDPUS-C3 tenha sido planejado para problemas a altos valores do número de Reynolds, ele pode simular com sucesso escoamentos a baixos valores do número de Reynolds. O problema escolhido foi o de um jato (cilíndrico ou planar) altamente viscoso incidindo sobre uma superfície rígida impermeável a uma altura $H$, em que sob certas condições o jato ao atingir a superfície rígida apresenta o fenômeno (físico) de dobras. Esse fenômeno está ilustrado na Fig. 6.13. Desprezando os efeitos da tensão superficial, há cinco trabalhos principais, isto é Cruickshank (1980), Cruickshank e Munson (1981, 1982), Cruickshank (1988) e Mahadevan et al. (1998), onde os autores mostram via métodos teóricos e experimentais que o fenômeno de dobras em jatos circulares de fluidos viscosos surge devido a dois valores críticos: (i) a razão de aspecto $H / d$ que deve ser maior que um valor de razão de aspecto crítico $(H / d)_{c}=7$, onde $d$ o diâmetro do injetor; e (ii) o numero de Reynolds que deve ser menor que o valor crítico $R e_{c}=1.2$. Fazendo o uso do ambiente Freeflow 3D equipado com o esquema TDPUS-C3, três testes foram realizados para prever o fenômeno de dobras, sendo dois deles usando diâmetros diferentes para o injetor, Teste 1 e Teste 2; e um, o Teste 3, para o caso do jato planar. A geometria empregada nos Teste 1 e Teste 2 está mostrada na Fig 6.14 (a), e a geometria para o Teste 3 está mostrada na 6.14 (b). A condição de contorno adotada nas superfícies rígidas foi a de não deslizamento em todos os testes.

Para o Teste 1 o diâmetro do injetor foi tomado como $d=0.04 m$ e a razão de aspecto crítica como $H / d=1 \mathrm{~m} / 0.04 m=25$; dados esses satisfazendo as condições de Cruickshank. Os dados adotados para este teste estão apresentados na Tab. 6.5. 

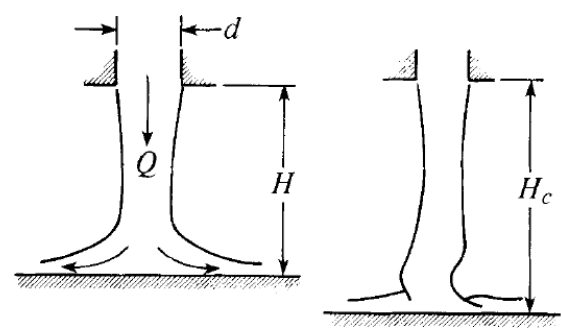

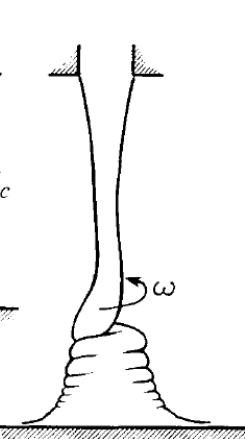

(c)

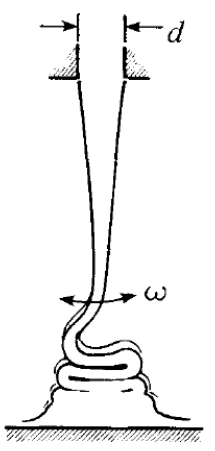

(d)

Figura 6.13: Estruturas dos jatos oscilantes viscosos segundo Cruickshank e Munson (1981). (a) Jato estável, sem oscilações; (b) jato com oscilações que surgem na altura critica; (c) jato axisimétrico oscilante; (d) jato planar oscilante. $Q$ é a vazão e $\omega$ a frequência das oscilações do jato.

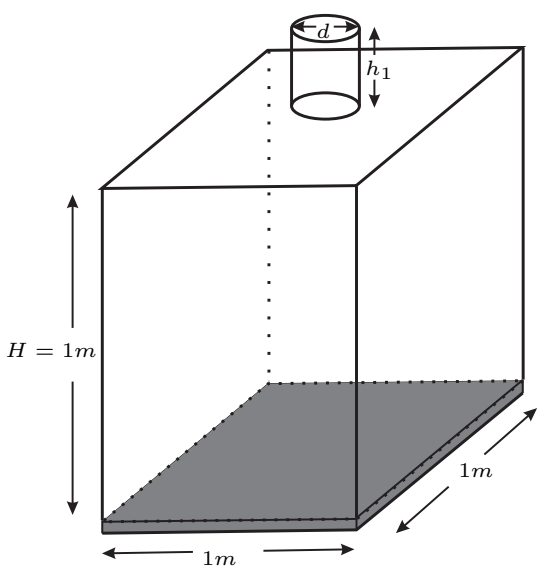

(a)

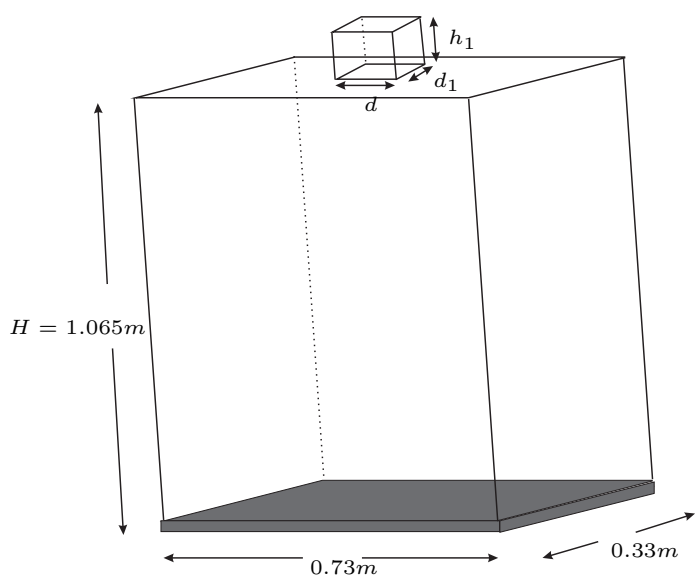

(b)

Figura 6.14: Geometria para o problema do jato circular (a) e planar (b). 
Tabela 6.5: Dados da simulação do jato circular oscilante. Teste 1.

- Malha : $100 \times 100 \times 123$ células computacionais;

- Domínio: $1 \mathrm{~m} \times 1 \mathrm{~m} \times 1.23 \mathrm{~m}$;

- Diâmetro do injetor: $d=0.04 m$;

- Altura do injetor: $h_{1}=0.2 m$;

- Escala de comprimento: $L_{0}=2 d=0.08 m$;

- Escala de velocidade: $U_{0}=1 \mathrm{~ms}^{-1}$;

- Coeficiente de viscosidade cinemática: $\nu=0.278 \mathrm{~m}^{2} \mathrm{~s}^{-1}$;

- Número de Froude: $F r \approx 1.13$;

- Número de Reynolds: $R e \approx 0.288$.

A Fig.6.15 mostra a comparação qualitativa entre a solução numérica em vários tempos e a experimental dada por Nóbrega et al. (2007). Como pode ser visto por essa comparação, a solução numérica reproduziu o fenômeno de dobras mostrado no experimento. Entretanto, vê-se claramente que o resultado numérico não correspondeu fielmente o experimento físico. Para corrigir esse defeito na simulação computacional aumentou-se o raio do injetor. O Teste 2, refletindo essa alteração, foi simulado com diâmetro do injetor tomado como $d=0.08 \mathrm{~m}$; a razão de aspecto $H / d=1 \mathrm{~m} / 0.08 \mathrm{~m}=12.5$ neste caso continua sendo maior que a razão de aspecto crítica $(H / d)_{c}$. Os dados adotados para este teste estão apresentados na Tab. 6.6.

Tabela 6.6: Dados da simulação do jato circular oscilante. Teste 2.

- Malha : $50 \times 50 \times 63$ células computacionais;

- Domínio: $1 \mathrm{~m} \times 1 \mathrm{~m} \times 1.26 \mathrm{~m}$;

- Diâmetro do injetor: $d=0.08 m$;

- Altura do injetor: $h_{1}=0.2 m$;

- Escala de comprimento: $L_{0}=d=0.08 m$;

- Escala de velocidade: $U_{0}=1 \mathrm{~ms}^{-1}$;

- Coeficiente de viscosidade cinemática: $\nu=0.278 m^{2} s^{-1}$;

- Número de Froude: $F r \approx 1.13$;

- Número de Reynolds: $R e \approx 0.288$.

A Fig. 6.16 mostra a superfície livre do fluido no Teste 2 (novo diâmetro para o injetor), onde pode-se ver que o fenômeno de dobras (e a própria simulação) está mais consistente com os experimentos de Nóbrega et al. (2007). Para simples ilustração, a Fig. 6.17 mostra no tempo $t=8 s$ os contornos dos componentes de velocidade $u$ e $v$ e o campo de pressão $p$.

Para o Teste 3, isto é para caso do jato planar ilustrado na Fig. 6.14 (b), os experimentos de Cruickshank e Munson (1981) e Cruickshank (1988) sugerem que as instabilidades físicas em escoamentos viscosos (ou o fenômeno de dobras) provavelmente ocorrerá quando $R e<0.56$ e a razão de aspecto $\left(H / d_{1}\right)_{c}>9.4247779608 \approx 3 \pi$, onde $d_{1}$ é a largura do injetor (ver Fig. 6.14 (b)). Na simulação 
apresentada a seguir, a razão de aspecto considerada foi $H / d_{1}=1.065 m / 0.02 m=53.25$ e o número de Reynolds como $R e=U_{0} d / \nu \approx 0.72$; os restantes dos dados para este teste estão apresentados na Tab. 6.7.

Tabela 6.7: Dados da simulação do jato circular oscilante. Teste 3.

- Malha : $146 \times 66 \times 213$ células computacionais;

- Domínio: $0.73 m \times 0.33 m \times 1.065 m$;

- Comprimento do injetor: $d=0.2 m$;

- Largura do injetor: $d_{1}=0.02 m$;

- Altura do injetor: $h_{1}=0.05 \mathrm{~m}$;

- Escala de comprimento: $L_{0}=d=0.2 m$;

- Escala de velocidade: $U_{0}=1 \mathrm{~ms}^{-1}$;

- Coeficiente de viscosidade cinemática: $\nu=0.278 \mathrm{~m}^{2} \mathrm{~s}^{-1}$;

- Número de Froude: $F r \approx 0.71$;

- Número de Reynolds: $R e \approx 0.72$.

A Fig. 6.18 apresenta a simulação computacional do Teste 3, mostrando a superfície livre do fluido em diferentes tempos. E como mostrado nesta figura, muito embora as condições para instabilidade de Cruickshank e Munson (1981) e Cruickshank (1988) não são (completamente) satisfeitas, a simulação tridimensional com o esquema TDPUS-C3 apresentou o fenômeno de dobras, mostrando estar em perfeita concordância com os resultados de Majmudar et al. (2010). Para simples ilustração, a Fig. 6.20 mostra os contornos dos componentes de velocidade $u, v, w$ e o campo de pressão $p$ no tempo $t=1.2 s$. 


$$
t=1.5 \mathrm{~s}
$$
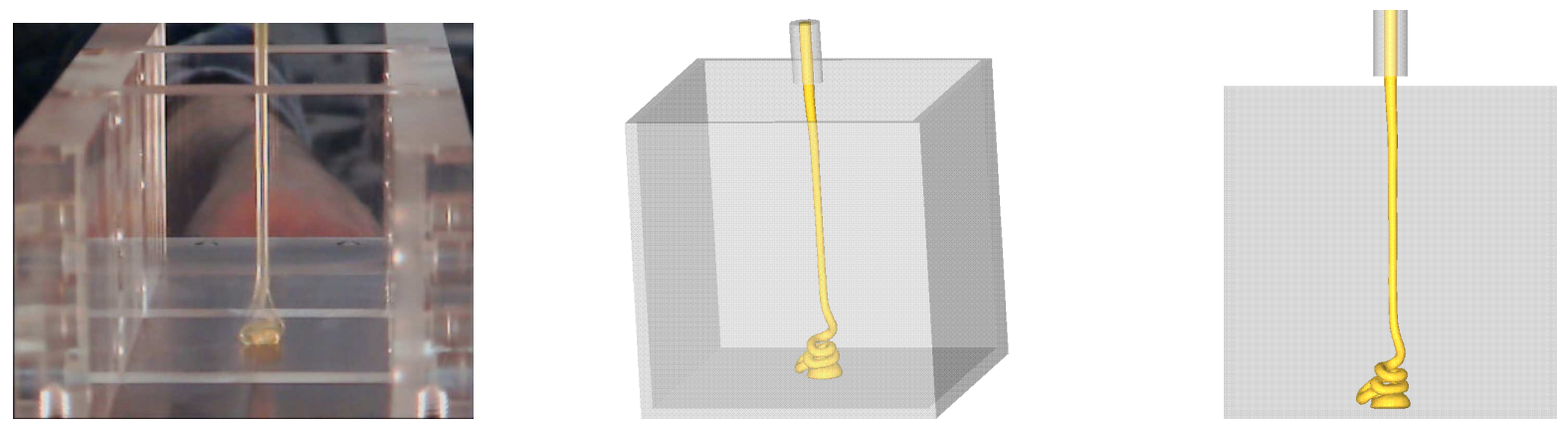

$t=2.8 s$
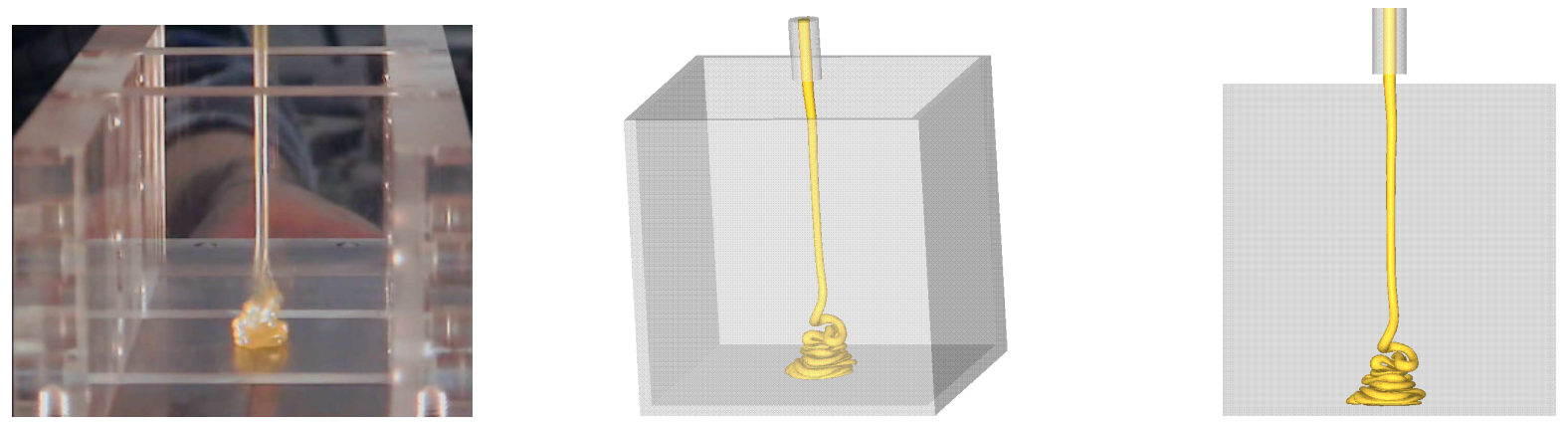

$$
t=5.1 \mathrm{~s}
$$
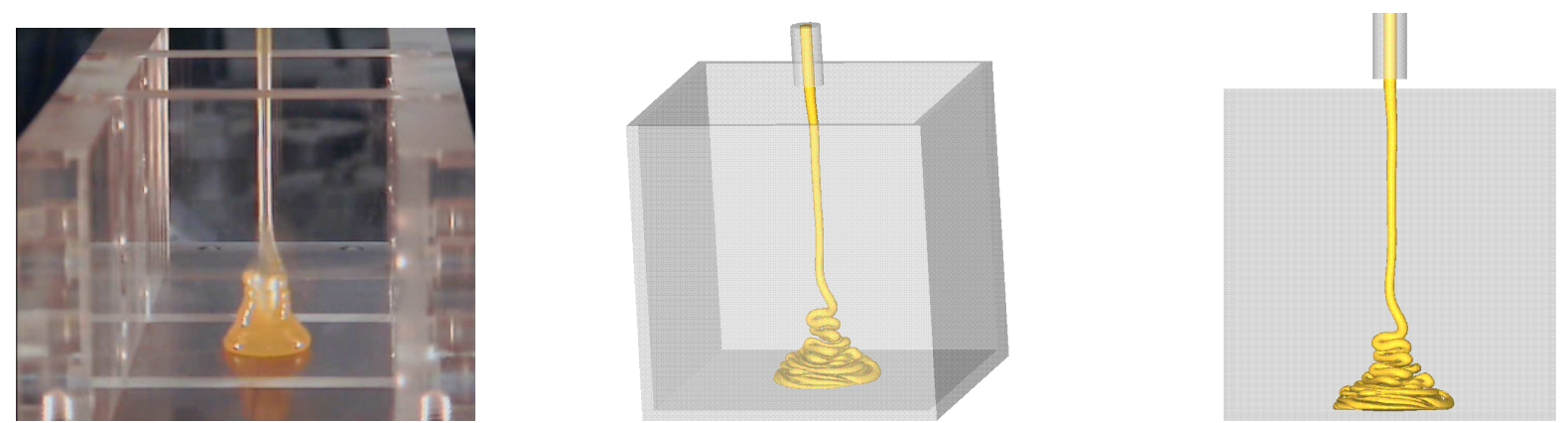

$t=9.4 s$

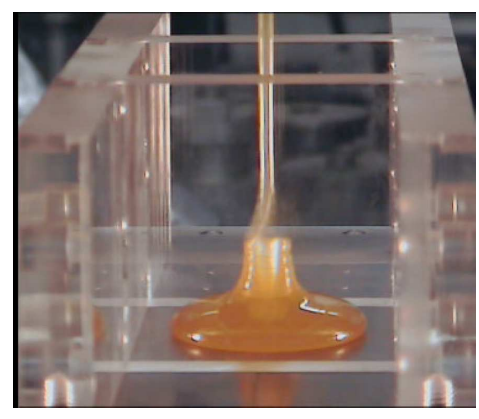

(a)

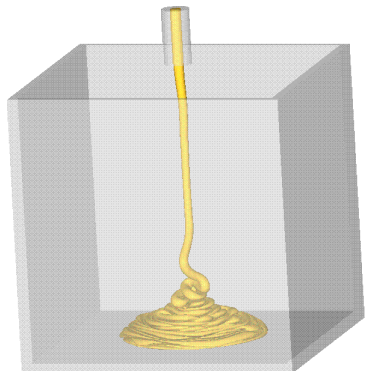

(b)

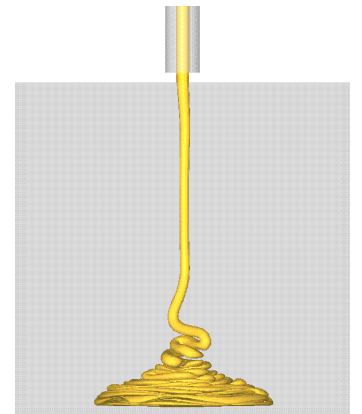

(c)

Figura 6.15: Evolução temporal do jato circular oscilante. Comparação dos dados experimentais (a) com os numéricos (b) (vista plano $x y z$ ) e (c) (vista frontal). Teste 1. 


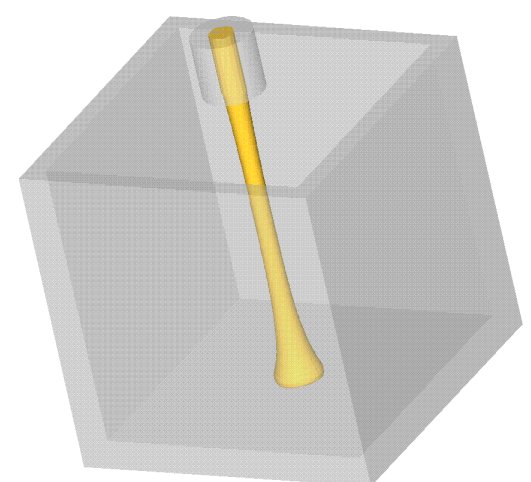

$t=1 s$

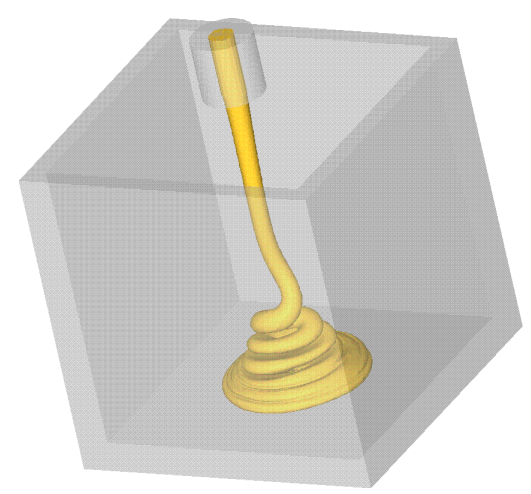

$t=4 s$

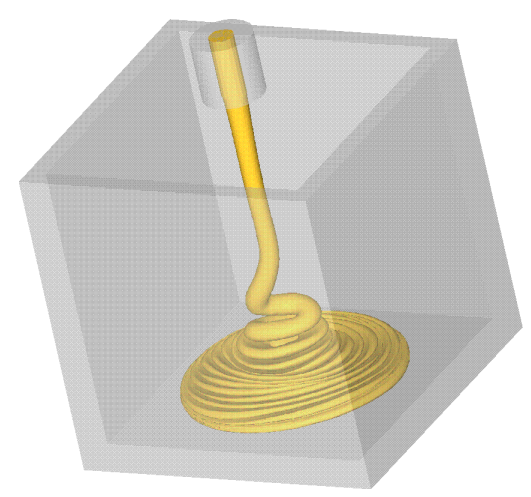

$t=7 s$

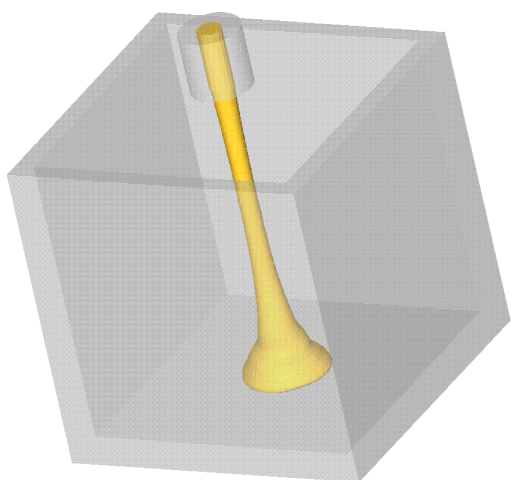

$t=2 s$

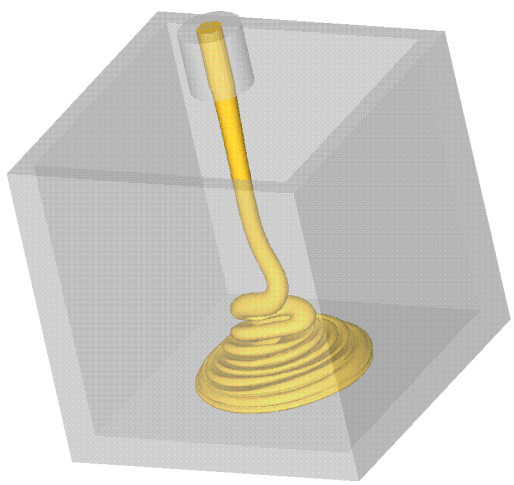

$t=5 s$

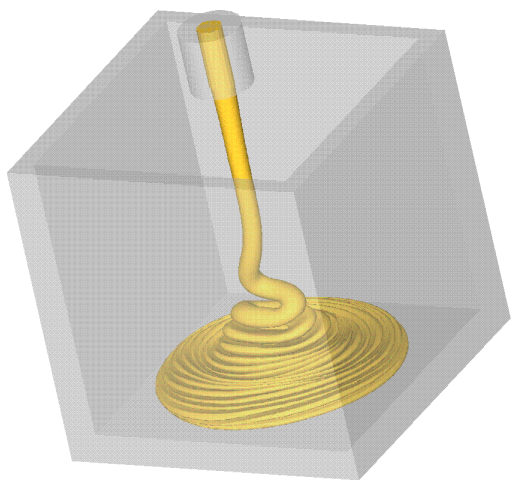

$t=8 s$

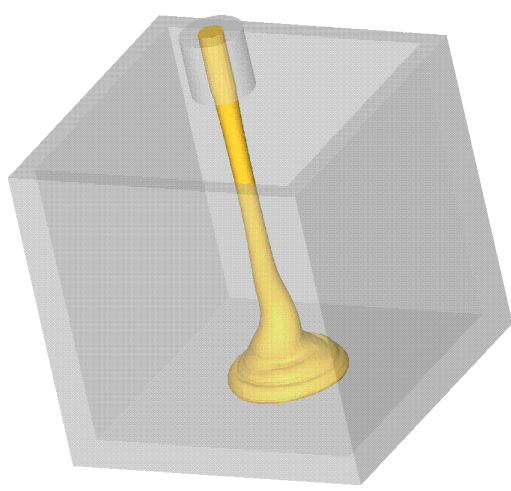

$t=3 s$

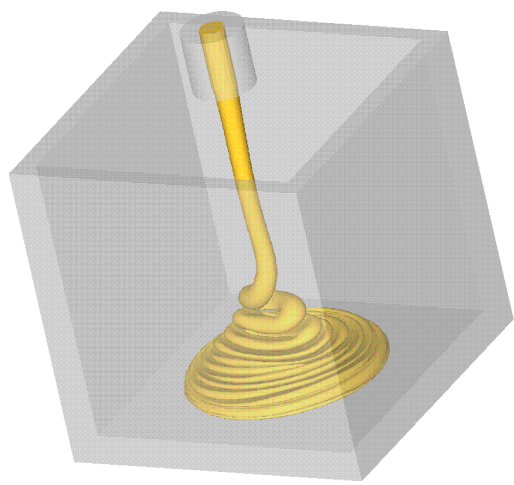

$t=6 s$

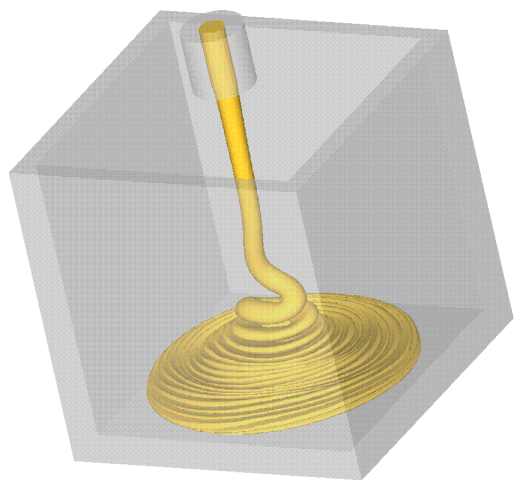

$t=9 s$

Figura 6.16: Evolução temporal do jato circular oscilante. Teste 2. 

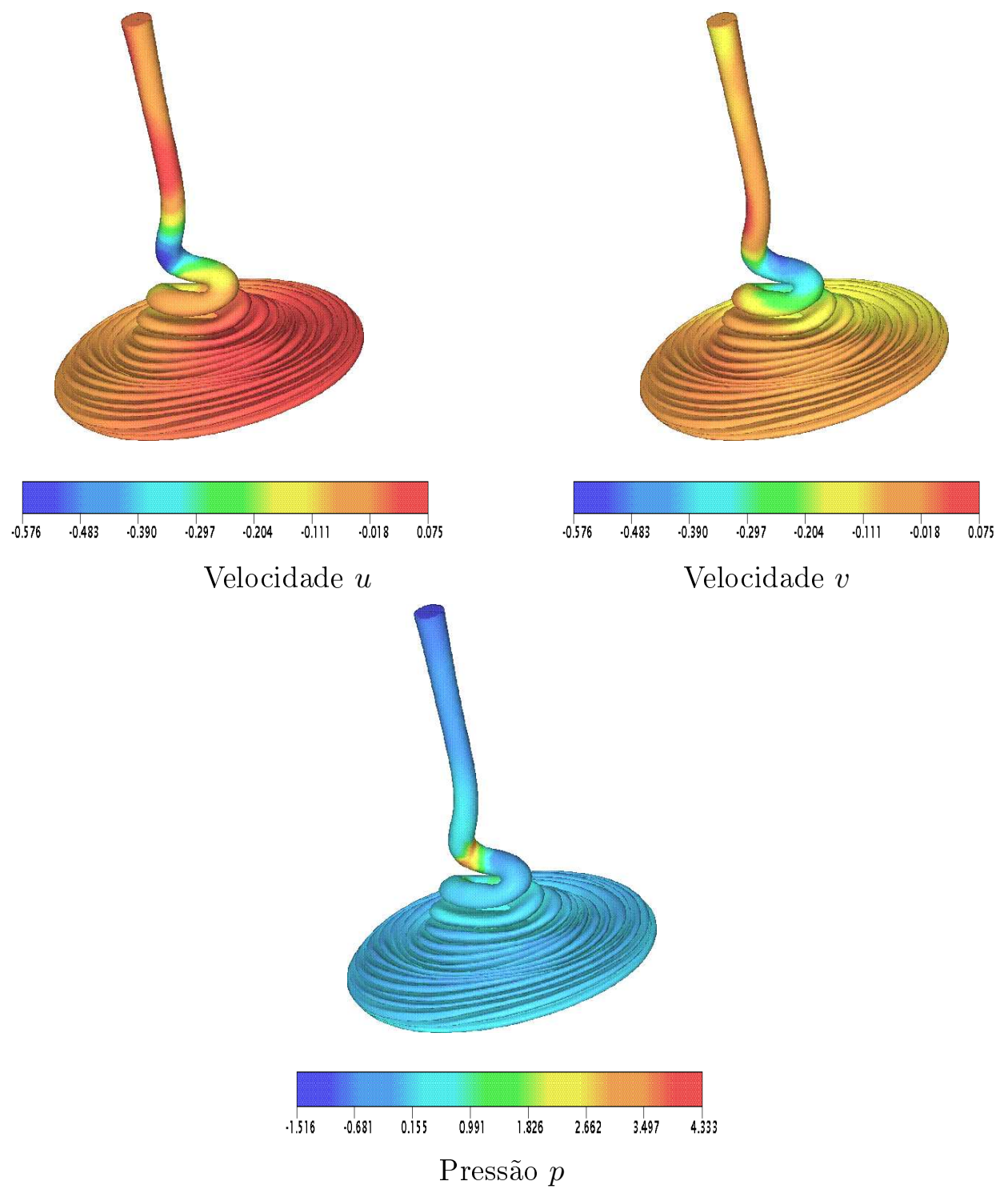

Figura 6.17: Contorno da velocidade $u, v$ e da pressão $p$ do jato circular oscilante, $t=8 s$. Teste 2 
$t=0.2 s$
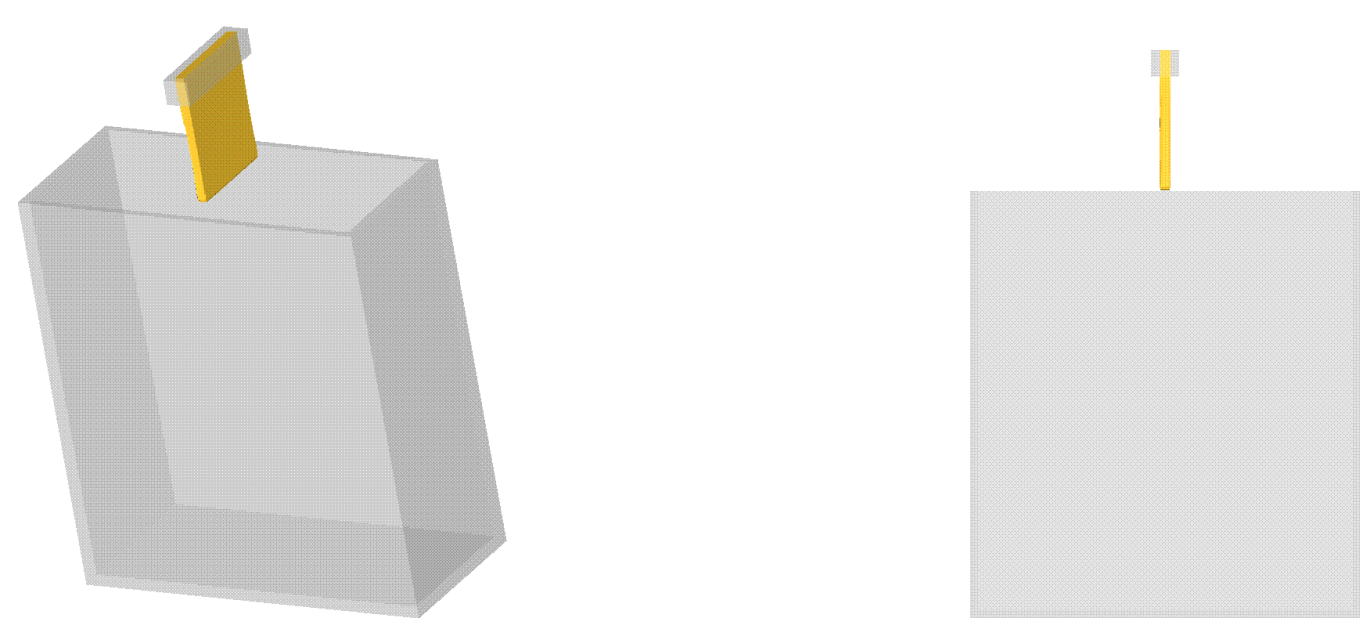

$t=0.4 s$

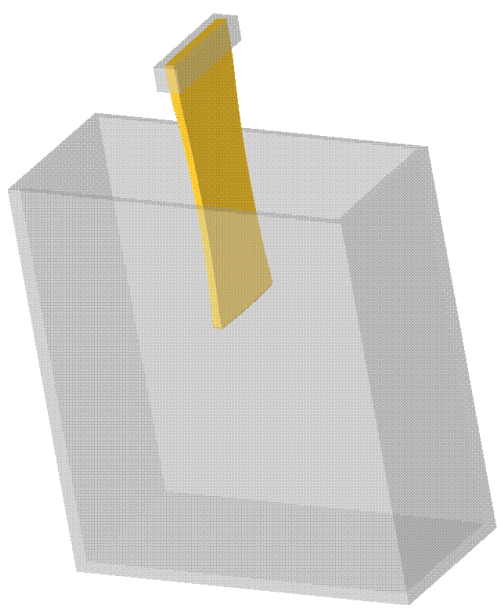

$t=0.6 s$
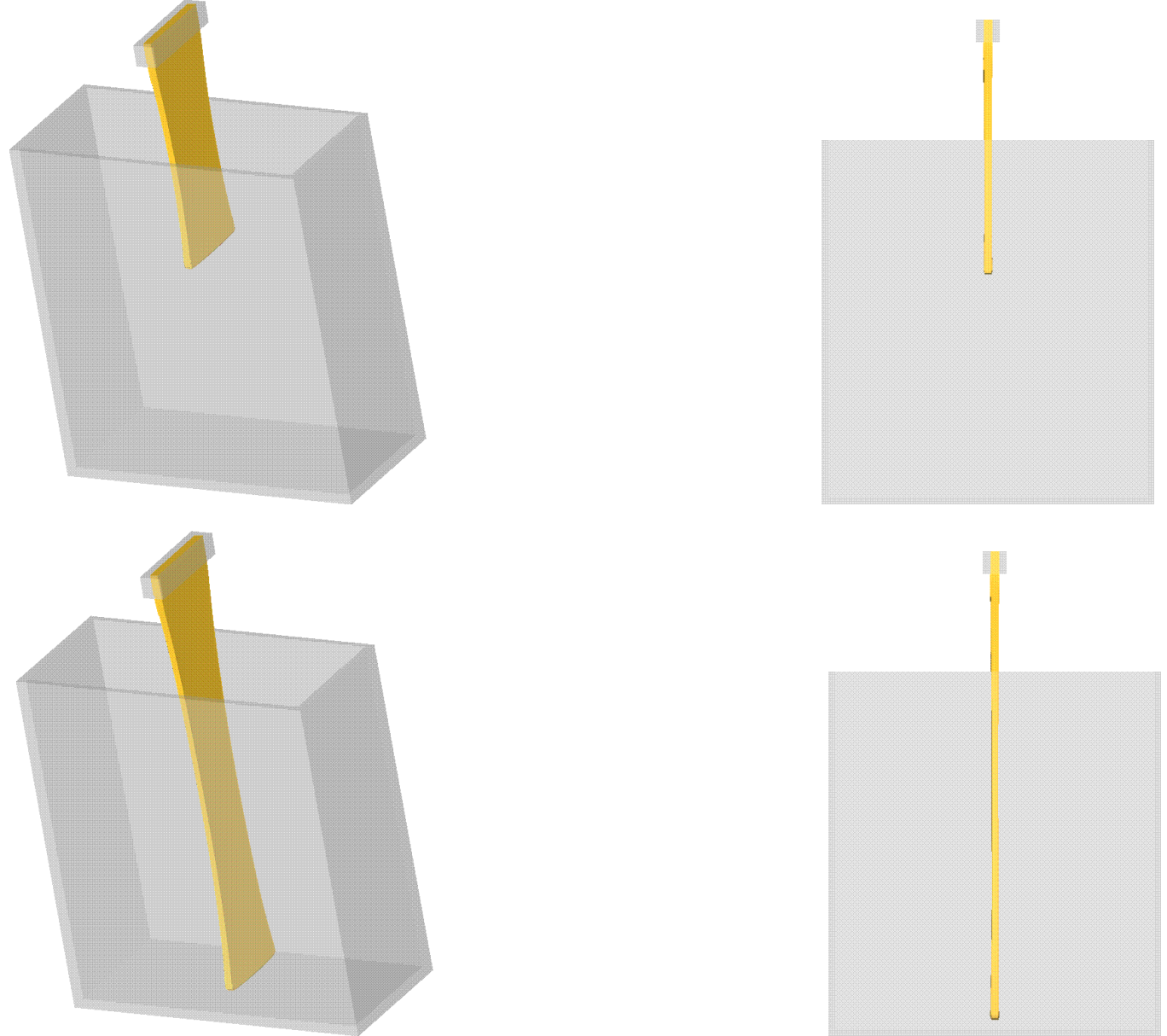

Figura 6.18: Evolução temporal do jato planar oscilante com TDPUS-C3. À esquerda superfície no plano $x y z$ e à direita vista frontal. 
$t=0.8 s$
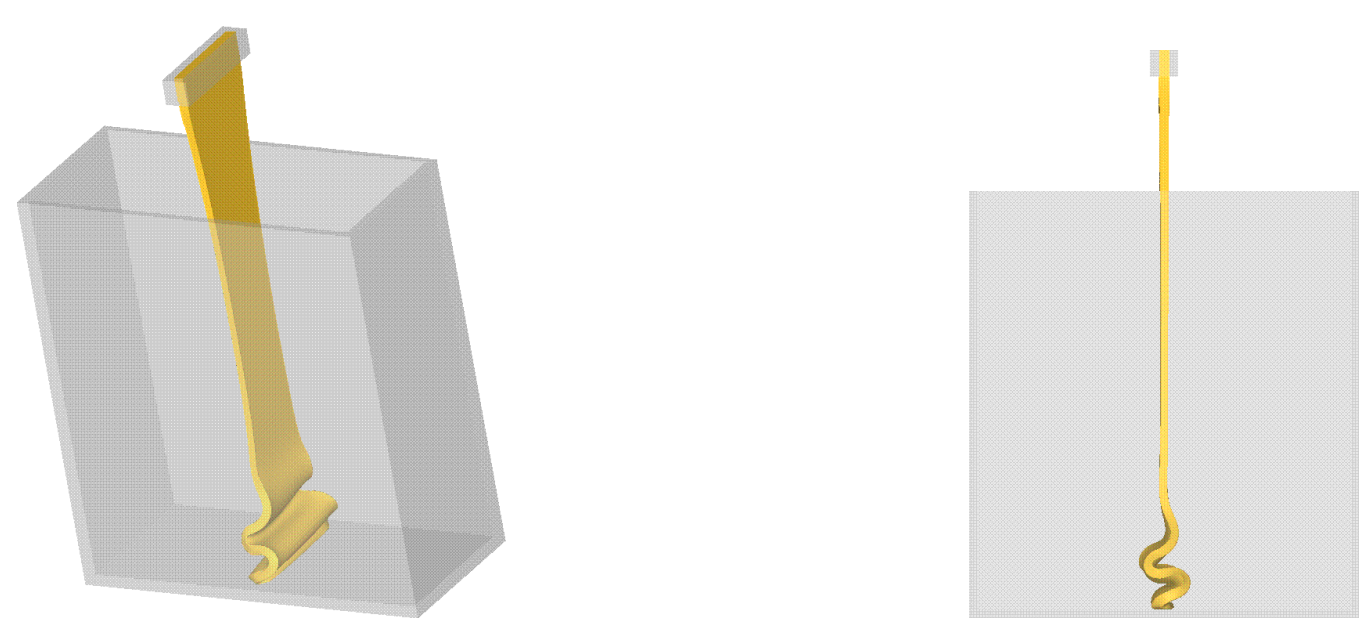

$t=1 s$
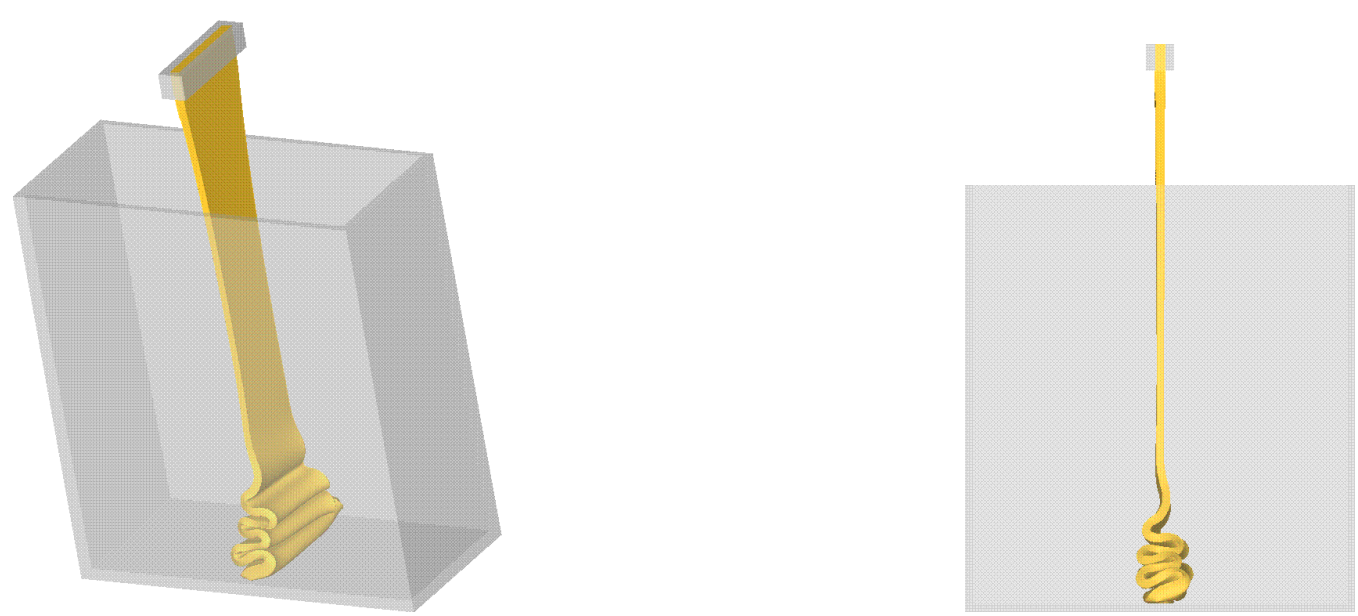

$t=1.2 \mathrm{~s}$
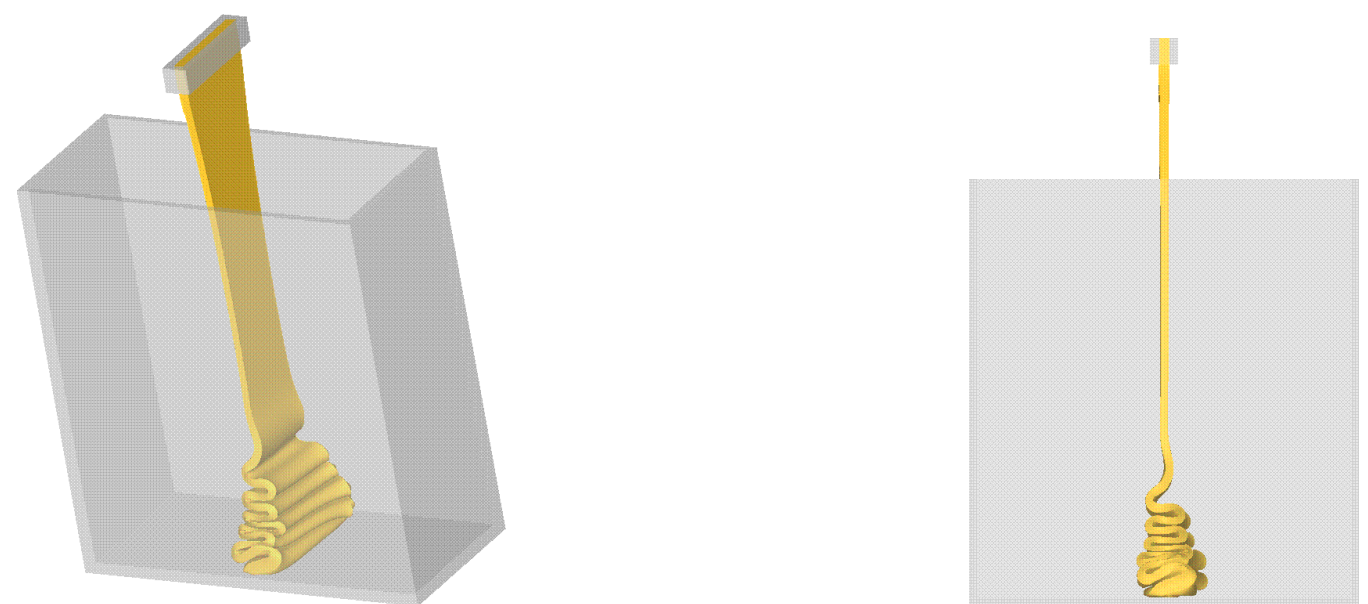

Figura 6.19: Evolução temporal do jato planar oscilante com TDPUS-C3. À esquerda superfície no plano $x y z$ e à direita vista frontal. 

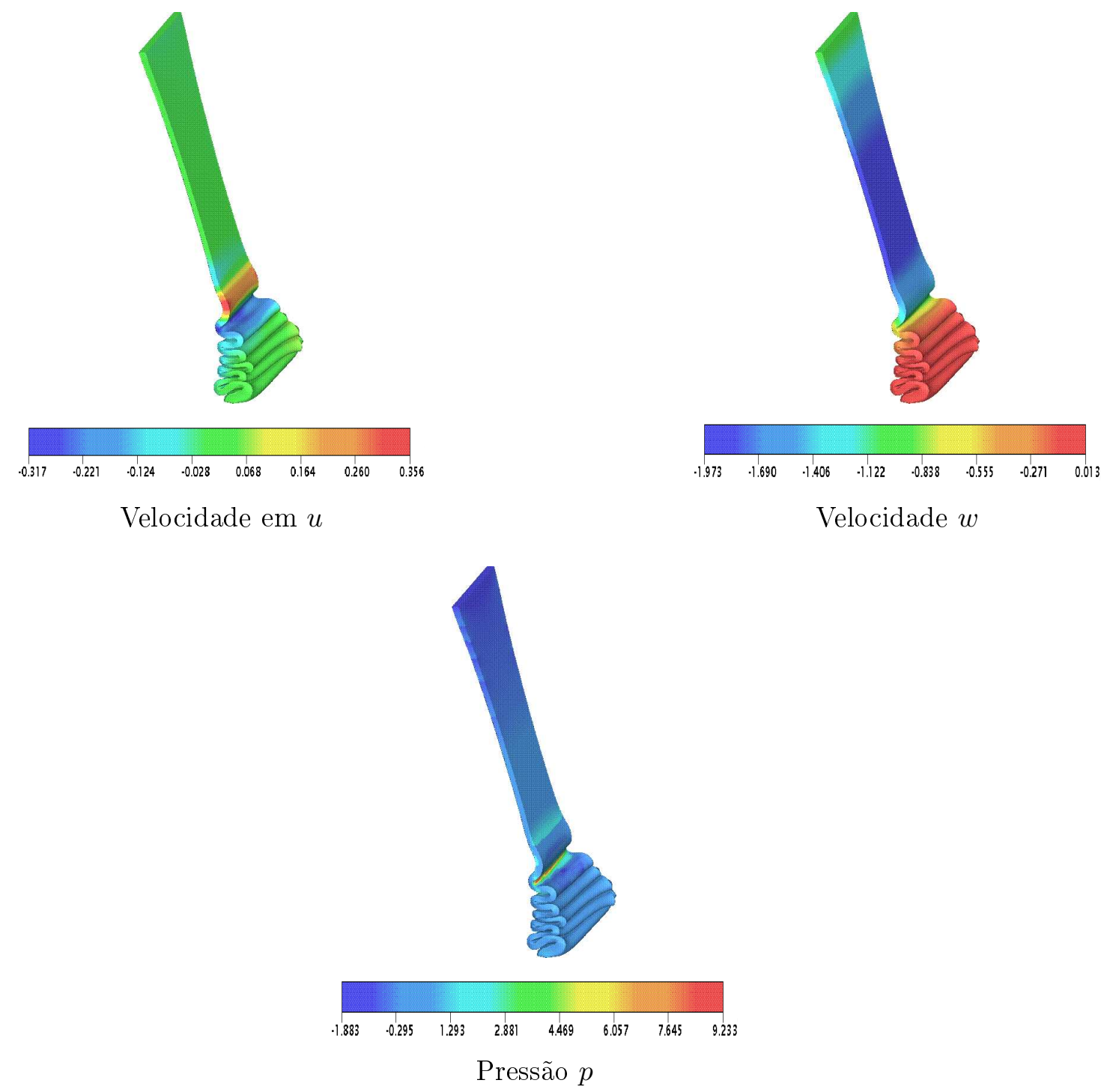

Figura 6.20: Contornos das velocidades $u, w$ e a pressão $p$ do jato planar oscilante. $t=1.2 s$. 


\subsubsection{Jatos livres $2 \mathrm{D}$ e $3 \mathrm{D}$ no regime turbulento}

O novo esquema TDPUS-C3 é avaliado agora na simulação de jatos livres sobre superfícies impermeáveis (ver Fig. 6.1 (b)) no regime turbulento. O problema físico considerado é o salto hidráulico circular em ambos os casos 2D e 3D. Para a simulação computacional desses problemas complexos os modelos $\kappa-\varepsilon$ padrão (Launder e Spalding, 1974) e $R N G \kappa-\varepsilon$ (Yakhot e Orszag, 1986), ambos associados com o esquema TDPUS-C3 para as não linearidades, foram implementados no ambiente de simulação Freeflow (2D e 3D). Junto ao contorno rígido, isto é na primeira camada de células computacionais, adotou-se as leis de parede descritas na seção 4.4.2.3. Para os outros contornos (superfície livre e saída de fluido) aplicaram-se as mesmas equações como no caso do problema em regime laminar, considerando o tensor $\sigma$ correspondente (ver Eq. 4.120). No caso 2D, os dados adotados nas simulações estão apresentados na Tab. 6.8 .

Tabela 6.8: Dados da simulação do jato livre 2D no regime turbulento.

- Malha I : $200 \times 50$ células computacionais;

- Malha II : $400 \times 100$ células computacionais;

- Malha III : $800 \times 200$ células computacionais;

- Raio do injetor: $a=0.005 m$;

- Domínio: $0.2 \mathrm{~m} \times 0.05 \mathrm{~m}$;

- Escala de comprimento: $L_{0}=2 a=0.01 \mathrm{~m}$;

- Escala de velocidade: $U_{0}=1 \mathrm{~ms}^{-1}$;

- Coeficiente de viscosidade cinemática: $\nu=0.000002 m^{2} s^{-1}$;

- Número de Froude: $F r \approx 3.19$;

- Número de Reynolds: $R e=50000$.

Da mesma forma como no caso do salto hidráulico em regime laminar, o problema no regime turbulento tem solução analítica dada por Watson (1964) para a altura $h$ de fluido acima do contorno rígido

$$
\begin{gathered}
h(x)= \begin{cases}\frac{81(7 A)^{1 / 4} k}{800} \cdot\left(\frac{\nu}{Q}\right)^{1 / 4}(x+l), & x \geq x_{0} ; \\
a+\left(1-\frac{A}{k}\right) \delta(x), & x<x_{0},\end{cases} \\
x_{0}=\frac{320(9 A-2)}{81 \times 7^{1 / 4} A^{5 / 4}} a R e^{1 / 4} ; \\
l=\frac{160(1-2 A)}{9 \times 7^{1 / 4} A^{5 / 4}} a R e^{1 / 4} ; \\
\delta(x)=\left(\frac{81}{320(9 A-2)}\right)^{4 / 5} 7^{1 / 5} k\left(\frac{a \nu}{Q}\right)^{1 / 5} x^{4 / 5} ; \\
A=0.239 ; \quad k=0.26 .
\end{gathered}
$$

A Fig. 6.21 mostra a comparação entre as soluções numéricas nas malhas I, II e III obtidas com o 
modelo $\kappa-\varepsilon$ padrão associado ao TDPUS-C3 e a solução analítica de Watson. Como pode ser visto, as soluções numéricas nas malhas estão em boa concordância com a solução analítica; em particular, a solução numérica na malha III está bastante próxima da solução de Watson no intervalo $[0.45,0.6]$. A Fig. 6.22 mostra a mesma comparação apresentada na Fig. 6.21, só que com o modelo $R N G \kappa-\varepsilon$ no lugar do $\kappa-\varepsilon$ padrão, onde pode-se notar que houve também uma boa concordância com a solução analítica de Watson. A Fig. 6.23 mostra a comparação entre as soluções obtidas com os modelos de turbulência $\kappa-\varepsilon$ padrão e o modelo $R N G \kappa-\varepsilon$ na malha I. Vê-se claramente por essa figura que a solução numérica obtida com o modelo $\kappa-\varepsilon$ padrão está mais próxima da solução analítica que à obtida com o modelo $R N G \kappa-\varepsilon$.

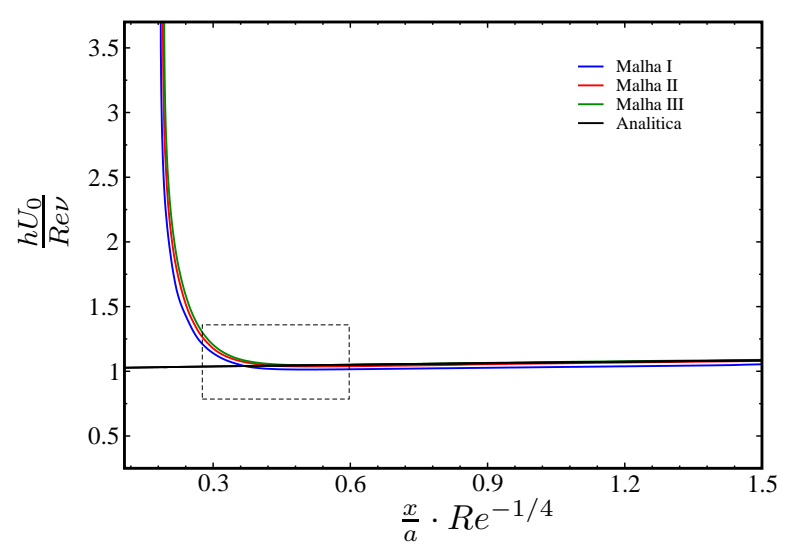

(a)

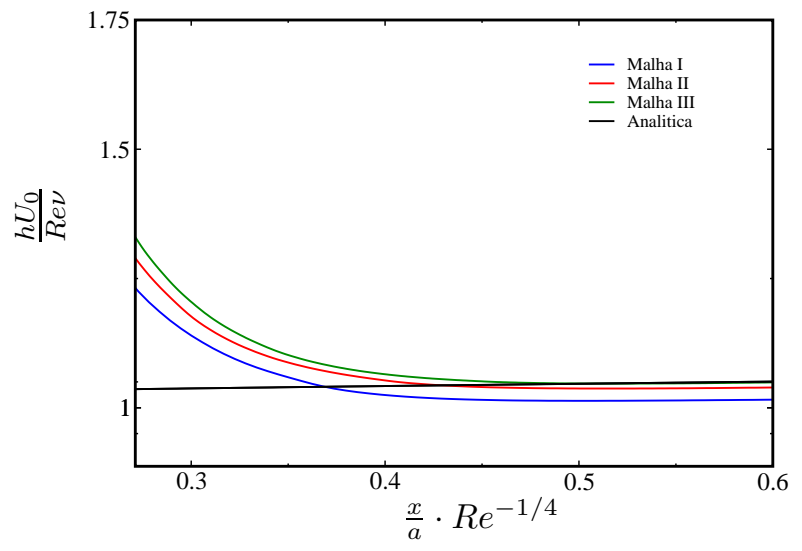

(b)

Figura 6.21: Comparação entre as soluções numéricas e analítica para o problema do jato livre 2D sobre uma superfície rígida no modelo $\kappa-\varepsilon$ padrão associado ao TDPUS-C3 (a) à esquerda; ampliação da região destacada na Fig. (a), à direita.

As Figs. 6.24-6.26 e 6.27-6.29 ilustram os contornos dos componentes da velocidade $u, v, w$ e o campo de pressão $p$, em diferentes tempos, obtidos com os modelos $\kappa-\varepsilon$ padrão e $R N G \kappa-\varepsilon$, respectivamente. Observa-se que o fluido espalha mais quando o modelo $R N G \kappa-\varepsilon$ é utilizado na simulação; isso é uma indicação de que a modelagem $R N G \kappa-\varepsilon$ - TDPUS-C3, aplicada a esse problema, introduz menos viscosidade numérica. 


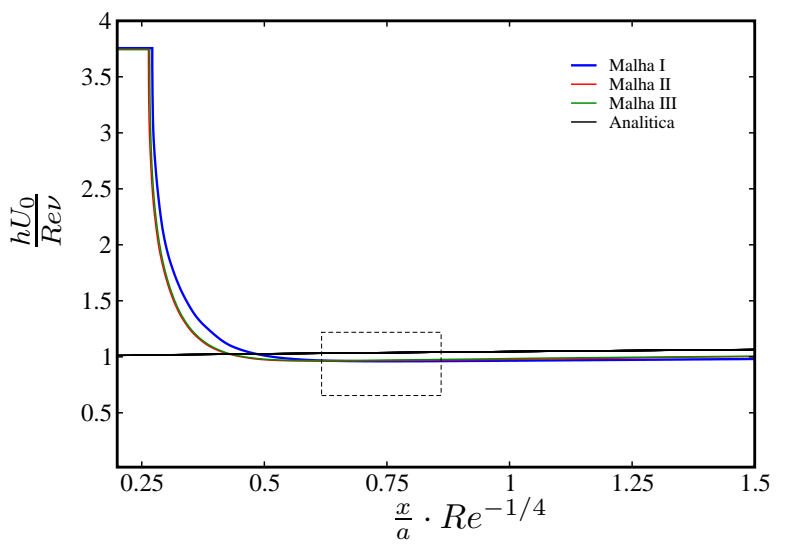

(a)

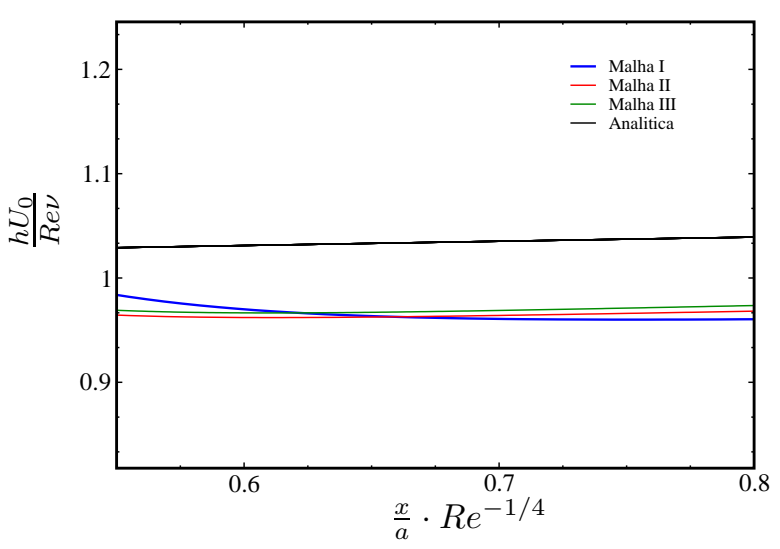

(b)

Figura 6.22: Comparação entre as soluções numéricas e analítica para o problema do jato livre 2D sobre uma superfície rígida impermeável no modelo $R N G \kappa-\varepsilon$ associado ao TDPUS-C3, à esquerda; ampliação da região destacada na Fig. (a), à direita.

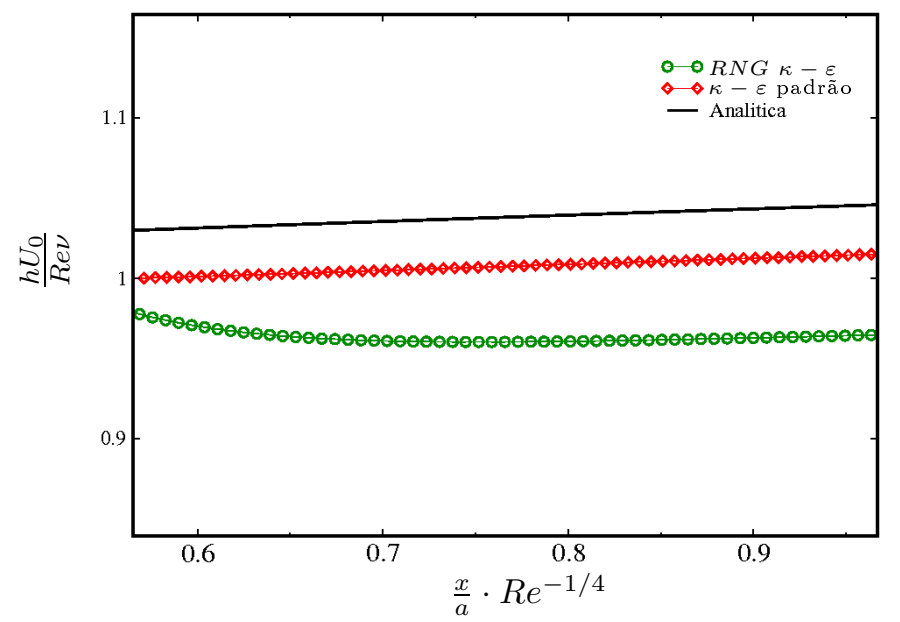

Figura 6.23: Comparação entre as soluções numéricas obtidas com TDPUS-C3 nos modelos $\kappa-\varepsilon$ padrão e $R N G \kappa-\varepsilon$ associados ao TDPUS-C3, e analítica do problema do jato livre 2D sobre uma superfície rígida impermeável. Malha I. 

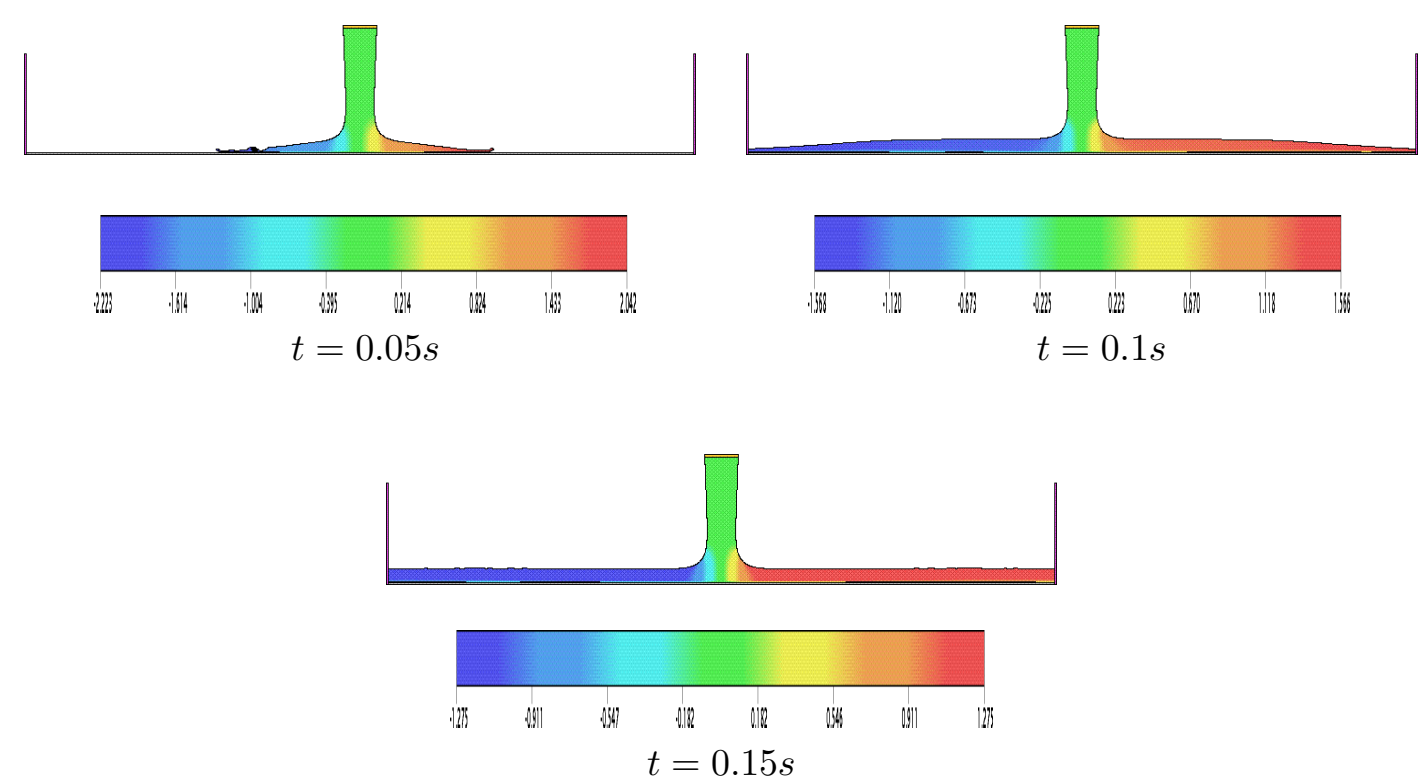

Figura 6.24: Contorno da velocidade $u$ para o problema do jato livre 2D sobre uma superfície rígida impermeável no modelo $\kappa-\varepsilon$ padrão associado ao TDPUS-C3. Malha III.
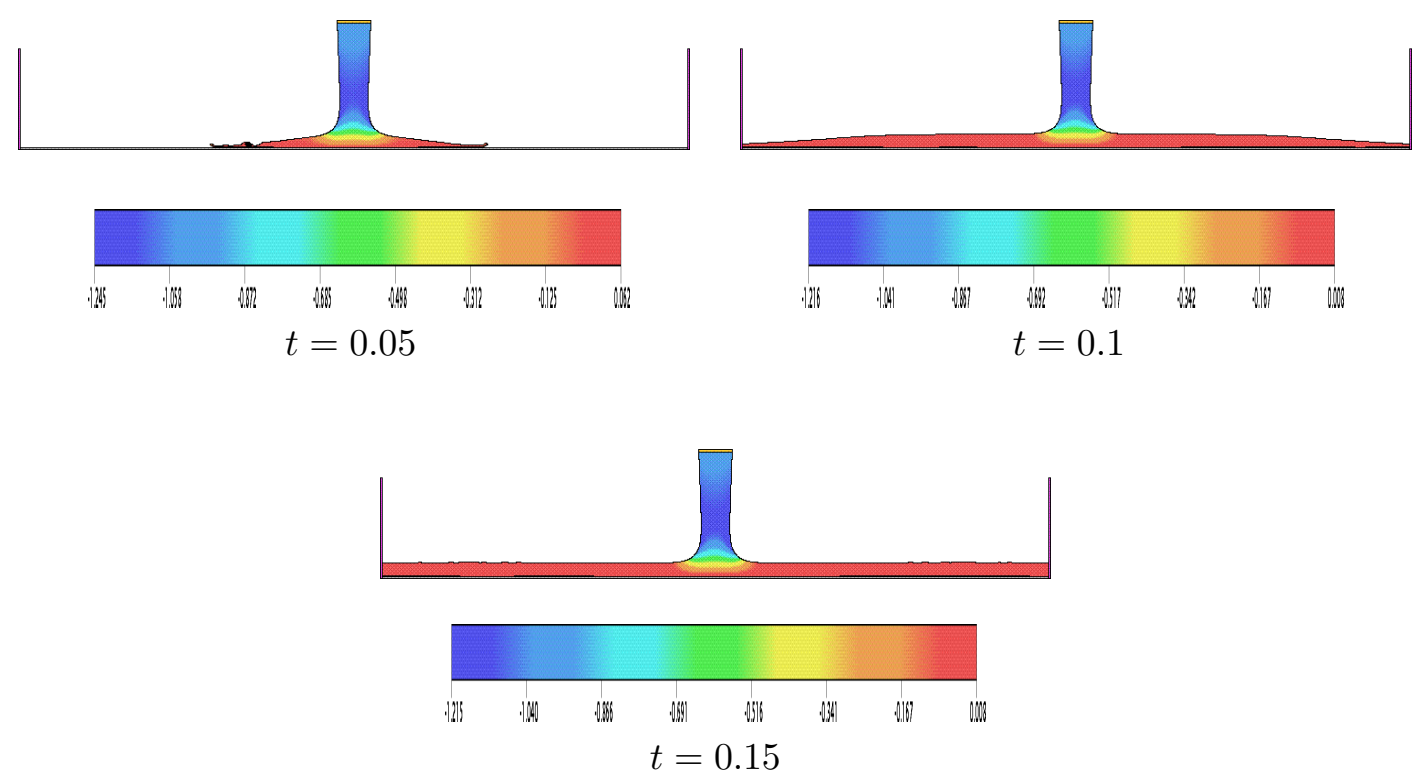

Figura 6.25: Contorno da velocidade $v$ para o problema do jato livre 2D sobre uma superfície rígida impermeável no modelo $\kappa-\varepsilon$ padrão associado ao TDPUS-C3. Malha III. 

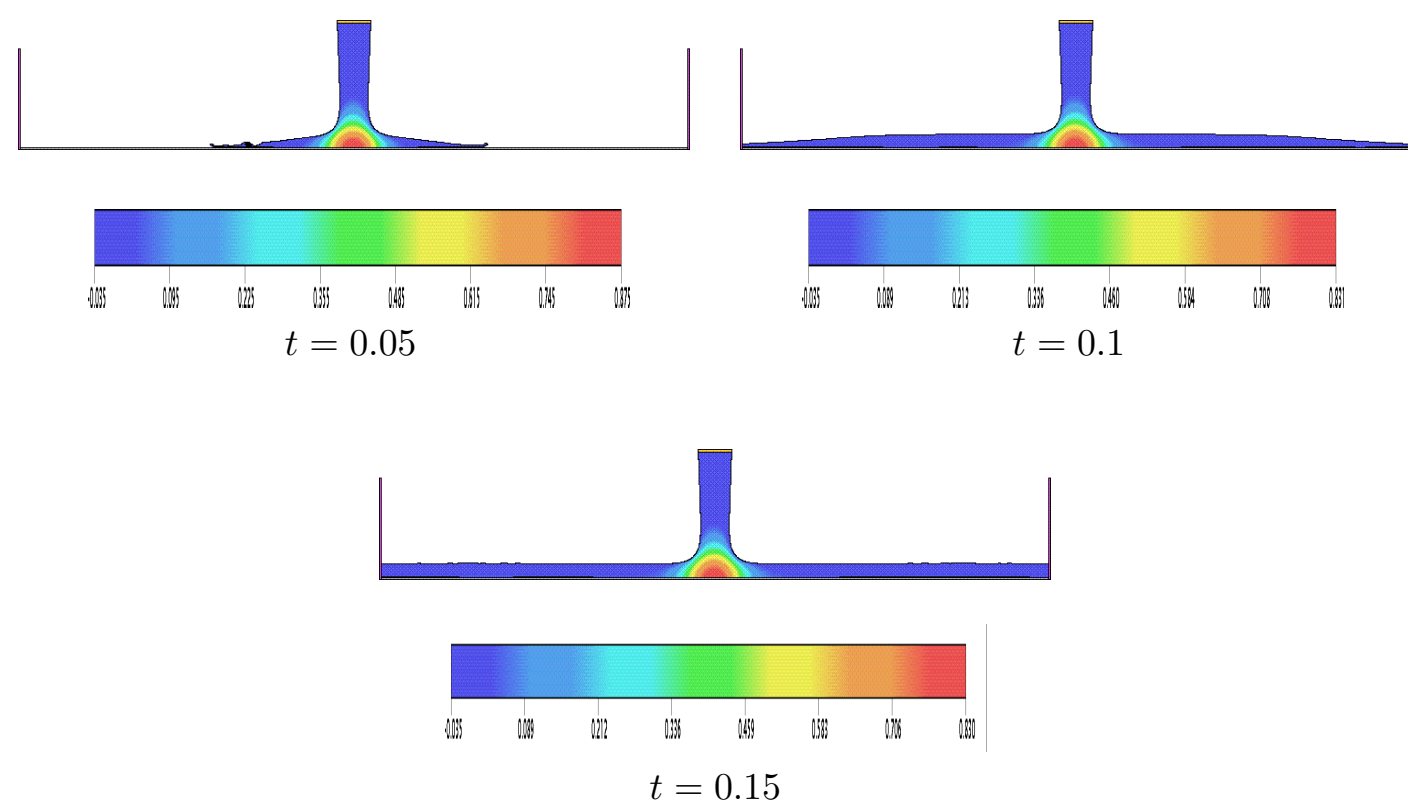

Figura 6.26: Contorno da pressão $p$ para o problema do jato livre 2D sobre uma superfície rígida impermeável no modelo $\kappa-\varepsilon$ padrão associado ao TDPUS-C3. Malha III.
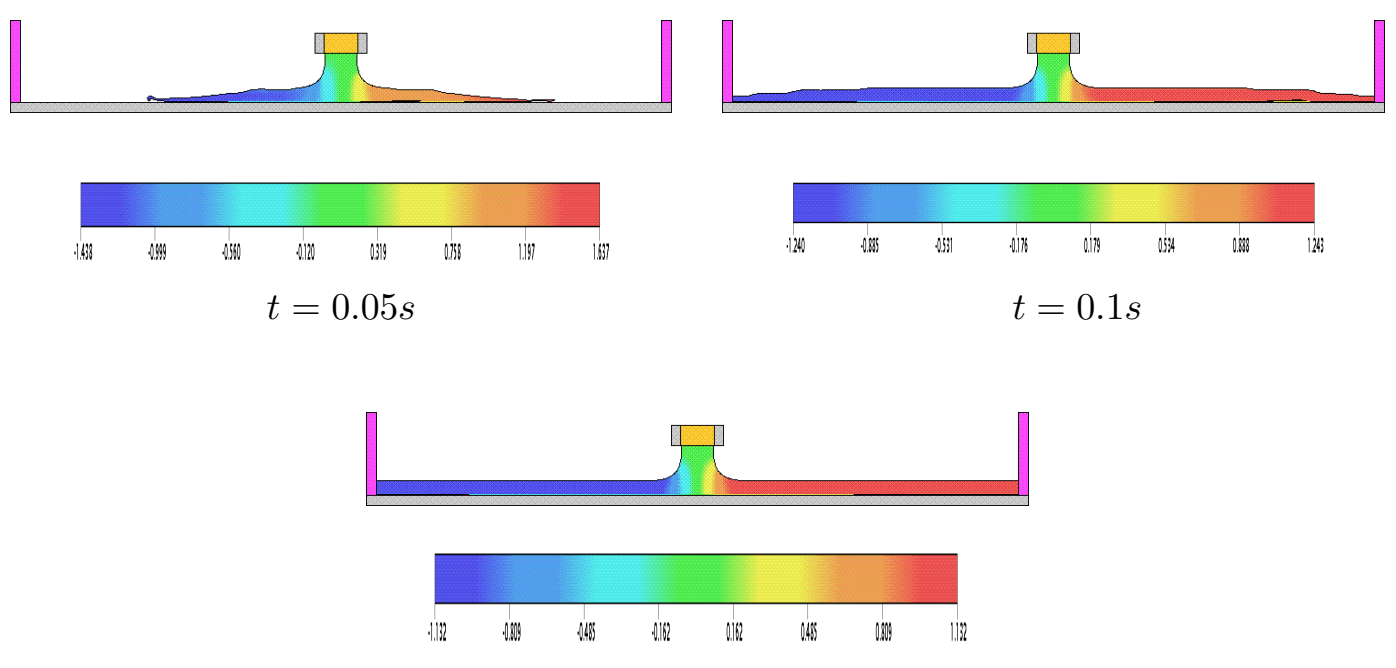

$t=0.15 s$

Figura 6.27: Contorno da velocidade $u$ para o problema do jato livre 2D sobre uma superfície rígida impermeável no modelo $R N G \kappa-\varepsilon$ associado ao TDPUS-C3. Malha III. 

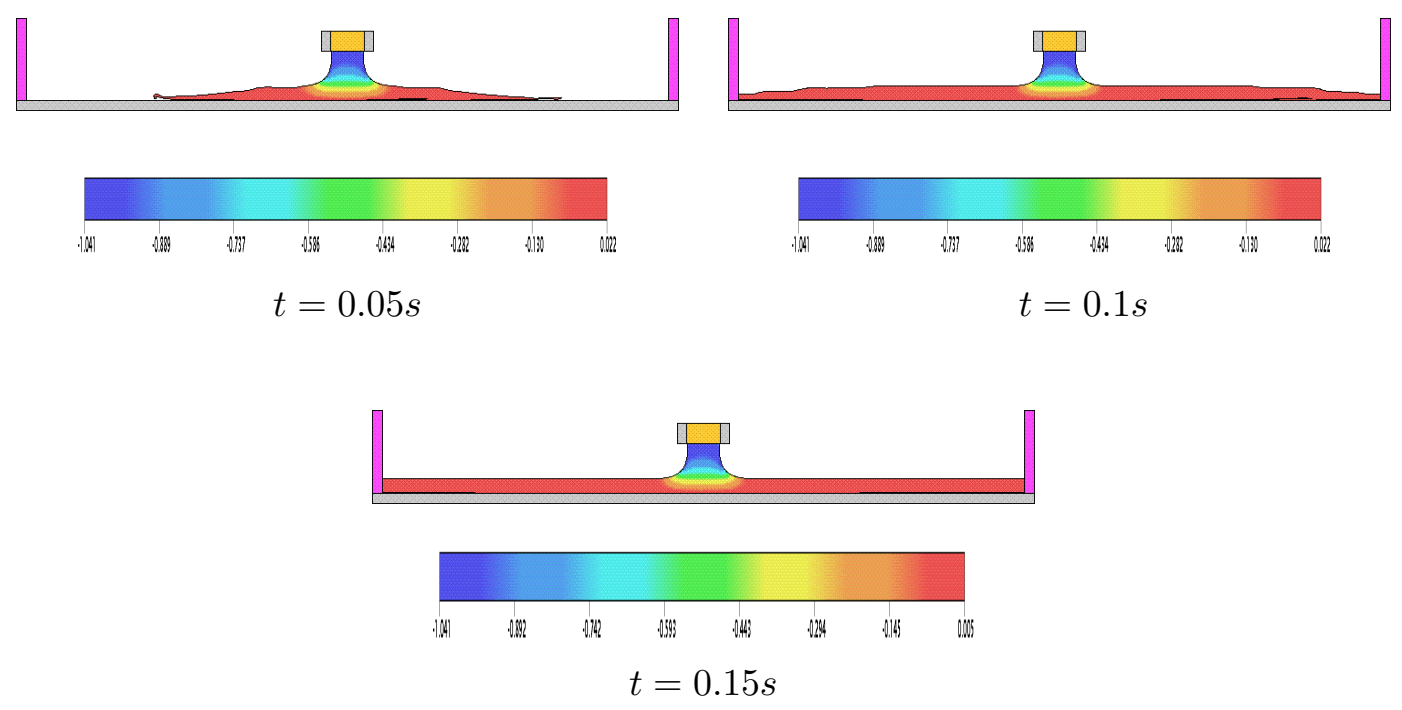

Figura 6.28: Contorno da velocidade $v$ para o problema do jato livre 2D sobre uma superfície rígida impermeável no modelo $R N G \kappa-\varepsilon$. Malha III.
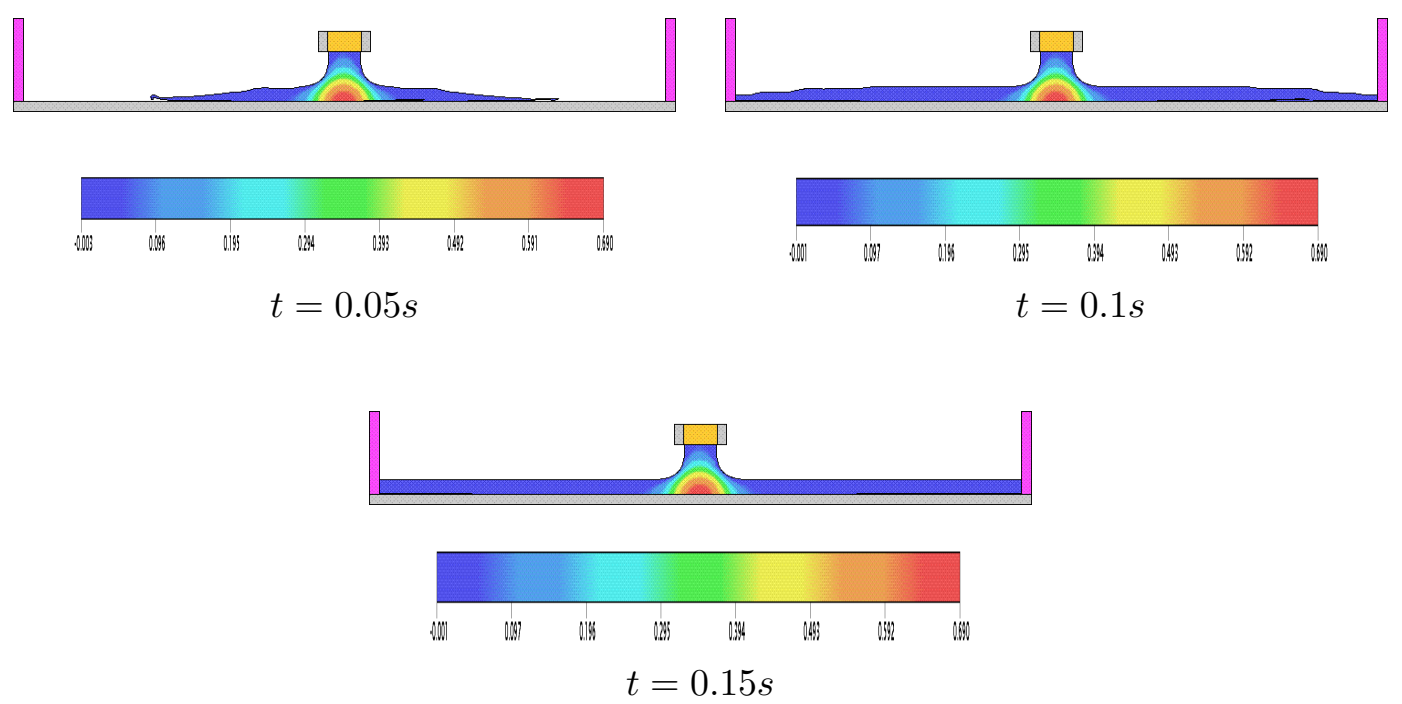

Figura 6.29: Contorno da pressão $p$ para o problema do jato livre 2D sobre uma superfície rígida impermeável no modelo $R N G \kappa-\varepsilon$ associado ao TDPUS-C3. Malha III. 
No caso da simulação de jatos livres 3D, os dados adotados estão apresentados na Tab. 6.9.

Tabela 6.9: Dados da simulação do jato livre 3D no regime turbulento.

- Malha I: $50 \times 50 \times 10$ células computacionais;

- Malha II: $100 \times 100 \times 20$ células computacionais;

- Domínio: $0.1 \mathrm{~m} \times 0.1 \mathrm{~m} \times 0.02 \mathrm{~m}$;

- Escala de comprimento: $L_{0}=0.01 \mathrm{~m}$;

- Escala de velocidade: $U_{0}=2.0 \mathrm{~ms}^{-1}$;

- Coeficiente de viscosidade cinemática: $\nu=0.000000625 m^{2} s^{-1}$;

- Constante gravitacional: $g=9.81 \mathrm{~ms}^{-2}$.

- Diâmetro do injetor: $D=0.01 \mathrm{~m}$.

- Altura do injetor a partir da superfície rigida: $H=0.015 \mathrm{~m}$.

- Número de Froude: $F r \approx 0.64$

- Número de Reynolds: $R e=\frac{\pi}{2} \frac{U_{0} L_{0}}{\nu} \approx 50.000$;

Utilizou-se neste caso 3D, para comparações, a seguinte solução analítica de Watson (1964) derivada para escoamentos turbulentos em geometrias com simetria radial:

$$
\begin{gathered}
h(x)= \begin{cases}\frac{9 k}{200}(14 \pi A)^{1 / 4}\left(\frac{x^{9 / 4}+l^{9 / 4}}{x}\right), & x \geq x_{0} ; \\
\frac{1}{2 x}+\left(\frac{k-A}{k}\right) \delta, & x \leq x_{0},\end{cases} \\
\delta=\left[80\left(A-\frac{2}{9}\right)\right]^{-4 / 5} k(7 \pi)^{1 / 5} x^{4 / 5},
\end{gathered}
$$

onde $k=0.260$ e $A=0.239$. O valor de $x_{0}$ é dado por

$$
x_{0}=(7 \pi)^{-1 / 9}(2 A)^{-5 / 9}\left[80\left(A-\frac{2}{9}\right)\right]^{4 / 9}
$$

onde $l=20(1-2 A)(14 \pi)^{-1 / 4} A^{-5 / 4}$.

As Figs. 6.30 e 6.31 mostram as comparações entre as soluções numéricas (extraídas no plano yz na posição $x=0.056$ ), nas malhas I e II, obtidas com TDPUS-C3 em associação com os modelos de turbulência $\kappa-\varepsilon$ padrão e $R N G \kappa-\varepsilon$ e as soluções (viscosa e não viscosa) de Watson. Como pode-se ver por essas figuras, ambos os modelos de turbulência forneceram resultados semelhantes, com o modelo $\kappa-\varepsilon$ padrão um pouco melhor; isso já tinha sido observado no caso 2D (ver Fig. 6.23). Em particular, vê-se pela Fig. 6.31 (b) que ao se refinar a malha (de I para II) houve uma tendência em se obter convergência da solução numérica. Vale observar aqui que os resultados numéricos em uma malha mais fina, tal como uma Malha III de $200 \times 200 \times 40$ células computacionais, foram proibitivos de se obter, pois para estabilizar os cálculos o tamanho do passo temporal $\delta t$ assumiu valores menores ou iguais a $10^{-7}$.

Como ilustração, a Fig. 6.32 apresenta a evolução temporal da superfície livre do fluido usando o modelo de turbulência $R N G \kappa-\varepsilon$ associado com TDPUS-C3 na Malha II. As Figs. 6.33-6.35 ilustram, na Malha II, os contornos dos componentes da velocidade $u, w$ e campo de pressão $p$ obtidos 
com os modelos $\kappa-\varepsilon$ padrão e $R N G \kappa-\varepsilon$ associados com TDPUS-C3.
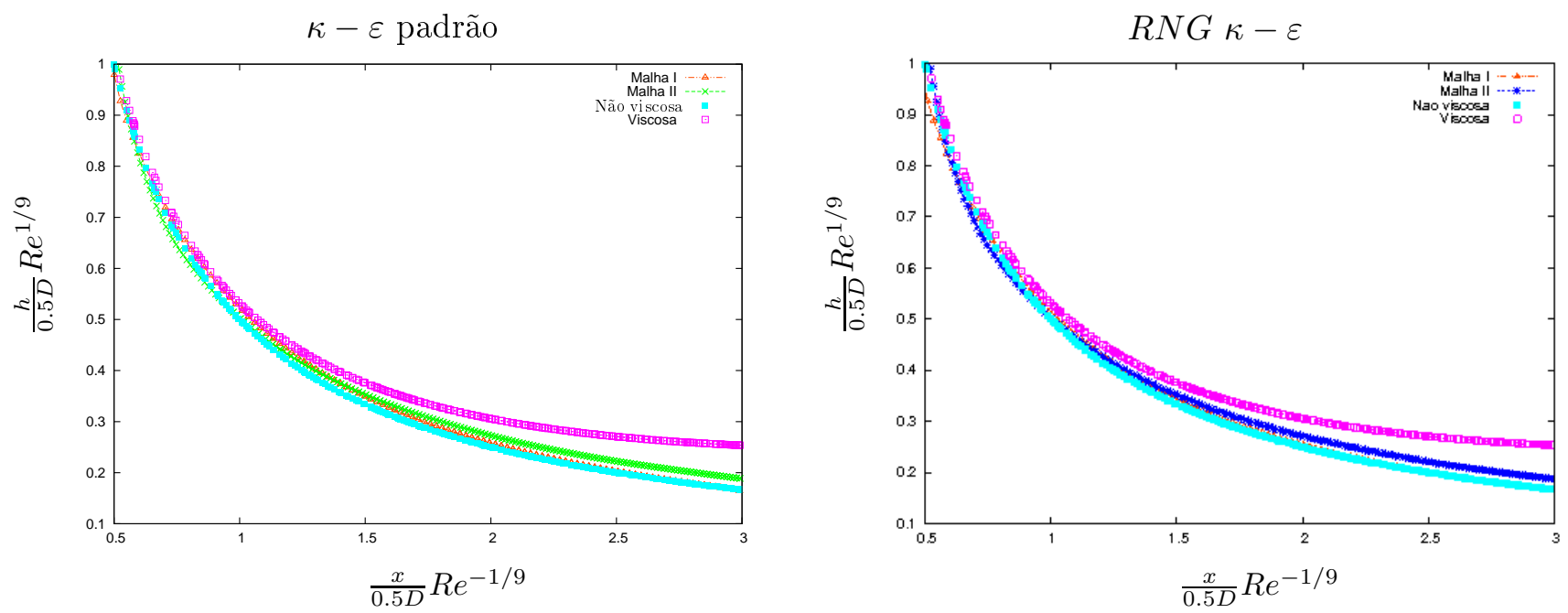

Figura 6.30: Soluções numéricas obtidas com TDPUS-C3 (extraídas no plano $y z$ na posição $x=0.056$ ) para o problema do jato livre 3D com os modelos de turbulência $\kappa-\varepsilon$ padrão e $R N G \kappa-\varepsilon$ associados com TDPUS-C3, comparadas com as soluções viscosa e não viscosa de Watson (1964).

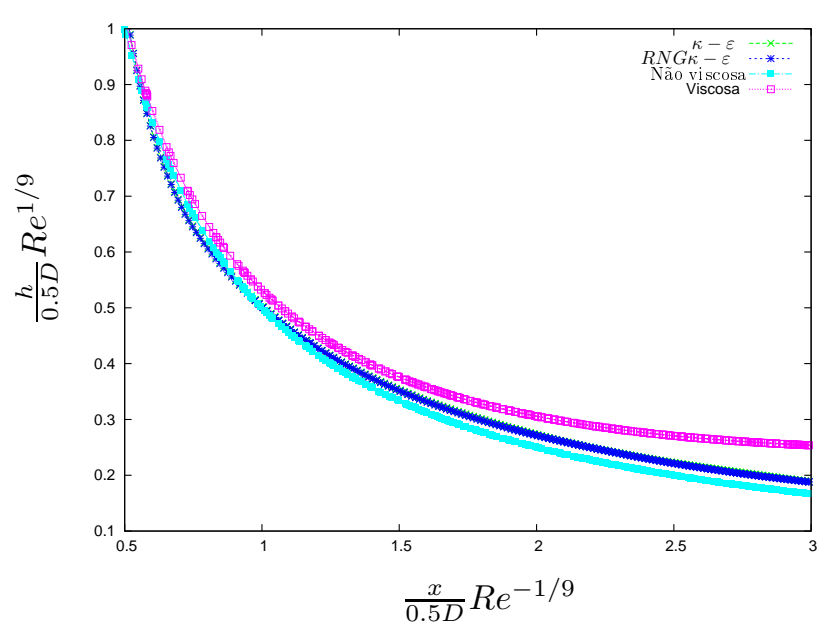

(a)

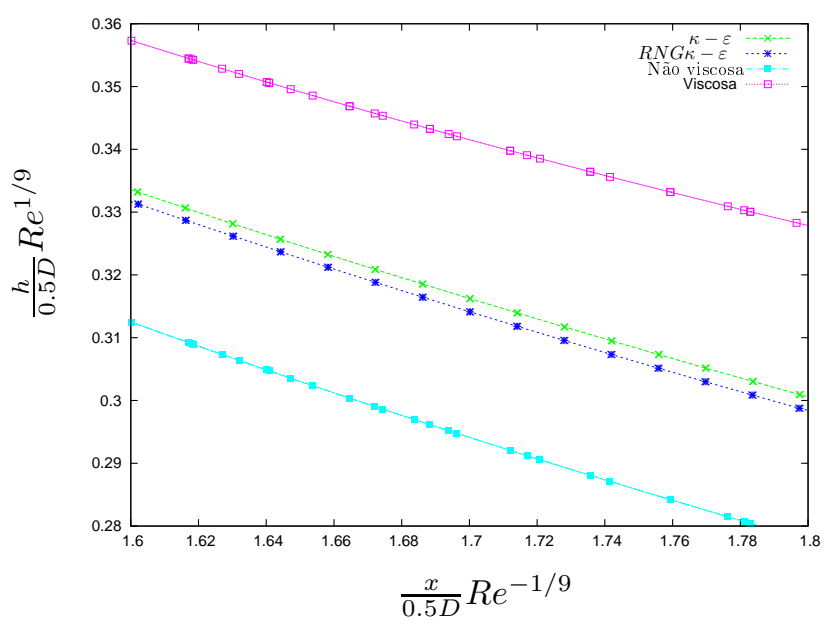

(b)

Figura 6.31: Comparação entre as soluções numéricas (extraídas no plano yz na posição $x=0.056$ ) obtidas com TDPUS-C3 e as soluções analíticas não viscosa e viscosa de Watson (1964) para o problema do jato livre 3D com os modelos $\kappa-\varepsilon$ padrão e $R N G \kappa-\varepsilon$ associados ao TDPUS-C3, à esquerda; ampliação da região destacada na Fig. (a), à direita. Malha II. 


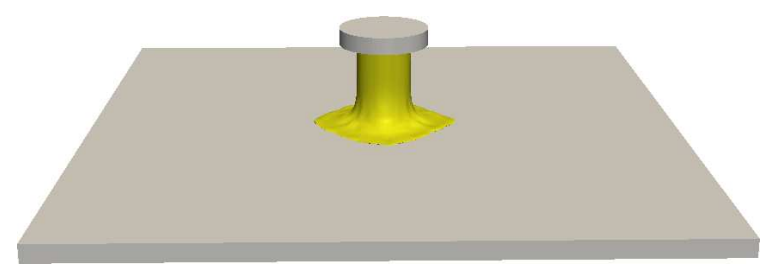

$t=0.01 s$

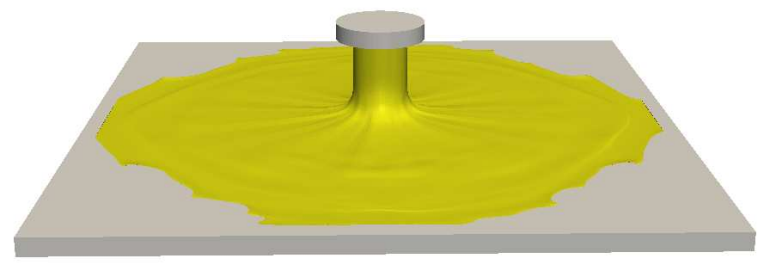

$t=0.03 \mathrm{~s}$

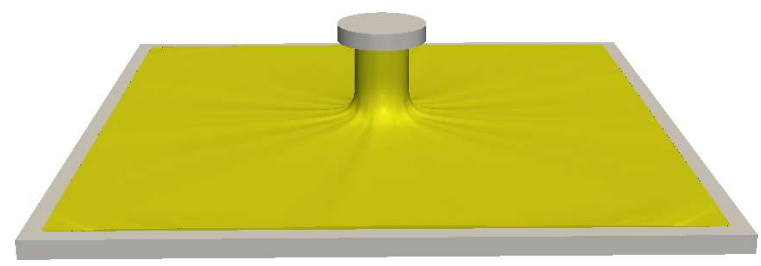

$t=0.05 s$

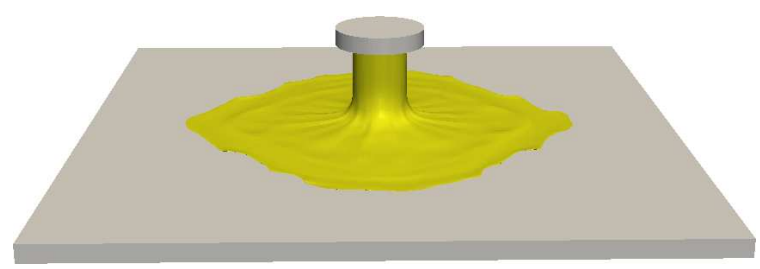

$t=0.02 s$

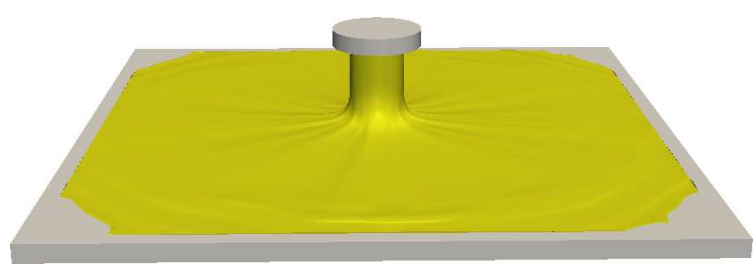

$t=0.04 \mathrm{~s}$

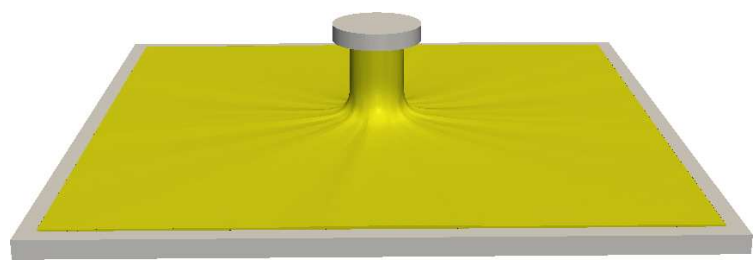

$t=0.06 s$

Figura 6.32: Evolução temporal do problema do jato livre 3D sobre uma superfície rígida impermeável usando a associação $R N G \kappa-\varepsilon$-TDPUS-C3. Malha II. 

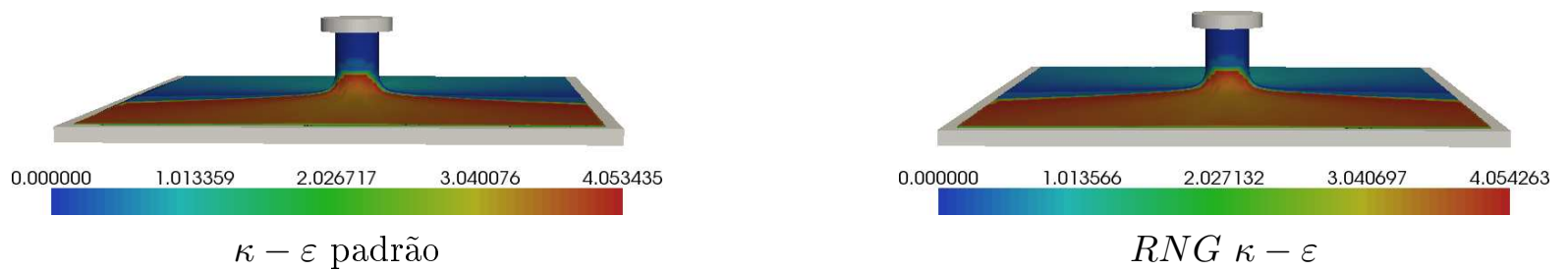

Figura 6.33: Contorno da velocidade $u$ para o problema do jato livre 3D sobre uma superfície rígida impermeável usando as associações $\kappa-\varepsilon$ padrão-TDPUS-C3 e $R N G \kappa-\varepsilon$-TDPUS-C3. Malha II.
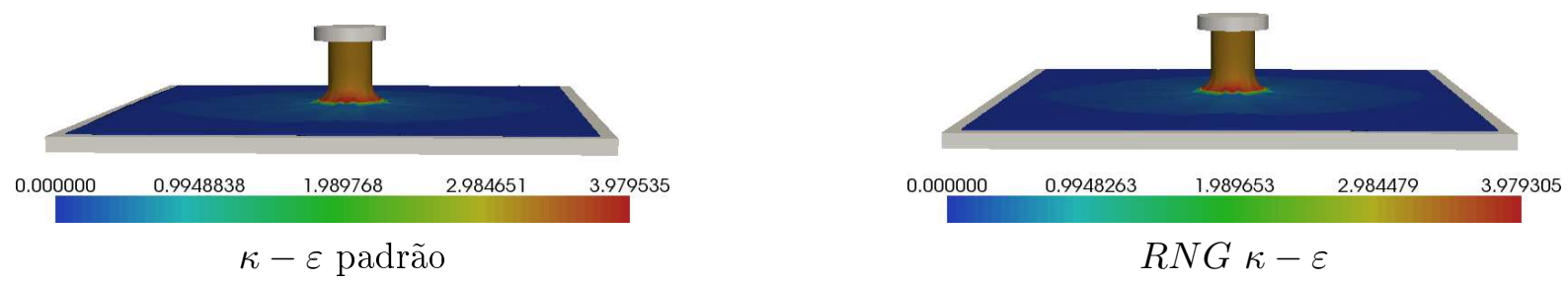

Figura 6.34: Contorno da velocidade $w$ para o problema do jato livre 3D sobre uma superfície rígida impermeável usando as associações $\kappa-\varepsilon$ padrão-TDPUS-C3 e $R N G \kappa-\varepsilon$-TDPUS-C3. Malha II.
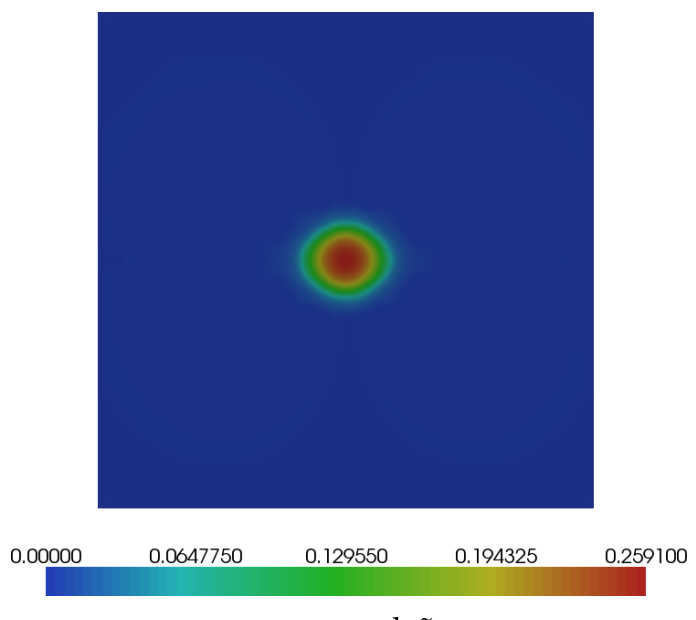

$\kappa-\varepsilon$ padrão
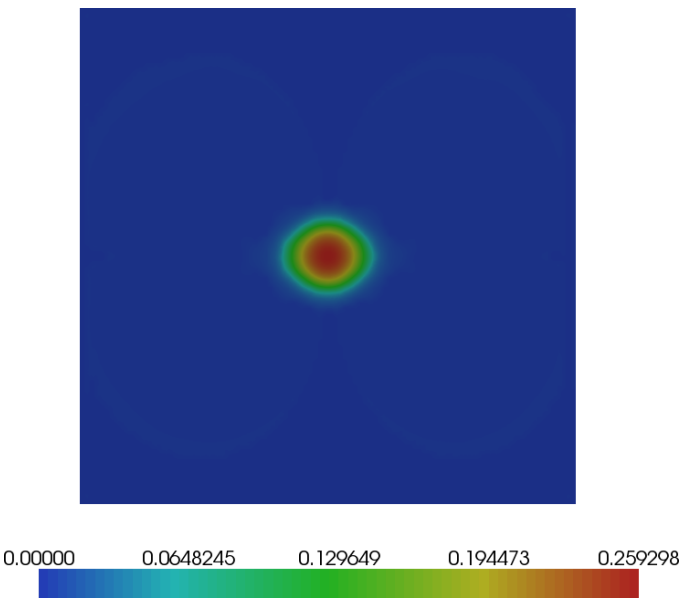

$$
R N G \kappa-\varepsilon
$$

Figura 6.35: Contorno da pressão $p$ para o problema do jato livre 3D sobre uma superfície rígida impermeável usando as associações $\kappa-\varepsilon$ padrão -TDPUS-C3 e $R N G \kappa-\varepsilon$-TDPUS-C3. Malha II. 


\subsubsection{Colapso de fluido e interação da superfície livre com um obstáculo}

Apresenta-se aqui o resultado computacional para o problema do colapso de um bloco de fluido e sua interação com um obstáculo 3D fixo. Para a simulação, foram considerados inicialmente dois blocos de fluidos (com as mesmas viscosidades) em repouso, sob a influência do campo gravitacional, como apresentado na Figs. 6.36 e 6.37 em $t=0$. O problema foi modelado considerando um tanque de dimensões $1.6 m \times 0.6 m \times 0.6 m$ e os dois blocos de fluido de dimensões $0.3 m \times 0.6 m \times 0.3 m$ e $1.3 m \times 0.6 m \times 0.03 m$ (ver Fig. 6.36); o obstáculo tem dimensões $0.12 m \times 0.12 m \times 0.6 m$ é está posicionado a uma distância de $0.5 \mathrm{~m}$ do bloco à esquerda e a $0.24 \mathrm{~m}$ das paredes laterais. Junto ao contorno rígido, foram adotadas as leis de parede descritas na seção 4.4.2.3, e na superfície livre do fluido aplicaram-se as mesmas equações dadas na Eq. 4.120, com o tensor $\sigma$ apropriado. A simulação foi realizada usando o código Freeflow 3D equipado com o esquema TDPUS-C3 e a modelagem $R N G \kappa-\varepsilon$.

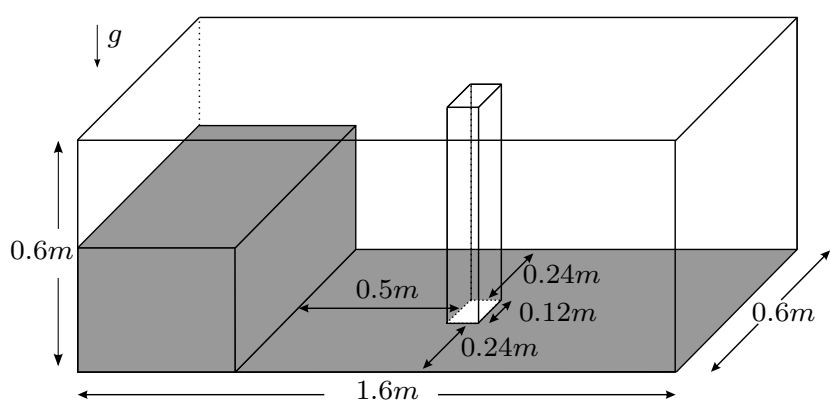

Figura 6.36: Ilustração do problema de colapso de fluido e interação da superfície livre com um obstáculo.

Os dados adotados na simulação estão apresentados na Tab. 6.10.

Tabela 6.10: Dados da simulação do colapso de fluido e interação da superfície livre com um obstáculo.

- Malha : $160 \times 60 \times 60$ células computacionais;

- Domínio: $1.6 m \times 0.6 m \times 0.6 m$;

- Escala de comprimento: $L_{0}=0.3 m$;

- Escala de velocidade: $U_{0}=\sqrt{g L_{0}}=1.715517 \mathrm{~ms}^{-1}$;

- Constante gravitacional: $g=9.81 \mathrm{~ms}^{-2}$.

- Coeficiente de viscosidade cinemática: $\nu=0.000001 m^{2} s^{-1}$;

- Número de Froude: $F r \approx 1.0$;

- Número de Reynolds: $R e \approx 514655.223$;

As Figs. 6.37 e 6.38 apresentam a evolução do escoamento (incluindo a onda antes da interação), mostrando a superfície livre móvel do fluido em duas posições tridimensionais diferentes: Fig. 6.37 mostrando vista lateral e Fig. 6.38 mostrando vista de cima. Esses dados, além de aparecerem visualmente realísticos, estão (qualitativamente) de acordo com os resultados apresentados por Raad e Bidoae (2005) via técnica Eulerian-Lagrangian marker and micro cell (ELMMC), e por Kurokawa (2009) via modelagem $\kappa-\varepsilon$ padrão. Em particular, nota-se na simulação apresentada na Fig. 6.37 a presença de estruturas turbilhonares (grandes vórtices) a partir do tempo $t=0.53 \mathrm{~s}$, os quais não são 
visíveis nas simulações de Raad e Bidoae (2005) e Kurokawa (2009). Em suma, as comparações entre os resultados apresentados aqui e àqueles por Raad e Bidoae (2005) (e também por Kurokawa (2009)) fornecem confiança na validade da combinação $R N G \kappa-\varepsilon$-TDPUS-C3.
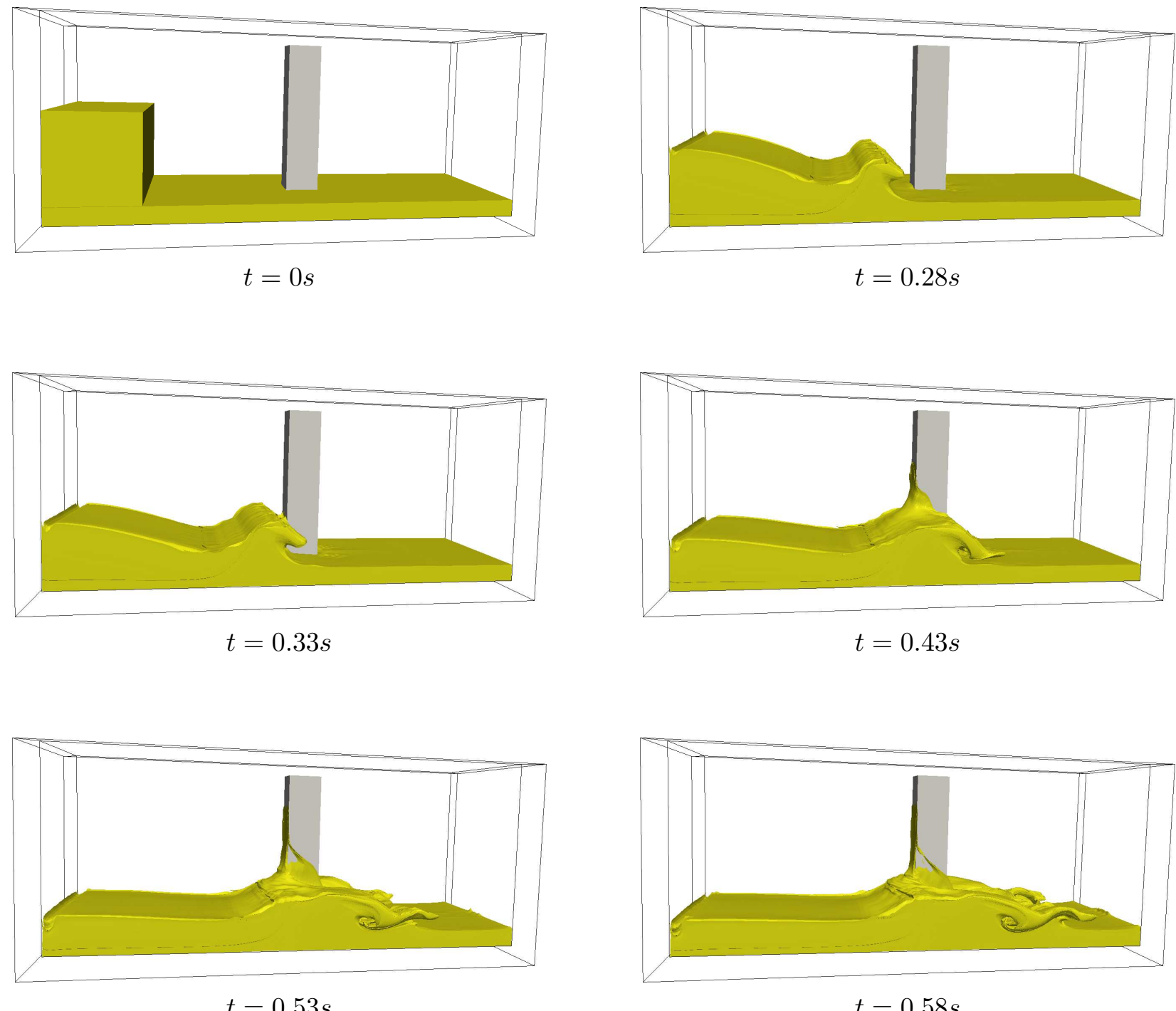

$t=0.53 s$

$$
t=0.58 s
$$
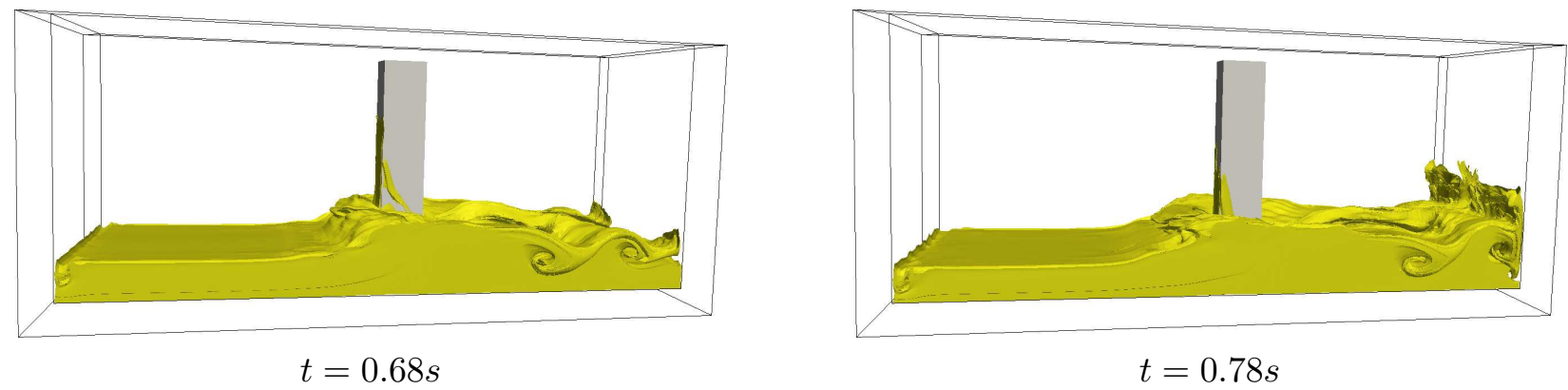

Figura 6.37: Evolução temporal das superfícies livres usando a combinação $R N G \kappa-\varepsilon$-TDPUS-C3 do problema de colapso de fluido e interação da superfície livre com um obstáculo. Vista lateral. 


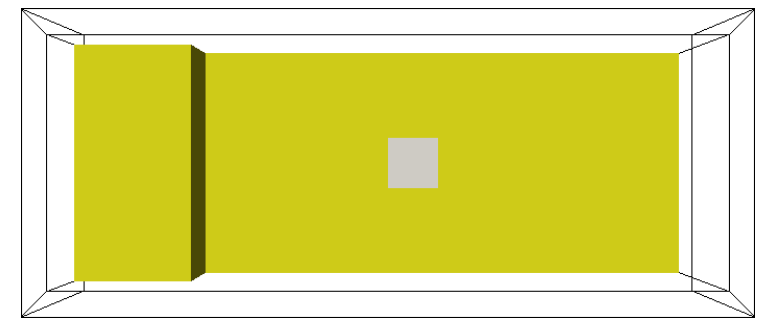

$t=0 s$

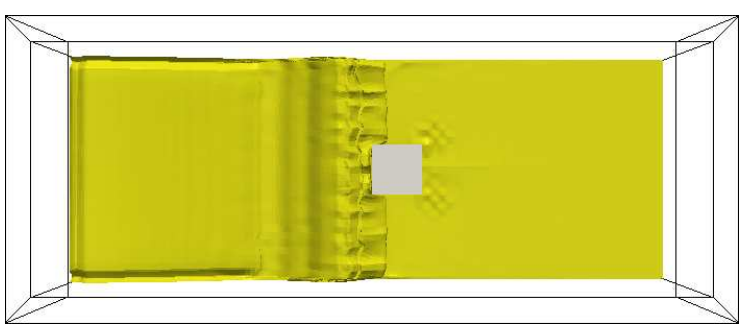

$t=0.33 \mathrm{~s}$

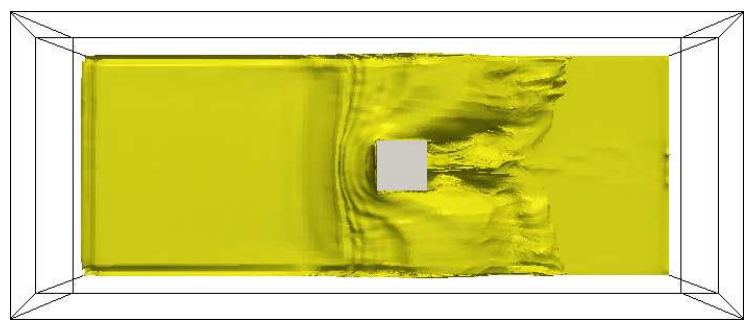

$t=0.53 s$

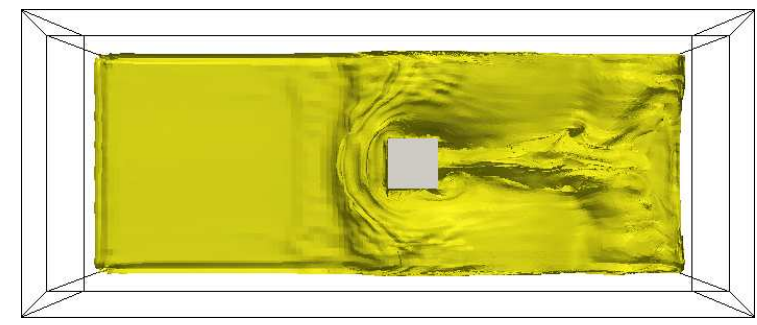

$t=0.68 s$

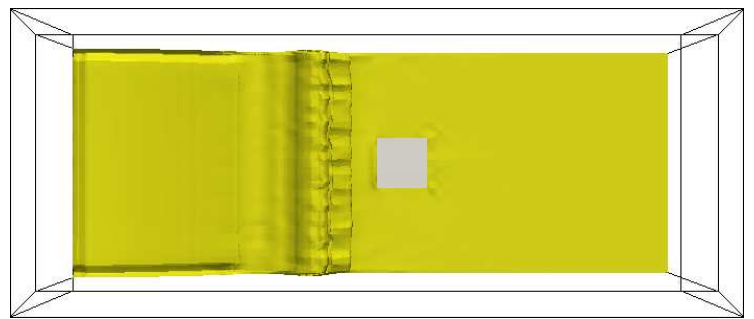

$t=0.28 s$

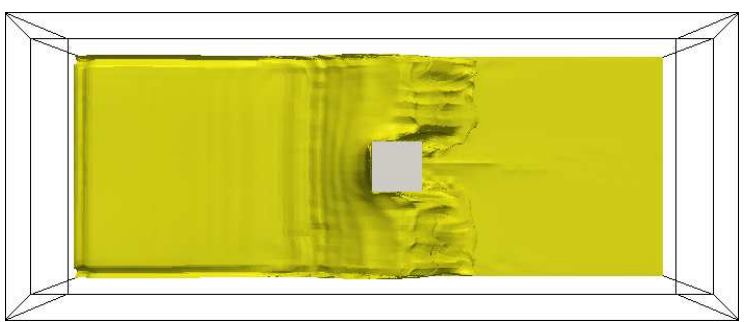

$t=0.43 s$

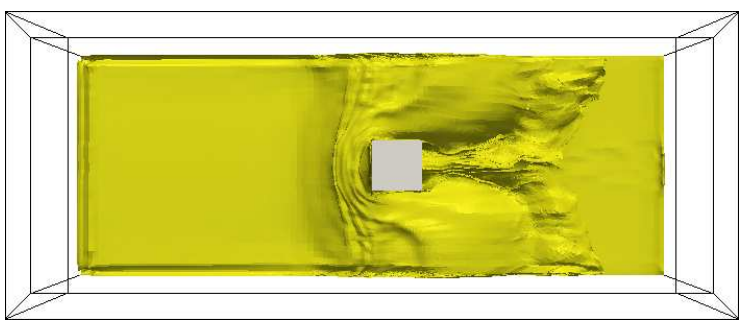

$t=0.58 s$

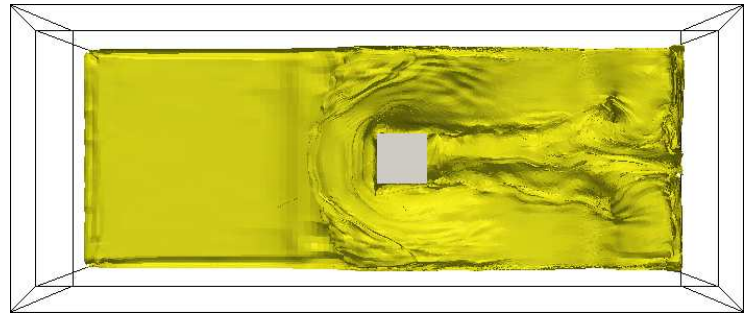

$t=0.78 s$

Figura 6.38: Evolução temporal das superfícies livres usando a combinação $R N G \kappa-\varepsilon$-TDPUS-C3 do problema de colapso de fluido e interação da superfície livre com um obstáculo. Vista de cima. 


\subsection{Resultados para fluidos não newtonianos 3D}

O problema do jato oscilante, o qual sob certas condições apresenta o fenômeno físico de dobras, já foi simulado usando o esquema TDPUS-C3 (ver seção 6.1.3) para o caso de fluidos newtonianos. Nesta seção apresentam-se resultados para o mesmo problema no caso de fluidos não newtonianos, em que foi selecionada a metodologia SXPP (Single eXtended Pom-Pom) de Verbeeten (2001) para modelar os efeitos viscoelásticos com características de elongamento e cisalhamento. Este modelo foi selecionado aqui por ser uma melhoria no modelo original Pom-Pom de McLeish e Larson (1998) e por ser uma técnica de modelagem com características de equações hiperbólicas. O objetivo em resolver numericamente este problema a baixo número de Reynolds é mostrar que é possível simular o fenômeno de dobras em fluidos viscoelásticos por uma escolha adequada dos parâmetros $\varrho$ (anisotropia do material), $\vartheta$ (razão entre viscosidades), $\gamma$ (razão entre constantes temporais) e $Q$ (número de ramificações). Os resultados numéricos foram obtidos usando uma versão implícita (o método Crank-Nicholson) do código Freeflow 3D adaptado com o esquema TDPUS-C3 e a número de Weissemberg $W e=0.1$. Esses dados foram comparados (qualitativamente) com os resultados obtidos com o uso do esquema CUBISTA de Alves et al. (2003) (ver Figueiredo (2011)).

A geometria considerada neste experimento numérico é ilustrada na Fig. 6.39, e os dados necessários para a simulação são como segue:

- Dimensões da caixa : $L \times L \times \frac{L}{2}$;

- Diâmetro do injetor: $d=0.16 L$;

- Distancia do fundo da caixa: $H=L$;

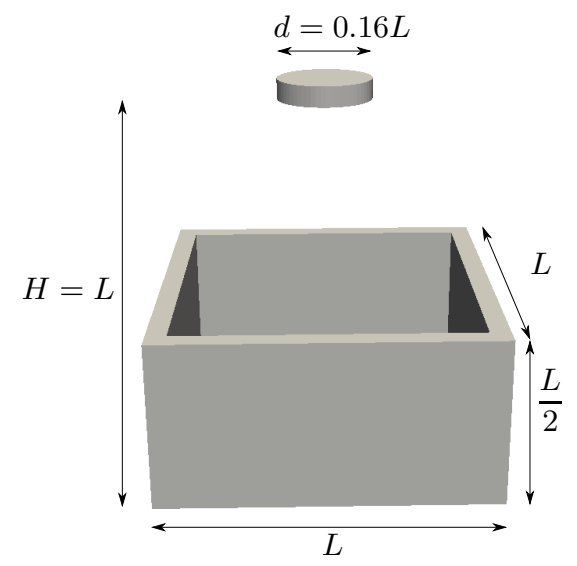

Figura 6.39: Geometria para o modelo SXPP.

onde $L=1 \mathrm{~m}$. As escalas de velocidade e de comprimento foram definidas como $U_{0}=1 \mathrm{~ms}^{-1} \mathrm{e}$ $L_{0}=d=0.16 m$, respectivamente. As condições de contorno adotadas foram de não escorregamento. A Tab. 6.11 apresenta os parâmetros adimensionais envolvidos no experimento e a ocorrência (ou não) do fenômeno de dobras.

As Figs. 6.40-6.43 apresentam as várias simulações realizadas, em vários instantes de tempo e a diferentes parâmetros, mostrando o escoamento do fluido viscoelástico SXPP. Observa-se que o fenômeno de dobras é um pouco mais evidente quando o número de Reynolds assumiu os valores 
Tabela 6.11: Parâmetros dos experimentos para o modelo SXPP.

\begin{tabular}{cccccc}
\hline \hline$R e$ & $\varrho$ & $\vartheta$ & $\gamma$ & $Q$ & Ocorrência de dobras (Sim/Não) \\
\hline 0.32 & 0.5 & 0.9 & 0.1 & 1 & Sim \\
0.64 & 0.5 & 0.9 & 0.1 & 1 & Sim \\
0.96 & 0.5 & 0.9 & 0.1 & 1 & Não \\
0.96 & 0.01 & 0.3 & 0.8 & 8 & Sim \\
\hline \hline
\end{tabular}

$R e=0.64$ e 0.32 (ver Figs.6.42 e 6.43). Para o caso em que os adimensionais $R e, \varrho, \vartheta$, $\gamma$ e $Q$ assumem, respectivamente, os valores $R e=0.96, \varrho=0.01, \vartheta=0.3, \gamma=0.8$ e $Q=8$, o fenômeno de dobras é menos evidente (ver Fig.6.41). O fenômeno das dobras não apareceu quando os adimensionais assumiram os valores $R e=0.96, \varrho=0.5, \vartheta=0.9, \gamma=0.1$ e $Q=1$ (ver Fig.6.40). A título de uma comparação qualitativa, a Fig. 6.44 extraída de Figueiredo (2011) apresenta, nos tempos $t=2 s$, $5 s$ e $9 s$, a simulação computacional desse mesmo problema com o uso do esquema CUBISTA, onde pode ser visto que os resultados obtidos com o esquema TDPUS-C3 mostrados na Fig. 6.43 foram, praticamente, idênticos. Portanto, pode-se dizer que o esquema TDPUS-C3 é útil também para simular escoamentos de fluidos viscoelásticos em 3D.
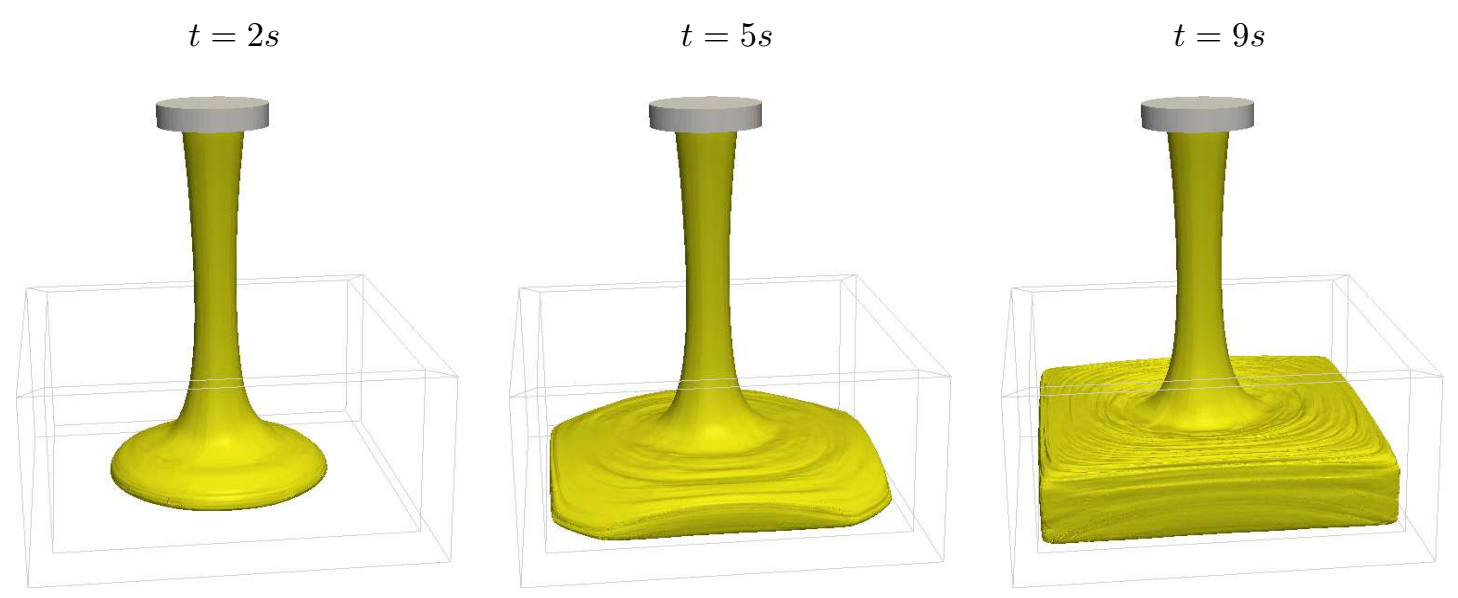

Figura 6.40: Modelo SXPP. Resultados obtidos com TDPUS-C3. Re $=0.96, W e=0.1, \varrho=0.5$, $\vartheta=0.9, \gamma=0.1, Q=1$. 


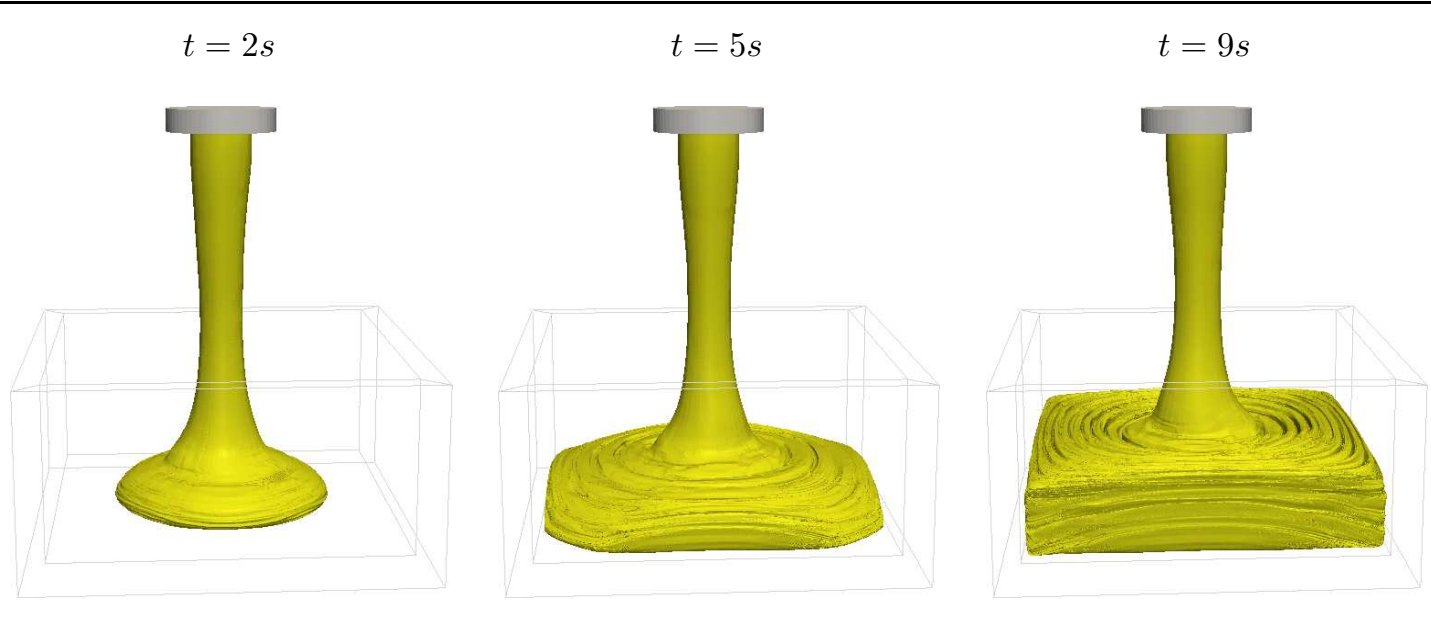

Figura 6.41: Modelo SXPP. Resultados obtidos com TDPUS-C3. Re $=0.96, W e=0.1, \varrho=0.01$, $\vartheta=0.3, \gamma=0.8$.
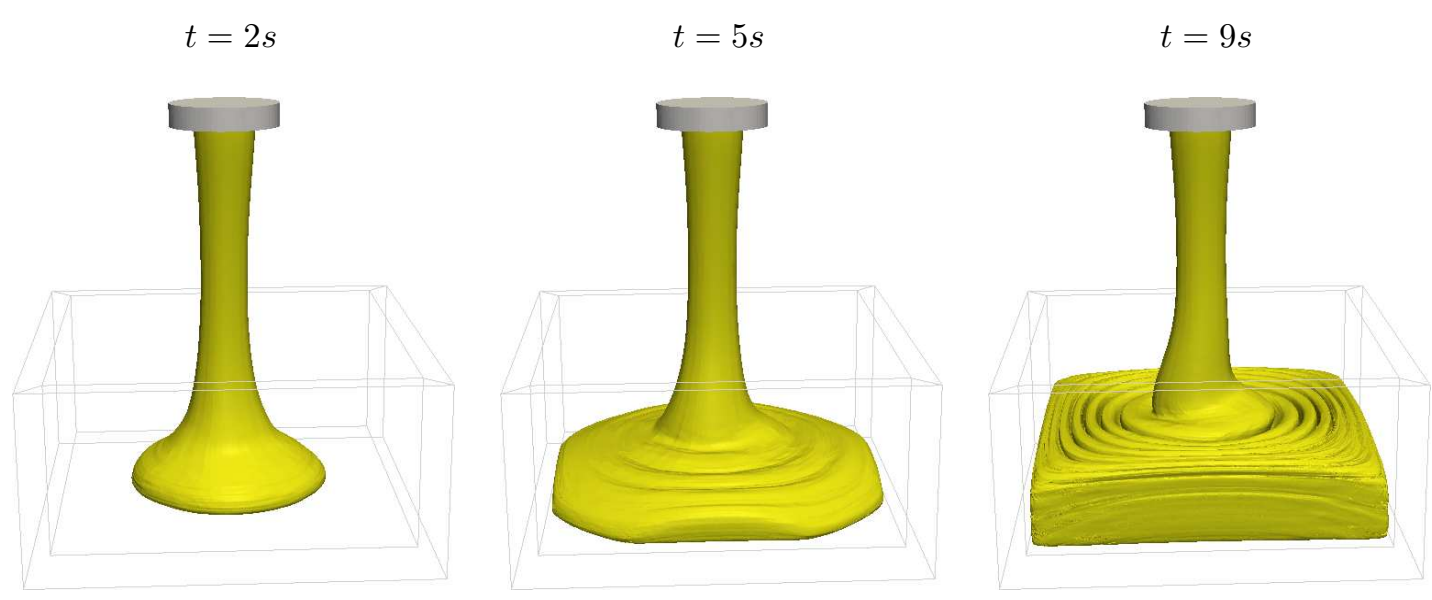

Figura 6.42: Modelo SXPP. Resultados obtidos com TDPUS-C3. $R e=0.64, W e=0.1, \varrho=0.5$, $\vartheta=0.9, \gamma=0.1, Q=1$.

$$
t=2 s
$$

$$
t=5 s
$$$$
t=9 \mathrm{~s}
$$
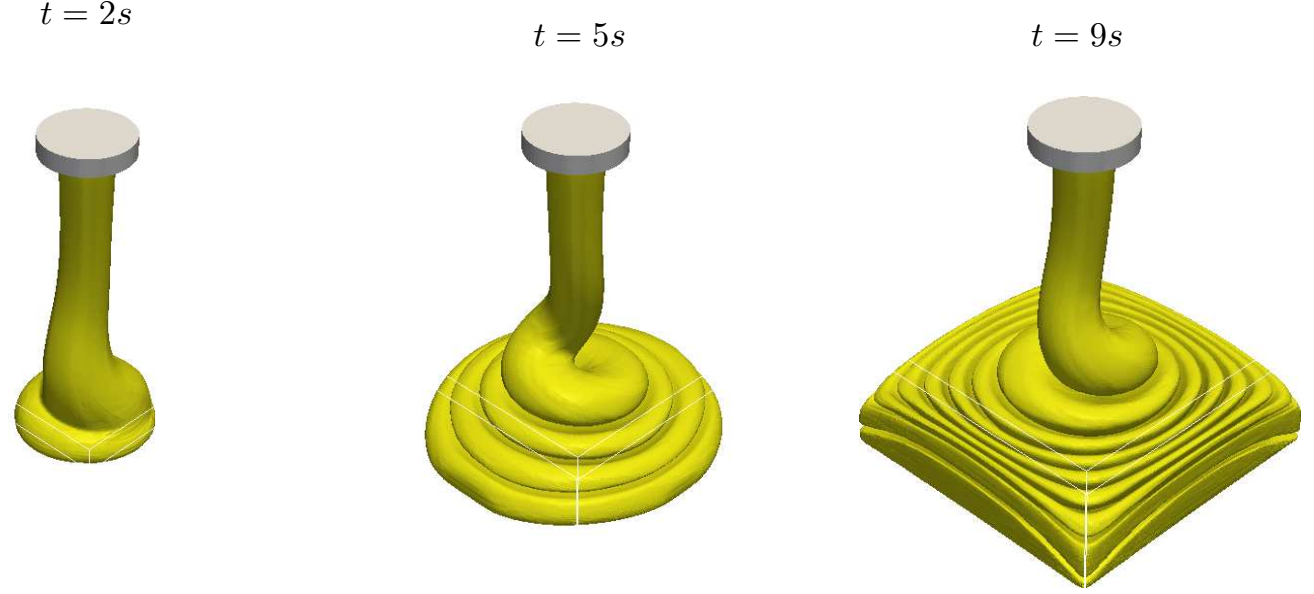

Figura 6.43: Modelo SXPP. Resultados obtidos com TDPUS-C3. $R e=0.32, W e=0.1, \varrho=0.5$, $\vartheta=0.9, \gamma=0.1$. 


$$
t=2 s
$$

$$
t=5 s
$$

$t=9 s$
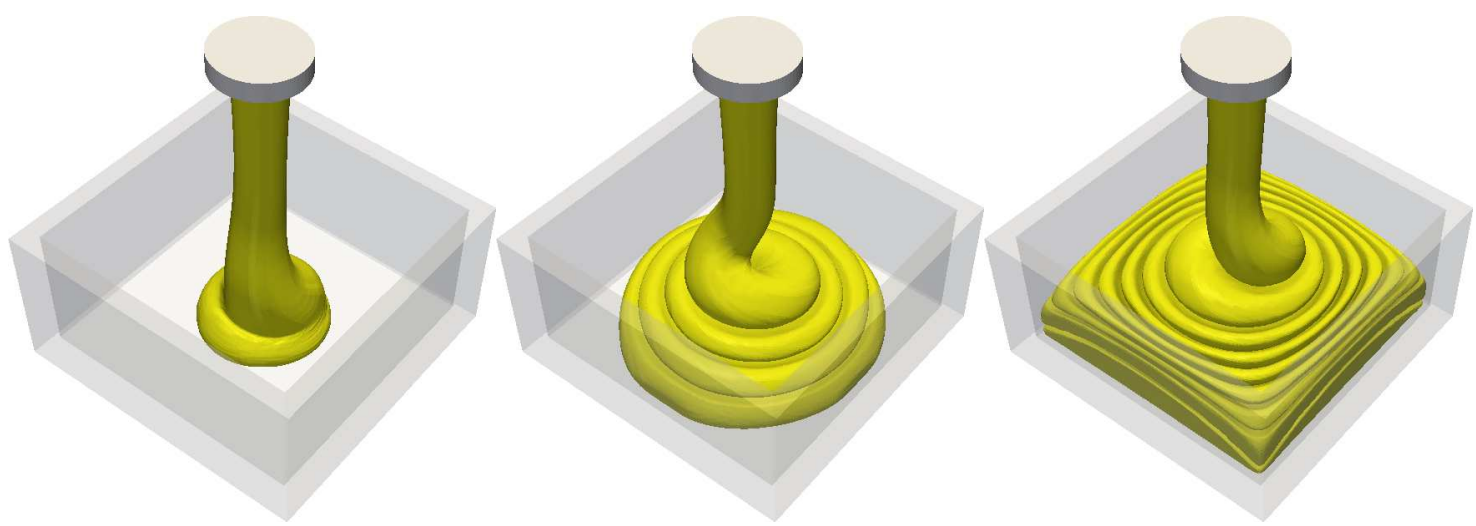

Figura 6.44: Modelo SXPP (Extraído de Figueiredo (2011)). Resultados obtidos com CUBISTA. $R e=0.32, W e=0.1, \varrho=0.5, \vartheta=0.9, \gamma=0.1$ e $Q=1$. 


\section{Capítulo}

\section{Considerações finais e trabalhos futuros}

O tema desta tese foi desenvolver, analisar e implementar um novo esquema convectivo tipo upwind, denominado TDPUS-C3 - dependente de um parâmetro livre $\beta$, para resolver sistemas hiperbólicos gerais. Buscou-se também implementar a associação deste novo esquema com as modelagens de turbulência $\kappa-\varepsilon$ padrão e $R N G \kappa-\varepsilon$ com o intuito de simular escoamentos incompressíveis turbulentos envolvendo superfícies livres móveis.

O esquema TDPUS-C3 foi derivado da aplicação dos critérios de estabilidade CBC e TVD, combinado com as condições de Leonard (1988a), e mostrou possuir três características importantes: simplicidade, robustez e generalidade de aplicação. Selecionando o parâmetro livre $\beta=567.25$, o esquema TDPUS-C3 mostrou introduzir pouca viscosidade numérica e não gerar oscilações, e com este valor de $\beta$ o desempenho do esquema foi avaliado resolvendo uma variedade de problemas, desde advecção de escalares até problemas de escoamentos complexos. Em particular, os resultados numéricos obtidos para o problema das instabilidades de Rayleigh-Taylor mostraram que o novo esquema é útil para resolver problemas envolvendo estruturas vorticais.

A associação das modelagens upwind TDPUS-C3 e $R N G \kappa-\varepsilon$ mostrou também ser útil na resolução numérica de escoamentos de fluido no regime turbulento com superfícies livres móveis. Em particular, comparações entre as soluções numéricas e analíticas para o problema do jato livre (2D e 3D) sobre uma superfície rígida foram realizadas, mostrando que as soluções obtidas com o modelo $\kappa-\varepsilon$ padrão são melhores quando comparadas com as soluções derivadas do modelo $R N G \kappa-\varepsilon$. Foi observado que o fluido espalha mais rápido com o uso da modelagem $R N G \kappa-\varepsilon$, motivado provavelmente pela pouca introdução de viscosidade numérica. Instabilidades numéricas foram detectadas também ao se simular o problema do jato livre 3D sobre uma superfície rígida impermeável em malhas finas, em que foram usados $\delta t$ inferiores ou iguais a $10^{-7}$. Devido a isto, projeta-se usar métodos implícitos para simular este problema com o objetivo de aumentar o passo temporal e, ao mesmo tempo, assegurar 
estabilidade. No caso do problema do colapso de fluido e interação da superfície livre com um obstáculo, a combinação $R N G \kappa-\varepsilon$-TDPUS-C3 foi um sucesso. Uma análise qualitativa dos resultados desta simulação mostraram estruturas turbilhonares (grandes vórtices) que não foram capturadas nos trabalhos de Raad e Bidoae (2005) e Kurokawa (2009).

Os estudos apresentados nesta tese estabelecem o potencial do esquema TDPUS-C3 e a utilidade da combinação deste esquema upwind com as modelagens da turbulência a duas equações $\kappa-\varepsilon$ padrão e $R N G \kappa-\varepsilon$ em resolver uma ampla gama de problemas complexos em DFC. Como continuação deste trabalho de pesquisa, projeta-se para o futuro realizar as seguintes atividades:

- simular as formas poligonais do salto hidráulico usando os efeitos da tensão superficial;

- implementar métodos implícitos para resolver escoamentos envolvendo turbulência;

- adaptar o novo esquema upwind TDPUS-C3 para malhas não estruturadas e adaptativas;

- associar o novo esquema convectivo às modelagens Realizable $\kappa-\varepsilon$ e LES;

- incrementar outros códigos (comerciais e abertos), tais como MFIX, OpenFOAM, CFX® e FLUENT®, com o esquema TDPUS-C3; 


\section{Produção científica associada}

Muitos dos resultados desta tese têm sido submetidos e/ou publicados em revistas e anais de congressos da área. Na sequência, apresenta-se um resumo de cada um deles.

\section{Artigos submetidos e publicados em revistas:}

- M.A.C. Candezano, L. Corrêa, G. A. B. de Lima and V. G. Ferreira, A comparative study of upwinding schemes applied to complex fluid dynamics equations. Applied Numerical Mathematics, 2012, 36 pág. (Submetido).

Resumo: The nonlinear Burgers, Buckley-Leverett and Euler equations are employed for a comparative study of the frequently used flux limiters, namely: the consecrated ARORA-ROE, van Albada, CUBISTA and ADBQUICKEST; and two recently introduced rational functions TOPUS and SDPUS-C1. The comparison is mainly done in terms of their abilities in resolving disconti- nuities and reproducing complex flow phenomena. Accuracy of the methods is also investigated on test problems for Burgers and Euler equations. It is concluded that both TOPUS and SDPUS-C1 can be considered as new alter native tools for computer simulations of fluid flow, with SDPUS-C1 the most favourable in treating convectively dominated problems. As application, the corresponding CBC-based SDPUS-C1 scheme is employed to simulate incompressible flows with moving free surfaces.

- L. Corrêa, G. A. B. Lima, M.A.C. Candezano, M. P. S. Braun, C. M. Oishi, H. A. Navarro and Valdemir G. Ferreira, A useful high-resolution convection upwind scheme for computational fluid dynamics algorithms. International Journal for Numerical Methods in Fluids, V. 31 (3), 2012, pág. 1 -26.

Resumo: A bounded upwinding scheme for numerical solution of hyperbolic conservation laws and Navier-Stokes equations is presented. The scheme is based on CBC and TVD stability criteria and developed by employing continuously differentiable functions. The accuracy of the scheme in space and time is verified by assessing the error on $1 D$ benchmark test cases. A comparative study between the 
new scheme and conventional upwind-biased schemes to solve standard nonlinear hyperbolic conservation laws is also accomplished. The scheme is then examined in the simulation of Newtonian and non-Newtonian fluid flows of increasing complexity; satisfactory agreement is found in terms of the overall behavior. Finally, the scheme is used to study hydrodynamics of a gas-solid flow in a bubbling fluidized bed.

- V.G. Ferreira, R. A. B. Queiroz, M.A.C. Candezano, G. A. B. Lima, L. Corrêa, C. O. Oishi, F. P. Santos, Simulation results and application of an advection bounded scheme to practical flows, Computational \& Applied Mathematics, 2012, 27 pág. (Aceito).

Resumo: This paper reports experiments on the use of a recently introduced advection bounded upwinding scheme, namely TOPUS (Computers 63 Fluids 57 (2012) 208-224), for flows of practical interest. The numerical results are compared against analytical, numerical and experimental data and show good agreement with them. It is concluded that the TOPUS scheme is a competent, powerful and generic scheme for complex flow phenomena.

- G.A.B. de Lima, V.G. Ferreira, E.R. Cirilo, A. Castelo, M.A.C. Candezano, I.V.M. Tasso, D.M.C. Sano , L.V.A. Scalvi, A continuously differentiable upwinding scheme for the simulation of fluid flow problems. Applied Mathematics and Computation, 2012, V. 218 (7), pág. 8614-8633.

Resumo: This paper deals with the numerical solution of complex fluid dynamics problems using a new bounded high resolution upwind scheme (called SDPUS-C1 henceforth), for convection term discretization. The scheme is based on TVD and CBC stability criteria and is implemented in the context of the finite volume/difference methodologies, either into the CLAWPACK software package for compressible flows or in the Freeflow simulation system for incompressible viscous flows. The performance of the proposed upwind non-oscillatory scheme is demonstrated by solving two-dimensional compressible flow problems, such as shock wave propagation and two-dimensional/axisymmetric incompressible moving free surface flows. The numerical results demonstrate that this new cell-interface reconstruction technique works very well in several practical applications.

- Valdemir Garcia Ferreira, Giseli Aparecida Braz de Lima, Laís Corrêa, M. A. C.Candezano, Eliandro Rodrigues Cirilo; Paulo Laerte Natti e Neyva Maria Lopes Romeiro, Computational evaluation of convection schemes in fluid dynamics problems. Semina: Ciências Exatas e Tecnológicas, UEL, Londrina, 2011, 8 pág.

Resumo: This article provides a computational evaluation of the popular high resolution upwind WACEB, CUBISTA and ADBQUICKEST schemes for solving non-linear fluid dynamics problems. By using the finite difference methodology, the schemes are analyzed and implemented in the context of normalized variables of Leonard. In order to access the performance of the schemes, Riemann problems for $1 D$ Burgers, Euler and shallow water equations are considered. From the numerical results, the schemes are ranked according to their performance in solving these non-linear equations. The best scheme is then applied in the numerical simulation of tridimensional incompressible moving free surface flows. 


\section{Artigos publicados em anais de congressos:}

- M.A.C. Candezano, Valdemir G. Ferreira, Implementation of upwinding schemes for solving the double Mach reflection problem. XXXIV Congresso Nacional de Matemática Aplicada, CNMAC 2012, 5 pág.

Resumo: An upwind implementation for solving the double Mach reflection problem using two recently developed and three well known TVD schemes is presented. We show that the recently developed TVD high-resolution polynomial upwind schemes are able to capture the complex (vortical) structures, specially of the density contours, in the "blown up" region. The numerical results are in agreement with the original studies of Woodward and Colella (1984) and are comparable with those computed with the ENO/WENO schemes.

- M. A. C. Candezano, Valdemir G. Ferreira, G.A. B. de Lima, A new type of $T V D / C B C$ polynomial upwind scheme for hyperbolic conservation laws and fluid dynamics problems. XIV Brazilian Congress of Thermal Sciences and Engineering, ENCIT 2012, 8 pág.

Resumo: A new high-resolution polynomial TVD/CBC-based upwind scheme is developed for numerical solution of hyperbolic conservation laws and related fluid dynamic problems. The scheme, called TDPUS-C3, is implemented into the CLAWPACK software. Unsteady simulation of nonlinear problems demonstrates that the scheme is capable of stably reproducing shocks, discontinuities and complex structures in flows.

- Valdemir G. Ferreira, Rodolfo Perez, M.A.C. Candezano, Numerical simulation of complex problems in fluid dynamics. VII Congresso Nacional de Engenharia Mecânica, CONEM 2012, 10 pág. Resumo: This article is concerned with the numerical simulation of complex problems in fluid dynamics using a recently introduced convection upwind scheme. The scheme is developed based on Hermite interpolation and CBC/TVD stability criteria; it is implemented in the context of finite volume/finite difference and into two simulation environment, namely: CLAWPACK, for 1D/2D systems of hyperbolic conservation laws; and Freeflow, for 3D incompressible Newtonian flows. The performance of the scheme is investigated in solving transport of scalars, non-linear Burguers and Euler equations. Comparisons with the results provided by the well established van Albada scheme are also presented. As application, the scheme is then employed to simulate 3D moving free surface Newtonian flows (broken dam and circular hydraulic jump). The results demonstrate that the upwind scheme discussed in this article works well in several PDEs of fluid dynamics.

- M.A.C. Candezano, Patricia Sartori, Laís Corrêa, Giseli A.B. de Lima, Rodolfo Perez, Valdemir G. Ferreira, A numerical study of some recently introduced TVD upwinding schemes with applications in fluid dynamics problems. 21st International Congress of Mechanical Engineering COBEM 2011, 9 pág.

Resumo: An important issue in computational fluid dynamics is the appropriate approximation of the convection phenomena. For this, the TVD schemes are alternatives to the ENO/WENO techniques due to the robustness, low cost and simplicity of implementation. Within this scenario, the aim of this our 
work is to present a numerical study of some recently introduced polynomial TVD upwinding schemes - namely TOPUS and SDPUS-C1 with applications in fluid dynamics problems. By using these new upwind schemes, numerical results for nonconvex nonlinear problem, 1D Euler equations, 2D advection of scalars and $2 D$ MHD equations are presented. Comparison with the well recognized CFL-dependent ARORA-ROE and ADBQUICKEST schemes and the conventional SUPERBEE and MC schemes are assessed. The TOPUS and SDPUS-C1 upwind schemes are developed in the context of normalized variables (NV) of Leonard and satisfy TVD constraints of Harten.

- André Luiz Andrade Simões, M.A.C. Candezano, Harry Edmar Schulz, Rodrigo de Melo Porto \& Valdemir G. Ferreira, Comparação de esquemas numéricos para as equações de Saint-Venant usando códigos livres. XIX Simpósio Brasileiro de Recursos Hídricos, 2011, pág. 19.

Resumo: Escoamentos em grandes escalas e em alguns problemas práticos são tratados normalmente por meio de sistemas de equações mais simples do que as equações gerais originais, denominadas equações de Saint-Venant ou equações para águas rasas. Este trabalho apresenta resultados de testes numéricos para as equações de Saint-Venant obtidos com quatro esquemas de alta resolução e dois esquemas clássicos de primeira e segunda ordens, todos calculados com códigos livres. A qualidade superior dos resultados obtidos com os métodos de alta resolução é demonstrada com o uso de malhas com diferentes refinamentos juntamente com comparações entre as soluções analíticas e dados numéricos da ruptura de uma barragem e da formação de ressalto hidráulico.

- M.A.C. Candezano, L. Corrêa, V. G. Ferreira, Application of new polynomial upwind schemes to Rayleigh-Taylor instability. Congresso de Matemática Aplicada e Computacional CMAC-SE 2011, 4 pág.

Resumo: We present in this work numerical solutions of the representative Rayleigh-Taylor instability problem. For this, we will use four high-resolution TVD upwind schemes. We will demonstrate that two recently developed TVD polynomial upwind schemes are able to compute this complex flow free from spurious oscillations and with small smearing. We conclude that the polynomial upwind schemes present, in coarse meshes, better resolution than two conventional upwind schemes.

- M.A.C. Candezano, Rodolfo Pérez, Juliana Bertoco \& Valdemir G. Ferreira, Três novos limitadores de fluxo para sistemas de leis de conservação, Encontro Nacional de Análise Matemática e Aplicações, V ENAMA, 2011, 2 pág.

Resumo: Muitos problemas em física e em engenharia são modelados por leis de conservação hiperbólicas, cujos termos não lineares (convectivos) influenciam sobremaneira a solução. A busca por um limitador de fluxo simples que aproxime bem esses termos e introduza pouca dissipação numérica, sem a presença de oscilações não-físicas, tem sido um desafio permanente para a comunidade científica moderna em dinâmica dos fluidos computacional. Vários limitadores de fluxo têm sido desenvolvidos nas últimas decadas, e dentre eles destaca-se o de Van Albada. Nesse trabalho, três novos limitadores de fluxo são apresentados, a saber: o HERMITE - baseado no polinômio interpolador de Hermite; o SPLINE - baseado na interpolação spline cúbica; e LIM-TESTE (limitador teste). 
- M. A. C. Candezano, Patricia Sartori, André Luiz Andrade Simões, Valdemir G. Ferreira, Numerical solutions of shallow water equations with source term using high-resolution bounded upwind schemes. Dincom'11, 10th Brazilian Conference on Dynamics, Control and their Applications, 2011, 4 pág.

Resumo: This paper addresses the problem of obtaining numerical solutions of shallow water equations with source term when flux limiters (ARORA-ROE, ADBQUICKEST, TOPUS, SUPERBEE and MINMOD) in upwind schemes are used. The numerical results show that these high-resolution upwind schemes provide better results than those obtained by VFRoe solver of Bouchut (Nonlinear Stability of Finite Volume Methods for Hyperbolic Conservation Laws and Well-Balanced Schemes for Sources, 2000).

- M.A.C. Candezano, P. Sartori, V.G. Ferreira, An upwind implementation of some conservation laws. Dincom'10, 9th Brazilian Conference on Dynamics, Control and their Applications, 2010, 9 pág. Resumo: This work provides a computational assessment of three high-resolution upwind schemes namely ADBQUICKEST, TOPUS and CUBISTA for solving some fluid dynamics problems. These schemes are developed in the context of the normalized variables $(\boldsymbol{N} \boldsymbol{V})$ of Leonard and in $\boldsymbol{T} \boldsymbol{V} \boldsymbol{D}$ constraints of Harten, and implemented using the finite difference methodology. We consider the application of these schemes to systems of conservation laws, $1 D$ and $2 D$, to demonstrate their performance in modeling such problems.

- M.A.C. Candezano, V. G. Ferreira. A comparative study of upwind differencing schemes in the context of schock capturing properties. XXXIII Congresso Nacional de Matemática Aplicada, 2010, 7 pág.

Resumo: This article provides a computational assessment of three well known high-resolution upwind schemes, namely WACEB ,CUBISTA and ADBQUICKEST, for solving fluid dynamics problems. In the context of the finite differences methodology, the schemes are analyzed and implemented in normalized variables of Leonard. The performance of these schemes is evaluated in solving Riemann problems for the Burgers, Euler and shallow water equations. The schemes are then ranked and the selected one is applied in the numerical simulation of $2 D$ magnetohydrodynamics equations.

- M.A.C. Candezano, P. Sartori, L. Corrêa, V.G. Ferreira, A computational evaluation of two high-resolution convective schemes for problems in fluid dynamics. XIII Brazilian Congress of Thermal Sciences and Engineering, 2010, 10 pág.

Resumo: It is well recognized that researchers face many problems for numerically approximating nonlinear convective terms in conservation laws and related fluid dynamics problems. One of the main challenges is to develop upwind schemes that capture well discontinuities (or shock waves) and allow high (at least second order) accuracy solution. In this scenario, the goal of this work is to present a computational evaluation of two genuinely Brazilian high resolution convective upwind schemes, namely ADBQUICKEST and SDPUS-C1, for solving general fluid dynamics problems. Both schemes are based in the context of normalized variables $(N V)$ of Leonard and satisfy the total-variation diminishing (TVD) constraints of Harten. 
- G.A.B. Lima, M.A.C. Candezano, L. Corrêa, V.G. Ferreira. Numerical simulation of Newtonian and non-newtonian free surface flows using the SDPUS-C1 upwinding scheme. XIV Brazilian Congress of Thermal Sciences and Engineering, ENCIT 2012, 2012, 8 pág.

Resumo: The study deals with the numerical simulation of complex incompressible fluid flows using a new continuously differentiable bounded convection scheme (called SDPUS-C1). The scheme is based on TVD stability criteria and implemented in the context of finite difference methodology. The performance of the SDPUS-C1 scheme is assessed by solving 2D Newtonian and non-Newtonian moving free surface flows.

- G.A.B. Lima, L. Corrêa, M.A.C. Candezano, P. Sartori, V.G. Ferreira. A Simple NVD/TVD-Based upwinding scheme for convection term discretization. V European Conference on Computational Fluid Dynamics. ECCOMAS CFD 2010, 2010, 18 pág.

Resumo: The correct modeling for processes involving convection, without introducing excessive artificial damping while retaining high accuracy, stability, boundedness and simplicity of implementation continues being nowadays a challenging task for the scientific CFD community. In this context, the objective of this study is to present and to evaluate the performance of a new TVD-based upwinding scheme, namely Six-Degree Polynomial Upwind Scheme of C 1 Class (SDPUS-C1), for convection term discretization. SDPUS-C1 satisfies the TVD principle of Harten and is based on the NVD formulation of Leonard. Firstly, a description of the scheme is done and then numerical results are presented for two-dimensional hyperbolic conservation laws, such as acoustics, Burgers and Euler equations. Finally, as application, the SDPUS-C1 scheme is used for the computational simulation of three-dimensional incompressible fluid flows involving moving free surfaces.

- P. Sartori, G.A.B. Lima, L. Corrêa, M.A.C.Candezano, V.G. Ferreira. Avaliação Computacional de Três Esquemas Upwind Originais. $9^{0}$ SIMPÓSIO DE MECÂNICA COMPUTACIONAL, 2010, 9 pág.

Resumo: Resolver numericamente problemas em dinâmica dos fluidos é uma tarefa difícil e desafiadora,principalmente quando tais problemas são dominados por convecção. Isso requer o desenvolvimento de esquemas numéricos tipo upwind que sejam precisos, monotônicos e robustos. O presente trabalho é destinado à avaliação computacional de três novos esquemas upwind de alta resolução, desenvolvidos no LCAD-ICMC/USP, denominados ADBQUICKEST,TOPUS e SDPUS-C1. O desempenho desses esquemas é investigado a partir da simulação computacional de leis de conservação hiperbólicas, a saber: advecção de escalares e problemas de Riemann para acústica e equações de Euler da dinâmica dos gases. E então, como aplicação, esses esquemas são utilizados na simulação de escoamentos incompressíveis com superfícies livres móveis modelados pelas equações de Navier-Stokes 3D.

- G.A.B. Lima, M.A.C. Candezano, V.G. Ferreira, Uma Avaliação computacional de três esquemas de discretização upwind para leis de conservação não lineares. Dincom'08, 8th Brazilian Conference on Dynamics, Control and their Applications, 2009, 9 pág.

Resumo: Neste trabalho de pesquisa é apresentada uma avaliação computacional dos esquemas de 
alta resolução WACEB, CUBISTA e ADBQUICKEST para resolver leis de conservação lineares e não lineares. Utilizando-se a metodologia de diferenças finitas, esses esquemas são analisados e implementados no contexto de variáveis normalizadas de Leonard. Para acessar o desempenho dos esquemas, quatro problemas (um linear e três não lineares) são considerados, a saber: problema de advecção de um escalar; e problemas de Riemann para as equações de Burgers, Euler e águas rasas.

- G.A.B. Lima, V.G. Ferreira, R.A.B. Queiroz, M.A.C. Candezano, L. Corrêa, Development and evaluation of upwind schemes for conservation laws. 20o. Congresso Brasileiro de Engenharia Mecânica COBEM2009, 2009, 10 pág.

Resumo: In the present work, it is presented a computational evaluation of two new high resolution upwind schemes, namely ADBQUICKEST and TOPUS, for solving general conservation laws. By using the finite difference methodology, these schemes are analyzed and implemented in the context of normalized variables of Leonard (NVD). In order to access the performance of the schemes, five test problems are simulated, namely, advection of a scalar, Riemann problems for Burgers, Buckley-Leverett, shallow water, and Euler equations. As applications, the schemes are used in the numerical simulation of incompressible Navier-Stokes equations. 


\section{Código para solução da equação de Burgers 2D utilizando a linguagem}

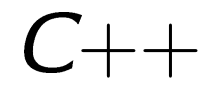

Listing B.1: Burgers2D.cpp

\#include $<$ iostream $>$

\#include $<$ stdlib.h $>$

\#include $<$ stdio.h $>$

\#include $<$ math . $\mathrm{h}>$

\#include $<$ ctime $>$

\#define pi 3.14159265358979

\#define eps $1 . e-8$

double TDPUS_C3_CONV_i (double $* * u$, double dt, double dx, double CFL, double delta, int i, int j, int N); double TDPUS_C3_CONV_j(double $* * u$, double dt, double dy, double CFL, double delta, int i, int $j$, int M); void escreve (double $* * \mathrm{u}$, double $* \mathrm{x}$, double $*$ y, double $\mathrm{t}$, double delta, double CFL, int $\mathrm{N}$, int $\mathrm{M}$, int caso); void inicializa (double $* * \mathrm{u}$, double $* x$, double $* y$, int $\mathrm{N}$, int $\mathrm{M}$ );

void contorno(double $* * u$, int $\mathrm{N}$, int $\mathrm{M}$, int o);

double $\mathrm{f}$ (double $* * \mathrm{u}$, int $\mathrm{i}$, int $\mathrm{j}$ );

double $\mathrm{g}$ (double $* * \mathrm{u}$, int $\mathrm{i}$, int $\mathrm{j})$;

double ubarra_i( double $* * u$, int $\mathrm{i}$, int $\mathrm{j}$, int $\mathrm{o}$, double $\mathrm{dt}$, double $\mathrm{dx}$ );

double ubarra_j( double $* * u$, int i, int $\mathrm{j}$, int o, double dt, double dy);

using namespace std;

int main (int $\operatorname{argc}$, char $* \operatorname{argv}[])$

\{

int i , j, k, N,M,NN, L, l, caso ;

double $\mathrm{a}=0.0, \mathrm{~b}=0.0, \mathrm{c}=0.0, \mathrm{~d}=0.0, \mathrm{dx}=0.0, \mathrm{dy}=0.0, \mathrm{dt}=0.0, \mathrm{t}=0.0, \mathrm{CFL}=0.0, \mathrm{delta}=0.0$;

double $\mathrm{tf}=0.0$;

double aux $=0.0$;

double delt $[1]$;

$\mathrm{tf}=0.5 / \mathrm{pi}$

$/ /$ tempo final

$\mathrm{N}=\operatorname{atoi}(\operatorname{argv}[1])$;

//malha em $x$ 


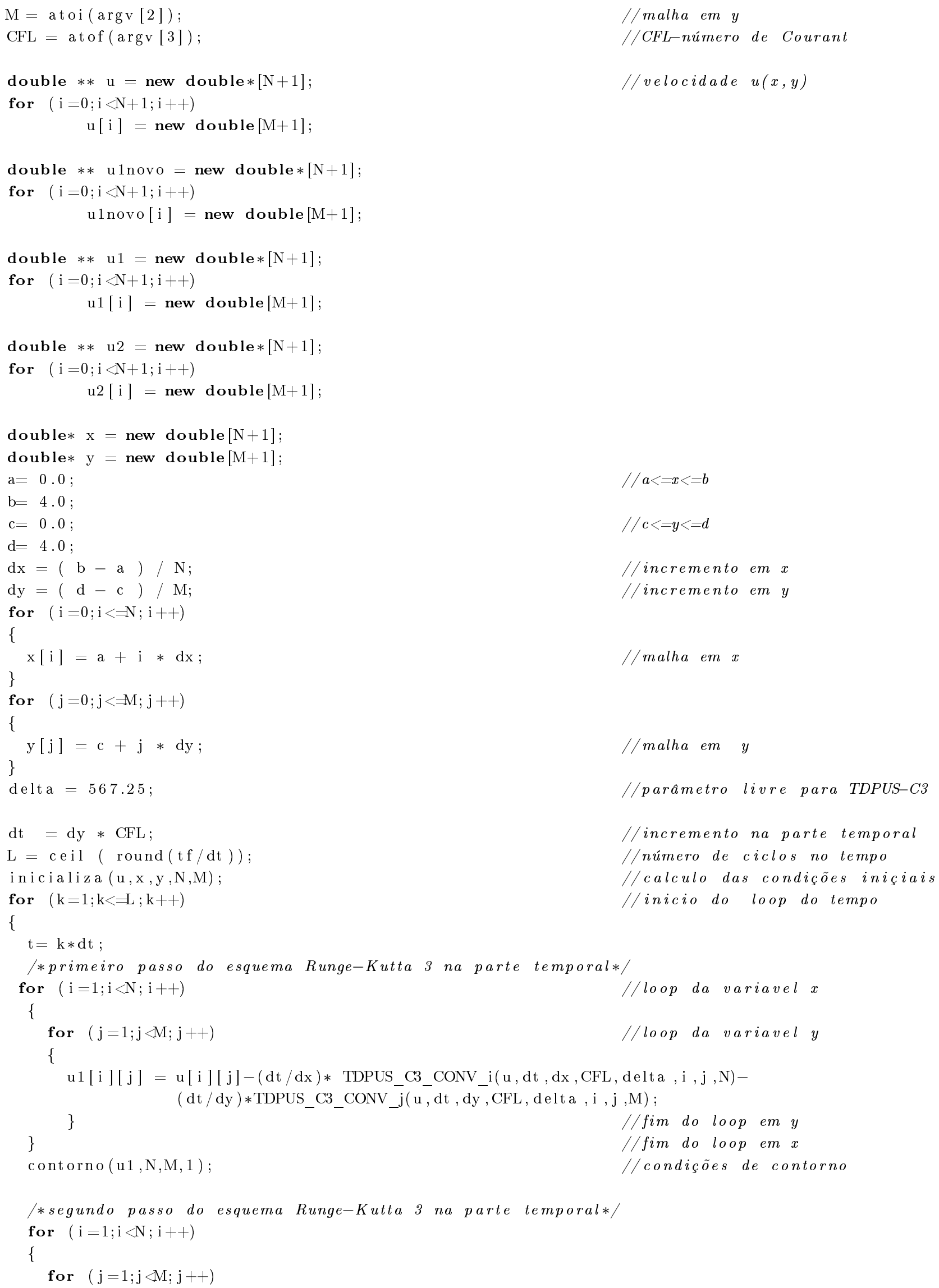




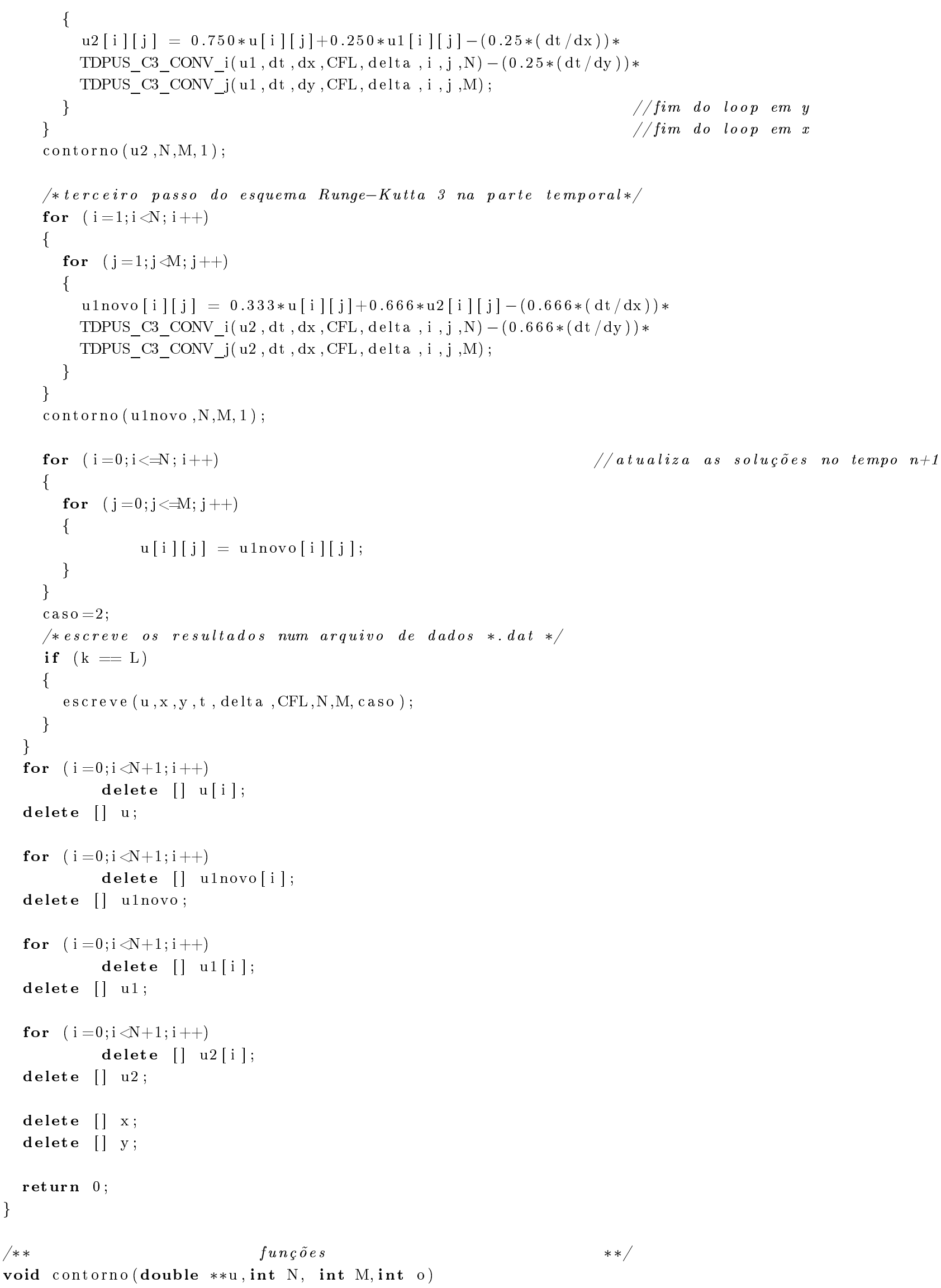




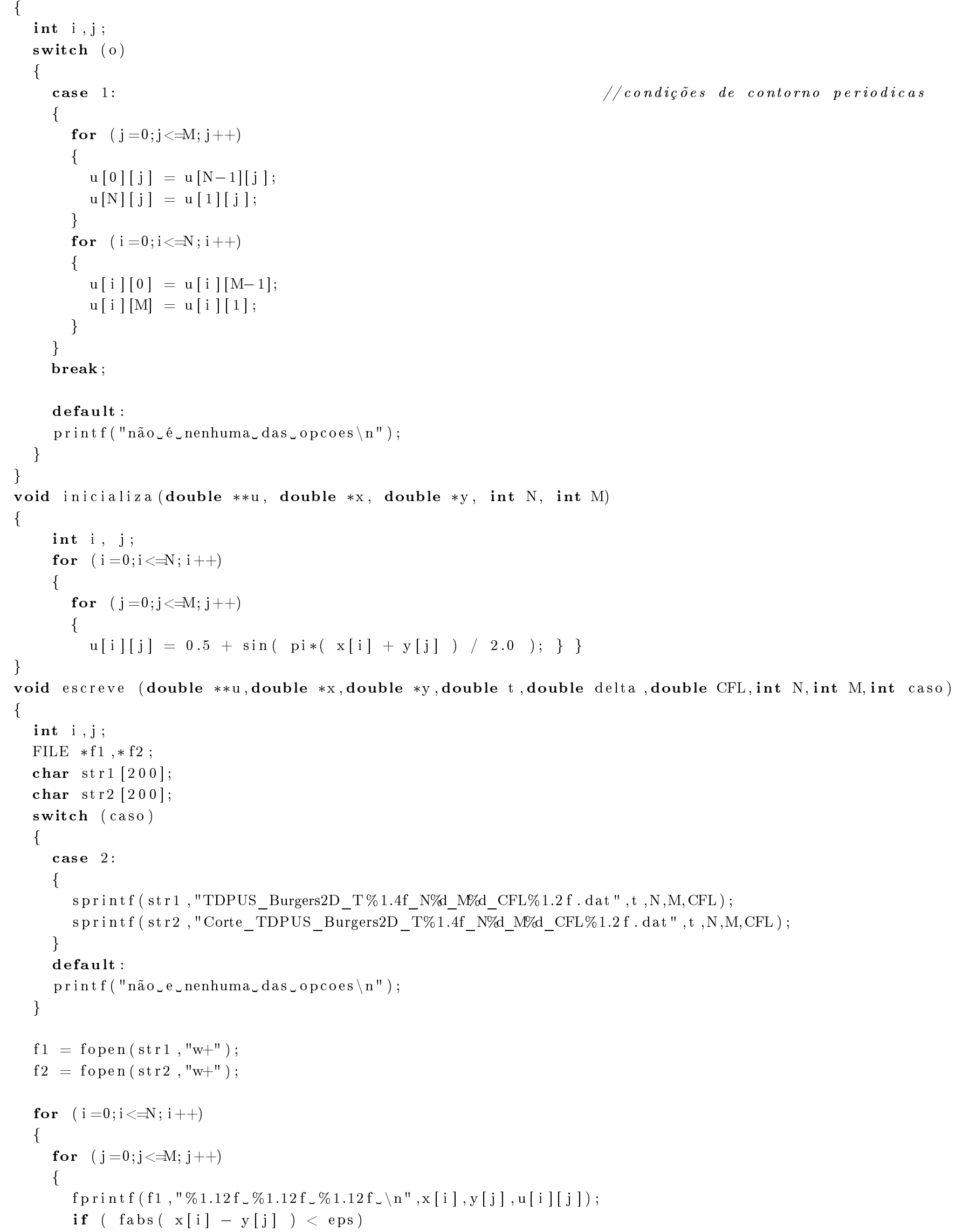




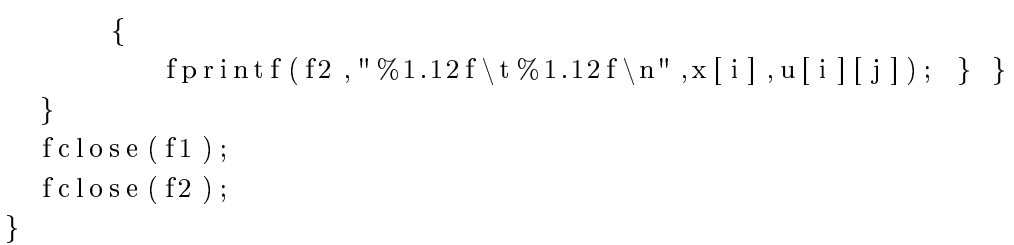




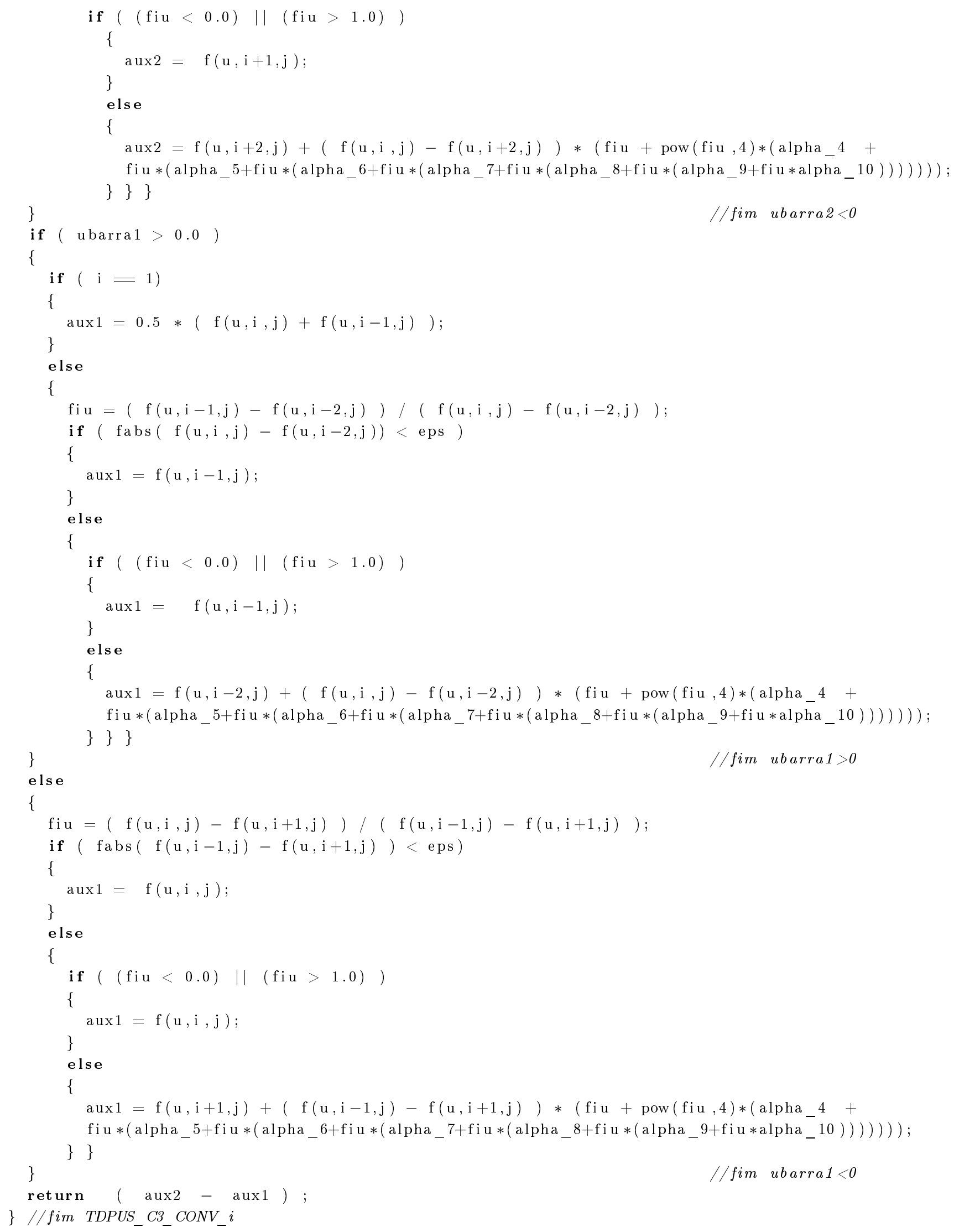


\{

double aux $2=0.0, \operatorname{aux} 1=0.0 ;$

double fiu $=0.0$;

double ubarra $2=0.0$, ubarra $1=0.0 ;$

double alpha_3, alpha_4, alpha_5, alpha_6, alpha_7, alpha_8, alpha_9, alpha_10;

alpha_ $10=4.0 *$ delta -384.0 ;

alpha_9 $=1856.0-20.0 *$ delta;

alpha_8 $=41.0 *$ delta -3584 ;

alpha_ $7=3456.0-44.0 *$ delta;

alpha $6=26.0 *$ delta $-1664.0 ;$

alpha_5 $=320.0-8.0 *$ delta;

alpha_ $4=$ delta;

alpha_ $3=1.0$;

ubarra $2=$ ubarra_j(u, i, j, 2, dt , dy );

ubarra1 = ubarra_j(u, i, j, 1, dt , dy $)$;

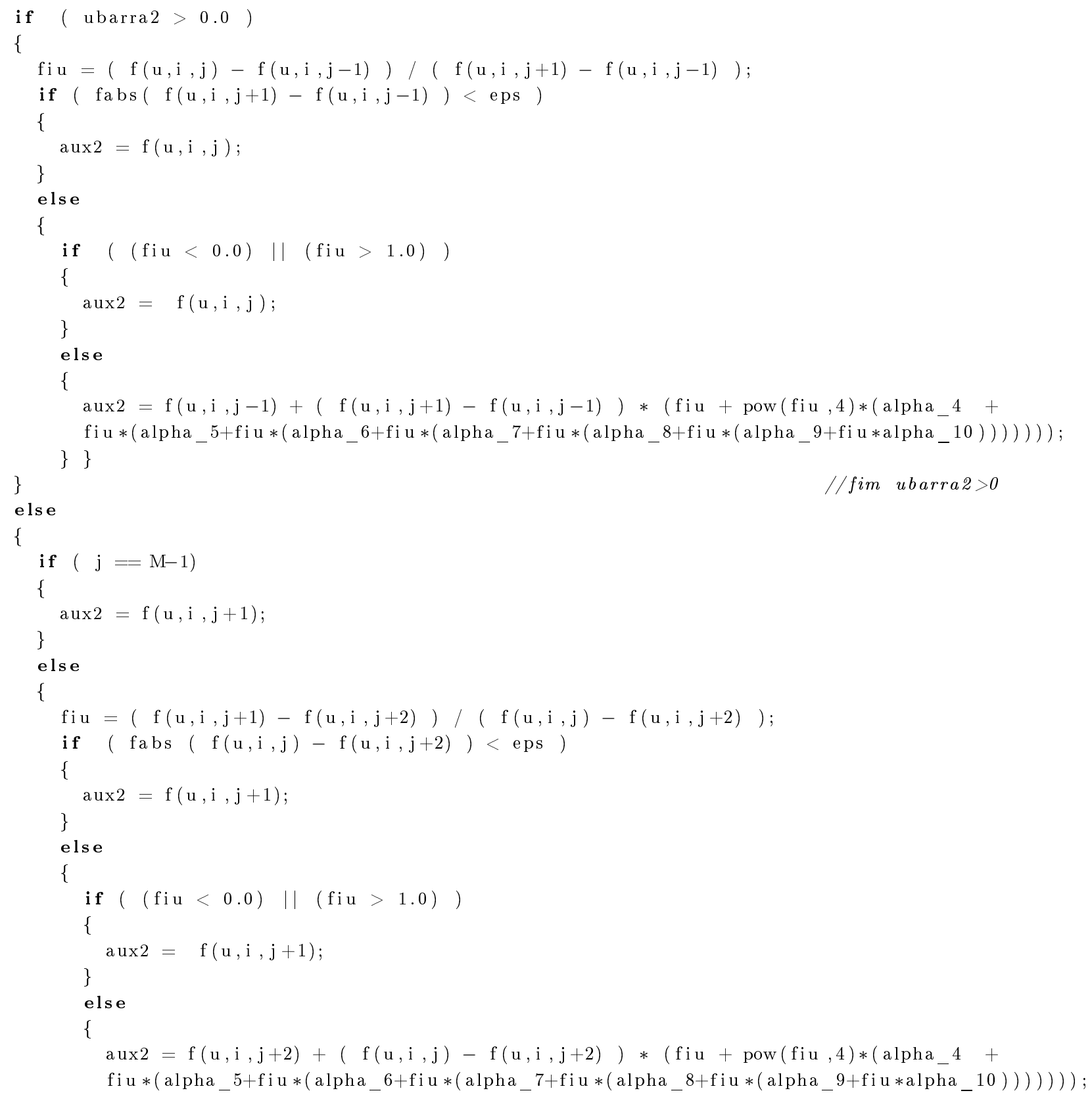




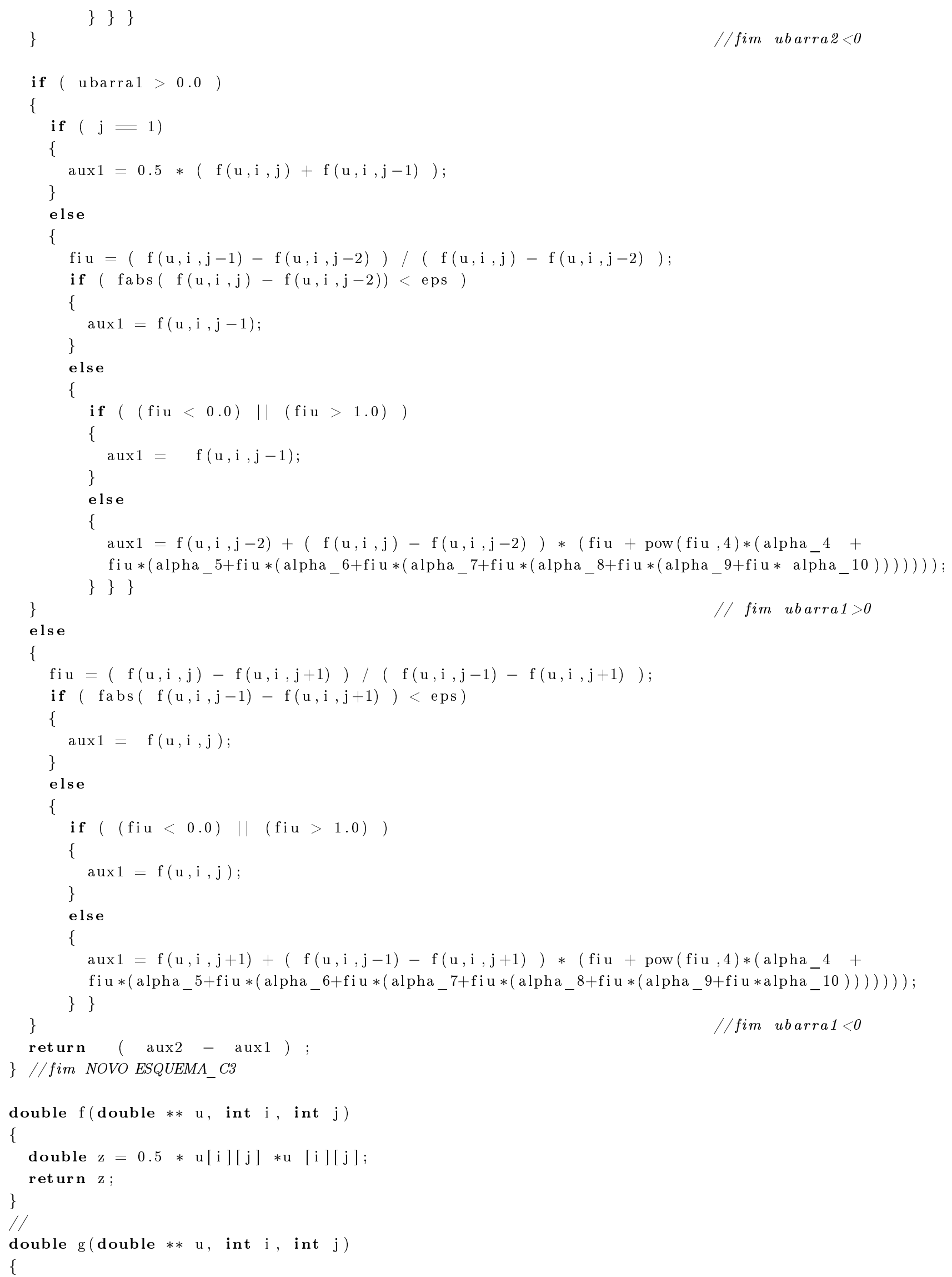




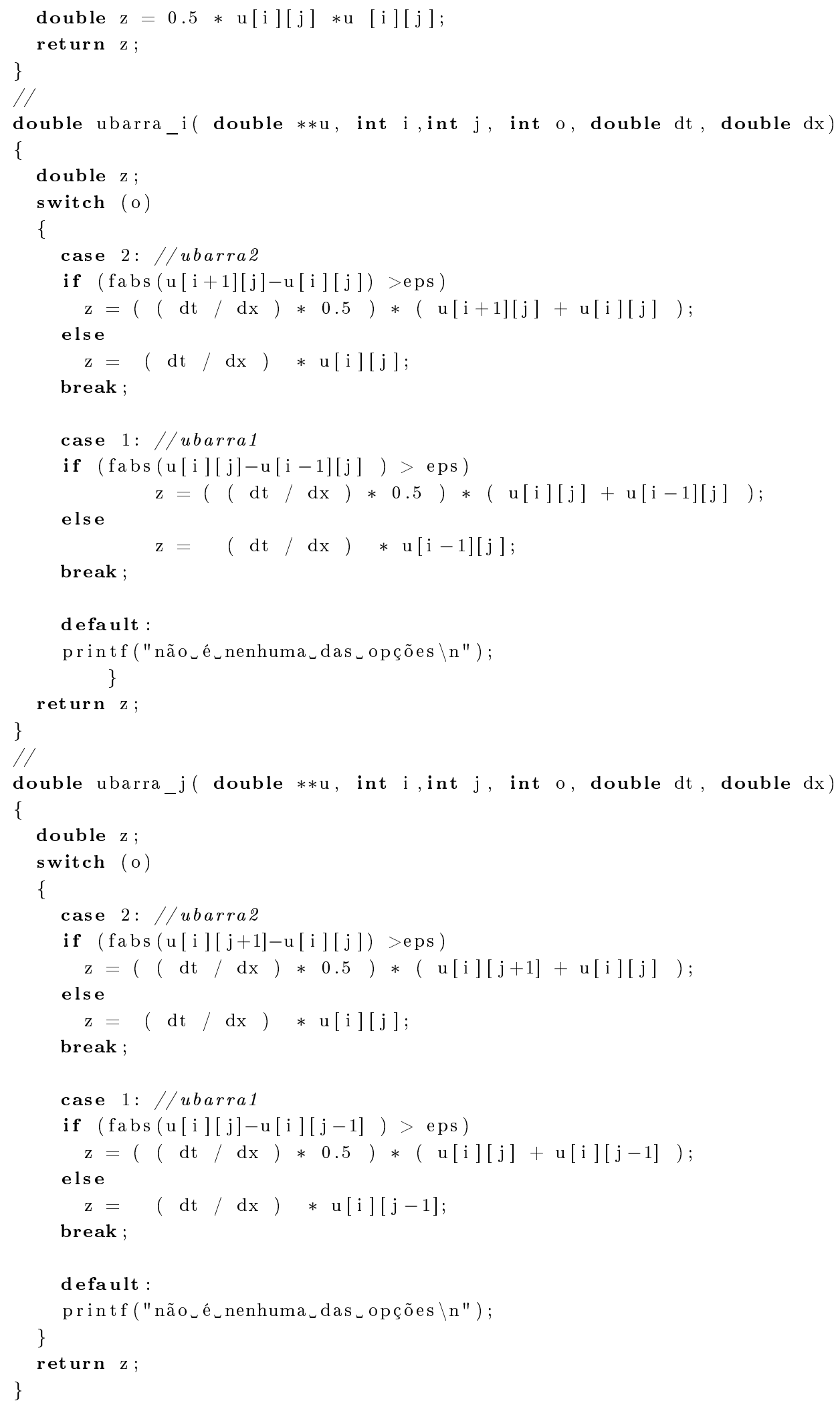





\section{Referências Bibliográficas}

Ahmed, R. (2004). Numerical schemes applied to the Burgers and Buckley-Leverett equations. Dissertação de Mestrado, University of Reading.

Alves, M. A., Oliveira, P. J., e Pinho, F. T. (2003). A convergent and universally bounded interpolation scheme for the treatment of advection. Int. J. Numer. Methods Fluids, 41:47-75.

Alves, M. A., Pinho, F. T., e Oliveira, P. J. (2000). Effect of a high-resolution differencing scheme on finite-volume predictions of viscoelastic flows. J. Non-Newtonian Fluid Mech., 93:287-314.

Amsden, A. A. e Harlow, F. H. (1970). A simplified MAC technique for incompressible fluid flow calculations. J. Comput. Phys., 6:332-335.

Arora, M. e Roe, P. (1997). A Well-Behaved TVD Limiter for High-Resolution Calculations of Unsteady Flow. J. Comput. Phys., 132:3-11.

Balbás, J., Tadmor, E., e Wu, C.-C. (2004). Non-oscillatory central schemes for one- and two-dimensional MHD equations: I. J. Comput. Phys., 201:261-285.

Barmin, A. A., Kulikowsky, A. G., e Pogorelov, N. V. (1996). Shock-Capturing Approach and Nonevolutionary Solutions in Magnetohydrodinamics. J. Comput. Phys., 126:77-90.

Baxevanou, C. A. e Fidaros, D. K. (2008). Validation of numerical schemes and turbulence models combinations for transient flow around airfoil. Engineering Applications of Computational Fluid Mechanics, 2:208-221.

Bohr, T., Dimon, P., e Putkaradze, V. (1993). Shallow-water approach to the circular hydraulic jump. J. Fluid Mech., 254:635-648.

Boris, B. e Book, D. (1976). Solution of the continuity equation by the method of flux corrected transport. J. Comput. Phys., 16:85-129.

Boris, B. P. e Book, D. L. (1973). Flux corrected transport: I SHASTA, a fluid tansport algorithms that works. J. Comput. Phys., 11:38-69. 
Bouchut, F. (2000). Nonlinear Stability of Finite Volume Methods for Hyperbolic Conservation Laws and Well-Balanced Schemes for Sources. Birkhauser Verlag.

Bradshaw, P. (1976). Turbulence, Topics in Applied Physics V. 12. Springer-Verlag.

Bradshaw, P., editor (1995). The Law of the Wall in Turbulent Flow, volume 451, No.1941. Osborne Reynolds.

Brechet, Y. e Néda, Z. (1999). On the circular hydraulic jump. Am. J. Phys., 67 - 8:723-731.

Brio, M. e Wu, C. C. (1988). An upwind differencing scheme for the equations of ideal magnetohydrodynamics. J. Comput. Phys., 75:400-422.

Candezano, M. A. C., Sartori, P., Correa, L., Lima, G. A. B., e Ferreira, V. G. (2010a). A computational evaluation of two high-resolution convective schemes for problems in fluid dynamics. In 13th Brazilian Congress of Thermal Sciences and Engineering, ENCIT 2012. Uberlândia, MG. Brazil.

Candezano, M. A. C., Sartori, P., e Ferreira, V. G. (2010b). An upwind implementation of some conservation laws. In 9th Brazilian Conference on Dynamics, Control and their Applications - DINCOM'10, Águas de Lindóia, SP. Brazil.

Carvalho, D. M., Maciel, J. L., Ravarto, L. P., Garcia, R. E., Ferreira, V. G., e Scalvi, L. (2010). Numerical simulation of the liquid phase in $\mathrm{SnO} 2$ thin film deposition by sol-gel-dip-coating. $J$. Sol-Gel Sci. Technol., 55:385-393.

Castelo, A., Tomé, M., Cesar, C. N. L., MacKee, S., e Cuminato, J. A. (2000). Freeflow: an integrated simulation system for three-dimensional free surface flows. Comput. Vis. Sci., 2:199-210.

Castro, M., Costa, B., e Don, W. S. (2011). High order weighted essentially non-oscillatory WENO-Z schemes for hyperbolic conservation laws. J. Comput. Phys., 230:1766-1792.

Chandrasekhar, S. (1961). Hydrodynamic and Hydromagnetic Stability. Oxford University Press.

Choi, S. K., Nam, H. Y., e Cho, M. (1995). Evaluation of a higher-order bounded convection scheme:three dimensional numerical experimental. Numer. Heat Transfer. B, 28:23-28.

Chorin, A. J. (1968). Numerical solution of the Navier-Stokes equations. Math. Comput., 22:745-762.

Cockburn, B. e Shu, C. W. (2001). Runge-Kutta discontinuous Galerkin methods for convection-dominated problems. J. Sci. Comput., 16:173-261.

Colagrossi, A. e Landrini, M. (2003). Numerical simulation of interfacial flows by smoothed particle hydrodynamics. J. Comput. Phys., 191:448-475.

Corrêa, L., Lima, G. A. B., Candezano, M. A. C., Braun, M. P. S., Oishi, C. M., Navarro, H. A., e Ferreira, V. G. (2012). A useful high-resolution convection upwind scheme for computational fluid dynamics algorithms. Int. J. Numer. Methods Fluids, páginas 1-26. 
Corrêa, L., Lima, G. A. B., e Ferreira, V. G. (2010). A C2 class TVD-Based Upwinding Scheme for Approximating Convection Terms. In XXXIII Congresso Nacional de Matemática Aplicada e Computacional. CNMAC 2010. Águas de Lindóia, SP. Brazil.

Courant, R., Isaacson, E., e Rees, M. (1952). On the solution of nonlinear hyperbolic differential equations by finite differences. Commun. Pur. Appl. Math., 5:243-255.

Cruchaga, M. A., Celentano, D. J., e Tezduyar, T. E. (2007). Collapse of a liquid column: numerical simulation and experimental validation. Comput. Mech., 39:453-476.

Cruickshank, J. e Munson, B. (1982). The viscous-gravity jet in stagnation flow. Transactions of the ASME, 104:360-362.

Cruickshank, J. O. (1980). Viscous fluid buckling: a theoretical and experimental analysis with extensions to general fluid stability. Tese de Doutorado, Iowa State University.

Cruickshank, J. O. (1988). Low-Reynolds-number instabilities in stagnating jet flows. J. Fluid Mech., 193:111-127.

Cruickshank, J. O. e Munson, N. R. (1981). Viscous fluid buckling of plane and axisymmetric jets. Phys. Fluids, 113:221-239.

Dahlburg, R. B. e Picone, J. M. (1989). Evolution of the Orszag-Tang vortex system in a compressible medium. Initial average subsonic flow. Phys. Fluids B I, 1:2153.

Darvish, M. S. (1993). A new high-resolution scheme based on the normalized variable formulation. Numer. Heat Transfer. B, 24:357-373.

Debnath, L. (1989). Nonlinear Partial Differential Equations for Scientists and Engineers. Basile:Birkhauser.

Déspres, B. e Lagoutiere, F. (2001). Contact Discontinuity Capturing Schemes for Linear Advection and Compressible Gas Dynamics. J. Sci. Comput., 16:489-523.

Einfeldt, B. (1988). On Godunov-type methdos for gas dynamics. SIAM J. Numer. Anal., 25:294-318.

Elkaim, D., Reggio, M., e Camarero, R. (1992). Simulating two dimensional turbulent flow by using $\kappa-\varepsilon$ model and the vorticity-stream function. Int. J. Numer. Methods Fluids, 14:961-980.

Evans, C. e Hawley, J. F. (1988). Simulation of magnetohydrodynamic flow: a constrained transport method. Astrophys. J., 332:659.

Ferreira, V. G., Kaibara, M. K., Lima, G. A. B., Silva, J. M., Sabatini, M. H., Mancera, P. F. A., e McKee, S. (2013). Application of a bounded upwinding scheme to complex fluid dynamics problems. Mathematical and Computer Modelling, 57 (3-4):435-439.

Ferreira, V. G., Kurokawa, F. A., Queiroz, R. A. B., Kaibara, M. K., Oishi, C. M., Cuminato, J. A., Castelo, A., Tomé, M. F., e McKee, S. (2009). Assessment of a high-order finite difference upwind scheme for the simulation of convection-diffusion problems. Int. J. Numer. Methods Fluids, 60:1-26. 
Ferreira, V. G., Mangiavacchi, N., Tomé, M. F., Castelo, A., Cuminato, J. A., e McKee, S. (2004). Numerical simulation of turbulent free surface flow using two equation $\kappa-\varepsilon$ viscosity models. Int. J. Numer. Methods Fluids, 44:347-375.

Ferreira, V. G., Oishi, C. M., Kurokawa, F. A., Kaibara, M. K., Cuminato, J. A., Castelo, A., Tomé, M. F., e Mckee, S. (2007). A combination of implicit and adaptative upwind tools for the numerical solution of incompressible free surface flows. Commun. Numer. Meth. En., 23:419-445.

Ferreira, V. G., Queiroz, R. A. B., Lima, G. A. B., Cuenca, R. G., Oishi, C. M., Azevedo, J. L. F., e McKee, S. (2012). A bounded upwinding scheme for computing convection-dominated transport problems. Comput. Fluids, 57(0):208-224.

Figueiredo, R. A. (2011). Estudos numéricos para a simulação de escoamentos viscoelásticos com superfície livre. Dissertação de Mestrado, Instituto de Ciências Matemáticas e de Computação. USP.

Fudihara, T. J., Jr., L. G., e Mori, M. (2007). A numerical investigation of the aerodynamics of a furnace with a movable block burner. Brazilian Journal of Chemical Engineering, 24-2:233-248.

Gaskell, P. H. e Lau, A. K. C. (1988). Curvature-compensated convective transport: SMART, a new boundedness-preserving transport algorithm. Int. J. Numer. Methods Fluids, 8:617-641.

Ghadimi, M., Ghadamian, H., Hamidi, A., Fazelpour, F., e Behghadam, M. A. (2012). Analysis of free and forced convection in air flow windows using numerical simulation heat transfer. International Journal of Energy and Environmental Engineering, 3:14:1-25.

Godunov, S. K. (1959). A Difference Scheme for Numerical Solution of Discontinuous Solution of Hydrodynamic Equations. Mat. Sb., 49:151.

Gottlieb, S. e Chi-Wang-Shu (1998). Total variation diminishing Runge-Kutta schemes. Math. Comput., 67:73-85.

Gottlieb, S., Shu, C.-W., e Tadmor, E. (2001). Strong Stability-Preserving High-Order Time Discretization Methods. SIAM Rev., 43:89-112.

Goutal, N. e Maurel, F. (1997). Proceedings of the 2nd Workshop on Dam-Break Wave Simulation. Relatório técnico, Groupe Hydraulique Fluviale, Département Laboratoire National d'Hydraulique, Electricité de France.

Greenough, J. e Rider, W. (2004). A quantitative comparison of numerical methods for the compressible Euler equations: fifth-order WENO and piecewise-linear Godunov. J. Comput. Phys., 196:259-281.

H. Lee Norris, W. R. (1975). Turbulent channel flow with a moving wavy boundary. Relatório técnico, Stanford University.

Hansen, S. H., Zauner, S. H. D., Dimon, P., Ellegaard, C., e Creagh, S. C. (1997). Geometric orbits of surface waves from a circular hydraulic jump. Phys. Rev. E, 55:7048-7062. 
Harlow, F. H. e Welch, J. E. (1965). Numerical calculations of time dependent viscous. Phys. Fluids, 8:2182-2189.

Harten, A. (1983). High resolution schemes for hyperbolic conservation laws. J. Comput. Phys., 49:357-393.

Harten, A., Hyman, J., e Lax, P. (1976). On finite difference approximation and entropy conditions for shocks. Comm. Pure Appl. Math., 29:297-322.

Harten, A., Lax, P. D., e van Leer, B. (1983). On upstream differencing and Godunov-type schemes for hyperbolic conservation laws. SIAM Review, 25:35-61.

Hirsch, C. (2007). Numerical Computation of Internal and External Flows, Volume 1, The Fundamentals of Computational Fluid Dynamics. Butterworth-Heinemann. ELSEVIER, $2^{\mathrm{a}}$ edição.

Hodge, W. (1952). The Theory and Applications of Harmonic Integrals. Cambridge University Press.

Jiang, G. e Shu, C.-W. (1996). Efficient implementation of weighted ENO schemes. J. Comput. Phys., $126: 206-228$.

Jiang, G.-S. e Wu, C. C. (1999). A high-order WENO finite difference scheme for the equations of ideal magnetohydrodynamics. J. Comput. Phys., 150:561-594.

Kamm, J., Brock, J., Brandon, S., Cotrell, D., Jhonson, B., Knupp, P., Rider, W., Trucano, T. G., e Weirs, V. G. (2008). Enhanced Verification Test Suite for Physics Simulation Codes. Relatório técnico, Los Alamos National Laboratory.

Karabasov, S. A. e Golovizin, V. M. (2012). Direct numerical simulations of compressible vortex flow problems. Advanced Fluid Dynamics, páginas 1-38.

Kemm, F. (2010). A comparative study of TVD-limiters-well known limiters and an introduction of new ones. Int. J. Numer. Methods Fluids, 67:404-440.

Kim, M. e Leer, W. (2003). A new VOF-based numerical scheme for the simulation of fluid flow with free surface. part I: New free surface-tracking algorithm and its verification. Int. J. Numer. Methods Fluids, 42:765-790.

Kuan, K. B. e Lin, C. A. (2000). Adaptive quick-based scheme to approximate transport. AIAA, $38: 2233-2237$.

Kun, X. (2002). A well-balanced gas-kinetic scheme for the shallow-water equations with source terms. J. Comput. Phys., 178(2):533-562.

Kurokawa, F. A. (2009). Um esquema 'upwind' para leis de conservação e sua aplicação na simulação de escoamentos incompressiveis $2 D$ e $3 D$ laminares e turbulentos com superfícies livres. Tese de Doutorado, Instituto de Ciências Matemáticas e de Computação. Universidade de São Paulo.

Landau, L. D. e Lifschitz, E. M. (1980). Fluid Mechanics 2nd. Ed. Pergamon Press, Oxford. 
Launder, B. e Spalding, D. (1974). The numerical computations of turbulent flows. Int. J. Numer. Methods Fluids, 15:127-146.

Lax, P. e Liu, X.-D. (1998). Solution of two dimensional Riemann problems of gas dynamics by positive schemes. SIAM J. Sci. Comput., 19:319-340.

Lax, P. e Wendroff, B. (1960). System of conservation laws. Commun. Pur. Appl. Math., 13:217-237.

Le, S. (2011). Re-examining the quickest algorithm for two-dimensional incompressible fluids. Int. J. Numer. Methods Fluids, 67(11):1470-1499.

Leonard, B. (1988a). Simple high-accuracy program for convective modeling of discontinuities. Int. J. Numer. Methods Fluids, 8:1291-1318.

Leonard, B. P. (1979). A stable and accurate convective modelling procedure based on quadratic upstream interpolation. Comput. Methods. Appl. Mech. Engrg., 19(1):59-98.

Leonard, B. P. (1988b). Universal limiter for transient interpolation modeling of the advective transport equations: The ULTIMATE conservative difference scheme. Technical Memorandum TM-100916-88-11, NASA, 1988.

LeVeque, R. (1992). Numerical Methods for Conservation Laws. Birkhauser Verlag.

LeVeque, R. (1997). Wave Propagation Algorithms for Multidimensional Hyperbolic Systems. $J$. Comput. Phys., 131:327-353.

LeVeque, R. J. (2004). Finite-volume methods for hyperbolic problems. Cambridge University Press.

LeVeque, R. J. (2012). Clawpack. http://depts.washington.edu/clawpack/.

Lima, G. A. B., Ferreira, V. G., Cirilo, E. R., Castelo, A., Candezano, M. A. C., Tasso, I. V. M., Sano, D. M. C., e Scalvi, L. V. A. (2012). A continuously differentiable upwinding scheme for the simulation of fluid flow problems. Appl. Math. Comput., 218:8614-8633.

Lin, C. H. e Chieng, C. C. (1991). Characteristic-based flux limiters of an essentially third-order flux-splitting method for hyperbolic conservation laws. Int. J. Numer. Methods Fluids, 13:287-307.

Lin, C.-H. e Lin, C. A. (1997). Simple high-order bounded convection scheme to model discontinuities. AIAA, 13:563-565.

Liu, X.-D. e Lax, P. D. (1996). Positive schemes for solving multi-dimensional hyperbolic systems of conservation laws. J. Comput. Fluid Dyn., 5:133-156.

Mahadevan, I., Ryu, W. S., e Samuel, A. D. T. (1998). Fluid 'rope trick' investigated. Nature, 392:140.

Majmudar, T., Varagnat, M., Hartt, W., e McKinley, G. (2010). Nonlinear dynamics of coiling in viscoelastic jets (preprint). arXiv/1012.2135. 
Martin, J. C. e Moyce, W. J. (1952). An experimental study of the collapse of liquid columns on a rigid horizontal plane. Philos. Trans. Roy. Soc. London Ser. A, 244:312-324.

McComb, W. (1990). The physics of fluid turbulence. Oxford University Press.

McKee, S., Tomé, M. F., Ferreira, V. G., Cuminato, J. A., Castelo, A., Souza, F. S., e Mangiavacchi, N. (2008). The MAC method. Comput. Fluids, 37:907-930.

McLeish, T. e Larson, R. (1998). Molecular constitutive equations for a class branched polymers: The Pom-Pom polymer. J. Rheol., 42:101-110.

MFiX, N. E. T. L. (2011). Multiphase flow with interphase exchanges. http://mfix.netl.doe.gov.

Morrison, J. F. (2007). The interaction between inner and outer regions of turbulent wall-bounded flow. Phil. Trans. R. Soc. A, 365:683-698.

Movagar, K., Reza, M., Fariborz, R., e Goharpey (2010). Efect of elasticity parameter on viscoelastic fluid in pipe flow using Extended Pom-Pom Model. Iran. J. Chem. Chem. Eng., 29-3:83-94.

Murrone, A. e Guillard, H. (2008). Behaviour of upwind scheme in their low Mach number limit: III. Preconditioned dissipation for a five equation two phase model. Comput. Fluids, 37:1209-1224.

Nikitin, K. D., Olshanskii, M. A., Terekhov, K. M., e Vassilevski, Y. V. (2011). A numerical method for the simulation of free surface flows of viscoplastic fluid in 3d. J. Comput. Math., 29:605-622.

Nóbrega, J. M., Carneiro, O. S., Pinho, F. T., Paulo, G. S., Tomé, M. F., Castelo, A., e Cuminato, J. A. (2007). The phenomenon of jet buckling: experimental results and numerical predictions. In The Polymer Processing Society 23rd Annual Meeting.

Nóvais, A. e de C. Cunha, M. C. (2003). Notas em Matemática Aplicada, 4. Métodos Numéricos para Equações Diferenciais Parciais. SBMAC.

Oishi, C. M., Martins, F. P., e, M. F. T., Cuminato, J. A., e McKee, S. (2011). Numerical solution of the extended Pom-Pom model for viscoelastic free surface flows. J. Non-Newton. Fluid, 166:165-179.

Oishi, C. M., Martins, F. P., Tomé, M. F., e Alves, M. A. (2012). Numerical simulation of drop impact and jet buckling problems using the extended Pom-Pom model. J. Non-Newtonian Fluid Mech., 169-170(0):91-103.

Orszag, S. e Tang, C.-M. (1979). Small-scale struture of two-dimensional magnetohydrodynamic turbulence. J. Fluid Mech., 90:129-143.

Pelanti, M. (2005). Wave Propagation Algorithms for Multicomponent Compressible Flows with Applications to Volcanic Jets. Tese de Doutorado, University of Washington.

Platzman, G. W. (1964). An exact integral of complete spectral equations for unsteady one-dimensional flow. Tellus, 16(4):422-431.

Pope, S. (2010). Turbulent Flows. Cambridge University Press. 
Qiu, J. e Shu, C.-W. (2003). Finite difference WENO schemes with Lax-Wendroff-type time discretizations. SIAM J. Sci. Comput., 24:2185-2198.

Qiu, J.-M. e Shu, C.-W. (2011). Conservative high order semi-lagrangian finite difference WENO methods for advection in incompressible flow. Journal of Computational Physics, 230(4):863-889.

Queiroz, R. e Ferreira, V. (2010). Development and testing of high-resolution upwind schemes. Upwind schemes for incompressible free surface flows. VDM Verlag Dr. Muller Aktiengesellschaft \& Co. KG.

Raad, P. E. e Bidoae, R. (2005). The three-dimensional eulerian-lagrangian marker and micro cell method for the simulation of free surface flows. J. Comput. Phys., 203(2):668-699.

Rai, A., Dandapat, B. S., e Poria, S. (2008). Circular hydraulic jump in generalized-newtonian fluids. arXiv/0809.2231.

Ritter, A. (1982). Die fortpflanzung der wasserwellen. Z. Ver. deut. Ing., 36.

Roache, P. J. (1994). Perspective: A Method for Uniform Reporting of Grid Refinement Studies. F. Fluid. Eng. - T. ASME, 116(3):405-413.

Roe, P. L. (1981). Approximate Riemann solvers, parameter vectors, and difference schemes. $J$. Comput. Phys., 43:357-372.

Roe, P. L. (1985). Some contributions to the modelling of discontinuing flows. Lectures in Applied Mathematics, 22:163-192.

Roe, P. L. (1986). Characteristic-based schemes for the Euler equations. Ann. Rev. Fluid. Mech., $18: 337-365$.

Roe, P. L. e Baines, M. J. (1982). Algorithms for advection and shock problems. In Proceeding of the Fourth GAMM Conference on Numerical Methods in Fluid Mechanics. Braunchweig:Vieweg.

Samtaney, R. e Pullin, D. I. (1996). On initial-value and self-similar solutions of the compressible Euler equations. Phys. Fluids, 8(10):2650-2655.

Santos, F. L. P., Ferreira, V. G., Tomé, M. F., Castelo, A., Mangiavacchi, N., e McKee, S. (2012). A marker-and-cell approach to free surface 2-d multiphase flows. Int. J. Numer. Methods Fluids, páginas $\mathrm{n} / \mathrm{a}-\mathrm{n} / \mathrm{a}$.

Sharp, D. (1984). An overview of Rayleigh-Taylor instability. Physica D, 12:3-18.

Shi, J., Zhang, Y.-T., e Shu, C.-W. (2003). Resolution of high order WENO schemes for complicated flow structures. J. Comput. Phys., 186:690-696.

Shih, T.-H., Liou, W. W., Shabbir, A., Yang, Z., e Zhu, J. (1995). A new k- $\epsilon$ eddy viscosity model for high reynolds number turbulent flows. Comput. Fluids, 24(3):227-238.

Shu, C.-W. (2006). Numerical Methods for Hyperbolic Conservation Laws. Relatório técnico, Brown University. 
Shu, C.-W. e Osher, S. (1989). Efficient implementation of essentially non-oscillatory shock-capturing schemes, II. J. Comput. Phys., 83:32-78.

Simões, A. L., Candezano, M. A. C., Schulz, H. E., Porto, R. M., e Ferreira, V. G. (2011). Comparacão de esquemas numéricos para as equacões de Saint-Venant usando codigos livres. In XIX Simpósio Brasileiro de Recursos Hídricos. Maceió, AL. Brazil.

Sod, G. A. (1978). A survey of several finite difference methods for systems of nonlinear hyperbolic ocnservations laws. J. Comput. Phys., 27:1-31.

Sondak, D. L. e Pletcher, R. H. (1995). Applications of wall functions to generalized nonorthogonal curvilinear coordinate systems. AIAA Journal, 33:33-41.

Song, B., Liu, G. R., Lam, K. Y., e Amano, R. S. (2000). On a higher-order bounded discretization scheme. Int. J. Numer. Methods Fluids, 32:881-897.

Spalding, D. B. (1972). A novel finite difference formulation for differential expressions involving both first and second derivatives. Int. J. Numer. Meth. Eng., 4:551-559.

Spekreijse, S. (1986). Multigrid Solution of the steady Euler equations. Tese de Doutorado, Delft University of Technology.

Stecca, G., Siviglia, A., e Toro, E. (2010). Upwind-biased FORCE schemes with applications to free-surface shallow flows. J. Comput. Phys., 229:6362-6380.

Stoker, J. J. (1956). Water waves: the mathematical theory with applications. Interscience Publishers, New York.

Sweby, P. e Baines, M. (1981). Convergence of Roe's scheme for the general non-linear scalar wave equation. Numerical Analysis Report, 8:81.

Sweby, P. K. (1984). High-resolution schemes using flux limiters for hyperbolic conservation laws. SIAM J. Numer. Anal., 21:995-1011.

Tao, W. Q. (2000). Recent Advances in Computational Heat Transfer. Science Press, Beijing.

Tao, W. Q., He, Y. L., Li, Z. Y., e Qu, Z. G. (2005). Some recent advances in finite volume approach and their applications in the study of heat transfer enhancement. Int. J. Therm. Sci., 44:623-643.

Teng, Z. H. (2010). Exact boundary conditions for the initial value problem of convex conservation laws. J. Comput. Phys., 229:3792-3801.

Tennekes, H. e Lumley, J. L. (1972). A First Course in Turbulence. The MIT Press.

The Center for Astrophysical Thermonuclear Flashes (2001). ASCI Flash Center, University of Chicago. http://flash.uchicago.edu/flashcode/doc/FLASH1.6/. 
Tomé, M. F., Castelo, A., Afonso, A. M., Alves, M. A., e Pinho, F. T. (2012). Application of the log-conformation tensor to three-dimensional time-dependent free surface flows. Journal of Non-Newtonian Fluid Mechanics, 175-176(0):44-54.

Tomé, M. F., Castelo, A., Ferreira, V. G., e MacKee, S. (2008). A finite difference technique for solving the Oldroyd-B model for 3D-unsteady free surface flows. J. Non-Newton. Fluid, 154:179-206.

Tomé, M. F., Mangiavacchi, N., Castelo, A., Cuminato, J. A., e McKee, S. (2001). GENSMAC 3D:A numerical method for solving unsteady three-dimensional free surface flows. Int. J. Numer. Methods Fluids, 37:747-796.

Toro, E. F. (1999). Riemann Solvers and Numerical Methods for Fluid Dynamics. A Practical Introduction. 2nd. Edition. Springer-Verlag.

Toro, E. F. e Titarev, V. A. (2006). Derivative Riemann solvers for systems of conservation laws and ADER methods. J. Comput. Phys., 212:150-165.

van Driest, E. R. (1956). On turbulence flow near a wall. Journal of Aeronautical Sciences, $37: 1007-1011$.

van Leer, B. (1973). Towards the ultimate conservative difference scheme I: The quest of monotonicity. Lectures Notes in Physics, 18:163-168.

van Leer, B. (1974). Towards the ultimate conservative difference scheme II. Monotonicity and conservation combined in a second-order scheme. J. Comput. Phys., 14:361-370.

van Leer, B. (1977). Towards the ultimate conservative difference scheme III. Upstream-centered finite-difference schemes for ideal compressible flow. J. Comput. Phys., 23:263-275.

van Leer, B. (1979). Towards the ultimate conservative difference scheme V. A second order sequel to Godunov's method. J. Comput. Phys., 32:101-136.

Verbeeten, W. (2001). Computational polymer melt rheology. Tese de Doutorado, Technische Universiteit Eindhoven.

Verbeeten, W. M. H., Peters, G. W. M., e Baaijens, F. P. (2002). Viscoelastic analysis of complex polymer melt flows using the eXtended Pom Pom model. J. Non-Newt. Fluid Mech., 108:301-326.

VonNeumann, J. e Richtmyer, R. D. (1950). A method for the numerical calculation of hydrodynamic shocks. J. Appl. Phys., 21:232-237.

Wan, Z.-H., Zhou, L., e Sun, D.-J. (2012). Robustness of the hybrid DRP-WENO scheme for shock flow computations. Int. J. Numer. Methods Fluids, 70(8):985-1003.

Wang, W. e Wang, M. (2011). Application of $\kappa-\varepsilon$ model on the numerical simulation of a semi-confined slot turbulent impinging jet. In 2011 Fourth International Joint Conference on Computational Sciences and Optimization. Kunming $\mathscr{E}$ Lijiang, Yunnan, China. 
Warming, R. F. e Beam, R. W. (1976). Upwind second order difference schemes and applications in aerodynamic flows. AIAA Journal, 24:1241-1249.

Waterson, N. P. e Deconinck, H. (2007). Design principles for bounded higher-order convection schemes - a unified approach. J. Comput. Phys., 224:182-207.

Watson, E. (1964). The radial spread of a liquid jet over a horizontal plane. J. Fluid Mech., 20III:481-499.

Wei, J.-J., Yu, B., Tao, W.-Q., e Kawaguchi, Y. (2006). A new general convective boundedness criterion. Numer. Heat Transfer. B., 49:585-598.

White, F. M. (2006). Viscous fluid flow. McGraw-Hill.

Wilcox, D. (1994). Turbulence Modeling for CFD. D.C.W. Industries Inc.

Woodward, P. e Colella, P. (1984). The numerical simulation of two-dimensional fluids flow with strong shocks. J. Comput. Phys., 54:115-173.

wxMaxima (2011). http://andrejv.github.com/wxmaxima/.

Xing, Y. e Shu, C.-W. (2005). High order finite difference WENO schemes with the exact conservation property for the shallow water equations. J. Comput. Phys., 168:206-227.

Xue, S. C., Phan-Thien, N., e Tanner, R. I. (2002). Upwinding with deferred correction (UPDC): an effective implementation of higher-order convection schemes for implicit finite volume methods. $J$. Non-Newtonian Fluid Mech., 108:1-24.

Yakhot, V. e Orszag, S. A. (1986). Renormalization group analysis of turbulence I. Basic theory. SIAM J. Sci. Comput., 51:1-3.

Yu, B., Tao, W. Q., Zhang, D. S., e Wang, Q. W. (2001). Discussion on Numerical Stability and Boundedness of Convective Discretized Scheme. Numer. Heat Transfer. B., 40:343-365.

Zahran, Y. H. (2008). Central ADER schemes for hyperbolic conservation laws. J. Math. Anal. Appl., 346:120-140.

Zalesak, S. (1987). A preliminary comparison of modern shock-capturing schemes: linear advection. In Advances in Computer Methods for Partial Differential Equation VI. Vichnevetsky R. Stepleman RS(eds) IMACS: New Brunswick, NJ.

Zijlema, M. (1996). On the construction of a third-order accurate monotone convection scheme with application to turbulent flows in general domains. Int. J. Numer. Methods Fluids, 22:619-641. 